\title{
Refining the Definition of Cultural Levels at Karabi Tamchin: A Quantitative Approach to Vertical Intra-Site Spatial Analysis
}

\section{By}

\section{Kirsten L. Anderson}

\author{
A Thesis \\ Submitted to the Faculty of Graduate Studies \\ In Partial Fulfilment of the Requirements \\ For the Degree of
}

\section{Master of Arts}

Department of Anthropology

University of Manitoba

Winnipeg, Manitoba

(C) June 2003 
THE UNIVERSITY OF MANITOBA

FACULTY OF GRADUATE STUDIES

COPYRIGHT PERMISSION PAGE

Refining the Definition of Cultural Levels at Karabi Tamchin:

A Quantitative Approach to Vertical

Intra-Site Spatial Analysis

BY

Kirsten L. Anderson

A Thesis/Practicum submitted to the Faculty of Graduate Studies of The University of Manitoba in partial fulfillment of the requirements of the degree

of

Master of Arts

Kirsten L. Anderson $@ 2003$

Permission has been granted to the Library of The University of Manitoba to lend or sell copies of this thesis/practicum, to the National Library of Canada to microfilm this thesis and to lend or sell copies of the film, and to University Microfilm Inc. to publish an abstract of this thesis/practicum.

The author reserves other publication rights, and neither this thesis/practicum nor extensive extracts from it may be printed or otherwise reproduced without the author's written permission. 


\section{- Abstract -}

The visual identification of archaeological levels can be difficult when the stratigraphy is complex. In this study, a Geographic Information System (GIS) is applied to a three-dimensional intra-site spatial analysis of artefact and bone distributions from Karabi Tamchin, a Middle Palaeolithic site from the Crimea, Ukraine. The distribution of archaeological materials is examined using the $\mathrm{K}$-means statistical clustering method, combined with a series of data transformations to identify and interpret the vertical and horizontal spatial organization of the site. Results indicate that K-means cluster analysis, used in conjunction with GIS, provides a means of testing the integrity of archaeological levels as well as identifying palimpsests. Within levels, K-means is used to identify discrete clusters of archaeological materials. Through an analysis of cluster contents, it is possible to reconstruct and compare patterns of spatial organization on the site, contributing to current debates regarding the cognitive complexity of Neanderthal populations. 


\section{- Acknowledgements -}

I would like to thank Dr. Alexander Yevtushenko, who directed the excavation of Karabi Tamchin and gave me permission to work with the three-dimensional data and Dr. Victor Chabai of the Institute of Archaeology, Ukrainian Academy of Sciences. Prof. Reid Ferring, University of North Texas, provided valuable comment on the geoarchaeology of the site. Excavations of Karabi Tamchin in 1999 were made possible by a grant from the Deutsche Forschungsbereicht for the project "Funktionale Variabilität des Späten Mittelpaläolithikums der Halbinsel Krim, Ukraine". Initial radiocarbon dates for levels $\mathrm{II}$ and IV were also obtained with funding from the DFG. Excavations in 2000 and 2001 and further radiocarbon dating was made possible by a grant from the Social Sciences and Humanities Research Council of Canada.

I would also like to thank the members of my committee for their time, knowledge and assistance. My thesis advisor, Dr. Ariane Burke provided me with the opportunity to study a Middle Palaeolithic rock shelter in the Crimea, Ukraine, which was an incredible and unforgettable experience. Dr. Persis Clarkson and Dr. Mary Benbow took time to read carefully through the manuscript, providing valuable advice and improvements for the final draft.

Thanks are also due to David Mosscrop, Paul Cooley and Dr. David Ebert for their helpful advice and patience regarding the often-painful attempt to wrap my head around GIS. Their expertise and encouragement helped me to develop this thesis beyond what I thought was possible, while improving my knowledge and interest in the use of GIS for archaeological research.

Throughout the research and writing of this thesis, many people have been a constant source of support and were often called upon to rescue me from "the cave." I wish to dedicate this thesis to them, as they each have had an incredible impact on my life. In particular, I would like to thank my mother and father for always being there to tell me that I could do it, even when I assured them I couldn't. I also want to thank my sister "Reege," who was always around to make me egg sandwiches and my dear friends Sabrina, Misty, Leslie and Ryan whose humour and mutual caffeine addiction kept me motivated. Finally, I would like to thank my boyfriend Darryl, who has been there through thick and thin and probably knows as much about spatial analysis as I do! Without his love, patience and an uncanny ability to rationalize everything, I would have never made it through to the end. For that, I am eternally grateful. 


\section{- Table of Contents -}

Abstract

$\begin{array}{ll}\text { Acknowledgements } & \text { ii }\end{array}$

Table of Contents

List of Figures vii

List of Tables viii

Chapter One: Introduction

1.1 Introduction 1

1.2 Spatial Analysis of Middle Palaeolithic Sites 2

1.3 Three-Dimensional Spatial Analysis 4

1.3.1 K-means Cluster Analysis 5

1.3.2 Geographical Information Systems in Archaeology 6

$\begin{array}{ll}1.4 \text { Thesis Organization } & 8\end{array}$

\section{Chapter Two: Spatial Analysis in Archaeology}

2.1 Introduction

2.2 What is Spatial Analysis? 11

2.2.1 Intra-Site Spatial Analysis 13

2.2.2 Three-Dimensional Spatial Analysis $\quad 14$

2.3 Palaeolithic Spatial Organization 16

2.3.1 Factors of Disturbance $\quad 19$

2.4 Methods of Spatial Analysis $\quad 22$

2.4.1 Dimensional Analysis of Variance 24

2.4.2 Nearest Neighbour Analysis $\quad 27$

2.4.3 K-Means Cluster Analysis 32

$\begin{array}{ll}2.5 \text { Conclusions } & 37\end{array}$

Chapter Three: Geographical Information Systems and Archaeology

3.1 Introduction $\quad 39$

3.2 What is a GIS? 40

3.3 Vector and Raster Based GIS $\quad 41$

3.4 Advantages for Archaeological Applications 44

3.5 Potential Limitations $\quad 48$

3.5.1 Costs and User Knowledge 49

3.5.2 Quality of Data Analysis 51

3.6 Three-Dimensional GIS Applications 53

3.7 Future Developments 55 


\section{Chapter Four: Introduction to the Dataset}

4.1 Introduction $\quad 57$

4.2 The Crimea

$\begin{array}{ll}4.3 \text { Karabi Tamchin } & 61\end{array}$

4.4 Site Stratigraphy 64

4.5 The Dataset $\quad 67$

4.6 Potential Problems to be Addressed 68

4.7 Conclusions $\quad 69$

\section{Chapter Five: Methods}

5.1 Introduction $\quad 71$

5.2 Tests of Randomness

5.3 Re-Sampling Strategy $\quad 72$

5.4 GIS and Three-Dimensional Visualization $\quad 76$

5.5 K-Means Cluster Analysis $\quad 77$

5.5.1 SPSS v. $10 \quad 78$

5.5.2 Kintigh's Tools for Quantitative Archaeology $\quad 79$

5.6 Data Preparation 80

5.6.1 Mapmaker Pro and ArcView 3.2 80

5.6.2 K-Means Analysis Using Kintigh's Tools $\quad 84$

for Quantitative Archaeology

5.6.2.1 Z Expansion Factors $\quad 85$

5.6.2.2 Data Standardization Trial $\quad 85$

5.6.2.3 Combined Z Expansion Factor and $\quad 86$

Data Standardization Trial

5.7 Significance Testing $\quad 88$

5.8 Conclusions $\quad 91$

\section{Chapter Six: Results}

6.1 Introduction 93

6.2 K-means Cluster Analysis SPSS v.10

6.3 Kintigh's Tools for Quantitative Archaeology 96

Level by Level Analysis

6.3.1 Level II/2 97

6.3.1.1 Significance Testing $\quad 99$

6.3.2 Level III

100

6.3.2.1 Significance Testing 103

6.3.3 Level IV 104

$\begin{array}{ll}\text { 6.3.3.1 Significance Testing } & 106\end{array}$

6.4 Conclusions

106 


\section{Chapter Seven: Discussion and Interpretation}

7.1 Introduction

7.2 SPSS v.10 Analysis

7.2.1 SPSS v.10 K-means Cluster 1

7.2.2 SPSS v.10 K-means Cluster 2

112

7.2.3 SPSS v.10 K-means Cluster 3

112

7.2.4 SPSS v.10 K-means Cluster 4

113

7.2.5 Conclusions

114

7.3 Kintigh's K-means Cluster Analysis 114

7.3.1 Level $\Pi 1 / 2$

114

7.3.1.1 Level II/2: 3-Cluster Solution $\quad 115$

$\begin{array}{ll}\text { 7.3.1.2 Significance } & 117\end{array}$

7.3.1.3 Level II/2: 8-Cluster Solution $\quad 118$

7.3.1.4 Significance $\quad 123$

$\begin{array}{ll}\text { 7.3.2 Level III } & 125\end{array}$

7.3.2.1 Level III: 4-Cluster Solution $\quad 126$

$\begin{array}{ll}\text { 7.3.2.2 Significance } & 128\end{array}$

7.3.2.3 Level III: 6-Cluster Solution $\quad 130$

7.3.2.4 Significance $\quad 133$

$\begin{array}{ll}\text { 7.3.3 Level IV } & 136\end{array}$

7.3.3.1 Level IV: 3-Cluster Solution $\quad 137$

$\begin{array}{ll}\text { 7.3.3.2 Significance } & 139\end{array}$

7.3.3.3 Level IV: 5-Cluster Solution $\quad 141$

7.3.3.4 Significance $\quad 143$

7.4 Conclusions

145

\section{Chapter Eight: Conclusions}

8.1 Introduction 149

$\begin{array}{ll}\text { 8.2 Spatial Organization at Karabi Tamchin } & 149\end{array}$

8.3 Three-Dimensional Spatial Analysis 150

8.4 K-means and Geographical Information Systems 152

8.5 Contributions to Archaeology 153

8.6 Future Recommendations 155

\section{Appendix A - Frequency Distribution of Total Faunal Materials Recovered from Karabi Tamchin}

\section{Appendix B - Horizontal Artefact Distributions} Re-Sampled Levels 
Appendix C - K-Means Cluster Solutions Kintigh's Tools for Quantitative Archaeology

Appendix D - Chi-Square Significance Results

Appendix E - Horizontal K-Means Cluster Distributions Re-Sampled Levels

Appendix F - Three-Dimensional K-Means Cluster Distributions Re-Sampled Levels

Bibliography 


\section{- List of Figures -}

4.1 Map of Crimean Peninsula Including the Site of Karabi Tamchin

4.2 Stratigraphic Profile of the Cultural/Natural Strata from Karabi Tamchin:

West Wall Profile

5.1 Total Three-Dimensional Artefact Distribution for Karabi Tamchin

74

5.2 Excavated Grid of $1 \mathrm{~m} \mathrm{x} 1 \mathrm{~m}$ Square Units at Karabi Tamchin $25 \mathrm{~m}^{2}$

81

5.3 X and Y Coordinate Transformations for Archaeological Grid $25 \mathrm{~m}^{2}$

82

5.4a Archaeological Shapefile Level II Horizontal Distribution

83

5.4b Archaeological Shapefile Level III Horizontal Distribution

83

5.4c Archaeological Shapefile Level IV Horizontal Distribution

84

5.4d Archaeological Shapefile Level V/2 Horizontal Distribution 84

6.1: Histogram Plot of Z-Value Distribution for All Levels: Arrows represent potential clusters in the dataset

94

6.2a Final Clustering Results for the 4-Cluster K-means Analysis: Cluster 1996

6.2b Final Clustering Results for the 4-Cluster K-means Analysis: Cluster 296

6.2c Final Clustering Results for the 4-Cluster K-means Analysis: Cluster $3 \quad 96$

6.2d Final Clustering Results for the 4-Cluster K-means Analysis: Cluster $4 \quad 96$

6.3 Sum Squared Error Graph for Level II/2 98

6.4 Sum Squared Error Graph for Level III 101

6.5 Sum Squared Error Graph for Level IV 105

7.1 Three-Dimensional Distribution Level III - Vertical Overlap Between

Clusters 6-4 (highlighted in yellow) and 6-6 (highlighted in red)

135 


\section{- List of Tables -}

4.1 Corresponding Archaeological and Lithological Levels at Karabi Tamchin

4.2a Total Artefact Frequency and Percentage Distribution for Level II/2 and III

4.2b Total Artefact Frequency and Percentage Distribution for Level IV and V/2

5.1 Calculated Artefact Frequency Ratios and Sampling Percentages for Levels II/2, III and IV

5.2 Coordinate Scale Ranges ( $\min$ to max depth below datum) with Applied Z Expansion Factors

6.1 Z-Value Range for each Cluster in the SPSS v.10 K-Means Cluster Analysis

7.1 Z-Value Range for the Archaeologically Defined Levels and SPSS Clusters

7.2 Cluster 3-1 Artefact Frequency and Percentage Distribution for the Z-Expansion Factor-10 3 Cluster Solution, Level II/2

7.3 Cluster 3-2 Artefact Frequency and Percentage Distribution for the Z-Expansion Factor-10 3 Cluster Solution, Level II/2

7.4 Cluster 3-3 Artefact Frequency and Percentage Distribution for the Z-Expansion Factor-10 3 Cluster Solution, Level II/2

7.5 Cluster 8-1 Artefact Frequency and Percentage Distribution for the Z-Expansion Factor-10 8 Cluster Solution, Level II/2

7.6 Cluster 8-2 Artefact Frequency and Percentage Distribution for the Z-Expansion Factor-10 8 Cluster Solution, Level II/2

7.7 Cluster 8-3 Artefact Frequency and Percentage Distribution for the Z-Expansion Factor-10 8 Cluster Solution, Level II/2

7.8 Cluster 8-4 Artefact Frequency and Percentage Distribution for the Z-Expansion Factor-10 8 Cluster Solution, Level II/2

7.9 Cluster 8-5 Artefact Frequency and Percentage Distribution for the Z-Expansion Factor-10 8 Cluster Solution, Level II/2

7.10 Cluster 8-6 Artefact Frequency and Percentage Distribution for the Z-Expansion Factor-10 8 Cluster Solution, Level II/2

7.11 Cluster 8-7 Artefact Frequency and Percentage Distribution for the Z-Expansion Factor-10 8 Cluster Solution, Level II/2

7.12 Cluster 8-8 Artefact Frequency and Percentage Distribution for the Z-Expansion Factor-10 8 Cluster Solution, Level II/2

7.13 Cluster 4-1 Artefact Frequency and Percentage Distribution for the Z-Expansion Factor-10 4 Cluster Solution, Level III

7.14 Cluster 4-2 Artefact Frequency and Percentage Distribution for the Z-Expansion Factor-10 4 Cluster Solution, Level III

7.15 Cluster 4-3 Artefact Frequency and Percentage Distribution for the Z-Expansion Factor-10 4 Cluster Solution, Level III

7.16 Cluster 4-4 Artefact Frequency and Percentage Distribution for the Z-Expansion Factor-10 4 Cluster Solution, Level III 
7.17 Cluster 6-1 Artefact Frequency and Percentage Distribution for the Z-Expansion Factor-10 6 Cluster Solution, Level III

7.18 Cluster 6-2 Artefact Frequency and Percentage Distribution for the Z-Expansion Factor-10 6 Cluster Solution, Level III

131

7.19 Cluster 6-3 Artefact Frequency and Percentage Distribution for the Z-Expansion Factor-10 6 Cluster Solution, Level III

7.20 Cluster 6-4 Artefact Frequency and Percentage Distribution for the Z-Expansion Factor-10 6 Cluster Solution, Level III

132

7.21 Cluster 6-5 Artefact Frequency and Percentage Distribution for the Z-Expansion Factor-10 6 Cluster Solution, Level III

7.22 Cluster 6-6 Artefact Frequency and Percentage Distribution for the Z-Expansion Factor-10 6 Cluster Solution, Level III

7.23 Cluster 3-1 Artefact Frequency and Percentage Distribution for the Z-Expansion Factor-10 3 Cluster Solution, Level IV

138

7.24 Cluster 3-2 Artefact Frequency and Percentage Distribution for the Z-Expansion Factor-10 3 Cluster Solution, Level IV

7.25 Cluster 3-3 Artefact Frequency and Percentage Distribution for the Z-Expansion Factor-10 3 Cluster Solution, Level IV

139

7.26 Cluster 5-1 Artefact Frequency and Percentage Distribution for the Z-Expansion Factor-10 5 Cluster Solution, Level IV

7.27 Cluster 5-2 Artefact Frequency and Percentage Distribution for the Z-Expansion Factor-10 5 Cluster Solution, Level IV

7.28 Cluster 5-3 Artefact Frequency and Percentage Distribution for the Z-Expansion Factor-10 5 Cluster Solution, Level IV

142

7.29 Cluster 5-4 Artefact Frequency and Percentage Distribution for the Z-Expansion Factor-10 5 Cluster Solution, Level IV

7.30 Cluster 5-5 Artefact Frequency and Percentage Distribution for the Z-Expansion Factor-10 5 Cluster Solution, Level IV 


\section{- Chapter One - Introduction}

\section{1 - Introduction}

The application of spatial analysis to Palaeolithic sites can provide valuable information about the location of and relationship between prehistoric activities that took place within them. Spatial analysis considers activities at many different levels, including the association of artefacts, physical features, environments and their interactions (Clarke 1979: 460). Therefore, studies of spatial patterning have the potential to provide important information regarding the behaviour of past populations, creating a more complete understanding, beyond the functional analysis of artefacts. The goal of this thesis is to develop an appropriate method for the analysis and interpretation of threedimensional spatial organization within a Middle Palaeolithic rock shelter site. Comparisons will be made between visually defined cultural layers in a stratigraphic profile and the results of an objective, quantitative analysis using three-dimensional data. Once cultural levels have been defined, it may be possible to identify individual occupations within levels, thus providing an effective method for the analysis of archaeological palimpsests. The combined use of K-means cluster analysis and Geographic Information Systems will be applied to a three-dimensional dataset of archaeological materials for the interpretation of differential use of space (horizontal distribution) and the nature of occupational levels (vertical distribution) within the shelter.

The study region used for this research is the eastern section of the Crimean peninsula, in the Ukraine. This region was selected for analysis based on the availability of a three-dimensional dataset suitable for horizontal and vertical spatial analysis, as well 
as the unique opportunity to investigate spatial patterning within a Middle Palaeolithic rock shelter site in a region of archaeological significance. Investigations within the Crimean peninsula have identified numerous Middle Palaeolithic cave and rock shelter sites throughout the Crimean Mountain Ranges, however Karabi Tamchin has been the first stratified site identified within the highland region of the first mountain range (Yevtushenko et al. In Press a). Therefore, this site and the region in general, have the potential to answer many unanswered questions regarding Middle Palaeolithic settlement patterns and the behavioural characteristics of Neanderthal populations.

Within this proposed research, the following questions will be addressed:

1. Is it possible to develop a suitable method for quantitative three-dimensional spatial analysis, where stratigraphy is complex?

2. Can the horizontal analysis of artefact and bone clusters be used to interpret differential spatial organization?

3. Is it possible to identify unique occupational characteristics such as the presence of archaeological palimpsests through vertical analysis?

4. Can the results of this spatial analysis be used to interpret behavioural characteristics, thus contributing to the current debate concerning the organization of space within Neanderthal populations?

5. To what degree does the application of GIS improve the analysis of three-dimensional spatial patterning in archaeology?

\section{2 - Spatial Analysis of Middle Palaeolithic Sites}

Spatial analysis within archaeology is based on the premise that the location and relationship between various artefact clusters (assuming un-disturbed) are a reflection of past activities (Simek 1984a: 405). This evidence offers insight into the behavioural organization of space within a prehistoric context. Within Palaeolithic archaeology, the analysis of artefact distributions generally focuses on the analysis of cave and rock shelter sites, where evidence of cooking, tool manufacture, hearth locations and sleeping 
areas have been identified by the distribution faunal and lithic materials (Simek 1984a: 406). Unfortunately, Palaeolithic sites are rarely found in an undisturbed context; therefore, the relationships and significance between artefact distributions can be difficult to distinguish.

The analysis of spatial patterning within archaeological distributions must allow for two important considerations (Gregg et al. 1991: 152). First, the characteristics of the distribution must be identified based on the spatial structure of the deposit. Specifically, it is necessary to determine whether materials are clustered or evenly distributed. Once this has been established, it is necessary to evaluate the presence or absence of artefact clustering, as this can provide the important information regarding behavioural organization and site disturbances. Natural processes such as taphonomic, chemical, biological and physical factors (both depositional and post-depositional) can have tremendous influence over the spatial characteristics of a distribution. Therefore, the presence of these processes should be identified and incorporated into any evaluation of human behavioural activities (Simek 1984a: 406; Gregg et al. 1991: 152).

Recent interpretations of Middle Palaeolithic spatial distributions have suggested that the use of space among Neanderthal populations does not demonstrate a complex pattern of organization. Rather, variability within the distributions has been compared to the patterning of non-human carnivores (Pettitt 1997) and interpreted as very basic, pragmatic responses to the environment, which can be distinguished from the complexity of modern human behaviour (Binford 1996; Mellars 1996). Others (Vaquero 1999; Vaquero and Pasto 2001) have criticized this interpretation based on evidence for structured use of space in some Middle Palaeolithic sites. While these interpretations 
offer plausible explanations for the spatial distribution of some Middle Palaeolithic sites, it is equally important to consider the potential problems associated with depositional and post-depositional disturbances, which can obscure natural patterning.

\section{3 - Three-Dimensional Spatial Analysis}

Archaeological investigations of spatial patterning have focused on the horizontal distribution of materials in a two-dimensional setting. Horizontal, intra-site spatial analysis generally involves the analysis of a single, defined cultural level (Whallon 1973, 1974; Simek 1984b; Hietala 1984; Carr 1987). The results are used to interpret the organization of space within a particular site, based on concentrations of various artefacts throughout the defined level. While these analyses can reveal important information concerning social and behavioural organization, they cannot consider the vertical distribution of materials and therefore must rely on adequate definition of archaeological levels. Within Palaeolithic archaeology, the consideration of horizontal and vertical stratigraphy is extremely important, as sites are often palimpsests of materials, or accumulations of individual occupations appearing as one single occupation. In these situations, the interpretation of spatial organization and behaviour patterning is dependent upon the differentiation of individual deposits (Spikins et al. 2002: 1236). Therefore, it seems logical to develop a method of spatial analysis that can incorporate three dimensions of variability.

Within an archaeological context, stratigraphy may be clearly defined, facilitating the identification of distinct, cultural deposits. However, when the stratigraphy is complex, it may be difficult to identify individual levels and to determine the true 
relationship between artefacts. Spikins et al. (2002: 1236) suggest that the threedimensional analysis of artefact distributions can potentially improve the interpretation of site stratigraphy, especially when it is difficult to distinguish individual levels. While horizontal intra-site spatial analysis has produced successful results, investigations of vertical distributions have been neglected, despite obvious benefits. Therefore future applications of spatial analysis within archaeology need to develop an appropriate methodology for three-dimensional analysis in situations where the identification of individual cultural layers is not visibly distinct.

\subsection{1 - K-means Cluster Analysis}

The $\mathrm{K}$-means cluster analysis is a quantitative approach that classifies points into a specified number of clusters according to their distance from a cluster centre, or cluster mean. This method of cluster analysis has been applied in the horizontal investigation of archaeological distributions (Simek 1984a; Rigaud and Simek 1991; Gregg et al. 1991; Vaquero 1999); however, it also holds potential for three-dimensional analysis (Koetje 1989; Belcher and Paradis 1992). The program computes point assignments through a series of iterations, until reassigning points and re-computing cluster means (based on overall points in a cluster) produces no changes. Once completed, the most appropriate classifications are determined and the results are displayed (SPSS: 1999). K-means can be run using both two and three-dimensional coordinates. To run k-means in three dimensions, it is necessary to transform the third dimension in order to avoid equal variance assumptions (see chapter 2 for a detailed discussion). Several transformations can be easily applied to the $Z$ coordinate scale resulting in the creation of meaningful 
clusters in three-dimensional space. The available transformations and the results of their application will be discussed in detail within chapters 5 and 6 .

One of the benefits of the k-means cluster analysis is the option to save the cluster membership of each point in a dataset as a column in the original database. By saving the cluster membership, the database can be queried by cluster, making it possible to examine the attributes of points within each cluster, including the location and frequency of individual artefact types. One of the drawbacks of the program concerns the visualization of three-dimensional clusters, as the program can only display the horizontal distribution of clusters. While this is potentially limiting for an interpretation of the vertical distribution, the ability to save cluster memberships permits the researcher to export the results of the k-means cluster analysis. An alternative program, such as Geographical Information Systems (GIS), which offers superior graphic and analytical capabilities, can then be employed for the three-dimensional visualization and interpretation of clusters.

\subsection{2 - Geographical Information Systems in Archaeology}

Geographical Information Systems (GIS) have only recently been introduced in archaeology and have largely been used to visualize archaeological data. Despite limited application, GIS have become a valuable tool for analysis. Cultural resource managers and archaeologists have taken advantage of the flexibility and capability of GIS and as a result, the majority of analytical applications in archaeology have focused on predictive modelling (Green 1990: 5). Through predictive modelling, it is possible to predict the 
location of sites through the study of known sites, based on the premise that similar types of archaeological sites should be located in similar types of environments.

More recently, GIS has been applied to archaeological spatial analysis for both regional and intra-site studies. At the intra-site level, the application of GIS allows researchers to study individual settlement strata, with the inclusion of attribute data, such as environmental and cultural features (Csaki et al. 1995: 85). The ability to incorporate this information into any aspect of the analysis demonstrates considerable strength over traditional methods of pattern recognition. An additional benefit for the analysis of intrasite spatial patterning involves the three-dimensional graphical capabilities of GIS, which are well suited to the spatial properties of archaeological data.

While it will focus specifically on the Middle Palaeolithic, the results of this study should prove applicable to a variety of archaeological sites and to rock shelter sites in particular. Knowledge regarding the behaviour of prehistoric hunter-gatherer populations is based upon the interpretation of archaeological materials and their associations in space. Therefore, the improvement of analytical methods will lead to the development of more complete and meaningful investigations. This research will also allow for the evaluation of GIS as a potential three-dimensional tool for the analysis of archaeological materials, with particular focus on intra-site spatial analysis. The comparison of quantified and visually assessed cultural layers will demonstrate the effectiveness of using statistical clustering methods in situations where stratigraphy is complex and visual identifications are difficult. Potentially, it will also demonstrate the limitations of both visual and quantitative methods in spatial archaeology. 
Most importantly, this research aims to develop an innovative method of analysis for the interpretation of palimpsest levels, which are present in the majority of Palaeolithic deposits. Given the difficulty in dealing with palimpsests, this research will provide a greater understanding of site formation processes as well as an assessment of the utility of vertical cluster analysis. The identification of within-level palimpsests will contribute to a better understanding of the types of activities and occupations at Karabi Tamchin.

\section{4 - Thesis Organization}

This thesis will begin with a detailed discussion of the development of spatial analysis within archaeology, including an investigation of the potential utility of Geographic Information Systems for archaeological investigations. Chapter two will provide an overview of spatial studies within archaeology, with particular focus on Palaeolithic applications. Following this discussion, a series of spatial analytical methods are evaluated. K-means cluster analysis emerges as the most appropriate method for the present dataset.

Within chapter three, Geographic Information Systems (GIS) are introduced, with a detailed description of the concept of GIS, including the potential advantages and disadvantages associated with archaeological applications. This chapter will also provide an evaluation of the use of GIS for three-dimensional spatial analysis. It is determined that current GIS are limited for analysis in three dimensions; however, their graphical capabilities can be combined with quantitative methods for the interpretation of spatial distributions. 
Chapter four provides a description of the dataset. The Middle Palaeolithic rock shelter site of Karabi Tamchin (Crimea, Ukraine) is introduced, outlining the characteristics of the dataset and the importance of this site to the development of regional Palaeolithic settlement patterns. The chapter concludes with an outline of the potential problems to be addressed in the analysis.

The methodological framework for this thesis is presented in chapter five. Specifically, the development of an appropriate three-dimensional digital dataset is discussed, followed by an outline of the methods employed for three-dimensional cluster analysis and the statistical evaluation of cluster contents. The results of these methods are presented in chapter six and are used to identify significant clustering solutions for further interpretation.

Chapter seven provides an analysis and interpretation of the results from chapter six. The spatial patterning of statistically significant cluster solutions is evaluated for the identification of horizontal artefact distributions as a possible indication of the differential use of space at Karabi Tamchin. In addition, the vertical distribution of clusters is examined for the identification of intra-level palimpsests. These interpretations are then discussed in relation to Palaeolithic archaeology, with specific focus on the characteristics of Neanderthal occupations.

Chapter eight concludes with an evaluation of the methods employed in this thesis, with particular focus on their suitability for three-dimensional spatial analysis. The potential limitations associated with this research are discussed, followed by recommendations for the improvement of current and future spatial studies within archaeology. 


\section{- Chapter Two - Spatial Analysis in Archaeology}

\section{1 - Introduction}

Today, everyday activities are associated with specific living areas, generating a blueprint of the way individuals function within a defined space. Similar patterns can be observed in the distributions of archaeological materials within a defined occupational space, allowing researchers to identify particular patterns in the behavioural characteristics of prehistoric populations. These activities are structured by important factors, including socio-economic composition of the group occupying the site, length and intensity of occupation and the relationship of tasks performed at the site to the subsistence system (Binford 1977: 9; Ferring 1984: 118). Therefore, the analysis and interpretation of artefact distributions is a vital part of any archaeological investigation (particularly in prehistoric settings), as this information provides important evidence about behaviour.

While the importance of spatial analysis is appreciated in archaeology, in the context of this study it is necessary to develop a clear understanding of the methods available, with a careful consideration of their advantages and limitations. It is also necessary to select a method of analysis that is well suited to the characteristics of the site and artefact distribution under investigation. Far too often in the past, the data have been forced to conform to the method. This chapter will provide a discussion of spatial analysis tools within archaeology, including a detailed evaluation of potential methods. In consideration of the goals of this research and the characteristics of the available dataset, 
$\mathrm{K}$-means cluster analysis is chosen as an appropriate method for the three-dimensional analysis of artefact distributions at Karabi Tamchin.

\section{2 - What is Spatial Analysis?}

The importance of spatial information in archaeology is demonstrated by the results generated by the creation of detailed maps and plans, and the precision with which the three-dimensional coordinates of artefacts and features are recorded at an archaeological site (Clarke 1977: 6). Spatial archaeology can be defined as:

“...the retrieval of information from archaeological spatial relationships and the study of the spatial consequences of former hominid activity patterns within and between features and structures and their articulation within sites, site systems and their environment" (Clarke 1979: 460).

Through the analysis of the unique spatial patterning of materials, it is possible to identify and interpret behavioural activities within an archaeological context. This information can influence the development and understanding of prehistoric populations at various levels of resolution, from the study of individual sites to regional interpretation.

The initial development of spatial analysis within archaeology focused on the characterization of global patterning within archaeological sites, using such methods as nearest neighbour (Whallon 1973), variance/mean ratio (Dacey 1973) and dimensional analysis of variance (Whallon 1974). Other methods that have been developed in recent years, though not as widely used, include: ring and sector method (Stapart 1989; 1990), Hodder and Okell's A analysis (Hodder and Okell 1978), local density analysis (Johnson 1984; Graham 1980), factor analysis (Cowgill 1968), Fisher's exact test (Spurling and Hayden 1984), permutation procedure (Berry et al. 1984), Fourier methods (Carr 1987) and k-means cluster analysis (Kintigh and Ammerman 1982). Only dimensional analysis 
of variance, nearest neighbour and k-means will be discussed here, as they demonstrate potential applicability for the goals of this research. In recent years, there has been a general expansion of archaeological applications of spatial studies, which have demonstrated the importance of site formation processes and context, such as geological processes, site topography and non-human occupations (Rigaud and Simek 1991: 200). Evidence from ethnographic investigations has also been incorporated into spatial studies (Schiffer 1972; Binford 1978; Spurling and Hayden 1984); these studies suggest that individual activities are spatially distinct and therefore, tools used to perform specific activities should demonstrate unique spatial patterning (Rigaud and Simek 1991: 200).

Prior to the development and application of sophisticated statistical methodology, archaeologists relied on the visual interpretation of artefact distributions for the identification of spatial patterning (to a certain extent that is still true today). During excavation, archaeological layers are generally defined visually, in a manner that cannot always be replicated, as interpretation relies on a subjective visualization of the dataset based on the researcher's inclinations and intuitions (Kintigh and Ammerman 1982: 33; Simek and Larick 1983: 166; Koetje 1992: 177;). Limitations, including the time of day and the direction or intensity of sunlight, can affect the record (photography, drawing) of a stratigraphic profile, making accurate representation difficult. When the stratigraphy is complex and level boundaries are unclear, profiles should be regarded as subjective interpretations only. Moreover, visualization provides an effective tool for identifying obvious clusters of artefacts; however, statistics can indicate relationships that may not be apparent for a given dataset. 
A growing interest in spatial analysis in archaeology has lead to the development of quantitative methods for pattern recognition that offer an objective, statistically based scientific methodology (Whallon 1973: 266). Quantitative methods are more reliable and replicable than visual interpretation (Berry et al. 1980: 55; Koetje 1992: 177-8); they limit the effects of observer bias, as the analysis is based completely on relationships in the data. This is especially true in situations where large artefact distributions may be patterned in ways that are not readily identifiable to the naked eye.

\subsection{1 - Intra-Site Spatial Analysis}

Spatial patterning can be investigated at a regional (inter-site) or intra-site level of analysis, depending on the available data and the types of questions being asked. Intrasite spatial analysis operates on two levels: the inferential level, which concerns the reconstruction and explanation of behaviours and activities (which are non-observable), and the operational level, which derives relationships between archaeological materials (Carr 1984). The operational level of analysis consists of three steps. First, the distribution of artefacts is analysed to determine how they are arranged (whether they are randomly scattered, aggregated as clusters or spatially aligned). Once the distribution pattern has been defined, the spatial limits and areas of concentration are determined for each artefact type. The dataset is then used to evaluate whether different artefact types demonstrate similar arrangements throughout the site. Finally, significant relationships between artefacts are identified (Whallon 1973: 266-7; Carr 1984: 106-7). The inferential level of analysis allows the archaeologist to reconstruct different activities, their frequency and spatial organization. From these activities, information regarding 
seasonality, population and group composition, site function, kinship, social organization and many additional behavioural and environmental traits is inferred (Carr 1984: 106). It is through such reconstructions that archaeologists are able to develop an understanding of past behaviours, moving beyond the basic analysis of artefacts.

\subsection{2 - Three-Dimensional Spatial Analysis}

Operationally, spatial analysis has generally been restricted to the analysis of the horizontal distribution of artefacts within levels (Whallon 1973, 1974; Simek and Larick 1983; Simek 1984b; Ferring 1984; Keeley 1991; Stapart and Street 1997; Vaquero 1999). This is unfortunate, considering that most archaeological excavations include the painstaking recovery and recording of materials in three dimensions. The consideration of archaeological sites as occupying three dimensions is extremely important for three basic reasons. First, every archaeological site should be considered as a series of deposits laid down on a pre-existing topographic surface, which provides some level of vertical relief affecting the distribution of archaeological materials. Second, archaeological materials are usually deposited along with natural or anthropogenic (cultural) sediments, which can pattern their distribution. Sites will build up different layers of both cultural and sedimentary deposits over time, through an accretionary process. Finally, postdepositional processes can affect the distribution of archaeological materials, both horizontally and vertically (Koetje 1992: 177). The horizontal distribution of materials is important in terms of hominid spatial behaviour, however Vertical distributions should also be examined as they provide unique information regarding site formation processes as well as post-depositional disturbance. 
Palaeolithic sites that have been identified as palimpsest deposits, or accumulations of debris from multiple superimposed occupations, have often been avoided in spatial studies in favour of more pristine sites, due to the difficulties associated with identifying individual occupation phases (Villa 1982: 278; Koetje 1990: 257). While palimpsests are very difficult to interpret, three-dimensional spatial analysis can be applied to these sites to help identify distinct occupation phases.

Once cultural deposits are identified, it is possible to develop a relative chronostratigraphic sequence and to compare the units (Villa and Courtin 1983: 268). Traditionally, this is done by creating a stratigraphic profile. In some situations, separations between natural and cultural layers are distinct, facilitating the classification of materials. In contrast, relatively diffuse or un-patterned vertical deposits of materials also often require the definition of arbitrary levels. A more reliable, quantitative approach is required for the identification of cultural deposits based on both the horizontal and vertical distribution of cultural data. This would allow researchers to refine the definition of stratigraphic layers and identify vertical distinctions within poorly stratigraphically defined deposits.

Part of the problem regarding the application of spatial analytic techniques in archaeology is a frequent lack of fit between the sample and the method. In many situations, "...our methods have been allowed to dictate our questions rather than the other way around," (Kintigh 1990: 197). This can have a serious effect on the integrity of the results, throwing into question the validity of the resulting interpretations. Many of the spatial analytical methods used in archaeology were borrowed from other disciplines including mathematical ecology, economics, geography, architecture and ethnology 
(Clarke 1979: 453; Carr 1987: 236). Despite their apparent applicability, methods such as dimensional analysis of variance (Whallon 1973) and nearest neighbour analysis (Clark and Evans 1954; Whallon 1974) were applied to archaeological datasets without critical evaluation, resulting in methodological and theoretical problems, as underlying principles and statistical assumptions were generally inappropriate for archaeological data (Vierra and Taylor 1977: 317; Blankholm 1991: 40). Therefore, it is necessary to develop a clear understanding of the quantitative methods available, including their suitability in association with the available dataset and the intended purpose of the analysis.

\section{3 - Palaeolithic Spatial Organization}

Palaeolithic archaeology involves the excavation of open-air sites and cave or rock shelter sites. Erosional processes, including fluvial movement and drainage, or the deep burial of sites due to geomorphological processes, has led to the inevitable destruction or concealment of numerous open-air deposits (Straus 1979: 332). As a result, Palaeolithic research has come to depend on the excavation of cave and rock shelter sites, which are more easily located and offer conditions that are more amenable to preservation. Palaeolithic cave and rock shelter deposits have the potential to reveal behavioural information about the prehistoric hunting and gathering groups that occupied them. Despite their potential importance, quantitative spatial studies are rarely conducted in Palaeolithic archaeology, as few sites have been excavated under suitable conditions for the collection and recording of spatial data. As a result, these spatial studies have only made a limited contribution to our understanding of prehistoric spatial behaviour (Mellars 1996: 269; Galanidou 2000: 243; Vaquero and Pasto 2001: 1209). 
The investigation of spatial patterning within Middle Palaeolithic occupations has resulted in a debate as to the behaviour of Neanderthal populations (Mellars 1996; Binford 1996; Pettitt 1997; Vaquero 1999; Vaquero and Pasto 2001). One of the primary goals of Palaeolithic archaeology has been the identification and understanding of past behaviour, including differences between ancient hominids and modern humans. Suggested differences in the cognitive capabilities of Neanderthals have led some researchers to argue that lithic technology, symbolic expression and the organization of space should be relatively simple when compared to the complexity of Upper Palaeolithic occupations. Therefore, the analysis of the use of space is key to understanding these differences and appreciating the cognitive capabilities of Neanderthal populations (Vaquero 1999: 493; Vaquero and Pasto 2001: 1209).

Social organization among anatomically modern humans revealed through ethnoarchaeological evidence, is reflected in the spatial arrangement of living/working areas; an apparent absence of such organization within ancient hominid populations is used to suggest a more basic level of spatial behaviour (Binford 1996: 234). Pettitt (1997) also suggests that the spatial organization of cave and rock shelter sites occupied by hominids is very basic and can be compared to that of non-human carnivores. The occupation of cave and rock shelter sites by non-human carnivores creates a distribution of materials with higher concentrations of faunal debris in specific areas of the site. In addition, carnivores will use certain areas for defecation and carcass processing, while others will be reserved for sleeping (Pettitt 1997: 218). Pettitt compares these characteristics to current interpretations (such as Binford's) of Neanderthal spatial patterning, to suggest that the use of space by carnivores and hominids is quite similar: 
i.e., there is a segregation of living and processing/refuse areas, while animal processing and associated activities are carried out away from the sleeping area (Pettitt 1997: 218).

In an investigation of spatial patterning at the Middle Palaeolithic rock shelter of Abric Romani (Capellades, Spain), Vaquero and Pasto (2001) have demonstrated that hearth-related accumulations are similar to patterns identified in Upper Palaeolithic sites. This provides direct evidence that prehistoric hominids were organizing space, contrary to Binford's (1996) and Pettitt's (1997) interpretations. The spatial distribution of Middle Palaeolithic deposits “...change according to specific factors, such as group size, occupation length and kind of activity" (Vaquero 1999: 503); therefore, it is necessary to develop a complete understanding of spatial characteristics before it is possible to interpret behavioural patterning. Vaquero and Pasto (2001: 1219) argue that comparisons between hominid and non-human carnivore use of space do not consider the spatial significance of hearth-related assemblages, which demonstrate differential use of space unlike that observed in non-human carnivore occupations. The performance of activities around a hearth by hominids would likely result in closer similarities with modern human patterns (based on ethnoarchaeological evidence) than that of non-human carnivores. A comparison between the spatial organization of Neanderthal and non-human carnivores may be premature, as current interpretations of Neanderthal populations are based on a relatively limited collection of sites, with varying degrees of preservation and available spatial information.

Mellars (1996) has also suggested that the spatial patterning of Middle Palaeolithic sites demonstrates organization that reflects pragmatic responses to the environment rather than cognitive or social complexity. While there are patterns in the 
distribution of archaeological materials in Middle Palaeolithic sites, it is unclear whether they can be attributed to complex behavioural patterning. At a very basic level, spatial patterning could be interpreted as a natural response "...to the simple functional requirements and constraints involved in the organization of various economic, technological and social activities within specific, restricted areas" (Mellars 1996: 311). According to Mellars the location of activities in close proximity to a hearth would only demonstrate a desire to be warm or comfortable while working. Vaquero (1999: 503) argues that the very nature of human behaviour past and present is based on pragmatic responses and therefore, this is not an appropriate interpretation of Middle Palaeolithic spatial patterning. Neanderthals may have demonstrated a very basic level of spatial organization (Binford 1996; Pettitt 1997; Mellars 1996); however, adaptations to a constrained environment such as a cave or rock shelter setting would require alterations to the existing topography or differential organization of space (Mellars 1996: 269; Pettitt 1997: 219; Galanidou 2000: 270).

\subsection{1 - Factors of Disturbance}

The arguments discussed above provide contrasting interpretations of the meaning of spatial patterning in terms of hominid behaviour, however it is equally important to consider the nature of Middle Palaeolithic deposits and the Palaeolithic record in general. Middle Palaeolithic cave and rock shelters were used as both long-term occupation sites as well as seasonal and temporary shelters, depending on the context. From an archaeologist's perspective, differences in occupation lengths may be difficult to distinguish, as the restricted setting of a cave or rock shelter can inhibit the spatial 
differentiation of individual occupations (Straus 1979: 333). In a given amount of time, any number of individuals could have occupied a cave or rock shelter. Therefore, a large group could have occupied a site for a short period, or smaller groups could have occupied a site for a long period, resulting in similar artefact frequencies and distribution. Within archaeology, it is commonly understood that most Middle Palaeolithic sites can be reconstructed as palimpsests (Straus 1979: 334; Farizy 1994: 153; Mellars 1996: 269; Pettitt 1997: 219; Galanidou 2000: 243). These layers appear as one continuous occupation; however, they may contain any number of temporal or ephemeral occupations. Straus (1979: 334) suggests that they may be the result “ $\ldots$ of repeated occupations of the same spot either during a short period of time or during a period of very slow sedimentation." Only through careful, strategic excavation and analysis is it possible to accurately identify and interpret the depositional events associated with palimpsests. Given the presence of a palimpsest, it may not be possible to tease out the effects of context (topography of the site) from cultural behaviour or to interpret the nature of the occupation(s) (base camp, short-term occupation, kill site), as these may have differed over time, producing a mixed signature.

The interpretation of spatial patterning in an archaeological setting must also consider the possibility that the original deposition and characteristics of artefact distributions have been obscured by post-depositional factors including non-human scavenging (Marean and Bertino 1994) and down slope or vertical displacement (Rick 1976; Villa 1982; Rowlett and Robbins 1982; Villa and Courtin 1983). Kroll and Price (1991: 197) argue that it is necessary to avoid the simplistic assumption that spatial patterning is the direct result of human activities. Cave and rock shelter sites offer 
adequate habitation sites for non-human carnivores, such as hyena and cave bear. The presence of these animals can have an impact on the distribution of archaeological deposits, first by the consumption and displacement of faunal materials and secondly, through the accumulation of new materials, which is dependent on the length of occupation (Marean and Bertino: 1994: 751). This can result in a mixture of deposits from human and non-human activities. Marean and Bertino (1994: 751) argue that this can be especially problematic in situations where the cave or shelter was used as a seasonal habitation site, with periods of disuse. Human and non-human deposits cannot always be distinguished, however it is possible to identify the presence of carnivores on faunal material (gnawing, teeth and puncture marks), thereby providing evidence to assist spatial interpretations.

The vertical movement of artefacts due to depositional and post-depositional disturbances can obscure spatial patterns, further confusing level distinctions. Archaeological sites will often suffer the effects of animal burrowing, tree root damage, periods of erosion, the displacement of soil resulting from digging or levelling and the effects of trampling (Villa and Courtin 1983: 271-72). These events can occur at differential rates, with varying degrees of disturbance.

Within Palaeolithic occupations, the accumulation of artefacts on a living floor provides the only existing cultural evidence for the use of space, as there is a general absence of structural remains (Rick 1976: 133). In periods of abandonment, natural sediments from the exfoliation of roof and wall materials will accumulate on the living floor as large boulders and gravel, called eboulis (Rowlett and Robbins 1982: 73; Koetje 1993: 115). Sterile accumulations of eboulis may separate occupational levels, resulting 
in a sequence of well-stratified layers. While these types of deposit provide clear, distinct stratigraphic profiles, cultural levels lying superimposed between layers of eboulis still need to be distinguished from one another. This is not always possible when postdepositional processes have resulted in the vertical or horizontal movement of artefacts, even when there is no visible evidence of disturbance within the stratigraphic profile (Villa 1982: 278). Villa and Courtin (1983) have also studied the effects of vertical displacement resulting from trampling within cave sites. The results of this experiment have demonstrated that normal activities within a site could result in the admixture of individual deposits, especially in situations where the accumulation of sediments occurs at a very slow rate (Speth and Johnson 1976: 47-8; Villa and Courtin 1983: 279). Therefore, continuous occupation by hominids or non-human carnivores could potentially result in greater levels of vertical and/or horizontal displacement and disturbance within cave and rock shelter sites.

\section{4 - Methods of Spatial Analysis}

The 1970s marked the beginning of a period of assessment and advancement of spatial analysis in archaeology, leading to the theoretical and methodological development of techniques first borrowed from ecology in the 1950s. The first methods applied to spatial analysis in archaeological investigations included the Dimensional Analysis of Variance (Whallon 1973) and Nearest Neighbour Analysis (Whallon 1974), (discussed in detail, below). Both of these methods are based on quantitative tests involving data counts or coordinate data (Djindjian 1988: 96; Blankholm 1991: 40). The evaluation and development of spatial analysis developed in two major directions. The 
first involved the evaluation of formation processes and the archaeological pattern, while the second was concerned with the continued growth of new and more appropriate methodologies (Blankholm 1991: 41). Implicit assumptions in models such as Dimensional Analysis of Variance and Nearest Neighbour defined activity areas as relatively uniform in their density, shape, size and composition regardless of the type of site, or cultural affiliation (Blankholm 1991: 41). This is obviously an inappropriate assumption for archaeological investigations, given the known degree of variability between and within individual sites.

In recent years, traditional methods of spatial analysis have been modified through methodological refinement, enabling archaeologists to use methods that are suitable for the investigation of a specific problem or type of site (Whallon 1973; Speth and Johnson 1976; Hietala and Stevens 1977; Pinder et al. 1979). For example, the kmeans cluster analysis provides a middle ground between the subjectivity of visual interpretation and the inappropriate and often limiting assumptions associated with quantitative methods (Ammerman et al. 1983: 211). The procedure is relatively well understood and accessible (see discussion below). K-means analysis also relieves some of the difficulties identified with traditional quantitative analysis, including the boundary problems associated with nearest neighbour analysis and the definition and placement of an appropriate grid for quadrat approaches such as the dimensional analysis of variance (Kintigh and Ammerman 1982: 61). Subsequent improvements in the application and utility of methods have also resulted in a variety of methods available for spatial analysis within archaeology depending on the requirements of the researcher (see Hodder and Orton 1976, 1982; Carr 1984; Kintigh 1990; and Wandsnider 1996 for a full discussion). 
The selection of an appropriate method for intra-site spatial analysis must consider the advantages and disadvantages associated with the method, as well as the nature of dataset or site under investigation. This research is aimed at examining spatial patterning of artefacts within four archaeologically defined levels at Karabi Tamchin as well as verifying the integrity of levels identified using traditional methods in the field. The location and artefact contents of clusters identified within each level will be examined horizontally for the identification of potential activity areas. Clusters will also be examined vertically in three dimensions for the interpretation of site formation processes, including the possible identification of within-level palimpsests. The selection of an appropriate method must consider these requirements, while providing an analysis that is replicable and capable of adequately analyzing artefact distributions in three dimensions. Furthermore, the method must identify clusters within the distribution, including the individual artefact memberships (including the type of artefact) and location of artefacts within each cluster distribution. While this research focuses on the analysis of a Middle Palaeolithic rock shelter site, the selected method of analysis must demonstrate utility across a wide range of archaeological contexts. This is imperative, as the identification of an appropriate method of three-dimensional analysis in archaeology is one of the primary goals of this research.

\subsection{1 - Dimensional Analysis of Variance}

Grid-based methods compare raw frequency counts from individual squares with the expected frequency distribution of a random sample of data, to determine how the sample compares with a random distribution (Orton 1982: 8). It does not require the user 
to identify the point provenience for each artefact in the distribution, providing a general analysis of artefact concentrations. One of the main benefits of grid-based or quadrat methods is that archaeological information is often collected in a similar fashion. Excavations may recover the relative counts of archaeological materials per unit without identifying the exact three-dimensional location of each artefact (Hodder and Orton 1976: 36). In these situations, a quadrat analysis will provide a suitable analysis for the available dataset; however in situations where the exact coordinates are known, a more complex locational analysis may be more appropriate.

The Poisson method is a quadrat method that can be used to determine the relative spatial characteristics of a distribution. It is based on the understanding that the placement of a grid of equal quadrats over a random distribution of objects will generate a grid cell count, which approximates a Poisson distribution for the expected distribution (Hodder and Orton 1976: 33-34; Carr 1984: 140). As a result, it is possible to utilize the ratio of variance to the mean to determine the relative spatial characteristics of the sample. A Poisson, or random distribution has a variance: mean ratio of 1 , while a clustered distribution will generate a value greater than 1 and an aligned distribution will be lower than 1 (Hodder and Orton 1976: 34). Therefore, it is possible to compare the observed frequency of a given sample with the expected frequency to determine relative spatial characteristics.

The Dimensional Analysis of Variance (DAV) is another more common gridbased method. Whallon (1973) originally introduced the DAV method to archaeology however, applications have generally been limited to investigations designed to test the technique (Carr 1984: 145). This method eliminates some of the problems associated 
with the Poisson method; it works by assessing the distribution of archaeological materials through the application of various grid systems of different size and shape, to identify which grid system displays the most significant artefact clustering. Whatever grid is selected becomes a representation of a meaningful distribution, as "...counts of artefacts within its grid cells are taken to approximate counts of artefacts within behaviourally significant clusters, rather than within arbitrarily size grid units," (Carr 1984: 145).

While the application of various grid sizes determines the most appropriate arrangement for a given dataset, the use of grid generally influences the area of the distribution considered in the analysis. Within the DAV method, a grid must represent a square or rectangular shape (where length can only be twice as long as the width) (Whallon 1973: 267). This obviously places considerable constraints on the dataset, especially in an archaeological context, where sites may have been deposited in naturally confined areas such as caves or rock shelters, creating a unique distribution that may not be adequately represented by a square or rectangle. Whallon (1973:268) suggests that the use of "dummy units" can fill in empty grid cells, or an entire distribution can be analyzed as distinct sub-sections. Each of these procedures can minimize the problems associated with a square or rectangular grid; however, they can also influence the results of the analysis. Artefact clusters are generally obscured by the use of square units and the analysis of sub-sections will require the researcher to determine meaningful subsections for investigation, which may crosscut artefact distributions, or may further obscure spatial patterning, which is not visibly distinct. 
While the DAV method places restrictions on the size and shape of datasets that can be analyzed, it is replicable and allows researchers to identify clustering within the distributions, including the location and artefact memberships of individual clusters as defined by the grid (Whallon 1973: 268). Unfortunately, this method is not suitable for sites with unique spatial constraints, such as Palaeolithic cave and rock shelter sites. The use of arbitrary grid cells, or the subdivision of materials into distributions that fit into a square or rectangle shaped analysis, can minimize or completely erase spatial evidence associated with this type of setting.

This thesis examines the spatial distribution of artefacts within a spatially confined setting. Therefore, the use of a square or rectangular grid is not an appropriate method of analysis based on the arguments presented above. The DAV method does not require the use of point provenience information as it relies completely on the frequency counts of artefacts within each grid square. This provides the most convincing evidence that a grid-count method is not suitable for the intended goals of this research, as a third dimension is irrelevant within this method of analysis.

\subsection{2 - Nearest Neighbour Analysis}

Nearest neighbour analysis was originally developed for the analysis of population distributions in ecology (Clark and Evans 1954) however, this type of analysis has also been used in archaeology for the study patterning within distributions of archaeological materials (Whallon 1974; Pinder et al. 1979; McNutt 1981; Durand and Pippin 1992). Nearest neighbour analysis is designed to interpret spatial relationships between distributions of objects (i.e., artefacts) to create an overall description of the 
spatial characteristics of the sample. This procedure is identified as a method of point pattern analysis, which is defined as "...those, which give information of the scale and structure of non-randomness or association in spatial distributions" (Graham 1980: 107). The method interprets the distribution based on the calculated distance between points to determine whether the distribution is random (equal distance between points) or concentrated (clustered groups of points).

The nearest neighbour statistic "...is derived from the observation that given a random distribution of points in space, the expected mean nearest distance is a function of density" (Durand and Pippin 1992: 264). Specifically, the statistic is a ratio of the mean distances for all points and their nearest neighbour, divided by the mean distance that would be representative of a random distribution of the same sample (Graham 1980: 107; Kintigh 1990: 167). This coefficient is used to determine the degree of variability between a random sample and the actual distribution. If the sample represents a random distribution, where points are independent of one another, the resulting value will be close to 1.0. A clustered (non-random) distribution will result in values less than 1.0. Finally, if the sample is evenly distributed and does not demonstrate a random or clustered distribution, the nearest neighbour value will be greater than 1.0 (Graham 1980: 108; Kintigh 1990: 167).

Nearest neighbour analysis offers an easy, quantitative method for characterizing a distribution of points; however, there are several problems associated with the procedure. Most importantly, applications of the nearest neighbour method have identified a boundary effect (Pinder et al. 1979; Whallon 1974; McNutt 1981; Kintigh 1990). Within an archaeological context, the exact boundary of the site may not be 
known. Therefore defining a suitable area for the analysis can have considerable influence on the resulting nearest neighbour coefficients. Kintigh (1990: 168) argues that the boundary of a site may include the entire excavated area, or it may be defined by the distribution of materials, excluding those areas from which no materials were recovered. While both solutions seem like logical boundary definitions, the resulting nearest neighbour coefficients are substantively different. Carr (1984: 157) refers to this difficulty as a framing problem, where the size of the area under consideration can influence the resulting spatial interpretations.

An additional boundary problem concerns the nature of the observed dataset versus the expected dataset. Within the observed dataset, nearest neighbour averages are calculated within a defined study region, while any points located outside of the region are removed from analysis. As a result, the distance of points on either side of the boundary are not considered in the analysis. Therefore, a nearest neighbour distance will be calculated between points within the study region, regardless whether points on the edges are at a greater distance away (Pinder et al. 1979: 431; Carr 1984: 157-158). This can affect the overall nearest neighbour coefficient, by increasing the average observed distance.

The definition of boundaries for the application of nearest neighbour is problematic; furthermore, researchers need to consider the cultural and temporal context when dealing with archaeological data. Nearest neighbour uses the location of individual points within a distribution to identify spatial patterns. Unfortunately, nearest neighbour analyzes the data independently of contextual information, such as the environmental setting, culture or types of materials under investigation (Kintigh and Ammerman 1982: 
32). Contextual information is the means archaeologists have to develop a foundation for the interpretation of behaviour based on spatial patterning.

Nearest neighbour analysis only provides a very basic interpretation of the spatial patterning within a distribution, which requires further examination to provide a meaningful analysis of complex spatial relationships within the data (Pinder et al. 1979: 443; Carr 1984: 160). While nearest neighbour analysis is used to establish an overall interpretation of spatial patterning within a particular study area, it cannot be used to examine differential degrees of clustering. The distribution of a specific artefact type may exhibit significant clustering in one section of the site, with a more random distribution throughout the rest of the site; however, the nearest neighbour analysis will average the distribution. Carr (1984: 160) argues that this method is less informative for intra-site spatial analysis than other available methods as it is only evaluates the relative density of artefact clustering and does not identify specific clusters within the dataset.

Archaeologists have the additional problem of establishing the temporal relationships in their data. Within point pattern analyses, a distribution of archaeological materials will be interpreted as a contemporary deposit and cannot consider potential differences in temporal space (Orton 1982: 2). In order to apply nearest neighbour analysis, Pinder et al. (1979: 442) argue that researchers need to determine “...the degree of precision or contemporaneity..." within the chosen dataset to ensure that artefacts are part of the same depositional event. Unfortunately, it can be difficult to establish whether a group of archaeological materials represents a contemporaneous deposit, especially when occupation periods are difficult to distinguish and the stratigraphic profile is complex. 
Several attempts to improve the accuracy and utility of nearest neighbour analysis have developed methods to correct the boundary problem. Hodder and Orton (1976: 4143) have attempted to minimize the problems associated with points located near the boundary by adding a series of randomly distributed points with equal density around the study area. In order to ensure accuracy, this method must be performed several times to develop an average nearest neighbour coefficient for the distribution. Pinder et al. (1979) have also attempted to improve nearest neighbour analysis through a modification of the original formula, which specifically defines the boundary as a square. This places heavy constraints on the dataset, especially when the distribution is better represented as a different shape (Carr 1984: 159). As a result, the benefits of minimizing the boundary effect may be offset by the introduction of new constraints to the dataset. Kintigh (1990: 170) suggest that attempts to minimize the boundary problem are often unsatisfactory and may require considerable modification to the dataset in order to improve the analysis.

When the advantages and disadvantages of the nearest neighbour method are compared with the intended goals of this research, it is clear that this type of analysis is not capable of identifying meaningful clusters of artefacts in archaeologically defined levels. While nearest neighbour could be used as a replicable method for the identification of global spatial patterning, this research is focused on the three dimensional analysis of artefact clusters and the interpretation of contents in terms of behaviour. In addition, excavations of Karabi Tamchin did not include the entire boundary of the original rock shelter, as the front of the shelter has eroded over time. The eastern part of the site also extends further, however this area was not excavated in order 
to leave a witness profile. Therefore defining a meaningful boundary for the identification of spatial patterning would be extremely difficult.

\subsection{3 - K-Means Cluster Analysis}

The increased sophistication of computer technology has provided archaeologists with "the means to define patterns of distribution, either those obvious to the human eye or those discerned by a computer using sophisticated quantitative, analytical techniques," (Kroll and Price 1991: 301). As a result, current methods are more accurate, efficient and powerful than traditional methods, with the capability to handle larger, more complex datasets. The greater application of these methods to archaeological situations and ethnoarchaeological verification (Kintigh and Ammerman 1982; Spurling and Hayden 1984; Carr 1987) has confirmed their utility.

The k-means analytical technique provides a simple method of cluster analysis, used when a sample is considered non-homogeneous (non-random). The procedure has been applied in a number of archaeological situations (Simek 1984b; Riguad and Simek 1991; Gregg et al. 1991; Koetje 1992; Enloe et el. 1994; Vaquero 1999) and is one of the most common clustering techniques used in archaeology. K-means is defined as a heuristic spatial analytical method, which involves a search for patterns in the distribution of data while at the same time attempting to discover the underlying principles of organization creating the patterns. These methods allow researchers to use their knowledge to interpret quantitative results in a meaningful way, using contextual characteristics of their distribution (Ammerman et al. 1983: 211; Simek et al. 1985: 229). 
The k-means cluster analysis is a non-hierarchical statistical method that attempts to minimize variance within clusters, while maximizing the distance between clusters. One of the main benefits of a non-hierarchical method is the ability to identify distinct patterns at a number of different scales. In addition, the application of k-means cluster analysis has demonstrated an ability to identify visually distinct spatial patterning while providing additional quantitative information when obvious patterning cannot be defined (Kintigh and Ammerman 1982: 38). Despite the obvious advantages of k-means, early applications tended to create ideal shape and size constraints on the clusters that are formed. Koetje (1990: 259) states that this is a major disadvantage of non-hierarchical methods; however, transformations can be applied to the dataset to minimize the effects of the imposed constraints (see chapter five).

The k-means programme classifies points into a specified number of clusters according to their distance from a cluster centre, or cluster mean, determined by the mean $\mathrm{X}$ and $\mathrm{Y}$ coordinates for all of the points in the sample (Simek and Larick 1983: 168, Ammerman et al. 1983: 212). The program computes point assignments through a series of iterations, until reassigning point and re-computing cluster means (based on overall points in a cluster) produces no changes. At this point, the most appropriate classifications are determined and the results are displayed. This programme assigns points to clusters that will minimize the Sum of the Squared Error (SSE), which is defined as "... the sum of the squared distances between all points in the distribution and their nearest centroid at a given clustering level" (Simek and Larick 1983: 169, Koetje 1990: 259). Once the analysis is complete, the clustering solution (number of clusters) with the smallest SSE value is considered as the most appropriate for the given sample. 
The SSE is expressed as a percentage of the maximum for each clustering solution and can be plotted against the number of clusters. The resulting plot is used by the analyst to identify the best number of cluster configurations (Hodson 1970: 311; Simek and Larick 1983: 169, Ammerman et al. 1983: 212). Inflection points along the plot indicate lower SSE values and represent better clustering solutions for the sample, making it possible to identify fine-scale patterning that may not be identified visually. Unlike quantitative methods such as the Dimensional Analysis of Variance and Nearest Neighbour (discussed above), the results of k-means analysis are associated with the original dataset; consequently it is possible to generate a detailed description of individual clusters, including the cluster memberships of each point within the distribution (Hodson 1970: 318). This allows the researcher to analyze individual clusters to determine potential relationships between clusters in a given solution, through the investigation of individual artefact concentrations and the spatial distribution of artefact types. Kintigh and Ammerman (1982: 43) suggest that researchers have the opportunity of characterizing each cluster "... according to their constituents, the number of points, the radius of the cluster, and any linear trend in the point distribution." Therefore, $\mathrm{k}$ means offers substantively greater possibilities regarding the interpretation of quantitative spatial patterning combined with contextual archaeological information.

While archaeological applications of $\mathrm{k}$-means have produced interesting results for horizontal distributions, few have considered the importance of a third dimension. The k-means technique is defined as an equal variance method searching for clusters with equal variance in all dimensions. The resulting analysis attempts to produce ideal spherical clusters. This is not always appropriate when considering archaeological levels 
and spatial distribution (Koetje 1992: 178). The inclusion of a third dimension allows researchers to investigate unique patterning and relationships between levels as well as within them that is not possible with two-dimensional applications (Belcher and Paradis 1992: 108; Koetje 1994: 161).

The analysis of three-dimensional distributions presents a unique problem for equal variance procedures. Within archaeology, the distribution of the vertical dimension is generally much smaller than the horizontal ( $\mathrm{X}$ and $\mathrm{Y}$ ) distributions. Without consideration of distribution differences, clusters will be formed with unequal treatment of all dimensions and greater weight will be assigned to the axis with the greatest variation (Koetje 1991: 189). Normal spherical clusters may not accurately reflect spatial distributions on archaeological sites, however. When three dimensions are considered, a "lenticular, lozenge or highly stretched ovate shape is easily the most common, idealized model of an archaeological level" (Koetje 1990: 259). The solution is the imposition of arbitrary dimensional space that avoids equal variance assumptions. This requires a conversion of the original, three-dimensional dataset into an appropriate dataset with equal analytical space in all dimensions.

An object's original coordinates $(\mathrm{X}, \mathrm{Y}$ and $\mathrm{Z})$ can be replaced on each axis with a $\mathrm{Z}$-score value. A $\mathrm{Z}$-score value is defined as "...the distance (expressed in standard deviations) between an observation within a set and the mean of the set," (Koetje 1991: 189). Through this process, an entirely new set of coordinates is created for each observation (artefact) in the sample. The use of the Z-score successfully transforms the entire distribution into a standard space, where the coordinate means for each axis are represented as zero (Koetje 1992: 179; Koetje 1993: 118). When the k-means analysis is 
performed, these transformations will affect the resulting clusters, producing results that are appropriate and meaningful.

An additional transformation for the improvement of distributional differences between vertical and horizontal dimensions is a $\mathrm{Z}$-axis expansion factor. This transformation places more weight on the Z-axis and can improve the ability to discriminate between various layers when the vertical distribution is small (Koetje 1990: 267; Koetje 1991: 189). Once the appropriate transformations have been conducted, it is possible to run the $\mathrm{k}$-means program under the assumptions of an equal variance procedure. The ability to tailor k-means software for archaeological analysis gives it an important advantage over other spatial analyses such as DAV and nearest neighbour analysis. Through the equalization of variance, the program can form clusters that are not heavily influenced by one dimension over another (Koetje 1990: 261; McKelway et al. 1992: 150). Once clusters are identified using k-means, individual objects can be entered into a GIS programme using their original coordinates to examine the true distribution of objects assigned to each cluster (McKelway et al. 1992: 150).

The development of GIS has provided innovative approaches for archaeological investigations, including the analysis of spatial distributions. The concepts of space and time are crucial to the understanding of archaeological site formation processes and the association of artefacts. One of the main benefits of GIS is that they are designed to utilize spatial data and is well suited to the requirements of space and time analysis. Many important areas of archaeological research (cultural resource management, regional and intra-site spatial analysis) can be supported by this technology (Lock and Harris 1996: 216) in ways that are not possible with conventional database programs. 
Unfortunately, current GIS demonstrate limited functional capability for quantitative analysis (see chapter 3 ) and are better suited to graphical display. It is possible however, to combine the results of the k-means analysis with GIS software to produce comprehensive results for three-dimensional intra-site spatial analysis.

\section{5 - Conclusions}

The importance of spatial patterning in archaeological research has resulted in the use of quantitative, analytical methods. The information thus provided can potentially provide evidence for the behavioural characteristics of hominid populations, their cognitive organization and the function of space. Unfortunately, cave and rock shelter sites can be subjected to a variety of depositional and post-depositional effects, creating a mixed assemblage that may not be easily interpreted. The problems associated with the excavation and interpretation of these sites can be resolved through careful development of the stratigraphic record as well as controlled excavation, including the recognition of horizontal distributions (Straus 1979: 337). In addition, the analysis of spatial patterning must consider the existing limitations and incorporate them into the interpretation. Most importantly, cave and rock shelter sites are often palimpsest deposits, where individual occupational episodes form one large assemblage.

Through the evaluation of current methods, k-means cluster analysis has been identified as the most suitable application for the goals of this research (see chapter five). $\mathrm{K}$-means offers a method that is replicable and reliable, with the flexibility to handle three-dimensional datasets. Through the analysis of three-dimensional cluster distributions combined with the graphical capabilities of GIS, it may be possible to identify the presence of palimpsest deposits, while interpreting the horizontal distribution 
of materials in terms of behavioural patterning within Karabi Tamchin. This research will provide an opportunity to evaluate the suitability of the k-means method and GIS for the analysis of Middle Palaeolithic occupations, while demonstrating the importance of three-dimensional analysis within archaeological investigations. 


\section{- Chapter Three - \\ Geographical Information Systems and Archaeology}

\section{1 - Introduction}

As outlined in the previous chapters, the intended goal of this thesis is to develop an effective and accurate method of interpreting the spatial distribution of archaeological materials in three dimensions. The development and application of spatial analysis has become a fundamental component in archaeological research at the regional and intra-site scales. Unfortunately, many studies are limited by the kinds of information that can be generated from the dataset. The recent introduction of Geographic Information Systems (GIS) in geography has effectively changed the role of spatial data and maps through the introduction of database analysis and cartographic display (Knoerl 1991: 107). Geographic Information Systems (GIS) are a powerful group of computer mapping programs, which are designed for the efficient storage, analysis and presentation of spatially referenced information. This chapter will discuss the concept of GIS, including the advantages and potential limitations associated with archaeological applications. A critical examination of potential applications suggests that archaeologists have only begun to exploit the full range of opportunities available for regional and intra-site analyses. In addition to functional limitations, the acceptance and integration of GIS has been restricted by high costs and a lack of sufficient education and training among researchers. The chapter will conclude with a detailed evaluation of the potential application of GIS for three-dimensional spatial analysis. Current GIS are limited in their ability to quantify three-dimensional datasets. However, it is possible to combine the 
graphical capabilities of GIS with an alternative statistical program to interpret the distribution of archaeological materials in three dimensions.

\section{2 - What are GIS?}

Computer technology has significantly improved the accuracy and efficiency of data collection and analysis. Geographic Information Systems (GIS) were first introduced in the late 1960s and have since presented archaeologists with an innovative and powerful research tool for the input, storage, manipulation and output of data (Marble 1990: 98; Fisher 1999: 5). While initial GIS studies were limited in function and application, continued development has greatly enhanced the utility of the software, rendering it suitable for use on a wide variety of research projects. Archaeologists generally define GIS as spatially referenced computer databases, which create a link between a graphically displayed point or feature (spatial data) and its record within a database (non-spatial/attribute data) (Maschner 1996b: 2; Wheatley and Gillings 2002: 60). A unique set of user-defined attributes is available for each spatial feature, allowing the researcher to analyze a particular dataset both visually and quantitatively. Attribute data can be defined as "...the information about the objects or phenomenon whose locations and spatial properties we have carefully recorded," most commonly available from inventories, fieldwork reports and record sheets (Wheatley and Gillings 2002: 60). For example, within archaeology, an artefact can be represented graphically as a point on a map, while information concerning its type, location, colour, size, etc., is stored in a database for reference and subsequent analysis. This definition of a Geographic Information System presents a very basic understanding of the technology. In addition to 
data storage, GIS programs are designed for the mathematical manipulation, analysis, capture, retrieval and display of spatially referenced real-world data (Kvamme 1989: 139; Burrough 1986: 6; Knoerl 1991: 99).

\section{3 - Vector-Based and Raster-Based GIS}

There are two principal types of GIS that can be employed, depending on the information available and the research questions to be answered; they are vector-based or raster-based. Each design is a reflection of the format used for the retrieval and storage of information, allowing the user to manipulate data in specific ways (Savage 1990: 23). Vector-based GIS use points, lines and polygons to represent discrete spatial data, such as artefact locations, roads or archaeological sites. In this respect, the images produced in a vector system are similar to the graphical representation of features in traditional maps (Kvamme and Kohler 1988: 503). Vector GIS are very efficient for data storage and are well suited to the management and evaluation of data distributed over large areas (Kvamme 1989: 154; Knoerl 1991: 99; Maschner 1996b: 3). Consequently, vector formats are useful for mapping and may be suitable for spatial distribution studies. Kvamme suggests that vector GIS are “...ideal for applications where the goal primarily is the viewing of spatial data in map form," (Kvamme 1989: 155). The output images provide researchers with an excellent visual representation for analysis. Unfortunately, vector GIS can be relatively expensive, limiting its availability (Maschner 1996b: 4). Furthermore, archaeologists often require the use of continuous data surfaces such as digital elevation models or topographic coverages, such as vegetation, soil surface or hydrology. Within vector-based GIS, data values have discrete point, line or polygon 
boundaries that only represent a small portion of the study area (Kvamme and Kohler 1988: 503). Therefore, elevation will be represented by contour lines, rather than a continuous, varied surface.

Raster-based GIS are based upon a grid system of cells. The area or map is divided into a series of equal grid cells and each individual cell is encoded with a particular value that is representative of some category of information (Knoerl 1991: 99). Raster GIS are relatively easy to program and have the unique ability to display maps of continuous data, creating high quality images, defined by grid resolution (Savage 1990: 24). With raster models, researchers can more readily identify variations within surfaces based on colour contrasts. Continuous characteristics such as soil type, topography, or elevation are well suited to raster models. Within a raster GIS, each cell in the grid will contain a unique value $(1,2,3 \ldots)$ for each data layer; the value corresponds to a particular classification (Kvamme 1989: 151). Raster-based analyses have been used most frequently by archaeologists. Raster-based systems are suited to location modelling, creating surface images ideal for manipulation and classification (Kvamme and Kohler 1988: 505). This is a significant advantage when analyzing the distribution characteristics of archaeological sites in a particular region.

While raster programs are very flexible, they can generate very large datasets and electronic storage space can become costly. Computer storage needs increase significantly as cell size decreases and require greater processing or computation time for analysis (Kvamme 1989: 151). While this can be problematic as the size of individual cells can affect the quality of information. Larger cells invite data generalizations or averages, which can smooth features and render them less pronounced or even invisible 
(Kvamme 1989: 152). If two or more classes are found within the boundary of a cell, the dominant class will generally be coded. As a result the “...true shapes and sizes of classes can be misrepresented, causing inaccurate area estimates and portrayal of category boundaries" (Kvamme 1989: 151-2). Researchers need to reach a compromise between an appropriate cell size and the number of data layers incorporated into the project. Vector and raster GIS have significant advantages and limitations. Vector data structures are generally better suited to the analysis of relational information, while surfaces are best analyzed with raster data structures (Fisher 1999: 5). Current GIS software make available both vector and raster data structures, allowing the user to determine which program is the most appropriate.

Once a suitable program has been chosen and the data have been entered, it is organized into separate layers, or individual coverages. Regional layers such as elevation, hydrology, topography, soil type, vegetation type or archaeological site locations can be stored separately and used to perform a variety of data manipulations (Wansleeben 1988: 436; Maschner 1996b: 2). Alternatively, the spatial distribution of artefacts and features can also be stored as individual layers corresponding to the archaeological levels for intra-site spatial analysis. Each layer is viewed as a horizontal surface, which is spatially referenced using the same coordinate scale. Co-registering multiple data layers can be difficult considering “...the various scales, coordinate bases, and projections that might occur in the original maps prior to GIS capture," (Kvamme 1989: 150). While it may be a lengthy procedure, using the same coordinate system is necessary for accurate comparisons and manipulations between different data layers. Intra-site spatial analysis 
should not have this problem unless data is being supplied from different excavations or different datum points are being used.

\section{4 - Advantages for Archaeological Applications}

There are currently three main areas of archaeological research that employ GIS, including spatial analysis, landscape archaeology and site location modelling for Cultural Resource Management (Savage 1990; Harris and Lock 1995). Archaeologists can take advantage of this new technology to increase the speed and efficiency of their evaluations. In recent years, there has been increased interest and application of GIS technology within archaeology and Cultural Resource Management (Green 1990: 5; Limp 2000: 232). GIS has become particularly useful for predictive modelling, for example, the prediction of potential site locations based on the premise that similar types of archaeological sites should be located in similar types of environment. Using GIS, a series of environmental variable maps are overlaid, such as hydrology, elevation, slope and vegetation. From these maps, the combination of environmental variables is recorded for known sites; areas on the map where similar combinations occur are then targeted as potential locations for new sites.

GIS has also been used to digitize archaeological sites at both the regional and intra-site level. Through digitizing, archaeologists can create computer-based images for the visual and analytical display of data. Continued development of GIS in archaeology should greatly expand the types of analysis it can address.

There are two basic applications of GIS in archaeological research. The first provides archaeologists with a data management and presentation toolkit for the 
collection, combination and presentation of relevant data. One of the principle advantages of GIS is the ability to store, manage and catalogue significant amounts of archaeological data, which can be easily retrieved and analyzed through exploratory and statistical investigation (Maschner 1996b: 12; Wheatley and Gillings 2002: 18). Geographic entities are represented as individual, unique spatial data structures within a GIS database. Archaeological information is also spatially referenced and is equally suited to storage within a GIS database at both intra-site and regional levels of resolution. As a result, it is possible to query individual archaeological data structures (for example, artefact locations on a site map) to access the attribute data maintained within the database (Stine and Lanter 1990: 80). This process allows researchers to access stored information quickly and efficiently.

The recovery of archaeological materials through excavation and survey can result in vast amounts of spatial and attribute data that need to be housed in an appropriate and accessible database system. GIS have the ability to store and retrieve a variety of data forms within a single database, which can then be integrated and manipulated in a variety of contexts. Information such as artefact or site location, digital terrain models and regional information can be easily updated or corrected and new data fields can be added to an existing database when necessary. Without the use of computer databases, archaeologists are limited to conventional, manual data analysis, which is often slow and time consuming. As a result, the number of variables or types of analysis that can be considered in a study is limited (Kvamme and Kohler 1988: 497). The use of manual methods for measurement and recording may also lead to the inclusion of 
variation and errors within a sample. Therefore GIS databases offer an appealing and time-saving alternative.

In addition to data storage and management properties, archaeologists can also take advantage of the excellent graphical properties of GIS applications combined with systems of quantitative analysis. This provides an ideal environment for spatial studies, including intra-site and regional analyses (Kvamme 1993: 91). Archaeologists often take advantage of the graphical capabilities of GIS to create detailed maps and visual displays. The spatial properties of archaeological sites and artefact information provide the researcher with the ability to produce maps of the landscape and identify unique relationships in the data. Within such programs, maps can be produced rapidly, incorporating suitable colour and shading, as well as three-dimensional perspectives, which are unavailable in conventional paper maps (Kvamme and Kohler 1988: 519). Computer generated maps can enhance site reports or publications, providing an excellent educational medium for research. Depending on the capabilities of both the available software and the user, there is unlimited potential for the creation and inclusion of new types of information (Kvamme and Kohler 1988: 498). Despite the functional capabilities of GIS, applications are most often restricted to data storage and management or the visualization of information without the benefit of research. While this is sometimes criticized as an ineffective use of the program, Fisher argues that the facility of mapping within a GIS is comparatively difficult with traditional software (Fisher 1999: 8). High quality maps produced with a GIS can be used to improve and strengthen the results of visual interpretation and methodology. 
As an alternative to data storage and visual display, GIS technology can be used as an effective research tool "...for the development of new and better ways of mapping and evaluating the archaeological heritage" (van Leusen 1995: 27). While the initial use of GIS in archaeology has emphasized its function as a database management tool, researchers are beginning to take advantage of the potential research properties associated with GIS. The introduction of GIS technology in archaeology has provided new means for the study of research questions involving social organization, spatial clustering and territoriality, “... which promises to advance the study of past social systems in relation to their physical and cultural environments"' (Savage 1990: 29). Therefore, the continued growth and diversity of GIS applications will lead to the development of improved, more effective research methodology.

GIS are capable of performing an impressive number of accurate and replicable measurements within a very short period of time. This allows relatively large databases to be analyzed entirely and successfully, instead of requiring the user to select a sample for analysis (Kvamme and Kohler 1988: 498). One of the unique functions of a GIS that is not shared with other database programs is an ability to query and overlay various types of spatial data, permitting the manipulation and generation of new information from the existing data. This can be a major cost-saving advantage, reducing the amount of time required for digitizing or encoding of information (Kvamme 1989: 156). For example, elevation information can be analyzed in a GIS to generate new maps containing information about aspect, slope or viewscape. The process of data query is termed Map Algebra and involves the use of mathematical functions such as subtraction or addition to create new data layers (Wansleeben 1988: 438; Kvamme 1989: 157). As a further 
example, two or more data layers can be added together to produce a new layer that contains all the features and attributes of each layer. These operations are unique to GIS and allow the user to identify and isolate individual features for the creation of layers designed to answer specific research questions.

Despite early success, GIS applications have been limited in archaeology. The power and capability of GIS technology would suggest that archaeologists have barely begun to exploit its full potential. GIS technology should not be considered as simply a new tool that facilitates current problem solving, but rather as a "...vehicle to address entirely new concerns or concerns for which we have been at a loss for answers," (Limp 2000: 232). Archaeologists need to expand the applications of GIS beyond data management and display to take advantage of the analytical capabilities of GIS software.

\section{5 - Potential Limitations}

While GIS have become a useful tool for the management and conservation of cultural resources, it is important to identify current and future limitations. The sophisticated nature of the technology has the potential to dictate research and the questions asked of the data (Gilbert 1991: 112; Ruggles and Church 1996: 149). Therefore, researchers need to consider the costs of equipment and technical training, as well as the quality of data analysis and the suitability of GIS in other disciplines. Only when these issues are addressed, will it be possible to evaluate the utility of GIS to provide fast, accurate and replicable information.

Initial applications of GIS in archaeology were hampered by the ongoing debate between inductive and deductive research (van Leusen 1995: 35). The initial acceptance 
of GIS technology was limited by the perceived problems associated with inductive research. Traditional, empirical information gathering allows researchers to confirm hypotheses and expectations. The use of modelling requires the exact opposite, allowing the evidence to create new expectations and interpretations through analysis (Zubrow 1990: 307). This dramatic change in thinking has often called into question the utility and accuracy of inductively derived models. Within archaeology, there has been "... almost mind-boggling preoccupation with hypothesis-driven deductive research, which has prevailed over exploratory, inductive research, even though it is no less problem oriented," (Maschner 1996b: 5). The scientific focus of the "new archaeology" in the 1960s advocated the use of deductive models, which involve a priori assumptions about the data. The main disadvantage of deductive methods is the justification of a priori assumptions; inductive methods rely heavily upon statistical methods that can be difficult to employ, however (Kincaid 1988: 555; van Leusen 1995: 35). Deductive models have greater utility for site interpretation, while inductive models have statistical precision. Therefore both models may have utility in archaeological research.

\subsection{1 - Costs and User Knowledge}

While the utility of GIS technology in archaeology has been demonstrated, one of the most important drawbacks is the high cost of equipment. Many institutions are forced to compromise the quality of GIS in order to afford the hardware and software (Wansleeben 1988: 450). This limits the variety of analytical functions that can be performed and may restrict the adoption of GIS to wealthy institutions, resulting in token availability and application within archaeology. In some situations, the expense of 
database development and management has curtailed the use of GIS in favour of more affordable tools able to perform similar tasks (Marozas 1991: 90). The acquisition of digital data can also be costly; collection and refinement of data can be the most timeconsuming and expensive phase of research. Data and equipment sharing can be an effective means of minimizing costs, while ensuring that databases are continually updated with new information. Zulick (1986: 327) argues that "the more that data, equipment and trained personnel are used, the further the costs, which may initially be high, are spread." Cost reductions are extremely important, especially within archaeology, where funding and time are often limited.

In addition to considerable expense, understanding the concept of GIS and making full use of its analytical properties can require significant training and research. While the use of GIS appears simple, the system's potential “...rests to a great extent on the user's experience" (Allen et al. 1990: 384). Archaeologists need to be experts in applying GIS technology to fully grasp its potential and limitations. Many of the concepts within GIS are complex and require dedicated training, suggesting that the majority of individuals exposed to GIS will develop a very rudimentary understanding of the principles (Guillot and Leroy 1995: 18). Therefore, the ability for individuals to effectively apply GIS to their research questions will ultimately depend on expanded efforts to train and educate. Those individuals with a genuine interest in the use of GIS in archaeology need to maintain a detailed understanding of the evolving potential and functionality of computing technology. This should be facilitated greatly by learning how other investigators are using these programs to explore unique research objectives. 


\subsection{2 - Quality of Data and Analysis}

While graphical representations provide an excellent medium for display, the results can lead to the creation of impressive, detailed graphics without true analytical value. The quality of mapping the source data is dependent on the methods of data collection in the field as well as in database information manipulation (Getter 1986: 143, Kincaid 1988: 556). Archaeologists are only beginning to computerize site information and many have discovered that records are incomplete, or inaccurate, e.g. the physical location of sites. Improvements in the quality of data collection, storage and analysis have been greatly enhanced by the design and incorporation of computerized databases. One of the benefits of a GIS database is the ability to visually identify errors in a particular database when the information is graphically displayed (Wheatley and Gillings: 2002: 83). Traditional databases do not have a spatial component and therefore errors generated through the recording and manipulation of data may not be realized, leading to inaccurate results. Researchers need to first consider the quality of data prior to evaluating the accuracy of the resulting analysis.

Recent applications of GIS have also suggested that archaeologists have uncritically applied deterministic or functionalist methods for site prediction and modelling. Environmental determinism is defined as “... a theoretical approach to archaeology that regards past (and present) cultures as somehow functions of, or shaped by, environmental pressures," (Gaffney and van Leusen 1995: 367; Fisher 1999: 9). Researchers are forced to view the landscape in a manner that effectively removes human experience and interaction (Llobera 1996: 613). Since the information that is considered for analysis influences results, this limits the types of questions that can be asked and the 
way that problems are evaluated (Kincaid 1988: 564; Gilbert 1991: 112). Researchers must consider the potential influence of cultural variables on the location of sites. The restriction of physical, environmental variables for the interpretation of archaeological materials clearly ignores the anthropological and archaeological aspects of human behaviour.

Llobera (1996) argues that archaeological studies can include environmental data without being deterministic. Determinism is the “...product of our interpretation as reflected through the way we use our information," (1996: 613). The future success of GIS technology within archaeology will be determined by its ability to develop appropriate research strategies that consider "...the complex temporal and spatial relationships in a landscape, and do so in human terms," (Gilbert 1991: 112). The development of cognitive and behavioural models within archaeological research will allow users to include investigations of gender, spirituality, sacred landscapes and ethnohistoric information (Dalla Bona 1993: 135, Maschner 1996a: 305; Ebert 2002). These considerations will be beneficial for the interpretation of resource significance and the development of more suitable models of site location and prediction. Suitability

Archaeologists have been known to adopt methodology and theory from other disciplines without critical evaluation. As a result, archaeologists may force their research to conform to the strength of the borrowed method, allowing it to dictate the types of questions and analysis that are possible. The development of GIS was not originally intended for archaeological applications and this has resulted in reduced functionality. For example, the definition of individual units of analysis in GIS is quite different from 
archaeology. Within raster GIS, units of observation are identified as individual cells at various scales of resolution, while archaeologists will usually identify individual sites as analytical units (Wansleeben 1988: 440, Kvamme and Kohler 1988: 499). The problem arises when attempting to combine both sites and cells in the GIS. For example, an individual cell would be considered as one unit in a GIS. Each cell could contain any number of archaeological sites depending on the size of the units defined, which may affect the results of analysis. Thinking critically about the data and chosen method can help identify and account for potential problems.

The rapid integration of GIS technology into archaeology has often been criticized for resulting in an absence of critical evaluation (Allen et al. 1990: 386). Researchers need to acknowledge that their questions are based on the archaeological record and the data should not be compromised in favour of the method. Gaffney and van Leusen (1995) associate current research limitations with the archaeologist's use of GIS, rather than the technology itself. Archaeologists and cultural resource managers cannot "...blame the GIS for being unable to do things it was not designed for" (Gaffney and van Leusen 1995: 371). Instead, archaeologists need to adapt GIS technology through the development of software to accommodate research needs. For example, this research adapts GIS technology for use in intra-site spatial analysis.

\section{6 - Three Dimensional GIS Applications}

Current GIS technology is limited in the kinds of quantitative analysis that are available for three-dimensional spatial data. Recent work has suggested that there should be more emphasis on the inclusion of visualization and quantitative analysis in spatial 
studies, as they are complimentary and essential for archaeological investigations (Kvamme 1993: 91; Kvamme 1995: 7). As previously mentioned, the graphical capabilities of a GIS are well suited to the presentation of spatial information. Kvamme argues that an effective graph is the key to conveying and interpreting spatial patterning (1995: 7). A distribution map of artefact clusters across a site provides the researcher with a visual representation of the statistical results of cluster analysis, for example. Through graphical display, results can be further interpreted with respect to the characteristics of the site, e.g. site features or slope. Graphical display combined with appropriate statistical analyses provide the most complete and effective results for archaeological spatial studies.

Despite the importance of quantitative analysis, one of the most notable deficiencies in current GIS software is the ability to adequately analyze threedimensional data. Limitations in multi-dimensional GIS present significant problems for the intra-site analysis of archaeological sites, where excavations involve the recording of materials in three-dimensional space (Maschner 1996a: 304; Harris and Lock 1995: 356). As a result, archaeological applications of GIS have generally been restricted to twodimensional analysis (Harris and Lock: 1995: 355; Fisher 1999: 8; Wheatley and Gillings 2002: 241). While this is useful for horizontal intra-site analysis and inter-site analyses of settlement patterning on a regional scale (Warren 1990; Altshul 1990; Csaki et al. 1995; Lock and Harris 1996), it places severe limitations on three-dimensional interpretations.

Traditional attempts to use GIS for three-dimensional analysis have required the investigator to "...construct, integrate and analyse within a stacked vertical series of twodimensional geographies" (Harris and Lock 1995: 355). While these types of analyses 
produce adequate information, they do not present the same quality of results. A true, three-dimensional analysis would provide the means for developing a better understanding of the history of occupation at a site, the structure and the interrelationships between stratigraphic units (Kvamme 1995: 8). Ideally, then, it would provide a means of quantifying the relationship between cultural data on the vertical plane.

The future of GIS in archaeology requires an improvement in three-dimensional analytical capabilities, which this research addresses. While GIS currently cannot be used independently for three-dimensional analysis, it can be combined with other spatial analytical programs and used successfully within archaeology as a database management and visualization tool. The statistical results of a three-dimensional spatial analysis can be incorporated into a GIS database to visualize and query spatial relationships in three dimensions. The graphical display capabilities of GIS, “... allows ready visualization of pattern in data, an essential adjunct to quantitative analysis," (Kvamme 1993: 102). Therefore a combination of GIS with a statistical program designed to analyze threedimensional data such as K-means as developed here, provides the investigator with an excellent tool for detailed spatial analysis and graphical presentation of results in three dimensions.

\section{7 - Future Developments}

Archaeologists have developed a greater appreciation for the potential problems and limitations of GIS technology. While there are a number of important issues limiting future success, the outlook remains optimistic (Kvamme 1989; Allen et al. 1990; Harris 
and Lock 1995; Maschner 1996a; Limp 2000). The power and capability of GIS in archaeology has only begun to be exploited. Maschner (1996: 303) argues that limitations in the use and development of GIS are obvious, but "... when problems of data quality, accuracy, computation, and the learning curve are weighted against the possible results, the knowledge gained outweighs the inherent difficulties."

Many of the functional problems encountered by archaeologists are the result of early stages in software development that will ultimately be improved through continuous refinement. The potential of GIS is only now being realized and the future will see the development of widespread, imaginative research in archaeology, with critical evaluation of both the dataset and the suitability of the methodology (Ruggles and Church 1996: 169; Fisher 1999: 10). The continued use of GIS should encourage specialization in research and technical development, critical evaluation of data collection methods and a significant reduction in costs. At present, the major limiting factor is knowledge. With continued development and innovative application, GIS will become familiar tools for archaeological research, particularly in the areas of Cultural Resource Management, landscape and spatial analysis. 


\section{- Chapter Four - \\ Introduction to the Dataset}

\section{1 - Introduction}

The potential benefit of Geographical Information Systems combined with an effective method of spatial analysis has been established. Most archaeological applications have focused on a two-dimensional analysis of the distribution of artefacts and fauna for the interpretation of intra-site activities, despite the meticulous recording and documentation of archaeological materials in three dimensions. Therefore it is necessary to make use of this important information, through the development of an appropriate strategy, which incorporates a three-dimensional dataset into a detailed analysis of artefact distributions. This chapter will provide a description of the region and site that will be examined in this study, including an outline of the potential problems that will be addressed through the application of a three-dimensional intra-site spatial analysis. The site of Karabi Tamchin offers a unique opportunity to study Middle Palaeolithic occupations of Crimea, which may reveal important information regarding Neanderthal occupations and the use of space in a rock shelter context.

\section{2 - The Crimea}

Situated on the north shore of the Black Sea, the Crimean peninsula (Figure 4.1 below) has a very long and diverse history of occupation, which has been the focus of numerous archaeological excavations (Stepanchuck 1993; Marks and Chabai 1998; Chabai and Monigal 1999; Hoffecker 2002). Most Middle Palaeolithic occupations in the Crimea are correlated with the final period of Neanderthal settlement, identified as the 
Middle Pleniglacial (OIS 3), from 55,000 to 25,000 years BP. More specifically, numerous occupation levels have been dated from 35,000 to 30,000 years $\mathrm{BP}$, when conditions in this region were milder (Hoffecker 2002: 66). However, as yet, little is known about settlement systems in the Crimea (Chabai, Marks and Monigal 1999; Marks and Chabai 2001). Continued excavation in the region will provide an opportunity to develop a better understanding of Neanderthal populations during the transitional period between the Middle and Upper Palaeolithic.

The Crimean peninsula represents the greatest concentration of Neanderthal occupations in Eastern Europe, which is attributed to the mild climate of the region and the increased preservation of archaeological sites (Hoffecker 2002; 78). The availability of water, lithic and faunal resources within the Crimean Mountain Ranges as well as the presence of natural cave and rock shelters created attractive living conditions during the Middle Palaeolithic. There are three ridges within the Crimean Mountains, which offer unique climatic conditions based on their altitude. The Main range rises to between 500$1400 \mathrm{~m} / \mathrm{asl}$, the Internal range rises to $400-500$ metres above sea level (m/asl) and the External range is between $200-300 \mathrm{~m} / \mathrm{asl}$ (Yevtushenko and Burke, In Press; Yevtushenko et al. In Press a and b). Middle Palaeolithic sites have been identified within the Main and Internal mountain ranges at varying frequencies. The majority of known sites have been identified within the Internal range. Yevtushenko and Burke (In Press a) argue that this could result from the interaction of two factors: 1) survey efforts have largely focused on the Internal range; and 2) there is an absence of quality raw materials for the manufacture of stone tools, in addition to a harsher climate in the Main Range. Therefore, the observed distribution of Middle Palaeolithic sites may result from 


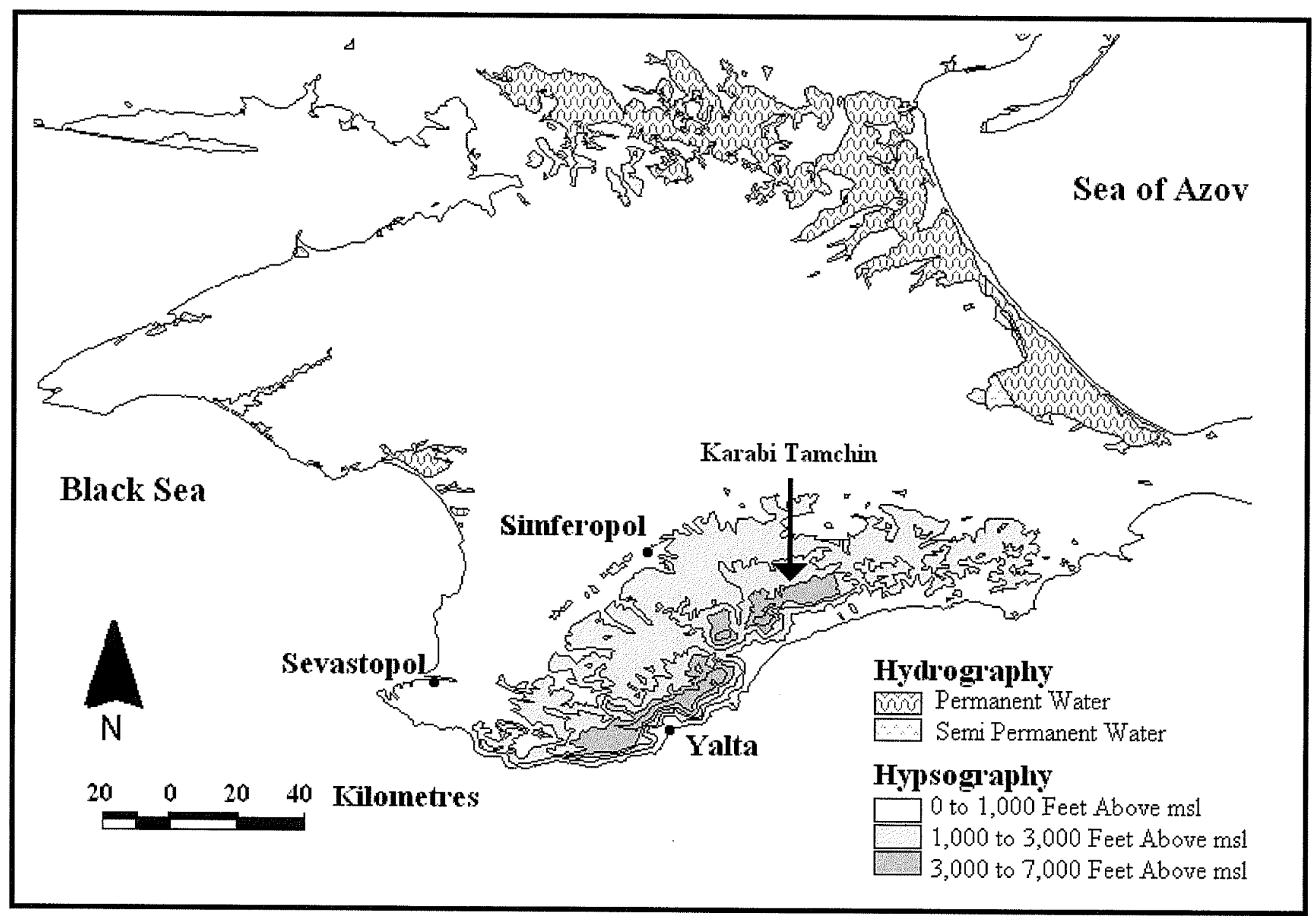

Figure 4.1: Map of Crimean Peninsula Including the Site of Karabi Tamchin (Identifed with Arrow) 
limited survey at higher altitudes or environmental conditions. This would further suggest that the discovery of archaeological sites in the Main Range would be extremely important to the development of a more complete understanding of the nature and distribution of Middle Palaeolithic occupations in the Crimea.

The location and interpretation of Palaeolithic occupations within the Crimea also has important implications for the development of the Palaeolithic record in Europe. While there are numerous Middle Palaeolithic cave and rocks shelter sites located throughout Western Europe, the majority of Eastern European sites are weakly stratified open-air deposits with limited preservation (Hoffecker 2002: 34). Therefore, the information obtained from cave and rock shelter sites in Eastern Europe has the potential to dramatically transform current perceptions of Middle Palaeolithic settlement within Eastern Europe.

One of the difficulties associated with the interpretation of spatial patterning in Middle Palaeolithic cave and rock shelter sites is the identification of distinct activity areas within a confined setting. Occupation floors within these types of sites generally contain a mixture of materials, which are difficult to interpret, whereas open-air sites tend to reveal clearer spatial information, as there are no spatial constraints (Straus 1977: 332). While it is difficult to interpret the spatial patterning of cave and rock shelter sites, these sites are critical to our understanding of Palaeolithic occupations. Furthermore, while open-air sites tend to be unstratified, caves and rock shelters often produce deeply stratified deposits ideal for vertical analysis. In addition, the conditions within cave and rock shelter sites are more amenable to the preservation of archaeological materials, as 
the "... exfoliation of the cave ceiling and walls creates a favourable geochemical medium for bones and teeth," (Hoffecker 2002: 119). Therefore, the potential information that can be recovered from these sites makes them an important resource for the development of prehistoric interpretations of the Palaeolithic in Eastern Europe.

\section{3 - Karabi Tamchin}

The site of Karabi Tamchin is one of only two known Middle Palaeolithic sites located within the Main Crimean Mountain Range and is the only stratified site excavated using modern techniques (Yevtushenko et al. In Press a). The site is a collapsed rock shelter at approximately $740 \mathrm{~m} / \mathrm{asl}$, which occupies the North Western edge of the Karabi plateau at the base of a vertical cliff, facing southwest. The site overlooks the Tamchin River, which is a tributary of the Suat River, located in the Audjikli ravine (Yevtushenko et al In Press b). Karabi Tamchin contains at least four distinct archaeological levels. Archaeological sites with well-stratified deposits provide the most suitable context for spatial analysis (Rick 1976: 133). Therefore, Karabi Tamchin contains valuable information for Middle Palaeolithic research in the Crimea regarding prehistoric behaviour, as well as expanding our knowledge of regional patterns of land-use.

A. Yevtushenko and V. Chabai originally discovered the site in 1996, with formal excavations occurring in six-week periods in 1999, 2000 and 2001. The site is a semicircular recess, which “... was covered by thick limestone slabs and blocks that appear to define the area covered by the former rock shelter," (Yevtushenko et al. In Press a). Two initial test units ( $1 \times 2$ metre) on either side of the recess identified Pleistocene deposits of approximately 1 metre deep containing Palaeolithic materials, 
which led to the excavation of a 27 square metre area in the centre of the site. Excavations were conducted using a $1 \mathrm{~m} \times 1 \mathrm{~m}$ alphanumeric grid system with elevation measurements recorded using a single datum. Artefacts and larger bone were recorded on hand-drawn maps at a scale of 1:10, which also included elevation points for those artefacts located three-dimensionally (Yevtushenko et al. In Press a and b). Within the lower levels of the site, the presence of carbonate cementation or brecciation required the use of an electric jackhammer for the recovery of archaeological materials. This also resulted in a decline in the number of artefacts and bone that could be recorded in three dimensions. In order to ensure maximum artefact recovery, all excavated sediments were sieved through $5 \mathrm{~mm}$ and $1.5 \mathrm{~mm}$ screens (Yevtushenko et al. In Press $\mathrm{a}$ and b).

The work at Karabi Tamchin has resulted in the recovery of a variety of lithic artefacts and faunal remains dating to the Middle Palaeolithic. Excavations and stratigraphic analysis have identified a sequence of cultural occupations separated by deposits of eboulis and loam materials (Yevtushenko et al., In Press a). A total of four in situ cultural levels have been recorded (see Figure 4.2) and form the basis of this research. Three radiocarbon dates have been obtained from faunal materials recovered in levels III, IV and V/2. Bone from level III produced a radiocarbon date of $>42,400$ uncal BP, while bone from level IV produced a date of $>41,200$ uncal BP and Level V/2 bone produced a date of $>29,800$ uncal BP (Yevtushenko et al. In Press a and b). These dates place Karabi Tamchin within the timeframe of the Middle Pleniglacial (OIS 3).

The uppermost archaeological levels contain a mixture of disturbed cultural materials that are not associated with Middle Palaeolithic occupations and will not be examined here. A variety of stone artefacts and fauna have been recovered from four in 
situ Middle Palaeolithic levels, a lithic analysis reveals that tool assemblages from levels II/2 and III can be associated with Western Crimean Mousterian (WCM) industries, while levels IV and V/2 have been identified as Crimean Micoquian (CMT) (Yevtushenko et al. In Press a). The Western Crimean Mousterian (WCM) typology includes Levallois and blade-core reduction, with an absence of bifacial tool technology. Typically, the WCM typology consists of side-scrapers, double-scrapers and various points. This typology has been identified elsewhere in sites mostly located in western Crimea (Yevtushenko et al. In Press a; Chabai 1998: 13).

The Crimean Micoquian (CMT) industry uses bifacial reduction, a predominance of unifacial scrapers and an abundance of tools with convergent edges (points, convergent scrapers) with moderate numbers of denticulate and notched tools (Yevtushenko et al. In Press a). This industry includes three different facies (Ak-Kayan, Staroselian and KiikKobaian) based on the presence and combination of certain tool types (Chabai and Marks 1999). The CMT has been located at several sites in the eastern part of the second range of the Crimean mountains (Yevtushenko et al. In Press a).

Faunal assemblages from each of the levels investigated here provide evidence for the exploitation of a variety of species, which would have inhabited the plateau, as well as the river valleys and cliffs south of the site. These include an extinct form of Asiatic ass, reindeer, saiga, antelope, red deer and chamois (Burke, In Press). Therefore, it seems that the inhabitants of Karabi Tamchin made use of a variety of local resources. This evidence provides important information regarding the nature of occupations at the shelter and will be considered in combination with the results of the k-means threedimensional spatial analysis (below). 


\section{4 - Site Stratigraphy}

Natural processes of soil formation, sediment deposition and erosion and weathering of the rock wall, in combination with cultural occupations at Karabi Tamchin have led to the accumulation of a complex stratigraphic profile studied by C. Reid Ferring (Yevtushenko et al. In Press a and b), which reveals important information about the nature of occupations at the site. Over time, the rock shelter would have been subjected to natural erosion, which is supported by the presence of irregular eboulis from the walls and the roof (Yevtushenko et al. In Press a). Excavation and subsequent examination of the geological profile at Karabi Tamchin reveals evidence for the chemical weathering of the limestone walls. Dissolution has led to the deep weathering of sediment and has resulted in carbonate cementation in the lower levels of the site. This process has occurred at varying degrees across the site and is a post-depositional process, which effectively crosscuts the original stratigraphic levels (Yevtushenko et al. In Press a). The process of cementation created significant difficulties for the excavation of the lower cultural levels and required modifications to the original excavation strategy. In turn, these modifications affected the collection and recording of archaeological materials in three-dimensions, which will be discussed in chapter 5 .

Cultural occupation levels were visually identified during excavation and can be associated with geological strata (Yevtushenko et al. In Press $a$ and b). Figure 4.2 displays the stratigraphic profile for the western wall of the site, along the square $8 / 9$ transect. Table 4.1 below displays the archaeological level and associated litholigical strata, which are identified in figure 4.2 . 


\section{Legend}

Archaeological Level $\mathrm{N} / 1$ - Lithological Strata $5 \mathrm{a}$ and $5 \mathrm{~b}$

II $/ 2-5 \mathrm{c}$

III - 6a and 7a

IV $-6 b$ and $7 b$

$V / 2-7 c$

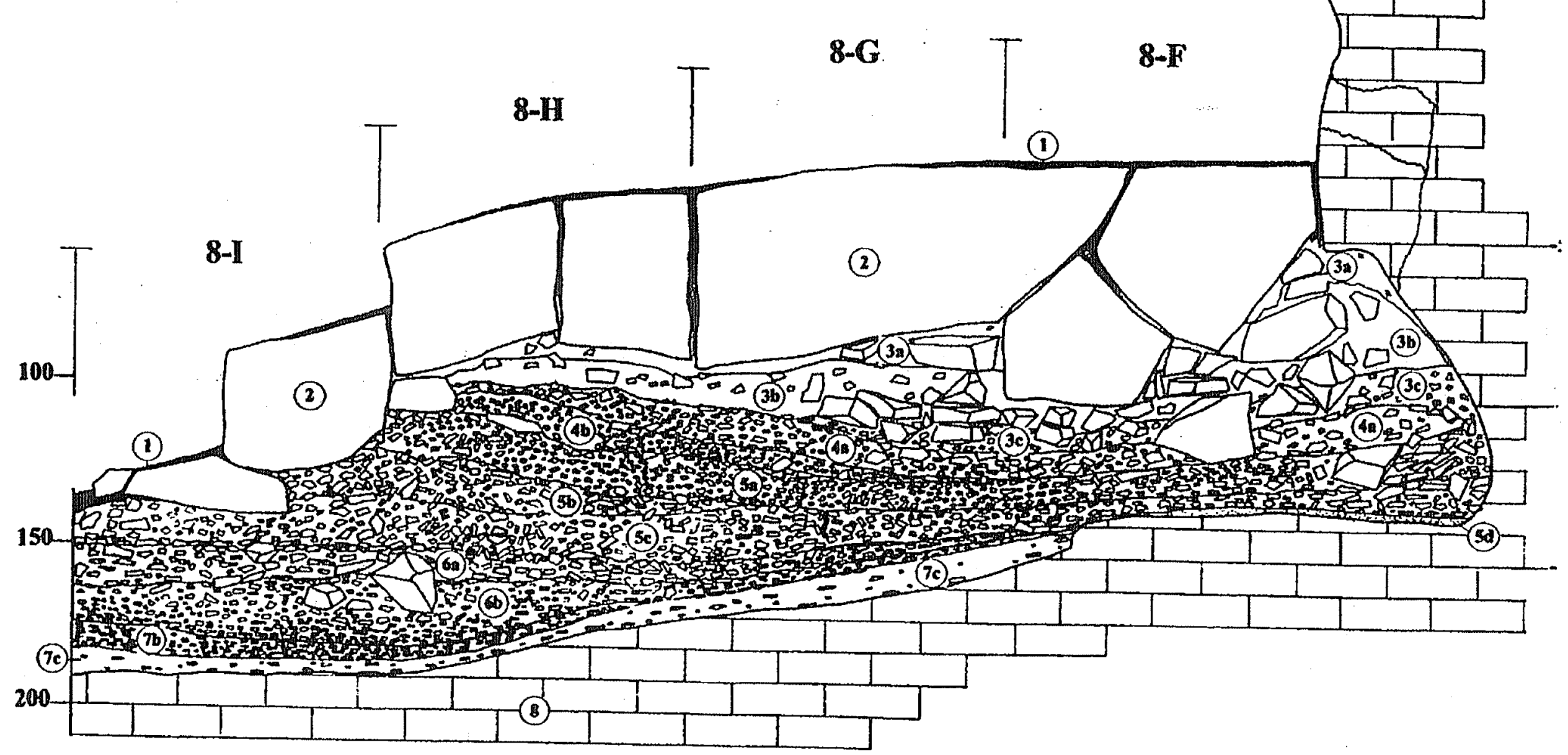

Figure 4.2: Stratigraphic Profile of Culturai/Natural Strata from Karabi Tamchin: West Wall Profile (Yevtushenko and Burke, In Press a) 


\begin{tabular}{|c|c|}
\hline Ar clineological & $\begin{array}{l}\text { Mithological } \\
\text { II Ievelts! }\end{array}$ \\
\hline $\mathrm{II} / 1$ & $5 a$ and $5 b$ \\
\hline $\mathrm{II} / 2$ & $5 c$ \\
\hline III & $6 a$ and $7 a$ \\
\hline IV & $6 b$ and $7 b$ \\
\hline$V / 2$ & $7 \mathrm{c}$ \\
\hline
\end{tabular}

Table 4.1: Corresponding Archaeological and Lithological Levels at Karabi Tamchin

The profile also identifies an interesting feature of the rock shelter, with respect to the position of the back wall. The back of the shelter was originally located in line with square $8-F$, as indicated in figure 4.2 by a small step in the bedrock. Geoarchaeological analysis has revealed that cultural levels IV and V/2 were deposited prior to the erosion of the back wall (Yevtushenko et al. In Press a). The shelter then proceeded to retreat into the wall of the cliff after the initial deposition of sediments. This resulted in a contraction of the shelter, but was also accompanied by an increase in the rate of erosion, leading to the deposition of greater concentrations of eboulis (Yevtushenko et al. In Press a and b). Levels II/2 and III were deposited at this time.

Differences in the depositional events of cultural layers at Karabi Tamchin are important for the interpretation of spatial patterning in three dimensions. There is a visible difference in the slope and soil characteristics of geological strata $6 \mathrm{~b}$ and $7 \mathrm{~b}$ and $\mathrm{c}$ (archaeological levels IV and V/2) compared to upper $6 \mathrm{a}$ and 5c (archaeological levels III and II/2). The lower strata are thinner deposits of finer sediments, which display a greater slope from north to south following the natural dip of the bedrock. Levels IV and V/2 were also subjected to varying degrees of carbonate brecciation across the site. Lithological strata associated with levels II/2 and III contain coarser sediments, with larger eboulis, but are relatively level. Topographic and sedimentological features of 
these levels will be considered when interpreting the results of the spatial analysis for each archaeological level (chapter 7).

\section{5 - The Dataset}

The three-dimensional information used for this study was recorded from handdrawn distribution maps of archaeologically defined levels that were drawn to a 1:10 scale, with $\mathrm{X}$ and $\mathrm{Y}$ coordinates recorded to the nearest centimetre. Artefacts (tools, cores and flakes $>2 \mathrm{~cm}$ in length) as well as large and identified bone fragments from each excavated 1 metre square unit were drawn onto plans during excavation. In addition, $Z$ values (depth below datum) were recorded for 1431 artefacts/bones ranging from -124 centimetres $(\mathrm{cm})$ to $-208 \mathrm{~cm}$ below datum, providing an adequate sample size for spatial analysis. This research uses only artefacts and faunal remains for which three-

dimensional coordinates were recorded. Artefacts for which only two-dimensional coordinates were recorded were not used, as they could not provide information on the vertical distribution of materials.

Table 4.2a and b, below, display the total number and proportional representation of three-dimensional archaeological materials. Each of the four archaeologically defined levels of interest contains a relatively high percentage of faunal materials, while level III has the highest percentage of stone tools.

\begin{tabular}{|c|c|c|c|c|}
\hline & \multicolumn{2}{|c|}{ 1.evelitive } & \multicolumn{2}{|c|}{ 1sevel 1111} \\
\hline Iritefact TYpes & Mrequency. & Percent & Mrequency & Perenent \\
\hline Bone & 237 & 58.7 & 125 & 46.6 \\
\hline Stone Flakes and Chips & 116 & 28.7 & 96 & 35.9 \\
\hline Stone Tools & 51 & 12.6 & 47 & 17.5 \\
\hline Total & 404 & 100.0 & 268 & 100.0 \\
\hline
\end{tabular}

Table 4.2a - Total Artefact Frequency and Percentage Distribution for Level II/2 and III 


\begin{tabular}{|c|c|c|c|c|}
\hline & \multicolumn{2}{|c|}{ 1.0\%ery } & \multicolumn{2}{|c|}{ 16ever. } \\
\hline Arrefret TYus & Mrequerro & 1\%oral & Wregneney & Poron \\
\hline Bone & 239 & 54.2 & 140 & 46.3 \\
\hline Stone Flakes and Chips & 163 & 37.0 & 118 & 39.1 \\
\hline Stone Tools & 39 & 8.8 & 44 & 14.6 \\
\hline Total & 441 & 100.0 & 302 & 100.0 \\
\hline
\end{tabular}

Table 4.2b - Total Artefact Frequency and Percentage Distribution for Level IV and V/2

The three-dimensional information for each level was entered into a computer database containing the level and square designation, the type of material (bone, stone flake, chip or tool) and the corresponding $\mathrm{X}, \mathrm{Y}$ and $\mathrm{Z}$ coordinates for each artefact or bone. The completed database is used to perform the K-means cluster analysis and will provide the necessary attribute information for the horizontal and vertical analysis of artefact distributions.

\section{6 - Potential Problems to be Addressed}

Preliminary research at Karabi Tamchin has revealed low artefact densities within each cultural level, which is likely a reflection of short occupation periods at Karabi Tamchin (Yevtushenko et al. In Press a). This information may indicate the potential presence of palimpsests within the shelter (as discussed in Chapter 2). Spatial analysis will be used to demonstrate that the shelter was indeed used for four successive short term occupation by small groups, rather than one, continuous long-term occupation. In order to do this it is necessary to develop an appropriate methodology designed specifically for the identification of these types of deposits. Spatial analysis in archaeology has traditionally focused on either the horizontal plane, or the vertical dimension. As the horizontal distribution of artefacts provides useful information regarding the distribution of activities within individual cultural levels, one must be 
certain that the artefacts are associated with a single occupational phase contemporary with the level being analysed. Therefore, any analysis of spatial patterning should consider first how the vertical dimension has influenced site formation processes and the possible post-depositional movement of materials.

This thesis establishes a methodology for the objective identification and analysis of the spatial distribution of: 1) cultural levels and 2) archaeological finds within levels. This investigation focuses on the identification and interpretation of stratified cultural layers from the collapsed rock shelter of Karabi Tamchin, where the stratigraphy is complex and the existence of at least some palimpsest deposits is suspected.

\section{7 - Conclusions}

The site of Karabi Tamchin potentially contains important information about Neanderthal behaviour in general and more specifically, in the Crimea. The characteristics and problems associated with the analysis of Middle Palaeolithic rock shelter sites have been identified (above), including the potential presence of palimpsests. The importance of three-dimensional analysis within archaeology has also been indicated. It is now necessary to account for these potential limitations with the application of a three-dimensional spatial analysis that combines the quantitative results of K-means cluster analysis with the graphical and analytical capabilities of GIS. Chapter 5 will provide a detailed description of the methodology developed here in order to investigate and interpret artefact distributions in several archaeological levels from Karabi Tamchin. Once the vertical distribution of levels has been established, intra-level spatial patterning (of the distribution of individual clusters) will be examined. This will 
provide an opportunity to interpret the behavioural patterning of Neanderthal populations, as seen through the distribution of archaeological materials at Karabi Tamchin. 


\section{- Chapter 5 - \\ Methods}

\section{1 - Introduction}

Within archaeology, the use of spatial analysis can reveal important information regarding the types of activities that occurred within a particular site as well as how those activities were organized in terms of space. The preceding chapters have demonstrated the significance of spatial analysis in archaeology as well as the potential benefits of Geographic Information Systems as a visualization and database management tool. Kmeans cluster analysis has been successfully applied in archaeology to identify spatial patterning in three-dimensions. This chapter describes the methodology developed for the three dimensional spatial analysis of artefact and faunal distributions at Karabi Tamchin. After the dataset has been transformed for use in the GIS programs, the entire database is examined initially as a test of the integrity of cultural levels identified during excavation. In addition, individual archaeologically defined levels are analyzed in order to identify clusters of finds that may signify possible activity areas within the site. This is accomplished by using ArcView 3.2 GIS software to visualize horizontal spatial patterning and ArcGIS to visualize three-dimensional distributions, followed by cluster analysis to identify discrete levels and intra-level spatial organization; and finally, statistical analyses of cluster contents to identify potential activity areas.

\section{2 - Test of Randomness}

As an independent check that the three-dimensional dataset is an unbiased sample of the total dataset distribution, the distribution of all faunal remains for each archaeologically defined level was plotted on a grid, based on frequency counts for each 
excavated 1-metre square. Separate grids were created for total bone concentrations (Appendix A - Figures 1-4), total identified bone (Appendix A - Figures 5-8) and the total burnt bone concentrations (Appendix A - Figures 9-11). A grid for unidentified bone was also produced, which included unidentified bones, bone flakes and tooth fragments less than $2 \mathrm{~cm}$ in length and those between 2-5 cm and 5-10 $\mathrm{cm}$ (Appendix A-

Figures 12-15). Next, plots of all three-dimensionally referenced bone were produced for each level and compared with the distributions (Appendix B - Figures 4, 8 and 12). If the three-dimensional dataset displayed similar distribution characteristics, it would suggest that the distribution of finds in the entire database has been randomly sampled and the three-dimensional data could be used for the interpretation of intra-level activities.

Comparisons between the total faunal distributions (identified and unidentified materials) and the three-dimensionally recorded distribution demonstrated similar patterning for each level, indicating that the dataset is an unbiased representation of the total faunal distribution and the research can proceed. Burned faunal distributions for levels III and IV will be discussed in chapter 7 and used to interpret the possible location of hearths in relation to the distribution of artefact clusters.

\section{3 - Re-Sampling Strategy}

The excavation of Karabi Tamchin occurred over a period of 3 consecutive field seasons $(1999,2000$ and 2001). As discussed in chapter 4, excavations in the 1999 season encountered several difficulties including the presence of breccia, or varying degrees of carbonate cementation within the lower geological strata including part of archaeological level III, IV and all of level V/2. As a result, excavation strategies were modified in 
subsequent years including the use of an air-hammer to loosen sediments before removal and sieving. Due to this change in excavation strategies, a significantly higher percentage of archaeological materials were recorded in three dimensions in 2000 and 2001. Figure 5.1 (below) displays a plot of the total artefacts recorded in three dimensions throughout the entire excavation; transects 9,10 and 11 excluding row $\mathrm{J}$ were excavated in 1999. An interpretation of spatial patterning using k-means cluster analysis would create clusters that are biased by the density of artefacts towards the outer squares of the site. As a result, clusters would be representative of different excavation strategies, rather than the natural spatial organization of archaeological materials within the site. Therefore, a resampling strategy is devised to minimize the problems associated with excavation differences.

A visual examination of the dataset indicates that there is an obvious difference in artefact densities along the 8/9 transects and the 11/12 transects in each archaeologically defined level. Therefore, the number of artefacts was counted along either side of lines $8 / 9$ and $11 / 12$ lines (excluding row J) and counts were used to produce a re-sampling ratio to use when re-sampling the units excavated in 2000 and 2001. Artefacts within $20 \mathrm{~cm}$ on either side of the transects were counted to produce the ratio. Unfortunately, this revealed that the presence of carbonate cementation in level $V / 2$ has greatly affected the recovery of three-dimensional information and the possibility of interpreting the natural spatial patterning of artefact distributions is limited. Therefore, the level V/2 dataset is excluded from the cluster analyses. In addition, the number of three-dimensional artefacts recovered for archaeological sub-level 2-1 was limited in the original distribution and further reduced by the re-sampling procedure, thereby eliminating it from further 
analysis. Table 5.1 (below), displays the ratio calculations for levels II/2, III and IV and the final percentage of materials to be re-sampled from the 2000/2001 excavations.

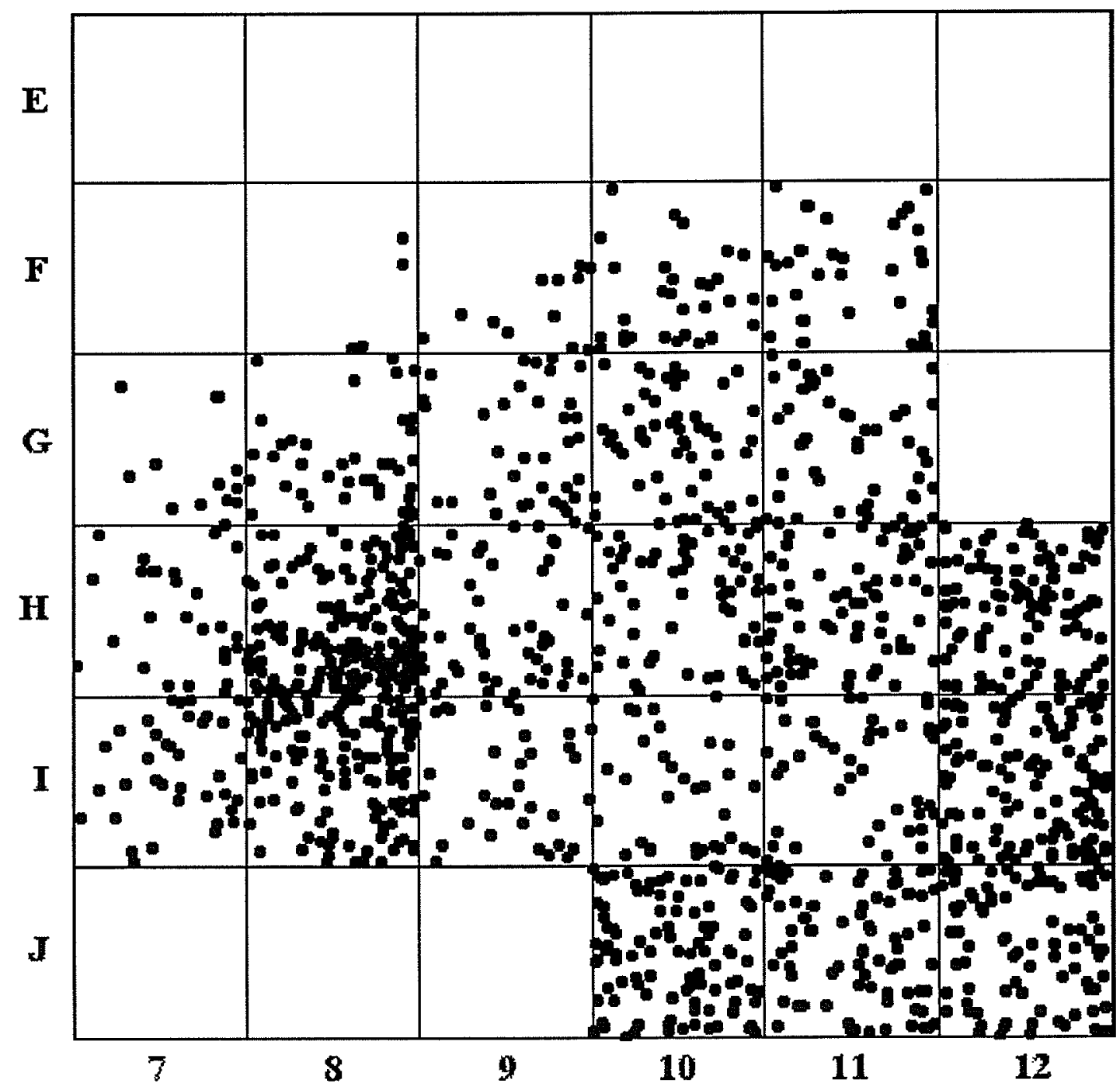

Figure 5.1: Total Three-Dimensional Artefact Distribution for Karabi Tamchin (transects 9, 10 and 11 excluding row J, were excavated in 1999). 


\begin{tabular}{|c|c|c|}
\hline Archaeological & Totoln Ratio & 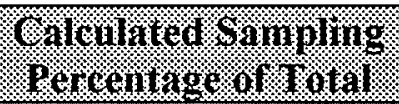 \\
\hline $\mathrm{II} / 2$ & $61: 14$ & $23 \%$ \\
\hline III & $33: 26$ & $85 \%$ \\
\hline IV & $58: 21$ & $36 \%$ \\
\hline
\end{tabular}

Table 5.1: Calculated Artefact Frequency Ratios and Sampling Percentages for Levels II $/ 2$, III and IV

The resulting ratios were used to re-sample the $2000 / 2001$ excavated materials; $100 \%$ of the materials from the 1999 excavations were combined with the re-sampled data to create a sampled dataset for each level. Re-sampling was performed three times for each level and the resulting datasets were analyzed using k-means cluster analysis. The horizontal distribution of each trial is compared to ensure that re-sampling is unbiased.

A series of chi-square significance tests were performed for each level to determine if the frequency and percentage of artefacts in each trial were significantly different. This was used to determine whether the sampling technique could produce three sets of random artefact distributions to be used for the k-means cluster analysis. The results would also make it possible to identify whether the results were similar enough to warrant selecting one solution for analysis and interpretation. The chi-square significance tests determined that none of the three trial comparisons were able to reject the null hypothesis at the required .05 level of significance (Appendix D - Tables 1-9). It is also important to note that the resulting chi-square values were very low in each comparison, which indicates that there is almost no difference between trials. Therefore, the chisquare statistical results indicate that each trial is comparable for each level, supporting the selection of one trial for interpretation. 


\section{4 - GIS and Three-Dimensional Visualization}

One of the drawbacks of ArcView 3.2 is an inability to display information in three dimensions in an adequate fashion. However, it is still useful for two-dimensional visualization and provides powerful analytical capability for database query. To view data in three dimensions in ArcView 3.2, the 3D Analyst extension can be used. The 3D Analyst extension converts two-dimensional data into a three-dimensional visual display; however it does not provide a good display when the range in vertical distribution is relatively small (Booth 2000: 177). The vertical extent of the dataset for this study is 84 $\mathrm{cm}$. When the data is displayed in three dimensions using the 3D Analyst, the vertical range is too small to distinguish clusters visually with any accuracy. Another limitation of the $3 \mathrm{D}$ analyst is the absence of a reference grid, rendering it impossible to determine the angle at which the data are being viewed.

ArcGIS, a more recent GIS program than ArcView 3.2, offers two extensions for the analysis of 3 dimensional data and is therefore used in this research for the presentation and visualization of three-dimensional data, which is used when interpreting the results of the cluster analysis. The Geostatistical extension provides an excellent way to visualize and analyze three-dimensional data. The trend analysis function is also an extremely useful tool for visualizing data in three dimensions. A dataset is displayed with the $\mathrm{X}, \mathrm{Y}$ and $\mathrm{Z}$ axes visible, making it easy to view data on three different planes. It also identifies global trends, which can provide information concerning the slope. The main advantage of the trend analysis is its ability to display 3 dimensional data on a spinning 3D plot. The dataset can be rotated horizontally in 360 degrees, and can be rotated vertically in 180 degrees. With such a wide range of angles, it is possible to locate trends 
in the data from any viewpoint. For this research, the entire dataset distribution (all levels) is examined using the geostatistical trend analysis to examine both vertical clustering in the dataset as well as global trends.

\section{5 - K-Means Cluster Analysis}

A statistical method of identifying three-dimensional clusters is required to provide an objective test of subjective visual analysis. The statistical method chosen for this project is the k-means cluster analysis, available in both SPSS version 10.0 and Kintigh's (1994) Tools for Quantitative Archaeology (TQA). The procedure is relatively well understood and accessible, offering a simple, yet effective method for the identification and interpretation of spatial data (see chapter 2). One of the main advantages to this approach is its ability to handle large data sets, which are quite common within archaeology (Ammerman et al 1983: 212).

$\mathrm{K}$-means cluster analysis is defined as an equal variance method, i.e. it searches for clusters with equal variance in all dimensions. The resulting analysis attempts to produce ideal spherical clusters; although this is not always appropriate when considering archaeological levels (Koetje 1992: 178). For example, normal, spherical clusters are unlikely to provide adequate representation of the vertical distributions of archaeological levels. The analysis of three-dimensional distributions presents a unique problem for equal variance procedures. Within archaeology, distributions along the vertical dimension are generally much more restricted spatially than horizontal ( $\mathrm{X}$ and $\mathrm{Y})$ distributions. Without a consideration of distribution differences between the three axes of variation, greater weight will be assigned to the spatial axis with the greatest variation (Koetje 
1991: 189). Despite these difficulties, a series of $Z$ coordinate transformations (discussed below) can be applied to the dataset, allowing the k-means clustering analysis to produce comprehensive and meaningful results in three dimensions. The program can also be used in combination with GIS software, to provide a quantitative analysis, which is easily visualized and interpreted. Two packages are available to test the successful application of k-means for three-dimensional analysis. A series of experimental trials were run using both SPSS v.10 and Kintigh's (1994) program. Results of the k-means cluster analysis in SPSS v.10 and the TQA program were compared (see below) to determine which method is the most appropriate for the Karabi Tamchin dataset.

\subsection{1 - SPSS v.10}

The SPSS v.10 k-means program was used as an initial test of the integrity of archaeologically defined levels. One of the benefits of the SPSS v.10 k-means cluster analysis package is the option it provides to save the cluster membership of each point as a column in the original database. By saving the cluster membership, the database can be queried by individual cluster, making it possible to examine the attributes of points within each cluster. Once the cluster analysis is performed and the cluster membership of each point is saved, it is possible to query the database in ArcView and ArcGIS to determine the vertical distribution for each cluster to compare with the $\mathrm{Z}$ value distribution of the archaeologically defined levels.

A drawback of SPSS v. 10 is that in order to run a k-means cluster analysis in SPSS v. 10, it is necessary to specify the number of clusters in the dataset. This is done using the point distribution and the trend analysis function in ArcGIS. This requirement 
was deemed too subjective and it was decided that Kintigh's Program would be used for the remainder of this research.

\subsection{2 - Kintigh's Tools for Quantitative Archaeology}

As previously stated, an important limitation of the k-means program in SPSS v. 10 is the requirement for a user-defined number of clusters. This results in subjective interpretation of the data and does not allow the program to determine a statistically appropriate number of clusters based on the actual dataset. This is especially problematic when it is necessary to identify natural clusters in the dataset, based on the points themselves. Kintigh's Tools for Quantitative Archaeology (Kintigh, 1994) offers an appropriate alternative, as the computer program statistically determines the most appropriate number of cluster solutions based on the original dataset. Trial runs of Kintigh's program were performed using the entire dataset for Karabi Tamchin. In addition, Kintigh's program (1994) is used to perform a three-dimensional cluster analysis of each of the archaeologically defined levels II/2, III, IV and V/2 in order to identify possible palimpsests and interpret spatial organization at Karabi Tamchin (the results are in chapter 6).

The k-means module of Kintigh's program requires the user to create simple text files, which contain the $\mathrm{X}$ and $\mathrm{Y}$ coordinates for each point in the analysis. The program also requires the user to determine the clustering range for a particular dataset. For each trial run, including the entire dataset and each of the 4 archaeologically defined levels, a clustering range of 1-20 clusters was chosen. This provides the program with a range that is diverse, yet reasonable, given the size of the dataset, the excavation area and the type 
of site (rock shelter) being investigated. The program requires the user to identify the number of random runs required for the dataset. A total of 10 random runs were selected for each trial to provide useful comparisons with the original dataset results.

\section{6 - Data Preparation}

\subsection{1 - Mapmaker Pro and ArcView 3.2}

The final database of three-dimensionally recorded objects for levels II/2, III, IV and 5 contains information including the $\mathrm{X}, \mathrm{Y}$ and $\mathrm{Z}$ coordinate, the type of artefact represented by that point and both the archaeological level and unit number from which the artefact was recovered. It is also necessary to transform the vertical distribution of finds into a scale that is easy to visualize and interpret in three-dimensional space. This becomes important when interpreting the results of the cluster analysis. The original vertical scale of $-124 \mathrm{~cm}$ to $-208 \mathrm{~cm}$ below the surface was converted to a scale of $-1 \mathrm{~cm}$ to $-84 \mathrm{~cm}$ and recorded in the database. A separate database was created for each of the 4 defined levels and merged into a single relational database.

It is necessary to convert the spatial dataset for use in GIS through the creation of a series of shapefiles. A number of data transformations are required to plot the points accurately so they correspond to the original archaeological grid. When the original points are recorded, each excavated square is assumed to range from zero to $100 \mathrm{~cm}$ on the $\mathrm{X}$ and $\mathrm{Y}$ axes. If the $\mathrm{X}$ and $\mathrm{Y}$ coordinates were entered into the database unchanged, the computer would read all of the points as belonging to the same $1 \mathrm{~m} \times 1 \mathrm{~m}$ square and points would not be separated into the appropriate squares. As seen in Figure 5.2 below, the site grid consists of 6 units across ( $\mathrm{X}$ axis) and 5 units down ( $\mathrm{Y}$ axis). 


\begin{tabular}{|c|c|c|c|c|c|}
\hline $7 F$ & $\mathbf{8 F}$ & $\mathbf{P F}$ & $10 \mathrm{~F}$ & $11 F$ & Not Excavated \\
\hline $7 \mathrm{G}$ & $8 G$ & $9 G$ & $10 \mathrm{G}$ & $11 \mathrm{G}$ & Not Excavated \\
\hline $7 \mathrm{YH}$ & $8 \mathrm{PH}$ & $9 \mathbf{H}$ & $10 H$ & 11H & $12 \mathrm{H}$ \\
\hline $7 I$ & 81 & 91 & $10 I$ & 11 I & 121 \\
\hline Hot Excavaled & Not Excavatod & Not Excerated & $10 \mathrm{~J}$ & 11J & $12 \mathrm{~J}$ \\
\hline
\end{tabular}

1 Metra

Figure 5.2: Excavated Grid of $1 \mathrm{~m} \times 1 \mathrm{~m}$ Square Units at Karabi Tamchin $25 \mathrm{~m}^{2}$

Each square unit represents $1 \mathrm{~m}^{2}$, resulting in an $\mathrm{X}$ scale of 0 to $6 \mathrm{~m}$ and a $\mathrm{Y}$ scale of 0 to $5 \mathrm{~m}$. As the squares increased from left to right, $1 \mathrm{~m}(100 \mathrm{~cm})$ was added to the original $\mathrm{X}$ coordinates and as squares increased from the front to the back of the cave, $1 \mathrm{~m}$ $(100 \mathrm{~cm})$ was added to the $\mathrm{Y}$ coordinates. The resulting transformations are displayed in Figure 5.3. For example, points within square $10 \mathrm{~J}$ would have $400 \mathrm{~cm}$ added to the original $\mathrm{X}$ coordinates and $0 \mathrm{~cm}$ added to the $\mathrm{Y}$ coordinates. These changes are recorded in the database as a separate column so that each point entry contains both the old and the modified $\mathrm{X}$ and $\mathrm{Y}$ coordinates. Changes were checked against the grid and the original coordinates to ensure accuracy. 


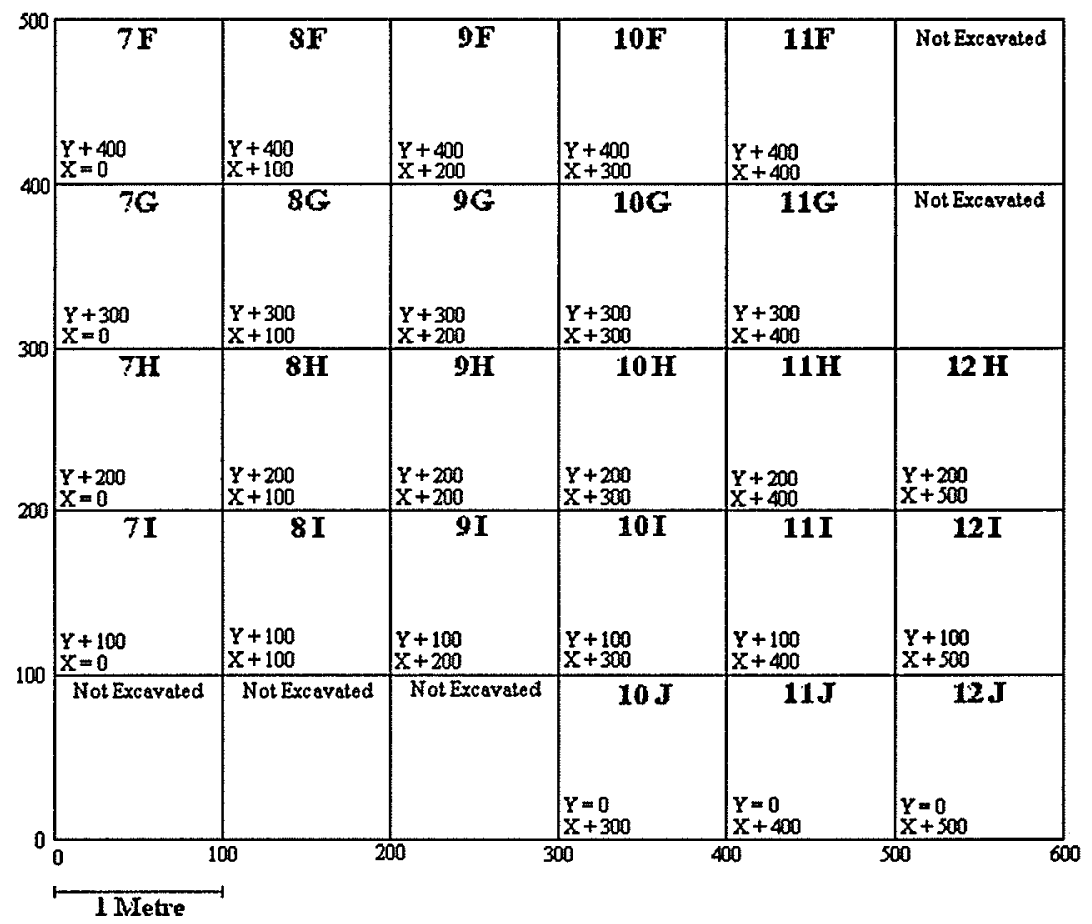

Figure 5.3: $X$ and $Y$ Coordinate Transformations for Archaeological Grid $25 \mathrm{~m}^{2}$

As a visual check of the database to determine that all of the points were accurately mapped according to the archaeological grid, the database for each level was viewed in Mapmaker Pro. Mapmaker Pro is a relatively simple mapping program that can create files to be exported in a number of different formats for use within more complex programs. Using Mapmaker, a point file is created for each of the 4 defined levels as well as one point file that combines all levels. In addition, a reference grid of 6 columns and 5 rows, each with a horizontal and vertical extent of $1 \mathrm{~m}(100 \mathrm{~cm})$ is used to test the point files. By overlaying each of the level point files onto the reference grid, it is possible determine if the points are accurately mapped. Once each point file is evaluated, the files are saved and exported as shapefiles to be used within ArcView GIS 3.2 for the twodimensional (horizontal) analysis. Files are exported together with the point distribution image and its corresponding database. The grid file is also exported with a database 
containing the unit identification and the $\mathrm{X}$ and $\mathrm{Y}$ scale ranges for each square. Once the files are exported as shapefiles, they can be opened using ArcView 3.2, or ArcGIS for both two-dimensional and three-dimensional analysis. When the Grid image is created in Mapmaker, arbitrary identifications are assigned for each grid cell. Using the edit table function in ArcView, each of the grid cells is altered to correspond with the appropriate archaeological square designations and a table join is performed between the grid and each corresponding level to allow each of the points to be referenced with the appropriate grid square.

Figure 5.4a, b, c, and d (below) illustrate the archaeological shapefiles for levels II, III, IV and V/2, including the individual point file and the reference grid. Once the shapefiles are evaluated against the original dataset to ensure accuracy, individual artefacts are colour-coded to provide an initial visual interpretation of the general spatial trends for each level (Appendix B).

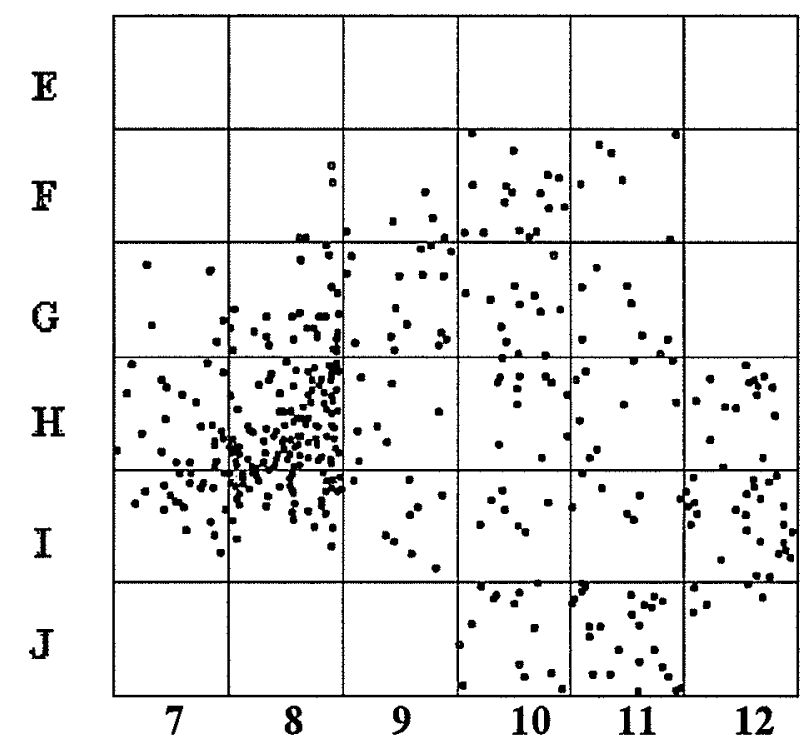

Figure 5.4a: Level II Shapefile

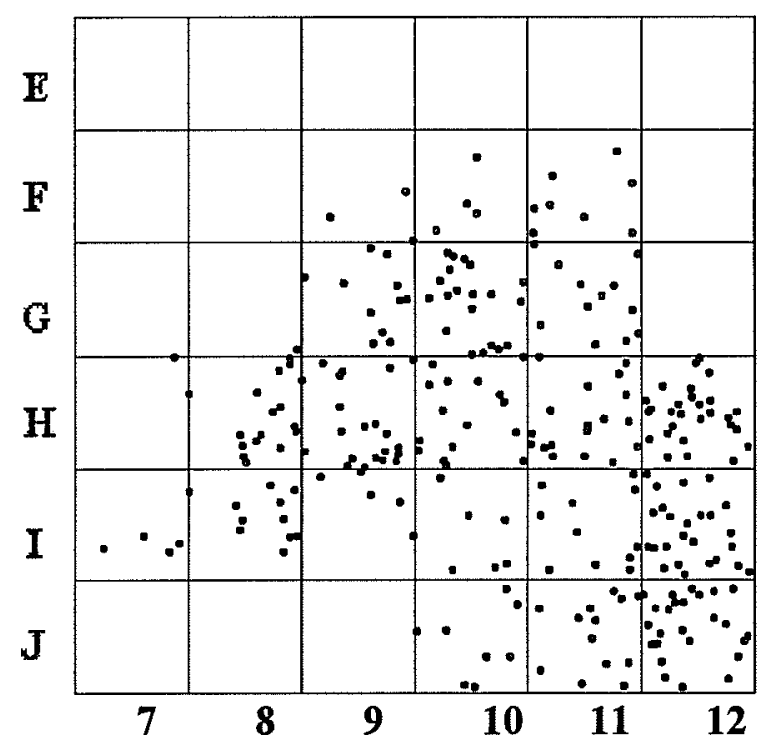

Figure 5.4b: Level III Shapefile 


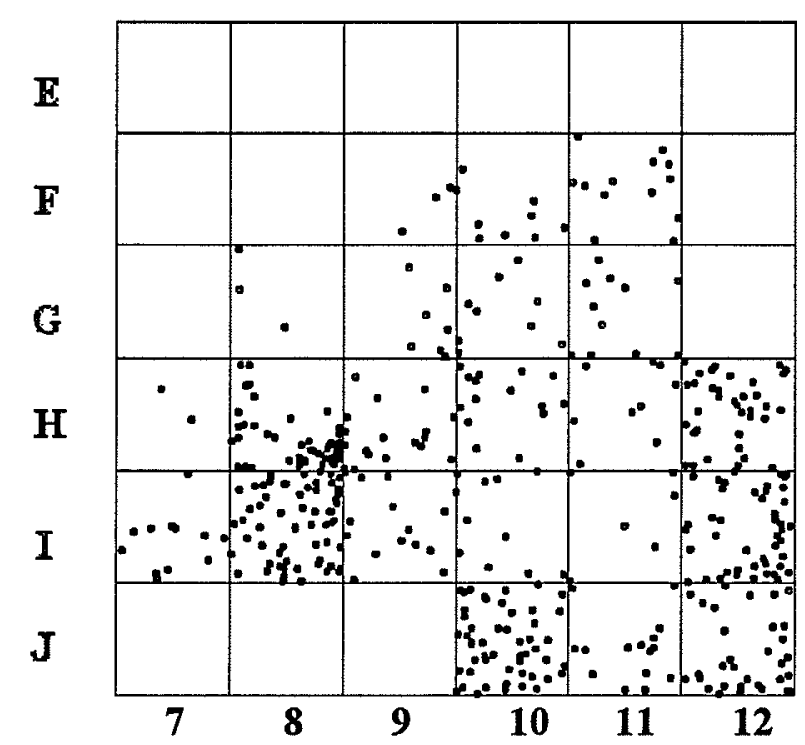

Figure 5.4c: Level IV Shapefile

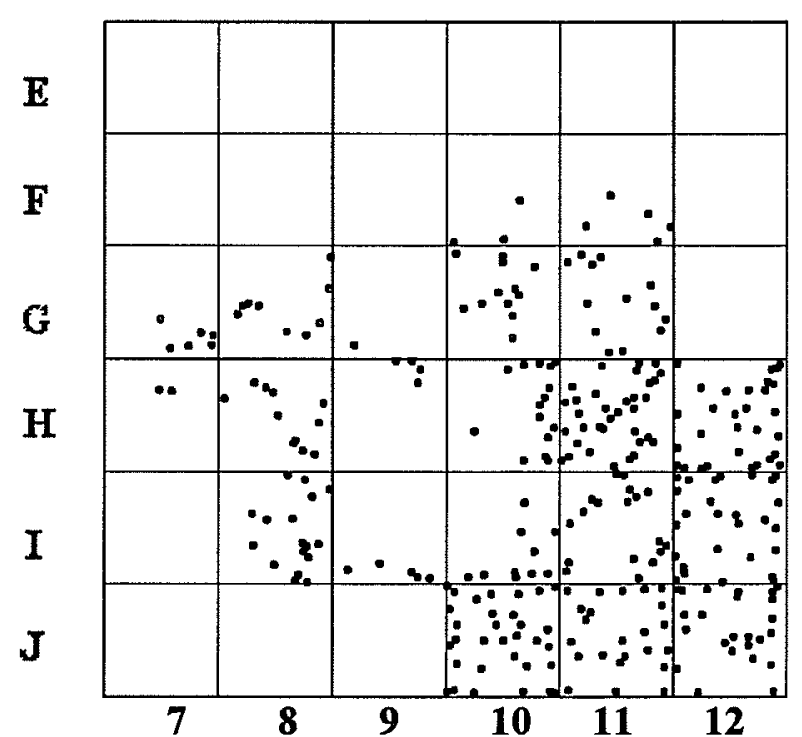

Figure 5.4d: Level V/2 Shapefile

\subsection{2 - K-means Analysis Using Kintigh's Tools for Quantitative Archaeology}

In order to create an appropriate dataset for three-dimensional clustering (one that creates equal variation in three dimensions), a series of transformations are applied to the original dataset (see below) and the results are compared to determine the relative effectiveness of each procedure. An initial two-dimensional cluster solution $(\mathrm{X}, \mathrm{Y})$ is performed and the results are compared with the three-dimensional clustering tests. Given the concerns expressed by Koetje (above) regarding the effects of the k-means equal variance assumptions, which create spherical clusters and lead to compression on the vertical scale, a $Z$ scale transformation must be chosen. Three data transformations were applied to the $\mathrm{Z}$ coordinates of the dataset for each level to evaluate their effectiveness for the analysis of clusters in three dimensions. The transformations applied to the Karabi Tamchin dataset include simple $\mathrm{Z}$ expansion factors of 5, 10,15 and 20, $\mathrm{Z}$ score or data standardization and a combined trial of the $Z$ expansion factors and data standardization. 


\subsubsection{1 - Z Expansion Factors}

A $Z$ axis expansion factor is used to improve the discrimination between the horizontal and vertical dimensions in the dataset. The application of an expansion factor places additional emphasis on the $Z$ axis and can actually improve the ability to discriminate between various layers when the vertical distribution is small (Koetje 1991: 189, Koetje 1990: 267). A series of expansion factors $(5,10,15$, and 20) were applied to the $\mathrm{Z}$ coordinates for each level to determine which factor was most suitable. A separate column was created in the database for each expansion factor and the original $\mathrm{Z}$ coordinates were multiplied by the various factors and saved. An appropriate $Z$ expansion factor improves the range of $Z$ coordinate values so that they are comparable to the range in the $\mathrm{X}$ and $\mathrm{Y}$ coordinates. This places equal importance on all three dimensions, and creates k-means clusters, which are representative of the data in three dimensions.

\subsubsection{2 - Data Standardization Trial}

An object's original spatial coordinates $(\mathrm{X}, \mathrm{Y}$ and $\mathrm{Z})$ can be replaced on each axis with a $\mathrm{Z}$-score value. This provides an alternative method of data transformation and creates an ideal three-dimensional dataset for cluster analysis (i.e. equal consideration of coordinates). A Z-score value is defined as “...the distance (expressed in standard deviations) between an observation within a set and the mean of the set," (Koetje 1991: 189). Through this process, an entirely new set of coordinates are created for each observation (artefact) in the sample. The use of the $Z$ score successfully transforms the entire data distribution into a standard space, where each axis has a coordinate mean of 0 (Koetje 1993: 118, Koetje 1992: 179). SPSS v.10 was used to calculate the standardized 
$\mathrm{Z}$ scores for the $\mathrm{X}, \mathrm{Y}$ and $\mathrm{Z}$ coordinates. Once calculated, the $\mathrm{Z}$ score values were entered in the database as separate columns and used for the k-means cluster analysis.

\subsubsection{3 - Combined Z Expansion Factor and Data Standardization Trial}

A third trial combined both the $\mathrm{Z}$ axis expansion factors and the $\mathrm{Z}$ score transformation, resulting in a standardized dataset with expansion factors applied. Once the results of the $\mathrm{Z}$ expansion factor trials are evaluated, the most suitable factor is selected and applied to the combined transformation trial. Results of the combined trial should improve the overall distribution of clusters by placing a strong emphasis on the $Z$ coordinates. Once complete, each of the transformation processes is compared to determine the most effective procedure for the identification of significant clustering in three dimensions.

\subsubsection{4 - Transformation Results}

The results of the transformation trials indicate that a $\mathrm{Z}$ expansion factor of 10 creates a suitable three-dimensional dataset for k-means cluster analysis. As discussed above, expansion factors of $5,10,15$ and 20 were applied to the $\mathrm{Z}$ coordinates of each level to improve the weighting of the $\mathrm{Z}$ axis in the cluster analysis. Table 5.2 below displays the original coordinate scale ranges for $\mathrm{X}, \mathrm{Y}$ and $\mathrm{Z}$, including the transformed ranges from the application of various expansion factors for archaeological levels II/2, III and IV. 


\begin{tabular}{|c|c|c|c|}
\hline 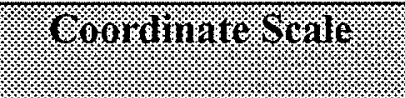 & 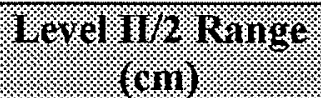 & 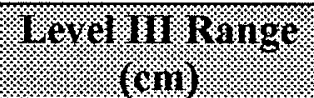 & 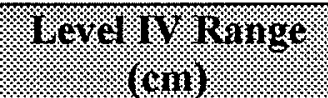 \\
\hline X Coordinate Scale & $3-596=593$ & $25-596=571$ & $5-597=592$ \\
\hline Y Coordinate Scale & $3-495=492$ & $5-480=475$ & $1-496=495$ \\
\hline Z Coordinate Scale & $1-57=56$ & $20-66=46$ & $11-77=66$ \\
\hline Z Expansion Factor 5 & $5-285=280$ & $100-330=230$ & $55-385=330$ \\
\hline Z Expansion Factor 10 & $10-570=560$ & $200-660=460$ & $110-770=660$ \\
\hline Z Expansion Factor 15 & $15-855=840$ & $300-990=690$ & $165-1155=990$ \\
\hline Z Expansion Factor 20 & $20-1140=1120$ & $400-1320=920$ & $220-1540=1320$ \\
\hline
\end{tabular}

Table 5.2: Coordinate Scale Ranges ( $\min$ to max depth below datum) with Applied Z Expansion Factors

As the table demonstrates, an expansion factor of 10 improves the three dimensional distribution of the dataset for each level, including the vertical distribution of points, while transforming the original $\mathrm{Z}$ coordinate scale into a comparable distribution range with the $\mathrm{X}$ and $\mathrm{Y}$ coordinates in each of the three levels. For example, level II/2 has an original $Z$ scale range of $1 \mathrm{~cm}$ to $57 \mathrm{~cm}$, which is very small when compared to the $X$ and $\mathrm{Y}$ coordinates. A Z expansion factor of 10 creates a distribution of $10 \mathrm{~cm}$ to $560 \mathrm{~cm}$, or $560 \mathrm{~cm}$ total, which places it within an acceptable range when compared to the $X$ and Y coordinates. The other expansion factors either exceeded or did not meet the required range, as indicated by the results in Table 5.2 (above).

It is equally important to consider the possibility of human error when recording artefacts in three dimensions. The artefacts recovered from Karabi Tamchin were recorded vertically by transit readings, which have an associated margin of error. When the vertical scale is expanded using $\mathrm{Z}$ expansion factors, the resulting margin of error is also increased by the same factor. The results of the comparison between each of the four $\mathrm{Z}$ expansion factor trials suggest that a factor of 10 is the most appropriate choice based on the resulting vertical distribution range and the visible improvement in clustering without horizontal banding. 
A $Z$ expansion factor 10 and the standardized $\mathrm{z}$ score trials were identified as the most appropriate and effective solutions for the transformation of the Karabi Tamchin dataset prior to k-means analysis. Both trials produced almost exactly the same results for the datasets of each level, indicating that the two transformations had similar influence and effectiveness on the k-means cluster analysis. Given the similarities between the results, a $Z$ expansion factor of 10 was selected to be applied to the datasets for each level for the three-dimensional analysis and interpretation of spatial organization. The $Z$ expansion factor 10 results will provide better visualization of the data and ease of interpretation in three dimensions, as it exaggerates the vertical scale, rendering the distribution of clusters more distinct.

\section{7 - Significance Testing}

The k-means program in Kintigh's TQA (1994) generates a computer printout of cluster memberships that can be used to identify individual artefacts within each cluster in the solution. A separate database file is created for each significant cluster solution for the archaeologically defined levels. Within the database, the cluster assignment for each artefact/point is recorded for future spatial analysis. The identification of cluster memberships allows the researcher to query the database by cluster number, making it possible to examine the attributes of points within each cluster. The cluster membership databases also contain all of the original attribute information including the $\mathrm{X}, \mathrm{Y}$ and $\mathrm{Z}$ coordinates, artefact type, level and square designation. These databases are converted into shapefiles and analyzed using ArcView (horizontal distribution) and ArcGIS (vertical distribution) to determine the $\mathrm{X}, \mathrm{Y}$ and $\mathrm{Z}$ value distribution of each cluster. The 
results are then used to evaluate the statistical significance of cluster contents for interpretation of the horizontal (intra-level) and vertical (intra-site) distribution of archaeological materials.

The k-means program provides important information regarding the distribution of materials and makes identification of spatially defined activity areas possible within each level. This requires a close examination of the artefacts within each of the clusters defined for a particular solution. The statistical significance of each cluster composition must be evaluated to determine whether the artefacts are randomly distributed throughout the site, or significantly distributed within specific clusters.

The Chi-square, or Goodness-of-Fit Test is used to compare "...the observed frequency counts of a single variable with an expected distribution of frequency counts allocated over the same categories" (McGrew and Monroe 2000: 155). The Chi-square test makes it possible to determine whether the contents of individual clusters in a given solution are significantly different. The Chi-square test requires that the input data must be actual frequency counts such as nominal or ordinal data. The test cannot be performed on ratio or interval data, such as percentages (McGrew and Monroe 2000: 155). Since each artefact or point within a given cluster is identified nominally by its corresponding $\mathrm{X}, \mathrm{Y}$ and $\mathrm{Z}$ coordinate, the Chi-square test is a suitable choice for the present dataset.

A standard Chi-square test requires a $2 \times 2$ contingency table. Given that the number of categories in this research is greater than two in all cases, a two by two contingency table is not appropriate. It is possible to create a contingency table based on more than two categories, called the " $\mathrm{rx} \mathrm{c}$ " contingency table, where $\mathrm{r}>2$ or $\mathrm{c}>2$ (Thomas 1986: 277). The test works on the same principles as the two-by-two Chi-square 
contingency table, although there are some important additional considerations. Thomas (1986: 298) states that the data must meet one of the following requirements when using the Chi-square $\mathrm{r} x \mathrm{c}$ Contingency table:

a. All expected values are greater than 5 ; or

b. No more than about 20 percent of the cells have expected values less than 5 and no expected value is less than 1 ; or

c. More than about 20 percent of the cells have expected values less than 5 and no expected value is less than 2 .

In order to test the significance of differences between individual clusters in each cluster solution, a separate contingency table is constructed using cluster pairs. Each cluster was paired together with all other clusters in the solution; however, similar pairings (for example, cluster 1 and 4 , cluster 4 and 1) were only run once, as they would produce the same results. Those pairs that did not meet the $\mathrm{r} x \mathrm{c}$ contingency table requirements outlined by Thomas (1986) were run for comparison, but the results will not be interpreted. In addition to the k-means cluster analysis, Kintigh's Tools for Quantitative Archaeology (1994) offers several statistical programs, including the TwoWay program for the analysis of two-dimensional contingency tables. Once the observed artefact frequencies for two clusters are entered, the Two-Way program calculates expected frequencies and the resulting Chi-square value. The program also produces a printout of the observed and expected tables, with a breakdown of the calculations. This provides a simple method for significance testing, with results that are easy to interpret. The Two-Way program was used in this analysis. The resulting Chi-square values are compared with the critical values for the appropriate degrees of freedom (results are presented in chapter 6$)$. 
The null hypothesis $\left(\mathrm{H}_{0}\right)$ developed for the Chi-Square test states:

There is no significance difference between the artefact compositions of any two clusters; the distribution of artefacts is based on a random distribution.

A standard level of .05 significance was selected to compare the resulting chisquare value with the critical value. If the null hypothesis is rejected, it may indicate cultural/spatial patterning within the level. Once this is determined, it is possible to make inferences about the spatial location and nature of activities that took place in each level. Rejection of the null hypothesis may also reveal important information regarding the use of space by pre-modern hominids, as discussed in chapter 7 .

\section{8 - Conclusions}

This chapter has presented a detailed discussion of the methodology developed to test the integrity of archaeological levels identified during excavation, to determine whether the horizontal clustering of artefacts can be used to distinguish activity areas within archaeologically defined levels. To summarize, initial investigations of the dataset indicate that it provides a random, representative sample of archaeological materials for cluster analysis. The k-means analysis program in SPSS v. 10 is then used to evaluate the total dataset distribution as a test of the integrity of the archaeologically defined levels in three dimensions.

Subsequent dataset preparation, including re-sampling and the application of a Z scale expansion factor of 10 , have created a three-dimensional dataset that is suitable for intra-level k-means cluster analysis using Kintigh's (1994) Tools for Quantitative Archaeology and visualized using GIS. The results are investigated statistically to 
identify significant differences between the contents of individual clusters. The horizontal distribution of artefact clusters is examined in chapters 6 and 7 based on the results of the significance tests to determine whether or not discrete spatial clusters can represent spatially discrete activity areas. This will allow the researcher to evaluate the effectiveness of the use of $k$-means cluster analysis combined with GIS as method for the analysis of three-dimensional spatial patterning within archaeologically defined levels at Karabi Tamchin. 


\section{- Chapter Six - Results}

\section{1 - Introduction}

This chapter will provide a detailed description of the results obtained from the three-dimensional K-means cluster analysis of Karabi Tamchin. The results of the SPSS trial are presented, followed by the individual level results from Kintigh's K-means program. The results of the significance testing will be described; clusters whose contents are statistically significant are also identified. The results will be interpreted in chapter 7 .

\section{2 - K-Means Cluster Analysis SPSS 10}

As described in chapter 5, SPSS v. 10 requires the user to specify the number of clusters for the analysis. The three dimensional distribution of points were visually inspected in three dimensions using ArcGIS to identify subjective clusters in the point distribution. Four distinct clusters with a potential fifth, less distinct cluster were identified.

A histogram plot of the $\mathrm{Z}$ distribution was also created for the purpose of objectively locating significant peaks (possible clusters) in the dataset. The histogram

(Figure 6.1) displays four strong peaks and a potential $5^{\text {th }}$ peak. The black arrows indicate strong peaks, while the striped arrow shows the possible location of a fifth peak. The results of the three-dimensional visual interpretation and the histogram indicate that the K-means cluster analysis should be run specifying 4 clusters. 


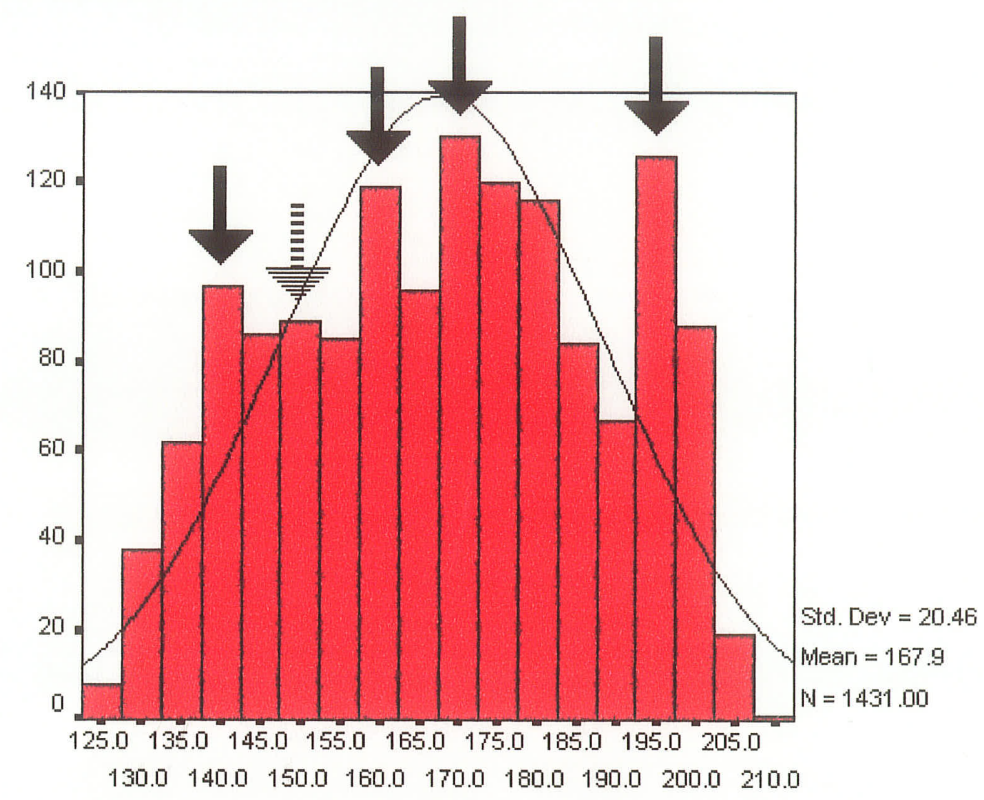

Z

Figure 6.1: Histogram Plot of Z-Value Distribution for All Levels: Arrows represent potential clusters in the dataset

Following the K-means cluster analysis, four clusters are defined and the cluster memberships are saved in the database. A point shapefile is created in ArcView containing all of the original information in the database $(\mathrm{X}, \mathrm{Y}$ and $\mathrm{Z}$ coordinates, artefact type, level and square) as well as the cluster membership for each point. The attribute table is queried to determine the vertical range for each of the 4 clusters, allowing each cluster to be evaluated separately. Table 6.1 (below), displays the vertical distribution values for each of the four K-means clusters:

\begin{tabular}{|l|l|l|l|}
\hline Cluster Number & \multicolumn{1}{|c|}{$\begin{array}{c}\text { Minimum Z } \\
\text { Value }\end{array}$} & \multicolumn{1}{|c|}{$\begin{array}{c}\text { Maximum Z } \\
\text { Value }\end{array}$} & Mean Z Value \\
\hline 1 & $124 \mathrm{~cm}$ & $146 \mathrm{~cm}$ & $138 \mathrm{~cm}$ \\
\hline 2 & $147 \mathrm{~cm}$ & $164 \mathrm{~cm}$ & $156 \mathrm{~cm}$ \\
\hline 3 & $165 \mathrm{~cm}$ & $184 \mathrm{~cm}$ & $174 \mathrm{~cm}$ \\
\hline 4 & $185 \mathrm{~cm}$ & $208 \mathrm{~cm}$ & $194 \mathrm{~cm}$ \\
\hline
\end{tabular}

Table 6.1: Z-Value Range for each Cluster in the SPSS v.10 K-Means Cluster Analysis 
I was also interested in the type and distribution of artefacts within each cluster for the identification of spatial trends. Each individual cluster is queried and selected points are converted into a separate shapefile. If the archaeological levels are spatially distinct, then the total artefact distribution should form clusters representative of each level (i.e. each cluster corresponds with 1 archaeological level). Cluster 1, 3 and 4 show strong associations with levels 2, IV and V/2, respectively. The majority of points in cluster 2 are identified as archaeological level III, however an almost equal number of points are from level II/2 (see below):

\section{Cluster $1=258$ points Level II 10 points Level III 3 points Level IV 1 point Level V/2}

$\begin{aligned} \text { Cluster } \mathbf{3}= & \mathbf{3 8} \text { points Level II } \\ & \text { 101 points Level III } \\ & \mathbf{2 8 0} \text { points Level IV } \\ & 44 \text { points Level V/2 }\end{aligned}$

\author{
Cluster 2 $=124$ points Level II \\ 146 points Level III \\ 58 points Level IV \\ 16 points Level V/2
}
Cluster 4 = No Level II 11 points Level III 93 points Level IV 248 points Level $V / 2$

To view the clusters in 3 dimensions, each cluster shapefile was opened in ArcMap (ArcGIS) and viewed using the Trend Analysis Geostatistical extension. This also provides a means of comparing the clusters identified by the K-means program with the actual archaeologically defined levels as displayed in the two-dimensional western wall profile. The following figures (6.2a, b, c and d) display the three-dimensional point distribution for each cluster. 


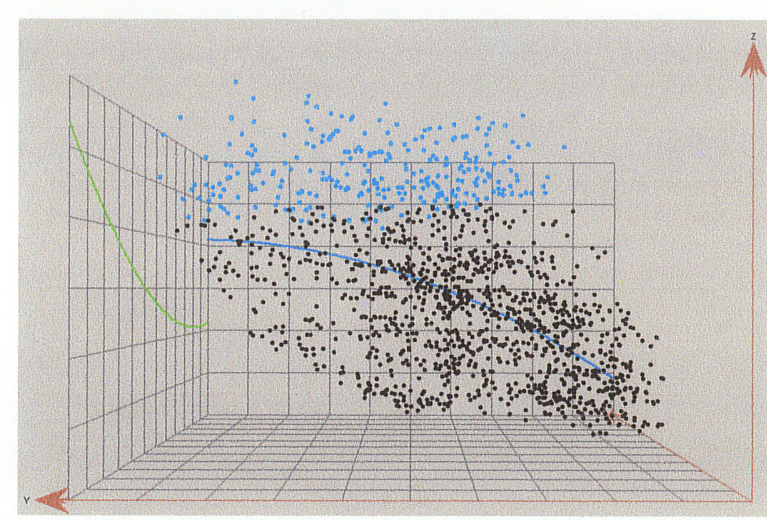

6.2a: Cluster 1

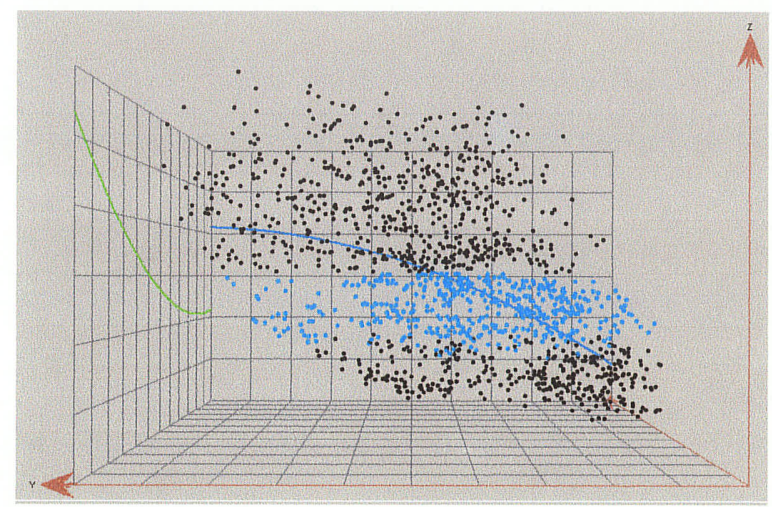

6.2c: Cluster 3

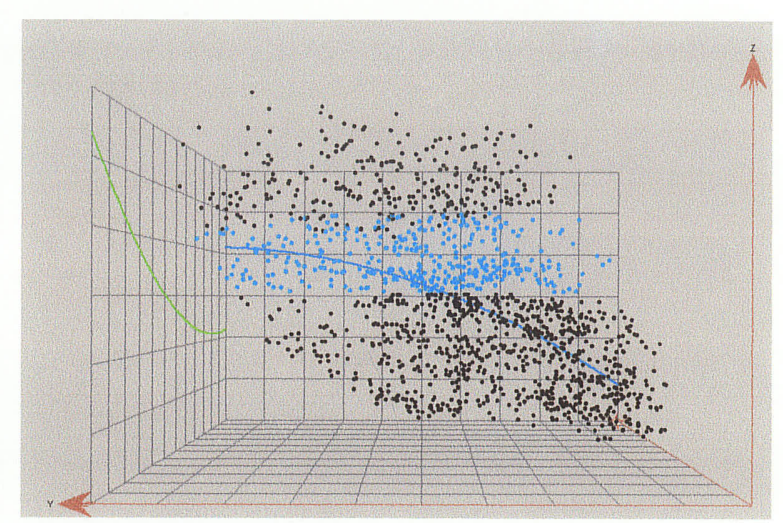

6.2b: Cluster 2

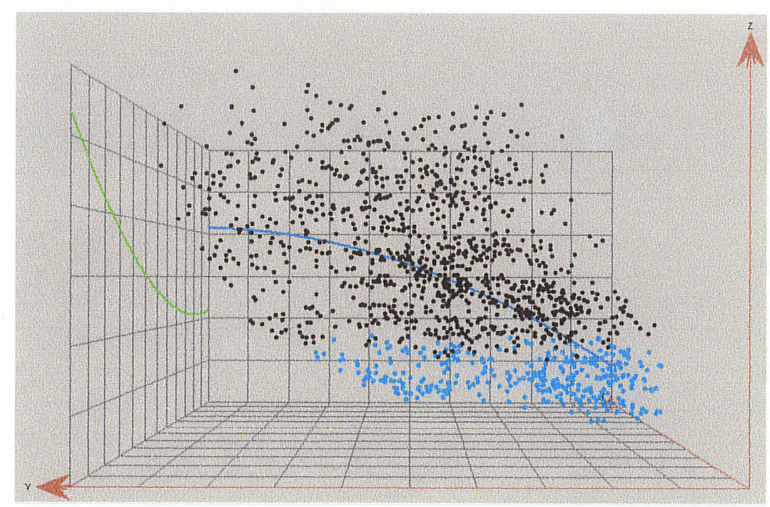

6.2d: Cluster 4

Figure 6.2a, b, c and d: Final Clustering Results for the 4-Cluster K-means Analysis

The cluster centre or mean, is also compared with the $\mathrm{Z}$ values of the histogram peaks to determine whether there were any similarities (see Table 6.1 and Figure 6.1). Initial observations suggest that the SPSS v. 10 trial was successful in identifying meaningful clusters, which correspond with archaeological levels II/2, III, IV and V/2

\section{3 - Kintigh's Tools for Quantitative Archaeology Level by Level Analysis}

Once the K-means analysis was performed using Kintigh's program, a computergenerated graph of the Sum Squared Error (SSE) was produced and used to determine the most significant clustering solutions, ranging from 1 to 20 clusters. Inflection points, or 
elbows along the curve were used to identify drops in the SSE, or non-random clustering solutions (Koetje 1989: 56). Within each of the three archaeological levels examined here, the clustering solutions identified at each inflection point along the SSE plot were visually examined; the spatial distribution and contents of each cluster will be described below. The k-means cluster analysis of the sampled datasets for levels $\Pi / 2, \Pi$ and 4 was performed using a $\mathrm{Z}$ expansion factor of 10 . Clustering results were subjected to a Chisquare significance test of individual cluster contents. The results of the cluster analysis and subsequent statistical testing are presented below for each of the 3 archaeological levels.

\subsection{1 - Level $\Pi / 2$}

The K-means SSE plot for the level II/2 dataset identified significant clustering solutions at 3 and 8 clusters (Figure 6.3 below). Cluster solutions can be viewed in a three-dimensional spinning plot, however the images cannot be presented here. Instead, a series of horizontal, two-dimensional plots will be presented (see Appendix C). The 3cluster solution creates three relatively large clusters of approximately $9 \mathrm{~m}^{2}$, which are spatially distinct (Appendix C - Figure 1). Cluster 3-1 is located in the western section of the level and contains a total of 68 artefacts within the southwest edge of the excavated level. Cluster 3-2 contains 70 artefacts and is located in the north-central section of the level, with materials dispersed towards the centre. Cluster 3-3 occupies the front (southern portion) of the site and contains 43 artefacts. Cluster 3-3 is also more dispersed. 


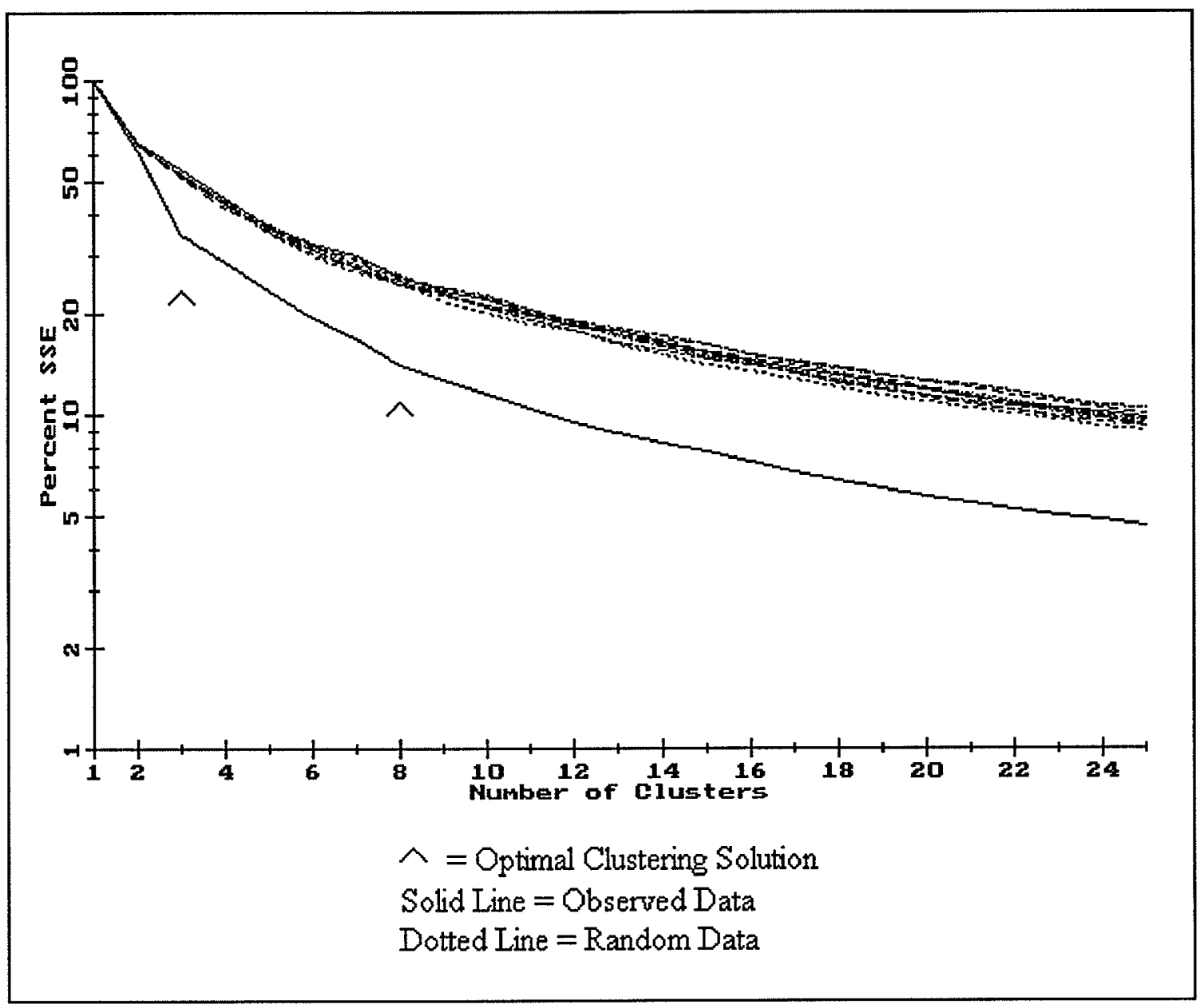

Figure 6.3: Sum Squared Error Graph for Level II/2

The 8-cluster solution creates a series of smaller clusters ranging in size from approximately $2 \mathrm{~m}^{2}$ to $4 \mathrm{~m}^{2}$, which demonstrate some degree of overlap at various locations throughout the level (Appendix C - Figure 2). Cluster 8-1 is one of the larger clusters, approximately $3 \mathrm{~m}^{2}$ and contains 35 artefacts. It is located in the southwest section of the shelter and overlaps considerably with cluster 8-8. An examination of the distribution of artefacts from cluster 8-1 and 8-8 suggests that there is significant overlap between the two, which may provide evidence of palimpsests when viewed three dimensionally (discussed in Chapter 7). Cluster 8-2 is located in the northwest, along the 
northern perimeter of the site and contains 31 artefacts. Cluster $8-2$ is generally spatially distinct, however, there is slight overlap with cluster 8-7. Cluster 8-3 contains 17 artefacts and occupies the centre of the southern half of the level. Cluster 8-4 is another large cluster of 35 artefacts occupying approximately $4 \mathrm{~m}^{2}$ in the eastern section of the level. Cluster 8-4 demonstrates some overlap with cluster 8-7. Cluster 8-5 is located in the southeast, with a total of 16 artefacts. The materials demonstrate a slight overlap with cluster 8-4. Cluster 8-6 is a very small, spatially distinct cluster of 4 artefacts with an approximate diameter of $2 \mathrm{~m}^{2}$. Given the size of this cluster, it will be very difficult to interpret the materials in terms of spatial patterning at the potential location of cultural activities. However, the identification of these materials as a separate cluster in the kmeans analysis suggests that there may be an important three-dimensional distinction that will reveal information concerning the nature of occupation within this level (chapter 7). Cluster 8-7 occupies the northernmost section of the site, with concentrations along the northern perimeter. A total of 27 concentrated artefacts are identified as cluster 8-7, which displays some overlap with cluster 4 . Finally, cluster 8-8 is a smaller cluster of 16 artefacts occupying approximately $2 \mathrm{~m}^{2}$ the western perimeter of the level. As mentioned above, this cluster overlaps cluster 8-1. This relationship will be closely examined in three dimensions and the resulting interpretation will be presented (chapter 7).

\subsubsection{1 - Significance Testing}

Significance tests were performed for pairs of clusters in the 3 and 8 cluster solutions for level II/2. Within the 3-cluster solution, none of the cluster pairs were able to reject the null hypothesis at the .05 level of significance (Appendix D - Tables 10-12). 
These results suggest that the contents of each cluster are comparable and represent a random distribution of find types throughout the site. While the clusters themselves may be non-randomly distributed, their contents do not permit us to differentiate between individual clusters.

As outlined in chapter 5, the application of a Chi-square contingency table requires that the expected values follow a series of recommended criteria. Unfortunately, the distribution of artefacts in the 8-cluster solution created very small clusters (Cluster 86) and the requirements could not be met for all cluster pairs in the solution. The results of cluster pairs 6 (8-1 and 8-7), 7 (8-1 and 8-8), 12 (8-2 and 8-7), 13 (8-2 and 8-8) and 28 (clusters 8-7 and 8-8) also failed to meet the requirements of the chi-square contingency table.

A total of 28 cluster pairs were analyzed statistically for the 8-cluster solution (Appendix D - Tables 13-40). Cluster pairs 3 (clusters 8-1 and 8-4), 8 (clusters 8-2 and 83), 17 (clusters 8-3 and 8-7) rejected the null hypothesis at the .05 significance level. The remaining cluster pairs did not reject the null hypothesis. These results suggest that the contents of individual clusters within the 8-cluster solution for level $\mathrm{I} / 2$ dataset are not significantly different and artefacts are randomly distributed within clusters.

\subsection{2 - Level III}

The application of an expansion factor of 10 was applied to the $\mathrm{Z}$ coordinates to improve the formation of clusters in three dimensions. The resulting SSE plot identified optimal clustering solutions at 2, 4 and 6 clusters (Figure 6.4 below). The 2-cluster solution divides the site into two distinct concentrations; cluster 2-1 occupying the 
Southeast half of the site and cluster II/2 situated in the Northwest half of the shelter (Appendix C - Figure 3). Cluster 2-1 contains 122 artefacts, which are generally concentrated along the eastern edge of the excavation. Cluster 2-2 is dispersed across the back of the shelter, containing a total of 126 artefacts.

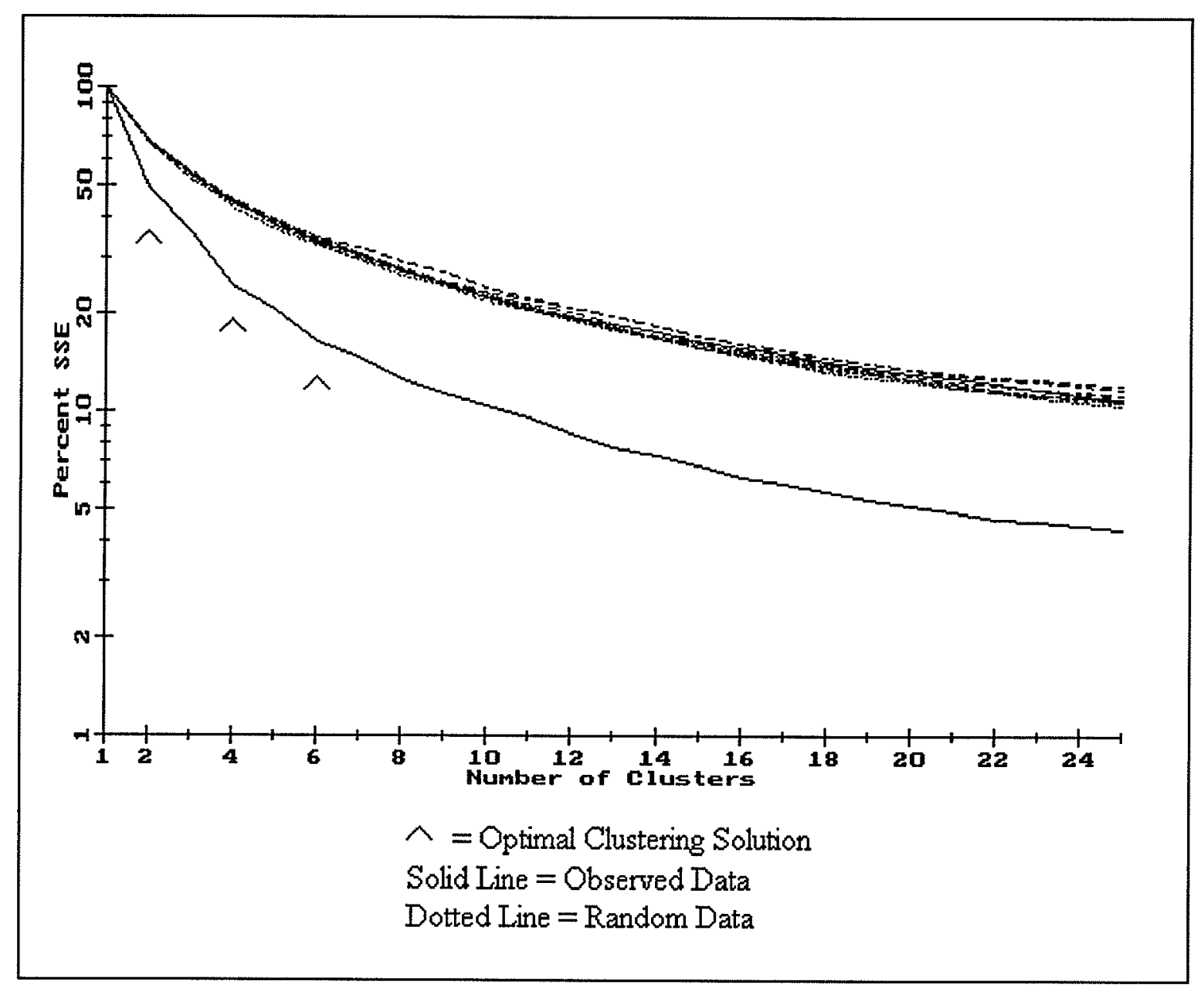

Figure 6.4: Sum Squared Error Graph for Level III

The 4-cluster solution creates four clusters of approximately the same size in diameter (Appendix C - Figure 4). Cluster 4-1 is situated in the eastern section of the site and contains 66 artefacts. Cluster 4-1 also demonstrates a slight overlap of materials with 
cluster 4-4. Cluster 4-2 contains 59 artefacts, which demonstrate slight concentration in the northern section of the excavated level. A total of 60 artefacts are identified as cluster 4-3, located in the western section of the level towards the centre. Cluster 4-4 contains 63 artefacts, which are concentrated in the southeast corner of the excavation perimeter. The vertical distribution as well as the contents of each cluster will be examined in chapter 7 to determine whether the association of artefacts within clusters is significant for the identification of activity areas.

Within the 6-cluster solution, clusters 1-5 are approximately the same size in diameter (approximately $4 \mathrm{~m}^{2}$ ), while cluster 6 is comparatively smaller occupying approximately $2 \mathrm{~m}^{2}$ of the excavated level (Appendix C - Figure 5). Cluster 6-1 contains 45 artefacts and is concentrated along the eastern perimeter towards the north. Cluster 6-2 occupies the very back of the cave, (northern section), with 54 artefacts. Cluster 6-2 also demonstrates a slight overlap with clusters 6-1 and 6-3 towards the centre of the level. Cluster 6-3 is located in the very centre of the site with 41 artefacts, surrounded by the other five clusters. There is also considerable overlap between cluster 6-3 and 6-5. Cluster 6-4 is concentrated in the southeast corner of the site, consisting of 48 artefacts. Cluster 6-5 contains 40 artefacts in the west. As mentioned above, there is also a noticeable overlap of materials between cluster 6-3 and 6-5. Finally, cluster 6-6 is located along the south perimeter, consisting of 20 artefacts. The distribution of cluster 6-6 does not demonstrate any overlap with the existing clusters in the solution. 


\subsubsection{1 - Significance Testing}

Significance tests were performed for pairs of clusters in the 2,4 and 6 cluster solutions for level III (Appendix D - Tables 41-62). The results of the Chi-square contingency table for the 2-cluster solution rejected the null hypothesis at the required .05 level of significance, indicating that the contents of each cluster are significantly different.

The 4-cluster solution for the level III dataset produced interesting statistical results (Appendix D - Tables 42-47). Cluster pairs 3 (cluster 4-1 and 4-4), 4 (4-2 and 4-3) 5 (4-2 and 4-4) and 6 (4-3 and 4-4) rejected the null hypothesis at the .05 level of significance, indicating that the contents of these clusters are significantly different and can be interpreted for the possible identification of activity areas.

The 6-cluster solution creates a series of 15 cluster pairs, which were analyzed using a Chi-square contingency table (Appendix D - Tables 48 to 62). Cluster pair 14 (clusters 6-4 and 6-6) failed to met the requirements for minimum cluster contents outlined in the methods chapter, as more than $20 \%$ of the expected values are less than 5 and one of the expected values is less than 2 . Therefore, the result of the chi-square test for cluster pair 14 is not considered. Cluster pairs 1 (cluster 6-1 and 6-2), 4 (6-1 and 6-5), 6 (6-2 and 6-3), and 13 (6-4 and 6-5) did not reject the null hypothesis when tested at the .05 significance level. The remaining cluster pairs $(2,3,5,7-12$ and 15$)$ rejected the null hypothesis at the .05 level of significance. These results of the chi-square test for level III suggest that it may be possible to interpret the contents of individual clusters in relation to the organization of activity areas. It will also be necessary to examine the threedimensional distribution of clusters to determine whether those clusters with comparable 
contents demonstrate differential vertical distribution and are representative of different occupational phases.

\subsection{3 - Level IV}

The K-means SSE plot identified optimal clustering solutions at 3 and 5 clusters (Figure 6.5 below). The results of this trial will be presented below and the interpretation of cluster contents will be discussed in chapter 7. The 3-cluster solution divides the site into three large sections, with clusters in the north, southeast and southwest sections of the level (Appendix C - Figure 6). Cluster 3-1 contains 84 artefacts and occupies the southeast section of the level towards the centre of the site. Cluster 3-1 also exhibits some overlap with cluster 3-3 towards the northern section of the level. Cluster 3-2 consists of 92 artefacts in the western section of the level with a diameter of approximately $4 \mathrm{~m}^{2}$. There is a very small overlap of materials between cluster 3-2 and 3-3 within the centre of the level in the northern half of the level. Cluster 3-3 is the smallest cluster, with a total of 63 artefacts occupying approximately $6 \mathrm{~m}^{2}$ in the northern half of the level.

The 5-cluster solution creates a series of clusters that range from approximately $2 \mathrm{~m}^{2}$ to $6 \mathrm{~m}^{2}$ in diameter and exhibit varying degrees of overlap between individual clusters (Appendix $\mathrm{C}-$ Figure 7). Cluster 5-1 contains 42 artefacts located along the eastern perimeter of the level. Cluster 5-1 also overlaps considerably with cluster 5-2 in the southeast corner of the site. Cluster 5-2 consists of 32 artefacts located in the south, towards the centre of the level. The distribution of cluster 5-2 materials is generally spatially distinct however, there is distinct overlap of materials with cluster 5-1 in the southeast corner. A total of 58 artefacts are identified as cluster 5-3, which is located 
within the centre of the level. Cluster 5-4 is a comparatively small $\left(3 \mathrm{~m}^{2}\right)$ cluster of 35 artefacts located along the northern perimeter of the site. There is also a slight overlap of materials between cluster 5-3 and 5-4 towards the centre of the site. Finally, Cluster 5-5 contains a large cluster of 72 artefacts in the western section of the level.

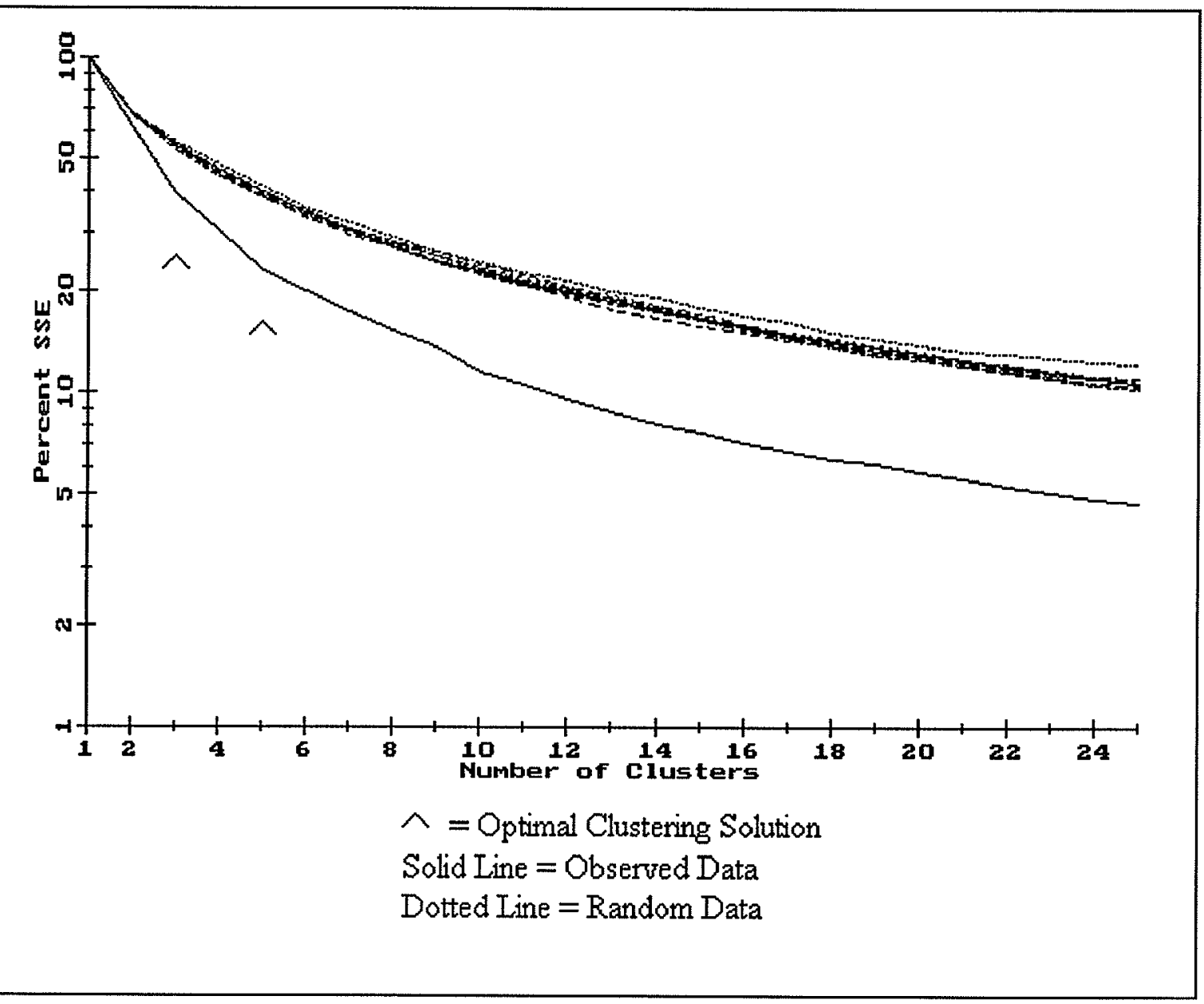

Figure 6.5: Sum Squared Error Graph for Level IV 


\subsubsection{1 - Significance Tests}

Chi-square contingency tables were produced for cluster pairs in the 3 and 5 cluster solutions for the level IV dataset. The results of the 3 cluster solution chi-square tests revealed interesting information concerning the distribution of materials in level IV (Appendix D - Tables 63-65). None of the cluster pairs were able to reject the null hypothesis at the .05 level of significance, indicating that the cluster contents of each cluster in the solution are comparable.

The 5-cluster solution created a total of 10 cluster pairs that were statistically tested using the chi-square contingency table (Appendix D - Tables 66-75). Cluster pair 3 (cluster 5-1 and 5-4) rejected the null hypothesis at .05 significance level, however the remaining cluster pairs did not. The results of the 3 and 5 cluster solution chi-square tests may suggest that activities within the shelter were not spatially distinct. As discussed above, the distribution of clusters also demonstrated varying degrees of overlapping; therefore, an examination of each cluster in three dimensions may reveal differences in the vertical distribution of materials, which are indicative of a palimpsest of archaeological materials.

\section{4 - Conclusions}

The results of the K-means cluster analysis for the SPSS 10 trial and the individual level analysis using Kintigh's Tools for Quantitative Archaeology (1994) have been presented and described. Chapter 7 provides an interpretation and discussion of the results and evaluates the suitability of the $\mathrm{K}$-means clustering program for manipulating three-dimensional data, while providing a means of statistically testing the accuracy of 
the archaeologically defined levels. Distinct clusters of finds can be identified in all 3 archaeological levels. Cluster contents prove significantly different in level III only and will be interpreted in terms of activity areas.

Chapter 7 provides a detailed discussion of the clustering results for the sampled datasets of levels II/2, III and IV. The optimal clustering results of the SSE plots will be interpreted in three dimensions with the assistance of the ArcGIS trend analysis function. The horizontal and vertical location of clusters will be examined for the possible identification of palimpsests. In addition, the artefact contents of individual clusters will be discussed in combination with the results of the cluster pair chi-square statistical tests and the spatial distribution of faunal materials and burnt bone materials. Cluster pairs that rejected the null hypothesis will be interpreted with respect to spatial organization and the potential location of distinct activity areas within each level. Cluster pairs that did not reject the null hypothesis will only be evaluated in terms of interpretations of their vertical distribution in relation to site topography. 


\section{- Chapter Seven - \\ Discussion and Interpretation}

\section{1 - Introduction}

The results of the three-dimensional K-means analysis were presented in chapter six. It is now necessary to interpret the clustering results and statistical significance testing for the sampled datasets. This will be done using the three-dimensional graphical capabilities of GIS, which will provide a means of interpreting and discussing the spatial organization of each level as well as the artefact association within optimal cluster solutions for the Karabi Tamchin dataset. It also provides an opportunity to evaluate the effectiveness and suitability of GIS, combined with quantitative analysis, for the interpretation of three-dimensional datasets.

The results of the SPSS v. 10 trial will be discussed and compared with the archaeologically defined stratigraphic profile. Next, results of the K-means analysis will be discussed. The SPSS v.10 K-means analysis identified 4 clusters in the dataset with distinct vertical clustering, confirming the presence and location of four distinct cultural levels. In chapter 5, it was determined that archaeological level V/2 and sub-level 2-1 would not be suitable for K-means cluster analysis because insufficient materials had been recorded in three-dimensions across the level. The following discussion will examine the spatial distribution of optimal cluster solutions identified by the k-means cluster analysis of levels II/2, III and IV using a Z expansion factor of 10 . Each of the three archaeologically defined levels investigated will be examined individually and will be interpreted to determine whether it is possible to establish behavioural and cultural interpretations for successive occupation levels at Karabi Tamchin (for example, the location of hearths and chipping stations within the site). 
Modern thinking regards the archaeological site as a place that was exploited by people of the past; therefore, it is possible to use variability in the spatial distribution of finds to interpret the differential organization of space and the location of unique activity areas (Binford 1996: 229). The archaeological materials recovered from Karabi Tamchin suggest that tool use and manufacture were activities carried out on site, as well as the processing of animal remains for consumption. Therefore, it is possible that the distribution and contents of individual clusters will indicate the potential location of these activities.

This thesis also aims to develop an innovative method of analysis for the interpretation of palimpsests, which are present in many prehistoric deposits especially where small, mobile groups are present (Spikins et al. 2002: 1235). If there are palimpsests present at Karabi Tamchin, individual clusters will demonstrate vertical overlap when viewed in three dimensions, indicating sediment accumulation between two or more distinct occupation phases. Furthermore, if site function does not vary, or the organization of space within the site stays the same over time, the contents of palimpsest clusters should also be comparable, as they represent a distinct period of occupation, rather than a specific activity area within a larger spatial occupation (Simek et al. 1985: 231). Specific activity locations within the occupation area should demonstrate differences in artefact composition. The possible identification of within-level palimpsests will contribute to a better understanding of the types of activities and occupations at Karabi Tamchin and the results will be used to expand the current understanding of regional occupations of the Crimea throughout the Middle Palaeolithic. 


\section{2 - SPSS v. 10 Analysis}

The results of the SPSS v. $10 \mathrm{~K}$-means analysis are now compared with the stratigraphic profile. If the archaeologically defined levels have been correctly identified, there should be a strong correspondence between the vertical range of the archaeological levels and the range of the K-means clusters. Table 7.1, below, displays the $Z$ Value range for each of the four archaeologically defined levels and for the four clusters:

\begin{tabular}{|c|c|c|c|}
\hline I level] & (I) Vax & $\begin{array}{l}\text { SPSS } \\
\text { " Clinster. }\end{array}$ & Max Valuir: Min \\
\hline II & $124 \mathrm{~cm}-180 \mathrm{~cm}$ & 1 & $125 \mathrm{~cm}-146 \mathrm{~cm}$ \\
\hline III & $143 \mathrm{~cm}-189 \mathrm{~cm}$ & 2 & $147 \mathrm{~cm}-164 \mathrm{~cm}$ \\
\hline IV & $134 \mathrm{~cm}-200 \mathrm{~cm}$ & 3 & $164 \mathrm{~cm}-184 \mathrm{~cm}$ \\
\hline $\mathrm{V} / 2$ & $146 \mathrm{~cm}-208 \mathrm{~cm}$ & 4 & $185 \mathrm{~cm}-188 \mathrm{~cm}$ \\
\hline
\end{tabular}

Table 7.1: Z-Value Range for the Archaeologically Defined Levels and SPSS Clusters

ArcView GIS is used to extract points from squares adjacent to the profile and calculate the $\mathrm{Z}$ value distributions for clusters, as the query function is useful for selecting specific attributes from a database. The stratigraphic profile (Figure 4.2) contains information for the western wall of the site for squares 8-I, 8-H, 8-G and 8-F. Stratigraphic profiles for the eastern, northern and southern walls are presently unavailable, but should be made available for future analysis.

Lithological and archaeological levels in the site are likely to vary in depth according to the topography of the rock shelter floor (and other topographic features such as large blocks, etc.). Therefore, a comparison is done along line 8 (square $\mathrm{F}$ to $\mathrm{I}$ ) between points in the SPSS v.10 k-means clusters and height values of threedimensionally recorded materials from each archaeological level, in order to compare clusters and stratigraphy. The attribute information for finds in squares 8-I, 8-H, 8-G and 8-F is isolated and used to produce height values for each archaeological level along the 
west profile, using the query function in ArcView. A new shapefile is created containing only points from line 8 and the corresponding attribute table. Through a comparison of the minimum and maximum $Z$ values of clusters along line 8 and the minimum and maximum $\mathrm{Z}$ values for points from the archaeologically defined levels, it is possible to establish whether the cluster program supports the identification of 4 distinct occupation levels and whether these correspond to the levels as defined during excavation.

\subsection{1 - SPSS v. 10 K-means Cluster 1}

Cluster 1 corresponds with lithological stratum 5, which contains archaeological level 2-1 and II/2. All of the points assigned to cluster 1 are identified as belonging to archaeological level 2 along row 8, indicating that there is a strong correlation between

cluster 1 and level 2. The archaeologists identified two sub levels in level 2: level 2-1 and II/2, which were identified in separate lithological strata. Archaeological level 2-1 is contained within lithological strata $5 \mathrm{a}$ and $5 \mathrm{~b}$, which have a vertical range of approximately $110 \mathrm{~cm}$ to $140 \mathrm{~cm}$. Level II/2 lies in lithostratum $5 \mathrm{c}(140 \mathrm{~cm}$ to $153 \mathrm{~cm})$. Some of the points in cluster 1 fall within the vertical range of strata $5 \mathrm{a}$ and $\mathrm{b}$, though they have been identified as belonging to archaeological sub-level level II/2. This finding may indicate that archaeological levels $2-1$ and II/2 should be classified together as one level. Alternatively, this could indicate that a lack of vertical separation (see discussion below) impedes the use of SPSS v.10 analysis as a means of identifying sub-levels. 


\subsection{2 - SPSS v. 10 K-means Cluster 2}

Cluster 2 does not correspond as well with archaeological level designations; it corresponds roughly to level III. Cluster 2 contains points from levels II/2, III, IV and V/2 (Chapter $6-$ p. 95). The vertical range for cluster 2 is approximately $147 \mathrm{~cm}$ to 164 $\mathrm{cm}$ with an average of $153 \mathrm{~cm}$. The west stratigraphic profile indicates that stratum $6 \mathrm{a}$, located at approximately $147 \mathrm{~cm}$ to $164 \mathrm{~cm}$, is associated with archaeological level III. The remaining points in cluster 2 fall into lithological stratum $5 \mathrm{c}$ (and are classified as belonging to archaeological sublevel level II/2) or stratum $6 \mathrm{~b}$ (and are classified as archaeological level IV). The vertical distribution of cluster 2 overlaps the vertical distribution of cluster 1 and cluster 3 , which explains this pattern. This suggests that either SPSS v. 10 is unable to clearly demarcate the archaeological levels because no expansion factor is used, meaning the resulting horizontal distributions are overshadowed by the effect of the vertical axis in very thin deposits, or that level III (associated with cluster 2) is ill-defined and may even be a palimpsest.

\subsection{3 - SPSS v. 10 K-means Cluster 3}

The points within cluster 3 are associated with archaeological levels IV and V/2. Cluster 3 has a vertical range of $164 \mathrm{~cm}$ to $184 \mathrm{~cm}$ with an average of $176 \mathrm{~cm}$. This corresponds well with the approximate range for archaeological level IV, which lies in the lower portion of lithological strata $6 \mathrm{~b}$ and $7 \mathrm{~b}$, although it also includes stratum $7 \mathrm{c}$ (archaeological level V/2). The points in cluster 3 that are identified as archaeological level V/2 have a vertical range of 172 to $184 \mathrm{~cm}$, which positions them within lithological stratum $7 \mathrm{c}(170$ to $189 \mathrm{~cm})$. As discussed in the results for cluster 4 (below), the close 
proximity of lithological strata $6 \mathrm{~b}, 7 \mathrm{~b}$ and $7 \mathrm{c}$ may have resulted in a mixture of points from levels IV and V/2 as there is no distinct stratigraphical separation between levels. Cluster 3 is not present within squares $8-\mathrm{G}$ and $8-\mathrm{F}$.

\subsection{4 - SPSS v. $10 \mathrm{~K}$-means Cluster 4}

Cluster 4 has a vertical range of $185 \mathrm{~cm}$ to $188 \mathrm{~cm}$, with an average of $186 \mathrm{~cm}$. This cluster displayed an affinity with archaeological level V/2 in lithological stratum 7c, which has a $Z$ value range of approximately $184 \mathrm{~cm}$ to $191 \mathrm{~cm}$. In addition, most of the points assigned to cluster 4 were identified as archaeological level V/2, (which lies in stratum $7 \mathrm{c}$ ). Not all level V/2 points are identified as cluster 4 , as several of them are assigned to cluster 3 (above) and some to cluster 2 . Lithological strata $6 \mathrm{~b}, 7 \mathrm{~b}$ and $7 \mathrm{c}$ were deposited as very thin layers and the SPSS v.10 K-means analysis may have identified some materials from archaeological level V/2 as cluster 3 due to the proximity of the lithological levels. Cluster 4 also contains points from archaeological level 4.

Lithological strata $6 \mathrm{~b}$ and $7 \mathrm{~b}$ are very thin strata containing level IV and stratum $7 \mathrm{~b}$ thins out and disappears halfway through the west profile (Chapter Four - Figure 4.2). Archaeologically defined levels IV and V/2 are positioned directly on top of one another on the west baulk, which may explain why they were grouped into the same cluster. Without the application of a $\mathrm{Z}$ expansion factor, the distinction between these strata would be extremely difficult, again demonstrating a potential weakness with the SPSS v. $10 \mathrm{~K}$-means cluster analysis. Cluster 4 is not present in squares $8-\mathrm{G}$ and $8-\mathrm{F}$. 


\subsection{5 - Conclusions}

A comparison of cluster locations, archaeologically defined levels and lithostratigraphic levels, using the stratigraphic profile, shows that the SPSS v. $10 \mathrm{~K}$ means analysis confirms the existence of four distinct cultural levels. The program identifies four artefact clusters that correspond fairly well with archaeologically defined levels. However, it is evident that very thin lithological strata interfere with the ability for the program to clearly distinguish vertical separations (see especially cluster 2 discussion above). This could be the result of a methodological limitation (i.e. no $Z$ expansion factor), which is an important consideration at Karabi Tamchin, where archaeological levels are not separated by thick sedimentary deposits and often lie directly on top of one another.

\section{3 - Kintigh's K-means Cluster Analysis}

\subsection{1 - Level II/2}

The K-means analysis (with a Z Expansion Factor of 10) identified optimal clustering solutions at 3 and 8 clusters for level II/2. These cluster solutions will now be interpreted, taking the results of the chi-square significance testing into consideration. In addition, the ArcGIS trend analyst function will be used to analyze individual clusters in three-dimensions.

Visual inspection of two-dimensional plans indicate that the horizontal distribution of artefacts throughout level II/2 is relatively even with a concentration of materials in the northwest (Appendix B - Figures 1-4). Faunal materials represent more than half of the total dataset concentrated along the northwest perimeter of the level. 
Stone flakes exhibit a similar distribution. The majority of stone tools are located in the centre of the level and a few are located in the outer edges of the site. The patterns observed with the naked eye could result from differential use of space, or they could be a result of random patterning. The $\mathrm{K}$-means program offers a means of testing whether or not finds are significantly patterned in their distribution. Once it is demonstrated that clusters exist, individual cluster contents can be examined and interpreted in terms of spatial activities within the level.

\subsubsection{1 - Level II/2: 3-Cluster Solution}

The 3 cluster solution consists of three relatively large clusters each containing bone, stone chips, stone flakes and stone tools. Using Arcview GIS, a two-dimensional point distribution of clusters was created for horizontal spatial interpretation (Appendix E

-Figure 1). Within the plans, artefacts were colour-coded according to cluster membership, making it possible to identify the spatial distribution of clusters in relation to each other. The clusters are spatially distinct, with very little overlap between individual clusters when viewed in two dimensions. The clusters are located in the west, the southeast or entrance of the shelter and in the back of the shelter.

Cluster 3-1 is located in the western section of the shelter; it contains 68 artefacts and is visually identifiable. Table 7.2 (below) displays the frequency and percentage distribution of artefact types within cluster 3-1. 


\begin{tabular}{|c|c|c|}
\hline Mrefart Woe & 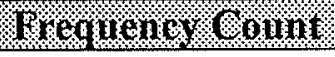 & Precentagerol 10042 \\
\hline Bone & 39 & 57.4 \\
\hline Chips and Flakes & 23 & 33.8 \\
\hline Stone Tools & 6 & 8.8 \\
\hline Total & 68 & 100.0 \\
\hline
\end{tabular}

Table 7.2: Cluster 3-1 Artefact Frequency and Percentage Distribution for the $Z$ Expansion Factor-10 3 Cluster Solution, Level II/2

Cluster 3-2 occupies the southeast section, and contains 70 artefacts. This cluster is distributed across a relatively wide area. Visual inspection of level II/2 did not reveal the presence of cluster 3-2. Table 7.3 (below) shows the frequency and percentage distribution of artefacts within cluster 3-2. Faunal materials represent approximately $64 \%$ of the total distribution, while stone flakes represent $21 \%$ and stone tools make up the final $14 \%$.

\begin{tabular}{|c|c|c|}
\hline Arutal Mor & Trequency ( voun & Mercentage or rotal \\
\hline Bone & 45 & 64.3 \\
\hline Chips and Flakes & 15 & 21.4 \\
\hline Stone Tools & 10 & 14.3 \\
\hline Total & 70 & 100.0 \\
\hline
\end{tabular}

Table 7.3: Cluster 3-2 Artefact Frequency and Percentage Distribution for the $\mathrm{Z}$ Expansion Factor-10 3 Cluster Solution, Level II/2

Cluster 3-3 is located in the northern section of the rock shelter with 43 artefacts near the back wall. Cluster 3-3 was also not readily identifiable upon visual inspection of the twodimensional plots. Cluster contents are listed in Table 7.4 (below):

\begin{tabular}{|c|c|c|}
\hline Mrtefact I Ype & Hrequerco Contil & Pevernageof Gotul \\
\hline Bone & 23 & 53.5 \\
\hline Stone Flakes & 10 & 23.2 \\
\hline Stone Tools & 10 & 23.3 \\
\hline Total & 43 & 100.0 \\
\hline
\end{tabular}

Table 7.4: Cluster 3-3 Artefact Frequency and Percentage Distribution for the $Z$ Expansion Factor-10 3 Cluster Solution, Level II/2 


\subsubsection{2 - Significance}

The two-way chi-square contingency table showed that none of the clusters in the 3-cluster solution is significantly different in content. There is no burnt bone in Level II $/ 2$, so the location of a fire pit or hearth cannot be established. The presence of carnivores within level $\Pi / 2$ is attested, including hyena and cave bear (Burke In Press). The relatively uniform distribution of archaeological materials could support the hypothesis that level II/2 was disturbed by carnivores. The cultural distribution of materials may have been disturbed over time, effectively distorting or destroying the original spatial distribution of artefacts within the level. Alternatively, as Binford (1996) suggests, there may be no cultural differentiation of space within the shelter.

When the contents of each cluster are compared through subjective analysis, it is clear that each has a comparable frequency and percentage of each artefact type. Cluster 3-2 has the highest percentage of bone and a lower percentage of stone tools and flakes (though the chi-square test shows that this is not significant). This may provide weak evidence for the suggestion that the back of the shelter was used for discarding materials, as the majority of faunal remains at the site would naturally accumulate in these areas because of food processing, consumption and refuse disposal (see discussion of 8-cluster solution level below).

When all the lithics are combined into one class, cluster 3-3 has the highest percentage, with a total of $46 \%$ stone artefacts and $54 \%$ faunal materials. The location of cluster 3-3 near the front, or entrance of the shelter would also have been a more suitable location for tool manufacture as compared to the back of the shelter. Furthermore, Level II/2 accumulated at a time when the vertical height of the shelter ceiling may have been 
restricted near the back. Tool manufacture or repair would probably require more headroom as well as adequate lighting. Therefore the back of the site would probably be more suited to the deposition of food remains or associated debris, while activities such as tool making, food preparation and sleeping would have taken place closer to the drip line, towards the mouth of the shelter.

An investigation of the vertical distribution of the clusters is required for the evaluation of the three-dimensional relationships between them. The three-dimensional distribution of the level II/2 sampled dataset exhibits a wide vertical range (Appendix F Figure 1). A small group of artefacts in the deepest part of the level is visibly distinct from the majority of materials in the dataset; however, at this level of analysis (threecluster solution) this group of artefacts is not statistically different from cluster 3-3.

Three-dimensional plots were produced for each of the three clusters in the 3cluster solution (Appendix F - Figures 2-4) using the Trend Analysis Geostatistical Function in ArcGIS. When the clusters are examined three-dimensionally, there is very little vertical overlap indicating that the level is likely a single occupation phase. The clusters also tend to follow the natural slope of the site, which dips gradually from west to east and more sharply from the north (back) to south (mouth).

\subsubsection{3 - Level II/2: 8-Cluster Solution}

The 8-cluster solution for the level II/2 dataset effectively divides the contents of each cluster in the 3-cluster solution into two or three smaller artefact concentrations. In the 8-cluster solution, there is considerable overlap in the two-dimensional plan view between clusters 8-1 and 8-8, as well as some overlap of clusters towards the centre of 
the level. The spatial distribution of clusters demonstrates that most of the clusters are located in the centre and front of the shelter, with one cluster at the back (Appendix EFigure 2).

The most interesting feature about the 8-cluster solution is the overlap between cluster 8-1 and 8-8 in the western section of the site. The observed overlap may be the result of a natural vertical separation (site topography), or the presence of a palimpsest. The small group of finds, visually identified as spatially distinct but not statistically distinct in the 3 cluster solution (above), are identified as a separate cluster in the 8 cluster solution. This could indicate that the 3-cluster solution does not provide a high enough resolution for interpretation.

Cluster 8-1 is located in the western section of the site and contains 35 artefacts. The frequency and percentage of artefact types within cluster 8-1 are displayed below in table 7.5.

\begin{tabular}{|c|c|c|}
\hline Urtefact Iype & Treaviency Coun & Dercentage of Total \\
\hline Bone & 16 & 45.7 \\
\hline Chips and Flakes & 16 & 45.7 \\
\hline Stone Tools & 3 & 8.6 \\
\hline Total & 35 & 100.0 \\
\hline
\end{tabular}

Table 7.5: Cluster 8-1 Artefact Frequency and Percentage Distribution for the $Z$ Expansion Factor-10 8 Cluster Solution, Level II/2

Cluster 8-1 overlaps with the distribution of cluster 8-8 along the western perimeter of the excavated level, as already noted, which reinforces the impression that this is either a palimpsest, a small cluster of finds that actually belong in level III, or the result of carnivore activities mixing the deposits (see below).

Cluster 8-2 is a relatively small concentration of materials lying in the northwest section of the shelter, located horizontally between clusters 8-1 and 8-7. A total of 31 
artefacts are identified as cluster 8-2, which contains a variety of faunal and lithic materials.

\begin{tabular}{|c|c|c|}
\hline Wratact Wroe & 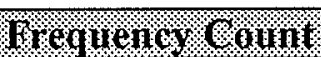 & 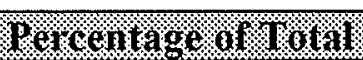 \\
\hline Bone & 23 & 74.2 \\
\hline Chips and Flakes & 6 & 19.4 \\
\hline Stone Tools & 2 & 6.5 \\
\hline Total & 31 & 100.0 \\
\hline
\end{tabular}

Table 7.6: Cluster 8-2 Artefact Frequency and Percentage Distribution for the $\mathrm{Z}$ Expansion Factor-10 8 Cluster Solution, Level II/2

Table 7.6 above displays the contents of cluster $8-2$. The high percentage of faunal materials in this cluster indicates that this may be representative of a butchering, processing or refuse area for faunal materials or a location to discard of materials from those activities, since it is located centrally but towards the back of the shelter (see discussion, below).

Cluster 8-3 is a smaller cluster containing 17 artefacts, located in the centre of the site towards the south. This cluster would probably be identified through visual analysis, as it is distinctly separated from the surrounding clusters. The frequency and percentage of artefact types in cluster 8-3 is presented below (Table 7.7).

\begin{tabular}{|c|c|c|}
\hline Artefact I Ype & Srequency Count & Hercentaro of 1 ordI \\
\hline Bone & 6 & 35.3 \\
\hline Chips and Flakes & 5 & 29.4 \\
\hline Stone Tools & 6 & 35.3 \\
\hline Total & 17 & 100.0 \\
\hline
\end{tabular}

Table 7.7: Cluster 8-3 Artefact Frequency and Percentage Distribution for the $Z$ Expansion Factor-10 8 Cluster Solution, Level II/2

Cluster 8-4 contains a larger concentration of artefacts located centrally, next to cluster 8-2 towards the back of the shelter. This cluster displays a slight overlap of $4 \mathrm{~cm}$ with cluster 8-7 in the horizontal distribution, which may be the result of a natural dip in 
the bedrock. The contents of cluster 8-4 are displayed below (Table 7.8). Faunal materials are the dominant artefact type in cluster 8-4.

\begin{tabular}{|l|c|c|}
\hline Direfict Type & Trequency Count & Percentage of Total \\
\hline Bone & 22 & 62.9 \\
\hline Chips and Flakes & 5 & 14.3 \\
\hline Stone Tools & 8 & 22.9 \\
\hline Total & $\mathbf{3 5}$ & $\mathbf{1 0 0 . 0}$ \\
\hline
\end{tabular}

Table 7.8: Cluster 8-4 Artefact Frequency and Percentage Distribution for the $Z$ Expansion Factor-10 8 Cluster Solution, Level II/2

Cluster 8-5 is a small cluster located along the eastern perimeter of the excavated area. A total of 16 artefacts are identified as belonging to cluster 8-5, which could be visually identified as a distinct cluster, though hard to differentiate from cluster 8-4 due to their spatial proximity. Table 7.9 displays the contents of cluster 8-5. Faunal materials represent half of the entire cluster contents.

\begin{tabular}{|l|c|c|}
\hline Artefact Mype & Trenuency Count & Rercentage of Total \\
\hline Bone & 8 & 50.0 \\
\hline Chips and Flakes & 4 & 25.0 \\
\hline Stone Tools & 4 & 25.0 \\
\hline Total & $\mathbf{1 6}$ & $\mathbf{1 0 0 . 0}$ \\
\hline
\end{tabular}

Table 7.9: Cluster 8-5 Artefact Frequency and Percentage Distribution for the $Z$ Expansion Factor-10 8 Cluster Solution, Level II/2

Cluster 8-6 is the smallest cluster in the solution and consists of a vertically distinct group of artefacts in the southeast section of the level. The 4 artefacts identified as cluster 8-6 would most likely not be identified as a separate, distinct cluster through visual inspection of the level II/2. Table 7.10 below displays the frequency and percentage distribution of artefact types within cluster 8-6. 


\begin{tabular}{|c|c|c|}
\hline Artefact Tyo & Hequency count & 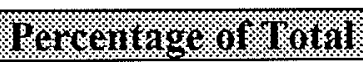 \\
\hline Bone & 3 & 75.0 \\
\hline Chips and Flakes & 1 & 25.0 \\
\hline Stone Tools & 0 & 0.0 \\
\hline Total & 4 & 100.0 \\
\hline
\end{tabular}

Table 7.10: Cluster 8-6 Artefact Frequency and Percentage Distribution for the $\mathrm{Z}$ Expansion Factor-10 8 Cluster Solution, Level II/2

The relatively small size of this cluster makes it difficult interpret based on either the cluster contents, or the horizontal distribution of artefacts.

A total of 27 artefacts are identified as cluster 8-7, located at the very back of the shelter. Table 7.11 (below) displays the frequency and percentage of artefact types:

\begin{tabular}{|c|c|c|}
\hline 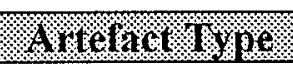 & Hernenuy / vount & Percentage ol rolat \\
\hline Bone & 19 & 70.4 \\
\hline Chips and Flakes & 7 & 25.9 \\
\hline Stone Tools & 1 & 3.7 \\
\hline Total & 27 & 100.0 \\
\hline
\end{tabular}

Table 7.11: Cluster 8-7 Artefact Frequency and Percentage Distribution for the $\mathrm{Z}$ Expansion Factor-10 8 Cluster Solution, Level II/2

Clusters 8-7, 8-4 and 8-2 comprise cluster 3-2 in the 3-cluster solution for level II/2. Clusters 8-2, 8-4 and 8-7 are collectively dominated by faunal materials, which suggest that this area (back of the shelter) may have been used for the disposal of bone refuse (see below).

Cluster $8-8$ is located along the western perimeter of level II/2 and demonstrates considerable overlap with the contents of cluster 8-1 (noted above). A total of 16 artefacts are identified as cluster 8-8, with faunal materials representing the majority of the cluster contents. Table 7.12 below displays the frequency and percentage distribution of artefact types: 


\begin{tabular}{|c|c|c|}
\hline Aitufact Yoo & Hequengy (ount & 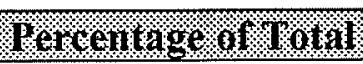 \\
\hline Bone & 10 & 62.5 \\
\hline Chips and Flakes & 4 & 25.0 \\
\hline Stone Tools & 2 & 12.5 \\
\hline Total & 16 & 100.0 \\
\hline
\end{tabular}

Table 7.12: Cluster 8-8 Artefact Frequency and Percentage Distribution for the $Z$ Expansion Factor-10 8 Cluster Solution, Level II/2

\subsubsection{4 - Significance}

Based on the small size of clusters in the 8-cluster solution, a chi-square significance test could not be performed for all cluster pairs, as they did not meet the requirements outlined in chapter 5 . Of the 28 cluster pairs that could be analyzed, cluster pairs 3 (comparing clusters 8-1 and 8-4), 8 (8-2 and 8-3) and 17 (8-3 and 8-7) were determined to be significantly different in contents. The remaining cluster pairs did not reject the null hypothesis, indicating that they contain statistically similar frequencies of artefact types. When clusters 8-1 and 8-4 are compared, it is clear that cluster 8-4 has a greater percentage of faunal materials and stone tools, while 8-1 has a higher frequency of stone flakes. Cluster 8-4 is located in the centre of the level; while cluster 8-1 occupies the western section towards the front indicating that knapping took place more frequently towards the front of the shelter

When clusters 8-2 and 8-3 are compared, there is a higher percentage of faunal materials in cluster 8-2, while cluster 8-3 has a greater percentage of stone tools. Cluster $8-2$ is located along the back wall, while cluster $8-3$ is located in the centre of the level where stone flakes and tools demonstrate the greatest concentration.

Clusters 8-3 and 8-7 were the final pair identified as significantly different in content. Cluster 8-3 contains a high percentage of stone tools and flakes, with a 
comparatively lower percentage of faunal materials. Cluster 8-7 demonstrates the opposite characteristics, with a greater percentage of faunal materials and very low percentages of stone tools and flakes. Cluster 8-7 is located at the back of the shelter in an area of higher artefact concentration, where faunal materials are generally present in higher frequencies. Conversely, cluster 8-3 is located in the centre of the site, where stone artefacts tend to dominate.

In summary, stone tools tend to be located in the centre of the level (cluster 8-3) and stone flakes are more associated with cluster 8-1 at the front of the shelter, which provides evidence that this would be a suitable area for the manufacture or repair of stone tools. Similarly, the concentration of faunal materials in clusters 8-2 and 8-7 at the back of the shelter suggests that this area may have been used to dispose of food remains. Faunal materials and stone tools in 8-4 could support the identification of either bone refuse disposal or a processing area in the central area of the shelter, as the lower ceiling would not have been conducive to any activities that required headspace or light. Sufficient evidence exists to state that the site was used differentially for the disposal of food waste and tool manufacture. The absence of burnt bone concentrations prohibits the identification of a fire pit or hearth within the level, making it difficult to determine the relationship of these activities to activities such as cooking or sleeping.

One of the most interesting features of the level $\mathrm{I} / 28$ - cluster solution is the potential identification of an archaeological palimpsest (Appendix F - Figures 5-12). There is a visible concentration of artefacts in the western section of the site. While the 3cluster solution grouped artefacts in this concentration into one large cluster, the 8-cluster solution separated this distribution into two smaller overlapping clusters (cluster 8-1 and 
8-8). The three-dimensional distributions of these clusters places cluster 8-8 (vertical range: $-128 \mathrm{~cm}$ to $-137 \mathrm{~cm}$ depth below datum) directly on top of cluster $8-1(-138 \mathrm{~cm}$ to $-153 \mathrm{~cm}$ depth below datum) separated by approximately $1 \mathrm{~cm}$ of sediment; this suggests that they share the same horizontal space, but are vertically distinct (Appendix F Figures 5 and 12). The contents of cluster 8-1 and 8-8 are not significantly different, supporting the possibility that they may represent two distinct phases of occupation, or the result of disturbance in the area (possibly due to carnivore activities).

Clusters 8-4, 8-5 and 8-7 are superimposed in the centre of the site, however, the overlap is most likely a product of the natural slope of the level as inspection of the threedimensional plots indicates (bedrock inclines are indicated on the plots) (Appendix FFigures 8,9 and 11). The clusters are not positioned directly above or below one another, which would indicate successive occupational/depositional episodes; instead, only a few artefacts actually demonstrate overlap.

In conclusion, differential use of space in level $\Pi / 2$ is demonstrated with food processing debris being tossed towards the back, as well as a central area (cluster 8-4) of approximately $4 \mathrm{~m}^{2}$ where bone processing may have taken place. Lithic manufacture and rejuvenation would have taken place centrally towards the front of the shelter (cluster 8-1).

\subsection{2 - Level III}

The results of the $\mathrm{K}$-means cluster analysis combined with the $\mathrm{Z}$ expansion factor10 identified optimal cluster solutions at 2, 4 and 6 clusters. As mentioned within Chapter 6 , the results of the 2-cluster solution were determined to be a product of the nature of the site formation processes (i.e. slope) and not an accurate representation of the potential 
spatial organization of activities. Therefore, the results of the 2-cluster solution will not be considered as part of the discussion.

Faunal materials represent approximately half of the entire dataset, with distributions concentrated along the outer edges of the level (Appendix B - Figures 5-8). There are visible concentrations of materials along the eastern and northeast perimeters however there are very few bones within the centre of the level. The spatial pattern of stone tools is quite different, with the greatest number of tools concentrated within the centre of the level. Stone Flakes are generally dispersed throughout the shelter; however, they are mainly concentrated towards the mouth of the shelter.

\subsubsection{1 - Level III: 4-Cluster Solution}

The arrangement of artefacts in the 4-cluster solution creates 4 concentrations of relatively similar size, which contain various frequencies of faunal materials, Stone Tools, Stone Flakes and Chips. The distribution of clusters divides the level into four sections, with clusters located in the west, north, east and southeast (Appendix E - Figure

3). The spatial distribution of each cluster is generally distinct, however there is considerable overlap between clusters towards the centre of the level. An interesting feature of the level III dataset is the visible concentration of materials along the eastern perimeter of the site. There is also a less obvious concentration, which follows the northwest perimeter, with a noticeable decline in artefact frequencies across the centre of the level, from the northeast to southwest. 
Cluster 4-1 is concentrated along the eastern perimeter with a total of 66 artefacts Two-dimensional visual identification of this cluster is difficult. The frequency and distribution of artefact types within cluster 4-1 are displayed below as table 7.13:

\begin{tabular}{|l|c|c|}
\hline \multicolumn{1}{|c|}{ Artefrat Tyes } & Frequency Cono & Percentage of Tolal \\
\hline Bone & 34 & 51.5 \\
\hline Chips and Flakes & 18 & 27.3 \\
\hline Stone Tools & 14 & 21.2 \\
\hline Total & $\mathbf{6 6}$ & $\mathbf{1 0 0 . 0}$ \\
\hline
\end{tabular}

Table 7.13: Cluster 4-1 Artefact Frequency and Percentage Distribution for the $Z$ Expansion Factor-10 4 Cluster Solution, Level III

Cluster 4-2 contains 59 artefacts in the northern section of the level, which would be difficult to identify visually. There are comparatively fewer faunal materials within cluster 4-2 as compared to the other clusters in the solution and a significantly higher frequency of stone tools (see Table 7.14 below).

\begin{tabular}{|l|c|c|}
\hline \multicolumn{1}{|c|}{ Arteract Type } & Trequency Count & Percentage of Total \\
\hline Bone & 19 & 32.2 \\
\hline Chips and Flakes & 24 & 40.7 \\
\hline Stone Tools & 16 & 27.1 \\
\hline Total & $\mathbf{5 9}$ & $\mathbf{1 0 0 . 0}$ \\
\hline
\end{tabular}

Table 7.14: Cluster 4-2 Artefact Frequency and Percentage Distribution for the $Z$ Expansion Factor-10 4 Cluster Solution, Level III

Cluster 4-3 is located in the western section of the site, with a total of 60 artefacts.

This cluster is likely identifiable through two-dimensional visual inspection with the exception of a few dispersed artefacts in the centre. The frequency of artefacts within cluster 4-3 is quite different from cluster 4-2 (Table 7.15 below).

\begin{tabular}{|l|c|c|}
\hline Artefac Type & Rrequency Cound & Fercentage of Towal \\
\hline Bone & 34 & 56.7 \\
\hline Chips and Flakes & 13 & 21.7 \\
\hline Stone Tools & 13 & 21.7 \\
\hline Total & $\mathbf{6 0}$ & $\mathbf{1 0 0 . 0}$ \\
\hline
\end{tabular}

Table 7.15: Cluster 4-3 Artefact Frequency and Percentage Distribution for the Z Expansion Factor-10 4 Cluster Solution, Level III 
Cluster 4-4 contains 63 artefacts concentrated along the southeast perimeter of the excavated level, which demonstrates some overlap with cluster 4-1. Table 7.16 below displays the contents of cluster 4-4. There is a noticeable decrease in the number of stone tools within this cluster.

\begin{tabular}{|c|c|c|}
\hline Nrefact I por & 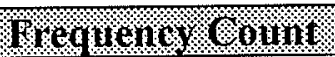 & 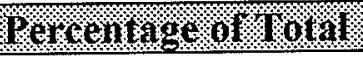 \\
\hline Bone & 26 & 41.3 \\
\hline Chips and Flakes & 33 & 52.4 \\
\hline Stone Tools & 4 & 6.3 \\
\hline Total & 63 & 100.0 \\
\hline
\end{tabular}

Table 7.16: Cluster 4-4 Artefact Frequency and Percentage Distribution for the $Z$ Expansion Factor-10 4 Cluster Solution, Level III

\subsubsection{2 - Significance}

The Chi-square significance tests for the 4-cluster solution indicated that cluster pairs $3,4,5$ and 6 rejected the null hypothesis. Cluster 4-1 is not significantly different from clusters 4-2 and 4-3, however it is significantly different from cluster 4-4. The frequency and percentage of artefacts in clusters 4-2, 4-3 and 4-4 are significantly different and therefore do not represent random artefact distributions. Cluster 4-2 has the highest frequency of stone tools located at the back of the shelter, while cluster 4-4 has the highest frequency of stone flakes and the lowest number of stone tools in the entrance. Clusters 4-1 and 4-3 contain higher percentages of faunal materials, however, there are also relatively higher numbers of stone tools compared to cluster 4-4.

A small concentration of burnt bone was identified in the centre of the site towards the west (Appendix A - Figure 9). While this does not provide direct evidence for the location of a hearth, it does provide evidence for the use of fire within the level. The location of the burnt bone concentration in the centre of the site could suggest that 
the vertical height of the shelter may have been greater than in level II/2, enabling people to make full use of the shelter. The high frequency of stone tools and flakes located near the back of the shelter would support this observation. Stone tools are concentrated in the area surrounding the location of the burnt bone (cluster 4-3). If the original hearth was located in this area, the presence of stone tools in association with burnt bone supports the suggestion that stone tool repair and manufacture took place in this area, requiring the availability of light and sufficient headroom. Cluster $4-4$, in the front of the shelter, contains very few stone tools, indicating that tool use and manufacture may have been located towards the back of the shelter. This is also supported by the high frequency of stone tools and flakes and the small percentage of bone within cluster 4-2. There are very few faunal materials in the centre of the site. These materials may have been deposited or cleared towards the walls of the shelter (especially on the eastern side) where they would be out of the way. Cluster 4-4 also contains the highest concentration of stone flakes, which may have been discarded as refuse and then deposited or moved towards the edges of the shelter with the bone refuse.

The three-dimensional distribution of materials in level III demonstrates a generally concentrated pattern, which follows the natural slope from north to south and west to east (Appendix F - Figure 13) suggesting they are contemporary, or deposited within the same occupation period. Visual examination of the dataset also identifies a small group of artefacts in the deepest section of the level, which appear to be spatially distinct from the main concentration of materials. While they are visibly distinct at this level of resolution, they are not statistically distinct. The three-dimensional distribution of clusters (Appendix F - Figures 14-17) provides minimal evidence of a potential vertical 
separation of $2 \mathrm{~cm}$ between clusters $4-1$ and $4-4$ in the centre of the site. It is difficult to determine whether the overlap is the result of the natural slope of the site or whether it represents an actual palimpsest level (Appendix F - Figures 14 and 17). The vertical distribution of cluster 4-4 also demonstrates some interesting characteristics (Appendix F - Figure 17), as there appears to be a distinct vertical separation between artefacts in the cluster, which are evaluated below through the examination of the 6-cluster solution.

\subsubsection{3 - Level III: 6-Cluster Solution}

The 6-cluster solution creates a series of small clusters of comparable size (Appendix E - Figure 4). The two-dimensional horizontal distribution of individual clusters subdivides the site into 6 sections, with two clusters in the southeast, one larger cluster in the north and three clusters, which run across the centre. When contrasted with the results of the 4-cluster solution, it was determined that clusters 4-1 and 4-3 are divided into three clusters, while cluster 4-4 is separated into 2 individual concentrations. The 6-cluster solution demonstrate considerable overlap between clusters, especially towards the centre.

Cluster 6-1 is located along the eastern perimeter of the level and contains 45 artefacts. The cluster would probably be identifiable through visual inspection. Table 7.17 (below) displays the distribution of artefact types within cluster 6-1. Faunal materials represent approximately half of the total cluster contents. 


\begin{tabular}{|c|c|c|}
\hline 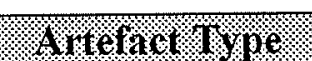 & Tregrarney count & Percentage of trotal \\
\hline Bone & 24 & 53.3 \\
\hline Chips and Flakes & 12 & 26.7 \\
\hline Stone Tools & 9 & 20.0 \\
\hline Total & 45 & 100.0 \\
\hline
\end{tabular}

Table 7.17: Cluster 6-1 Artefact Frequency and Percentage Distribution for the $Z$ Expansion Factor-10 6 Cluster Solution, Level III

Cluster 6-2 contains 54 artefacts located centrally, in the northern section (back of the shelter). Visual inspection of level III did not identify the presence of cluster 6-2.

There is also some potential overlap with clusters 6-1 and 6-3 in the centre of the level. There is a comparatively lower percentage of faunal materials in this cluster (Table 7.18 below).

\begin{tabular}{|l|c|c|}
\hline Arrefact Type & Trequency Crinu & Percentige of Tolal \\
\hline Bone & 17 & 31.5 \\
\hline Chips and Flakes & 22 & 40.7 \\
\hline Stone Tools & 15 & 27.8 \\
\hline Total & $\mathbf{5 4}$ & $\mathbf{1 0 0 . 0}$ \\
\hline
\end{tabular}

Table 7.18: Cluster 6-2 Artefact Frequency and Percentage Distribution for the $Z$ Expansion Factor-10 6 Cluster Solution, Level III

Cluster 6-3 is located in the centre of the level, with 41 artefacts within the western and eastern sections of the cluster distribution. This cluster was not visibly distinguishable as a distinct cluster. There is also a considerable degree of overlap with cluster 6-2 and 6-5. The distribution of artefact types is displayed below in Table 7.19:

\begin{tabular}{|l|c|c|}
\hline Artefact Trpe & Irequercy Count & Percentage of Total \\
\hline Bone & 11 & 26.8 \\
\hline Chips and Flakes & 16 & 39.0 \\
\hline Stone Tools & 14 & 34.1 \\
\hline Total & $\mathbf{4 1}$ & $\mathbf{1 0 0 . 0}$ \\
\hline
\end{tabular}

Table 7.19: Cluster 6-3 Artefact Frequency and Percentage Distribution for the $Z$ Expansion Factor-10 6 Cluster Solution, Level III 
Cluster 6-4 contains a total of 48 artefacts in the southeast corner of the level.

This cluster is easily identified by visual inspection; however, it would probably be combined with artefacts from cluster $6-6$, as there is a slight overlap of artefacts. This separation suggests that the $\mathrm{k}$-means program identified an important vertical distinction. Within cluster 6-4, faunal materials represent the majority of the artefacts (see table 7.20 below) and there are comparatively few stone flakes and only one stone tool.

\begin{tabular}{|c|c|c|}
\hline Artefact I Ype & Trequenu voung & 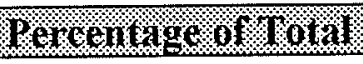 \\
\hline Bone & 33 & 68.8 \\
\hline Chips and Flakes & 14 & 29.2 \\
\hline Stone Tools & 1 & 2.1 \\
\hline Total & 48 & 100.0 \\
\hline
\end{tabular}

Table 7.20: Cluster 6-4 Artefact Frequency and Percentage Distribution for the $Z$ Expansion Factor-10 6 Cluster Solution, Level III

Cluster 6-5 contains 40 artefacts in the western section of the level and is visibly identifiable. The contents of cluster 6-5 are displayed in Table 7.21 (below):

\begin{tabular}{|c|c|c|}
\hline Mrtefact IYpe & Hogurargroun & Sercentage ol Yolal \\
\hline Bone & 25 & 62.5 \\
\hline Chips and Flakes & 9 & 22.5 \\
\hline Stone Tools & 6 & 15.0 \\
\hline Total & 40 & 100.0 \\
\hline
\end{tabular}

Table 7.21: Cluster 6-5 Artefact Frequency and Percentage Distribution for the $Z$ Expansion Factor-10 6 Cluster Solution, Level III

Cluster 6-6 is the smallest cluster in the solution, located along the southern perimeter of the level, with a total of 20 artefacts. Visual identification combines the distribution of cluster 6-6 with cluster 6-4 to form one larger cluster. Cluster 6-6 demonstrates a unique distribution of artefacts. While the cluster is comparatively small, there are very few faunal materials within the cluster and a significantly higher percentage of stone flakes (see table 7.22 below). 


\begin{tabular}{|c|c|c|}
\hline Wrtatathor & Hrequenur cruit & 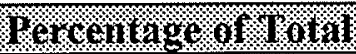 \\
\hline Bone & 3 & 15.0 \\
\hline Chips and Flakes & 15 & 75.0 \\
\hline Stone Tools & 2 & 10.0 \\
\hline Total & 20 & 100.0 \\
\hline
\end{tabular}

Table 7.22: Cluster 6-6 Artefact Frequency and Percentage Distribution for the $Z$ Expansion Factor-10 6 Cluster Solution, Level III

\subsubsection{4 - Significance}

The results of the Chi-square significance tests for the 6-cluster solution indicate that the contents of cluster 6-1 are significantly different from clusters $6-3,6-4$ and $6-6$; cluster 6-2 is significantly different from clusters $6-4,6-5$ and 6-6 and the contents of cluster 6-3 are statistically different from clusters $6-4,6-5$ and 6-6. Cluster 6-6 is significantly different from all other clusters in the solution.

A comparison of the contents of individual clusters in the solution indicates that cluster 6-2 has the highest frequency of stone tools. There is also a high frequency of stone flakes and stone tools within cluster $6-3$. Cluster $6-4$ has the highest frequency of bone, however, it also has the lowest number of stone tools. As indicated by the results of the chi-square test, the contents of clusters 6-1 and 6-5 are generally comparable; both contain higher percentages of faunal materials. Cluster 6-6 contains the lowest percentage of bone and the highest percentage of stone flakes.

Those clusters with the highest frequency of stone tools and lower bone counts (clusters 6-2 and 6-3) are located within the centre of the level, while clusters with higher percentages of bone (clusters 6-1, 6-4 and 6-5) are positioned towards the outer edges of the excavated level. Cluster 6-6 presents a completely different configuration, with stone 
flakes representing the highest frequency of artefacts in the cluster; it is located at the front of the shelter.

Clusters 6-2, 6-3 and 6-5 are located around the burnt bone concentration mentioned above (Appendix A - Figure 9). Their spatial separation suggests that there were several activity areas at the centre of the occupation. The higher frequency of stone tools and flakes in cluster 6-2, near the back of the shelter, suggests that tools may have been manufactured or repaired in this location. The presence of stone tools associated with burnt bone suggests that individuals positioned themselves around the fire while working in order to obtain heat and light.

The absence of faunal materials in the centre of the site and the higher frequency towards the edges suggests that refuse may have been deposited or pushed towards the walls of the shelter. It is also possible that animals were processed in these areas away from the centre of the level, where activities such as cooking and sleeping could have occurred. This is further supported by the fact that bone and stone flakes are present in greater frequencies in clusters 6-4 and 6-6. As described previously, stone flakes and faunal materials demonstrate the same pattern of spatial distribution, with concentrations occurring on the edges of the site, supporting the suggestion that the outer edges of the shelter were used for refuse disposal.

The three-dimensional spatial distribution of the 6-cluster solution revealed the potential presence of a palimpsest (Appendix F - Figures 13, 18-23). The distribution of artefacts is generally concentrated, however, there is a small group (cluster 6-6) of vertically distinct artefacts (Appendix F - Figure 13). Cluster 6-4 demonstrates horizontal overlap with cluster $6-6$, with a vertical separation of approximately $2 \mathrm{~cm}$ between them. 
Cluster 6-4 lies between $-166 \mathrm{~cm}$ to $-178 \mathrm{~cm}$ (depth below datum) and 6-6 lie between $180 \mathrm{~cm}-189 \mathrm{~cm}$ (depth below datum) (Figure 7.1 below):

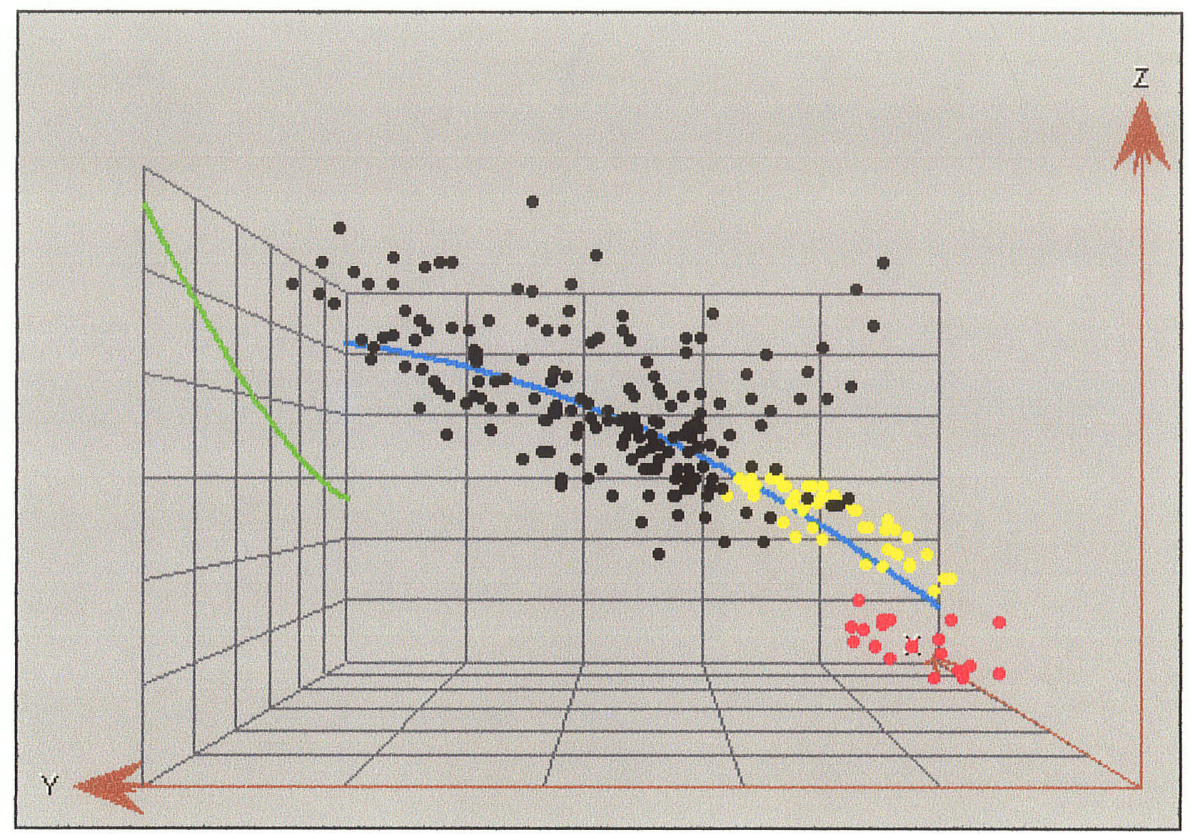

Figure 7.1: Three-Dimensional Distribution Level III - Vertical Overlap Between Clusters 6-4 (highlighted in yellow) and 6-6 (highlighted in red)

When the three-dimensional distribution of cluster 6-6 is compared with the remaining clusters in the solution, it demonstrates spatial segregation, which indicates that this may be a palimpsest of materials. Cluster 6-6 is comparatively small, with a total of only 21 artefacts, suggesting it may have been part of a larger deposit, rather than an individual occupation. It is also possible that cluster 6-6 may represent a small group of miss-assigned artefacts belonging to level 4 . The vertical distribution of these artefacts supports this interpretation. Cluster 6-6 has a vertical range of $180 \mathrm{~cm}$ to $189 \mathrm{~cm}$ depth below datum, while level 4, in squares 10J and 11J (the location of cluster 6-6) has a vertical distribution of $185 \mathrm{~cm}$ to $196 \mathrm{~cm}$ depth below datum. 
The remaining clusters in the 6-cluster solution follow the natural slope of the level III distributions, with a little overlap occurring in the centre of the site. The twodimensional (horizontal) distribution makes it appear that 6-3 and 6-5 overlap considerably. Upon examination in three dimensions, this overlap is still visible, but it can be explained as a product of the natural slope of the site.

In conclusion, cluster analysis has revealed differential use of space in level IIII. Bone refuse and debris are distributed along the outer edges of the site (clusters 6-1, 6-4 and 6-5), while bone processing (cluster 6-2 and 6-3) may have taken place in conjunction with tool making (cluster 6-2) around the hearth, located centrally towards the mouth of the shelter.

\subsection{3 - Level IV}

Optimal cluster solutions are identified at 3 and 5 clusters for level IV. Level IV presents a challenge with respect to the analysis of the spatial patterning of artefacts. The natural process of brecciation, i.e. carbonate cementation of materials has occurred at varying degrees across level IV (Yevtushenko et al. In Press a and b). In addition, the two-dimensional horizontal distribution of the level IV dataset suggests that archaeological materials in the southeast and western sections may not have been completely excavated (Appendix B - Figures 9-12). There is a significant concentration of artefacts along the eastern and southern baulks indicating that the distribution continues past the excavated area. This is a boundary problem for spatial analysis, which relates to the chapter 2 discussion. It is necessary to consider this sampling problem when interpreting the contents of clusters in these areas. 
The horizontal distribution of archaeological materials demonstrates unique spatial patterning with respect to artefact type. Faunal materials represent approximately half of the entire dataset. Materials are generally dispersed throughout the level with greater concentrations towards the outer edges of the east and west sections (Appendix B-Figure 12). There are comparatively few fauna within the centre of the site, with a complete absence of materials within the south-central section. The concentration and distribution of stone flakes demonstrates a more random distribution throughout the level, with the majority of artefacts dispersed throughout the southern half of the site (Appendix B - Figure 11). The spatial relationship between faunal materials and stone flakes is similar to level III (discussed above).

The horizontal distribution of stone tools appears to be random when compared to the distributions of faunal materials and stone flakes (Appendix B - Figure 10). There is a visible concentration of tools in the southwestern section of the site as well as a group of 6 tools, which are spatially distinct in the northern section of the level.

\subsubsection{1 - Level IV: 3-Cluster Solution}

The 3-cluster solution creates a group large clusters, which are relatively equal in size and spatial distribution, containing a variety of faunal materials, stone chips, stone flakes and stone tools (Appendix E - Figure 5). The position of clusters within the level subdivides the site into northern, southwest and southeast sections. Clusters 3-1 and 3-3 are visibly dispersed while cluster 3-2 appears more concentrated.

Cluster 3-1 contains a total of 84 artefacts and is located in the southeast part of the level. This cluster occupies a large space of approximately $9 \mathrm{~m}^{2}$ and is relatively 
diffuse. Cluster 3-1 would probably be visually identified as a series of small, spatially distinct clusters, rather than one large concentration. Cluster 3-1 also demonstrates horizontal overlap with cluster 3-3 in the centre of the level, towards the back. Table 7.23 (below) displays the frequency and percentage distribution of artefact types within cluster 3-1:

\begin{tabular}{|l|c|c|}
\hline Artefact Type & Irequenc Coun & Percentiage of rotal \\
\hline Bone & 36 & 42.9 \\
\hline Chips and Flakes & 39 & 46.4 \\
\hline Stone Tools & 9 & 10.7 \\
\hline Total & $\mathbf{8 4}$ & $\mathbf{1 0 0 . 0}$ \\
\hline
\end{tabular}

Table 7.23: Cluster 3-1 Artefact Frequency and Percentage Distribution for the $Z$ Expansion Factor-10 3 Cluster Solution, Level IV

Cluster 3-2 contains a total of 92 artefacts located in the western section of the level. This cluster is the largest cluster in the solution and consequently the most spatially concentrated. Cluster 3-2 is easily distinguishable through two-dimensional visual inspection as the majority of artefacts within the cluster are spatially distinct and concentrated. Table 7.24 (below) displays the contents of cluster 3-2:

\begin{tabular}{|c|c|c|}
\hline Arofact Woc & Mrequency Gount. & Oercontage af rotal \\
\hline Bone & 42 & 45.7 \\
\hline Chips and Flakes & 37 & 40.2 \\
\hline Stone Tools & 13 & 14.1 \\
\hline Total & 92 & 100.0 \\
\hline
\end{tabular}

Table 7.24: Cluster 3-2 Artefact Frequency and Percentage Distribution for the $Z$ Expansion Factor-10 3 Cluster Solution, Level IV

Cluster 3-3 is the smallest cluster in the solution, with a total of 63 artefacts located in the northern part of the level. The cluster would most likely be identified through visual inspection, as it is spatially distinct from the other clusters in the solution. The frequency and percentage of artefacts within cluster 3-3 are unique when compared with the other clusters in the solution (Table 7.25 below). 


\begin{tabular}{|c|c|c|}
\hline Wrtefact 1 Yre & Grequero Court. & Percentage ol Woul \\
\hline Bone & 35 & 55.6 \\
\hline Stone Flakes & 20 & 31.7 \\
\hline Stone Tools & 8 & 12.7 \\
\hline Total & 63 & 100.0 \\
\hline
\end{tabular}

Table 7.25: Cluster 3-3 Artefact Frequency and Percentage Distribution for the $Z$ Expansion Factor-10 3 Cluster Solution, Level IV

\subsubsection{2 - Significance}

The Chi-square significance tests determined that none of the clusters in the 3cluster solution level are significantly different in content. This means that the contents of each cluster in the solution are comparable and represent a random distribution of artefacts. It could indicate that activities within the shelter are not spatially distinct (see Binford 1996). If small groups or individuals continually occupied the shelter over a period of time, the spatial distribution of artefacts would display concentrations of small assemblages containing the same variety of artefacts (Simek et al. 1985: 231). Clusters 32 and 3-3 occupy relatively large area of approximately $6 \mathrm{~m}^{2}$, which means that they could represent a series of ephemeral occupations.

While the statistical results determined that there was no significant difference between the contents of individual clusters in level IV at the three-cluster solution level, it is possible to make some general observations about the distribution of materials across the level. Faunal materials and stone flakes are generally concentrated towards the outer perimeters, with the exception of cluster $3-3$, where stone tools are located along the northern perimeter. The majority of stone tools appear to be concentrated in the centre of the site towards the western section of the level. There is a marked separation between the distribution of stone tools across the site and the small concentration of tools in 
cluster 3-3. This could support the interpretation of the three clusters as individual occupations (containing similar artefacts).

Burnt bone was recovered from level IV, with one large concentration in the centre of the shelter towards the west (Appendix A-Figure 10). This does not mean a hearth was located there, however it does indicate that fire was being used at the shelter during the time of deposition of level IV. The large concentration of burned bone in the centre of the site suggests that this location would be the most probable hearth location. Smaller concentrations surrounding this area suggest that the shelter was perhaps used more than once. As mentioned above, stone tools are concentrated centrally, towards the west and a fire would have provided light and warmth.

When the artefact distribution for level IV is compared with the stratigraphic profile of the west wall, it is clear that the lithological strata containing level IV slope from north to south (Chapter Four - Figure 4.2). The distribution of the level IV dataset (e.g. small overlap of clusters 3-1 and 3-3) follows the natural slope of the shelter floor at the time of deposition, prior to the accumulation of successive layers of eboulis (Appendix F - Figure 24).

The three dimensional distribution of the 3-cluster solution displays relatively concentrated, spatially distinct artefact clusters (Appendix F - Figures 25-27). Within the horizontal profile (Appendix E - Figure 5) overlap was identified between clusters 3-1 and 3-3, however a three-dimensional comparison of these clusters suggests that the overlap is the result of natural slope, as the clusters do not demonstrate successive deposition (one cluster above the other). 


\subsubsection{3 - Level IV: 5-Cluster Solution}

In the 5-cluster solution, the 3-cluster solution is further divided into smaller artefact concentrations, which are more spatially distinct (Appendix E - Figure 6). The contents of cluster 3-1 and 3-3 are divided into two smaller clusters, while the original distribution of cluster 3-2 stays the same. There is also visible overlap between some of the clusters in the solution, suggesting that there are unique vertical relationships that are worth exploring in a three dimensional analysis.

Cluster 5-1 is located along the eastern edge of level IV, with a total of 42 artefacts. There is considerable overlap between cluster 5-1 and 5-2 in the southeast corner. Cluster 5-1 would likely be visually identifiable as a distinct cluster. The contents of cluster 5-1 are displayed below (Table 7.26):

\begin{tabular}{|c|c|c|}
\hline Autefact Type & Mrequency Count & 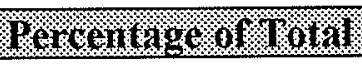 \\
\hline Bone & 19 & 45.2 \\
\hline Chips and Flakes & 22 & 52.4 \\
\hline Stone Tools & 1 & 2.4 \\
\hline Total & 42 & 100.0 \\
\hline
\end{tabular}

Table 7.26: Cluster 5-1 Artefact Frequency and Percentage Distribution for the $Z$ Expansion Factor-10 5 Cluster Solution, Level IV

Cluster 5-2 is the smallest cluster in the solution, consisting of 32 artefacts, located along the southern perimeter. This cluster is spatially distinct and would likely be visually identifiable. Table 7.27 (below) displays the contents of cluster 5-2, including the frequency and percentage of each artefact type.

\begin{tabular}{|c|c|c|}
\hline Nitefact YYoe & Frequend / Gourt & Perronlage ol / old \\
\hline Bone & 14 & 43.8 \\
\hline Chips and Flakes & 13 & 40.6 \\
\hline Stone Tools & 5 & 15.6 \\
\hline Total & 32 & 100.0 \\
\hline
\end{tabular}

Table 7.27: Cluster 5-2 Artefact Frequency and Percentage Distribution for the $\mathrm{Z}$ Expansion Factor-10 5 Cluster Solution, Level IV 
Cluster 5-3 contains 58 artefacts located in the centre of the level. This cluster occupies a large space and demonstrates considerable overlap with cluster 5-4. Visual identification of the cluster in two dimensions would be difficult. As indicated by Table 7.28 (below), cluster 5-3 contains a high frequency of stone flakes.

\begin{tabular}{|l|c|c|}
\hline Artefact Type & Trequency Count & Perentiage of Towal \\
\hline Bone & 23 & 39.7 \\
\hline Chips and Flakes & 26 & 44.8 \\
\hline Stone Tools & 9 & 15.5 \\
\hline Total & $\mathbf{5 8}$ & $\mathbf{1 0 0 . 0}$ \\
\hline
\end{tabular}

Table 7.28: Cluster 5-3 Artefact Frequency and Percentage Distribution for the $Z$ Expansion Factor-10 5 Cluster Solution, Level IV

A total of 35 artefacts were identified as cluster 5-4, located in the northern section of level IV. As mentioned above, cluster 5-3 and 5-4 overlap each other. These clusters would most likely be visibly identified as one large cluster. Table 7.29 below includes the frequency counts and percentage of each artefact type within cluster 5-4.

\begin{tabular}{|l|c|c|}
\hline Artefac Tye & Trequency Coun & Percentage of Toral \\
\hline Bone & 19 & 54.3 \\
\hline Stone Flakes & 10 & 28.6 \\
\hline Stone Tools & 6 & 17.1 \\
\hline Total & 35 & $\mathbf{1 0 0 . 0}$ \\
\hline
\end{tabular}

Table 7.29: Cluster 5-4 Artefact Frequency and Percentage Distribution for the $Z$ Expansion Factor-10 5 Cluster Solution, Level IV

Cluster 5-5 is the largest cluster in the solution, located exclusively within the western half of level IV. A total of 72 artefacts were identified as cluster 5-5, which demonstrates the greatest degree of clustering and would most likely be visually identified. Faunal materials represent just over half of the cluster, with approximately 53\% (Table 7.30 below): 


\begin{tabular}{|c|c|c|}
\hline A.tefict I Ype & 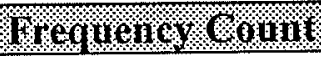 & 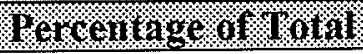 \\
\hline Bone & 38 & 52.8 \\
\hline Chips and Flakes & 25 & 34.7 \\
\hline Stone Tools & 9 & 12.5 \\
\hline Total & 72 & 100.0 \\
\hline
\end{tabular}

Table 7.30: Cluster 5-5 Artefact Frequency and Percentage Distribution for the $Z$ Expansion Factor-10 5 Cluster Solution, Level IV

\subsubsection{4 - Significance}

The results of the chi-square significance tests determined that clusters 5-1 and 54 are significantly different. Each of the remaining cluster pairs in the 5-cluster solution is statistically similar. A comparison of the contents of cluster 5-1 and 5-4 indicates that cluster 5-1 contains only 1 stone tool; however, it has a very high percentage of stone flakes. The percentage of stone flakes within cluster 5-4 is considerably lower, but also has a much higher percentage of faunal materials and stone tools. Cluster 5-1 is located along the eastern edge, suggesting that it may continue beyond the limits of the excavation. It is difficult to interpret the contents of this cluster in terms of occupational activities without knowing the true eastern boundary of the level, however, it clearly contains refuse from tool making and/or rejuvenation. Cluster 5-2 also appears to extend past the southern perimeter of the excavated area. The southern excavation boundary marks the probable entrance of the rock shelter, while the bench in front of the shelter has experienced considerable erosion. These clusters are clearly truncated, therefore, an interpretation of clusters 5-1 and 5-2 will not be developed further.

Faunal materials in level IV generally exhibit concentrations towards the outer edges of the level, specifically in the northwest. This is supported by the location of cluster $5-4$, which has the highest percentage of faunal materials. Stone flakes and stone 
tools demonstrate higher concentrations throughout the centre of the level, where there are notably fewer faunal materials. Stone tools also exhibit a small concentration in cluster 5-4 at the back of the shelter.

A large quantity of burnt bone was recovered from level IV and the greatest concentration is located in the centre of the shelter towards the west (Appendix AFigure 10). This is the most probable location for a hearth, however, smaller concentrations in the surrounding area may provide evidence of successive occupations at various locations within the shelter. The distribution of burned bone differs within levels 3 and 4 and therefore further suggests that the nature of these levels is different.

The three-dimensional distribution of the 5-cluster solution supports the possible interpretation of clusters 5-4 and 5-5 as the result of separate depositional events (Appendix F - Figures 28-32). There is vertical separation of approximately $13 \mathrm{~cm}$ between these clusters in the western section of the level. There is also a vertical separation of approximately $4 \mathrm{~cm}$ between clusters 5-1 and 5-2 in the southeast corner of level IV (Appendix F - Figures 28 and 29). This is indicative of successive occupations rather than contemporary deposition, however the total extent of the artefact distribution in this area is unknown.

Clusters 5-2 and 5-3 are positioned along the natural slope of the site, with cluster 5-3 located at a higher position than 5-2 (Appendix F - Figures 29 and 30). Therefore, the overlap is probably indicative of natural site formation processes. As discussed previously, the limitations associated with the spatial distribution of cluster 5-2 along the southern perimeter of level IV prohibit further examination of this relationship. The 
inclusion of additional data through continued excavation would likely modify current cluster distributions, as the position of the cluster centroids would change.

Cluster 5-3 and 5-4 also demonstrate a slight overlap. Cluster 5-4 contains a unique mix of stone tools and flakes for this level, which is associated with a concentration of burnt bone in the northern section of the site. When the threedimensional distribution of cluster 5-4 is examined, there is a clear vertical separation of approximately $3 \mathrm{~cm}$ between it and the remaining clusters (Appendix F - Figure 31). This supports the possibility that cluster 5-4 is representative of a separate depositional event, although it does follow the natural slope of the distribution. The significance test also determined that these clusters were statistically identical, further suggesting that they are representative of two unique depositional events.

Finally, cluster 5-5 is comparatively large, representing an area of approximately $6 \mathrm{~m}^{2}$ and demonstrates spatial segregation in all three dimensions, suggesting that it may represent either a distinct occupational episode or a spatially discrete area of the site, the function of which is not discernable as cluster contents are statistically identical to other clusters (Appendix F - Figure 32). In conclusion, the results of the k-means analysis for level IV produced distinct three-dimensional patterns in the dataset, which are best interpreted as a palimpsest deposit.

\section{4 - Conclusions}

On the basis of the foregoing discussion, a general pattern emerges whereby the back of the shelter and the peripheries of the excavated area seem to have been used consistently as refuse discard areas. Tool working seems to occur centrally and towards 
the shelter entrance, while bone processing and tool use areas are distinguishable in levels $\mathrm{II} / 2$ and 3 , with the area in level 3 occurring near a putative hearth.

As discussed in chapter 2, the investigation of spatial organization in Middle Palaeolithic occupations has resulted in an important debate comparing the behavioural organization of Neanderthals with that of modern humans (Binford 1996; Mellars 1996; Pettitt 1997; Vaquero and Pasto 2001; Vaquero 1999). While anatomically modern humans demonstrate unique spatial organization of activities around a central hearth, claims for the absence of such organization in Neanderthal sites has been interpreted as evidence for only a very basic degree of spatial organization (Binford 1996).

The results of the Kintigh's K-means cluster analysis discussed above provide sufficient evidence for the differential use of space within levels 2.2 and 3 at Karabi Tamchin. In both levels, there is a distinction between potential areas of refuse disposal (back and periphery of the shelter), bone processing and tool use/manufacture (centrally and at the shelter entrance), that suggests that individuals were performing activities in specific areas of the shelter. This indicates that Neanderthals were organizing their activities within the shelter, in direct contradiction to Binford's (1996) claims.

Researchers have also suggested that the use of space by non-human carnivores and Neanderthal populations is comparable (Pettitt 1997); however, evidence from Karabi Tamchin indicates that a distinction can be made. Within level II/2, the presence of carnivores has been confirmed by the archaeologists (Burke In Press; Yevtushenko et al. In Press a), indicating potential post-depositional disturbance of cultural materials. While it is possible that carnivores have disturbed the cultural patterns, areas of tool use and manufacture could still be identified in level II/2. Further support for a distinction 
between carnivore and hominid patterns at Karabi Tamchin is provided by the presence of burned bone concentrations in levels III and IV. As suggested by Vaquero and Pasto (2001: 1219), comparisons between non-human carnivores and Neanderthal populations do not consider the significance of hearths, which demonstrates complex cognitive capability comparable to modern human behaviour (based on ethnoarchaeological evidence), which cannot be associated with non-human carnivore behaviour. In level 3, the presence of burned bone is associated with a potential bone processing area, which can be distinguished from refuse areas. This evidence also refutes Binford's (1996) suggestion that Neanderthals do not demonstrate differential use of space within hearthrelated assemblages.

Burned bone concentrations were also identified in level IV, however the k-means cluster analysis suggests that level IV is a palimpsest within which special activity areas could not be identified. The process of carbonate cementation (brecciation) identified in level IV has also affected the distribution and recovery of archaeological materials, which means that the original patterning may no longer be distinguishable (Kroll and Price 1991: 197). The presence of a palimpsest in level IV at Karabi Tamchin does not indicate that individual occupations lacked complex spatial organization, but rather that horizontal spatial patterning has been obscured by a series of overlapping ephemeral occupations. The accumulation of debris from short-term occupations would have been minimal, making it difficult to identify specific activity areas due to sample size.

The organization of space within Middle Palaeolithic sites has also been interpreted as a pragmatic response to the environment, rather than evidence for cognitive or social complexity in Neanderthal populations (Mellars 1996). In other words, once 
again, Neanderthal assemblages would demonstrate patterning similar to that of nonhuman carnivores and natural site constraints would dictate the division of activity areas into areas used for living and refuse disposal (Mellars 1996: 311). Vaquero (1999) argues that all human behaviour is naturally pragmatic. It is possible, therefore, that spatial patterning does not constitute a sound basis for establishing cognitive complexity. Nevertheless, the results of this research demonstrate intra-level spatial patterning, which is clearly distinct from non-human carnivore behaviour. Activity areas are located centrally, in proximity to potential hearth locations, while refuse areas tend to be located towards the back of the shelter. This pattern is a logical (and indeed, pragmatic) arrangement of activities within a confined space, which would likely be employed by modern humans under similar constraints; therefore, it does not provide evidence against cognitive complexity in Middle Palaeolithic populations.

Evidence for the organization of space by prehistoric hominids presented above, suggests that further investigation and interpretation of spatial patterning within cave and rock shelter sites is necessary in order to test the hypotheses advanced by Binford, Pettitt and Mellars, respectively. As demonstrated by this research, consideration of the nature of the occupations, as well as potential post-depositional disturbances must be incorporated into the analysis before the structure of spatial organization can be identified with confidence and interpreted. This is best conducted using three-dimensional analysis, assisted by the use of GIS. 


\section{- Chapter Eight - \\ Conclusions}

\section{1 - Introduction}

Palaeolithic archaeology provides important opportunities to study the behaviour of prehistoric populations. The spatial analysis of cave and rock shelter sites offer opportunities for identifying the types of activities that took place within them. In turn, this information can be used to test theories relating to the social and cognitive complexity of pre-modern hominids. Palaeolithic sites are often stratigraphically complex, representing a series of individual occupations. Therefore, the application of an appropriate, statistically based methodology is necessary. The results of this research prove the efficacy of combining Kintighs k-means cluster analysis and Geographic Information Systems.

\section{2 - Spatial Organization at Karabi Tamchin}

The k-means analysis of three archaeologically defined levels at the Middle Palaeolithic site of Karabi Tamchin (presented in Chapters 6 and 7) identified discrete, statistically significant clusters, which could be used to interpret spatial organization. Occupations at Karabi Tamchin were generally short-term and resulted in the deposition of a small number of artefacts. In archaeological levels II/2 and III, the hominid inhabitants used the outer edges of the shelter for the disposal of refuse, while the centre of the site was used for activities including bone processing and tool manufacture and/or rejuvenation. In level III, the presence of burnt bone materials associated with a bone processing area was identified on the basis of faunal materials identified. It was 
accompanied by significant numbers of tools (clusters 6-2 and 6-3, 6-cluster solution), which suggests that bone processing occurred in proximity to a hearth. Despite probable carnivore disturbance, it was still possible to distinguish activity areas based on differential artefact distributions in levels II/2 and III. Level IV could not be interpreted in terms of activity areas; instead, artefact distributions indicate the probable presence of palimpsests, demonstrating a small succession of at least two distinct, short-term occupations with statistically similar, vertically separated deposits.

\section{3 - Three-Dimensional Spatial Analysis}

One of the primary goals outlined at the beginning of this thesis was the implementation and evaluation of an effective method of three-dimensional spatial analysis. The SPSS v.10 and Kintigh's k-means programs were selected for quantitative analysis and combined with GIS for visual display. The results of the SPSS v.10 analysis produced clusters that are generally representative of the stratigraphic profile for the west wall; however, several important limitations associated with the procedure became apparent. First, the SPSS v.10 k-means cluster program requires the user to identify and define the number of clusters present in a dataset. This forces the data distribution to conform to a set number of clusters, without consideration of the natural clusters. While this is appropriate for some types of data, it means that for the purposes of this research, the program could only be used to verify the archaeological strata, but could not provide a true test of their statistical validity.

Second, an additional limitation of the SPSS v.10 k-means program concerns its application for datasets where stratigraphic separation between levels is poor. The 
program makes an assumption of equal variance in all three dimensions, which is invalid in many archaeological situations where limitations along the Z-axis are greater than limitations on either the X or Y-axis. At Karabi Tamchin, for example, lithological strata are very thin and the archaeological levels used in this research are not separated by thick, sterile deposits. The results of the SPSS v. 10 analysis show that complex stratigraphy influences the archaeologist's ability to distinguish natural vertical separations in the dataset. The SPSS k-means program can be used as a general check of the archaeological levels, but it is not an effective method for the analysis of complex stratigraphy.

Kintigh's (1994) Tools for Quantitative Archaeology offers several improvements over the SPSS v.10 program. While SPSS v.10 requires the user to define the number of clusters in the solution, Kintigh's (1994) program searches for statistically significant clusters in the dataset within a specified cluster range (e.g. 1 to 20 clusters). This process allows the program to identify optimal clustering solutions at various levels of resolution based on the natural distribution of archaeological materials. While the SPSS v.10 can be used where clusters are readily identifiable with the naked eye, Kintigh's program can be used in large, complex datasets where visual interpretation is difficult.

Kintigh's (1994) k-means analysis also improves the three-dimensional analysis of clusters through the application of a $Z$ expansion factor, creating a dataset with equal consideration in three dimensions. The $\mathrm{Z}$ expansion factor permits the identification of discrete clusters in the dataset, as well as vertical separations between clusters for the identification of successive occupations within palimpsest deposits, which is an important advantage over the SPSS v.10 program. 
The results of this research demonstrate that the flexibility of Kintigh's k-means analysis can provide an appropriate method for examining spatial structure in threedimensions. Through modification of the $\mathrm{Z}$ coordinates, it is possible to adapt the program to situations where archaeological deposits are difficult to interpret in the field, identifying discrete horizontal and vertical trends in the dataset. In the case of Karabi Tamchin, a $Z$ expansion factor of 10 proved effective.

\section{4 - K-means and Geographical Information Systems}

The results of the three-dimensional analysis support the use of the k-means cluster analysis in combination with Geographical Information Systems for the evaluation of three-dimensional data. ArcView 3.2 and ArcGIS were used in this research as adjuncts to the k-means analysis, providing an effective means of viewing cluster locations relative to one another and aiding in their interpretation. Many of the clusters identified by the $k$-means program could not be distinguished by simple visual inspection. Visualization can be used as an initial examination of the dataset; in situations where the vertical distribution of materials is complex, however subjective interpretations can be deceptive. The k-means program demonstrates its strength for the quantitative identification of cultural levels in a larger dataset, providing a method that is reliable and replicable. When this is combined with the impressive graphical capabilities of threedimensional GIS, it is possible to reproduce and interpret artefact distributions.

The incorporation of GIS into spatial analysis also facilitates the evaluation of intra-level distributions through complex data-handling capabilities. While artefact distributions can be analyzed using conventional paper maps, digital maps can be linked 
with a relational database that includes relevant attribute data (point location, artefact type, coordinates, etc.). This information can be used to identify and interpret various characteristics of the dataset, improving the ease and effectiveness of analysis and interpretations. While k-means analysis identifies statistically significant artefact concentrations, the visualization of cluster distributions in three dimensions is paramount to the discovery of distinct vertical trends.

\section{5 - Contributions to Archaeology}

The results of the level-by-level intra-site spatial analysis at Karabi Tamchin provides evidence that Neanderthals were making differential use of space for the performance of various activities within the shelter. The use of fire and the segregation of refuse and activity areas in the shelter are attested, contributing to the current debate concerning the relative cognitive complexity of prehistoric hominids. While researchers have suggested that it is difficult to discriminate Middle Palaeolithic hominid occupations from those of non-human carnivores (Mellars 1996; Pettitt 1997), this research provides evidence that a distinction is possible. The presence of burnt bone and stone tools within archaeological levels at Karabi Tamchin demonstrates a level of behavioural complexity that is distinguishable from non-human carnivores. As discussed in Chapters 2 and 7, comparisons between non-human carnivores and Neanderthals must consider hearthrelated assemblages, which cannot be attributed to occupation by non-human carnivores (Vaquero 1999; Vaquero and Pasto 2001).

Researchers have suggested that spatial patterning within Neanderthal occupations is the result of pragmatic responses to the environment and does not 
demonstrate cognitive complexity (Mellars 1996). At Karabi Tamchin, activity areas are located centrally, while refuse areas tend to be located near the edges of the shelter. While these activities can be distinguished from non-human carnivores, their arrangement supports pragmatic use of space, which is comparable to modern human behaviour. While it is possible to differentiate between non-human carnivore and Neanderthal spatial organization, the results of this research cannot be used to interpret cognitive complexity. Further investigation and interpretation are necessary to contribute to current hypotheses regarding the use space in Middle Palaeolithic populations.

Finally, the spatial complexity associated with Palaeolithic occupations, resulting from processes such as post-depositional disturbances and the presence of palimpsests, suggests that continued investigation and methodological refinement are necessary. This research demonstrates that the combined application of k-means and GIS is an innovative step towards such a methodological refinement. Spatial analysis in archaeology has generally been limited to investigations of two-dimensional horizontal distributions, or the incorporation of a third dimension into two-dimensional interpretations. The distribution of archaeological materials at Karabi Tamchin illustrates the importance of true, three-dimensional investigations, which can reveal phenomena in the data that cannot be identified through two-dimensional analysis. The successful application of GIS highlights its potential for archaeological applications. While it has generally been restricted to predictive modelling, continued, varied applications will make GIS a powerful analytical tool for archaeological research. 


\section{6 - Future Recommendations}

As demonstrated by the research at Karabi Tamchin, spatial analysis has the potential to reveal information about the use of space in archaeological sites. Despite successful application, the three-dimensional dataset used in this research was a limited sample of the total distribution of archaeological materials, prohibiting the investigation of archaeological level V/2. The recording and recovery of a larger three-dimensional dataset would improve the results of the analysis, strengthening the identification and interpretation of activity areas. Prior to excavation, archaeologists need to identify whether spatial analysis will be part of the investigation, in order to develop a suitable strategy for mapping features and interpreting the stratigraphic profile. Linking threedimensionally recorded artefacts with individually numbered tools and faunal specimens would also be desirable. This information can be incorporated into a GIS database to refine the analysis and develop a better understanding of the activities taking place within the archaeological levels.

In this research, GIS is used specifically for the visualization and database query of horizontal and vertical distributions, while statistical analysis was carried out using additional methods. Current GIS are limited in their ability to analyze three-dimensional datasets. Future improvements of GIS software should involve further developments for three-dimensional analysis, including statistical analysis. This will permit archaeologists to perform their analyses within one program, as the transfer of data between several programs is time-consuming and can introduce error.

Through continued excavation of Middle Palaeolithic sites in the Crimea, it will be possible to develop a regional interpretation of spatial organization and the nature of 
occupations within cave and rock shelter sites. The development and refinement of analytical techniques, including improved three-dimensional analysis, will lead to improvements in the interpretation of materials. 
- Appendix A -

Frequency Distribution of Total Faunal Materials

Recovered from Karabi Tamchin 


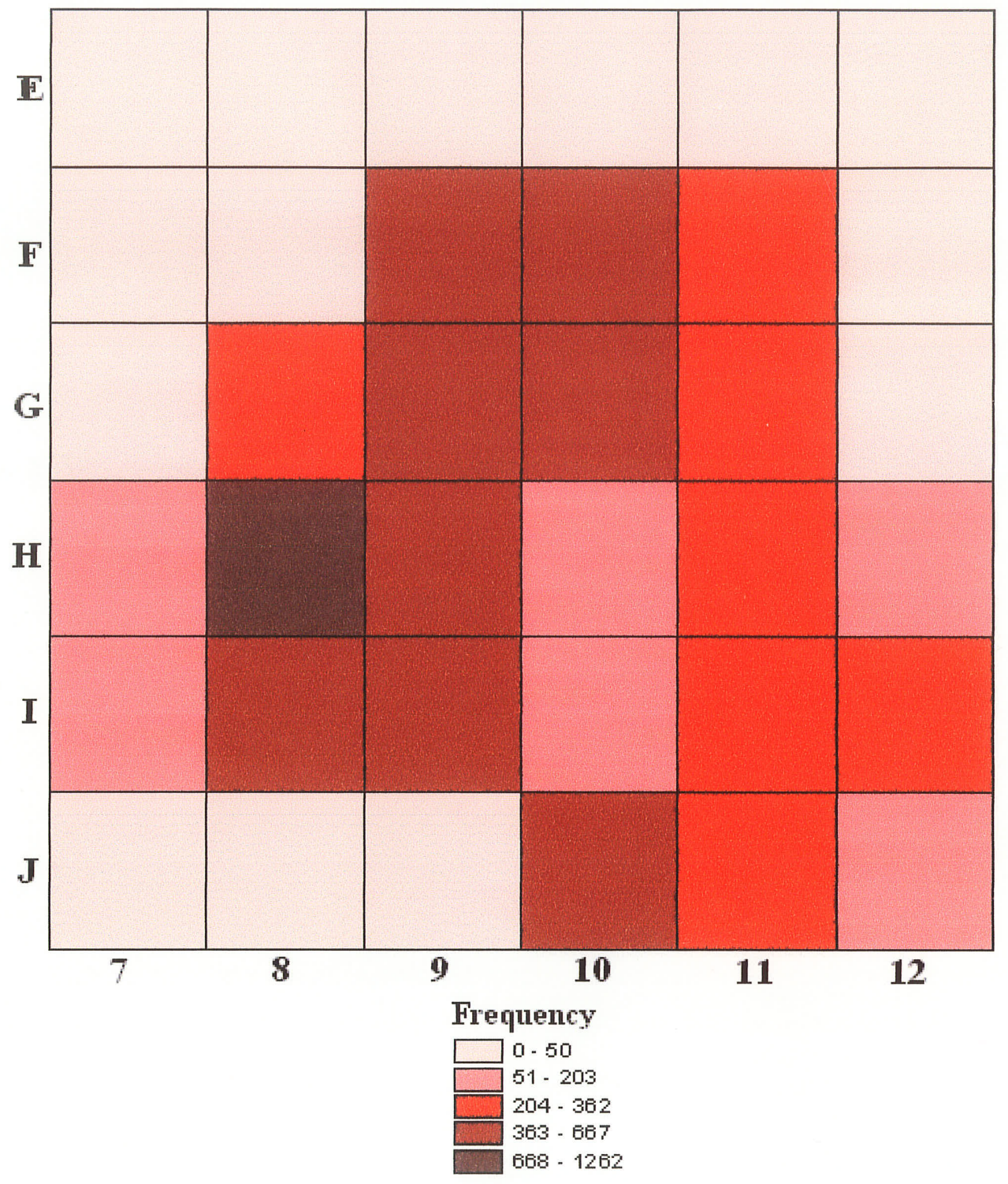

Figure 1: Total Bone Distribution, Level II 


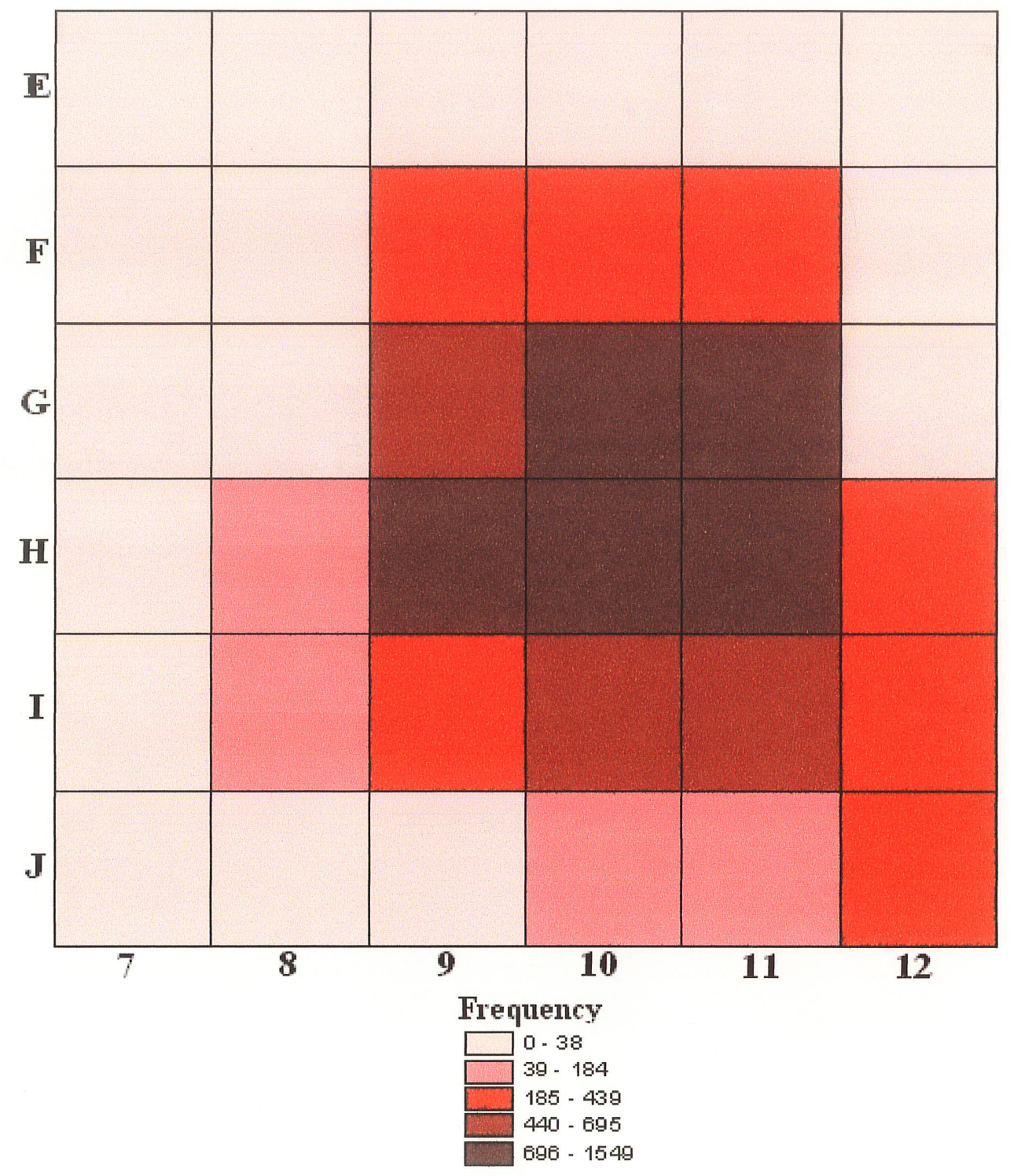

Figure 2: Total Bone Distribution, Level III 


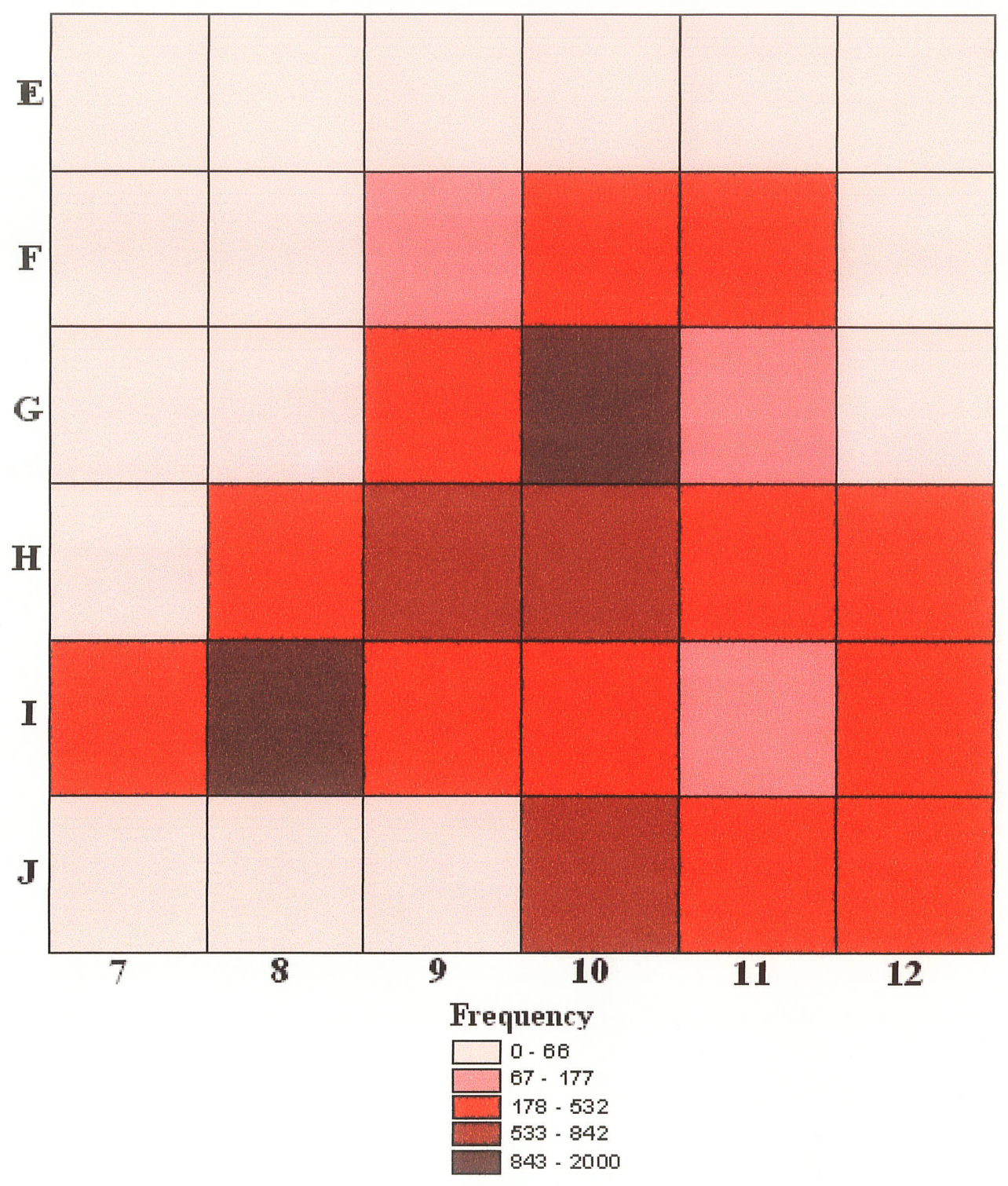

Figure 3: Total Bone Distribution, Level IV 


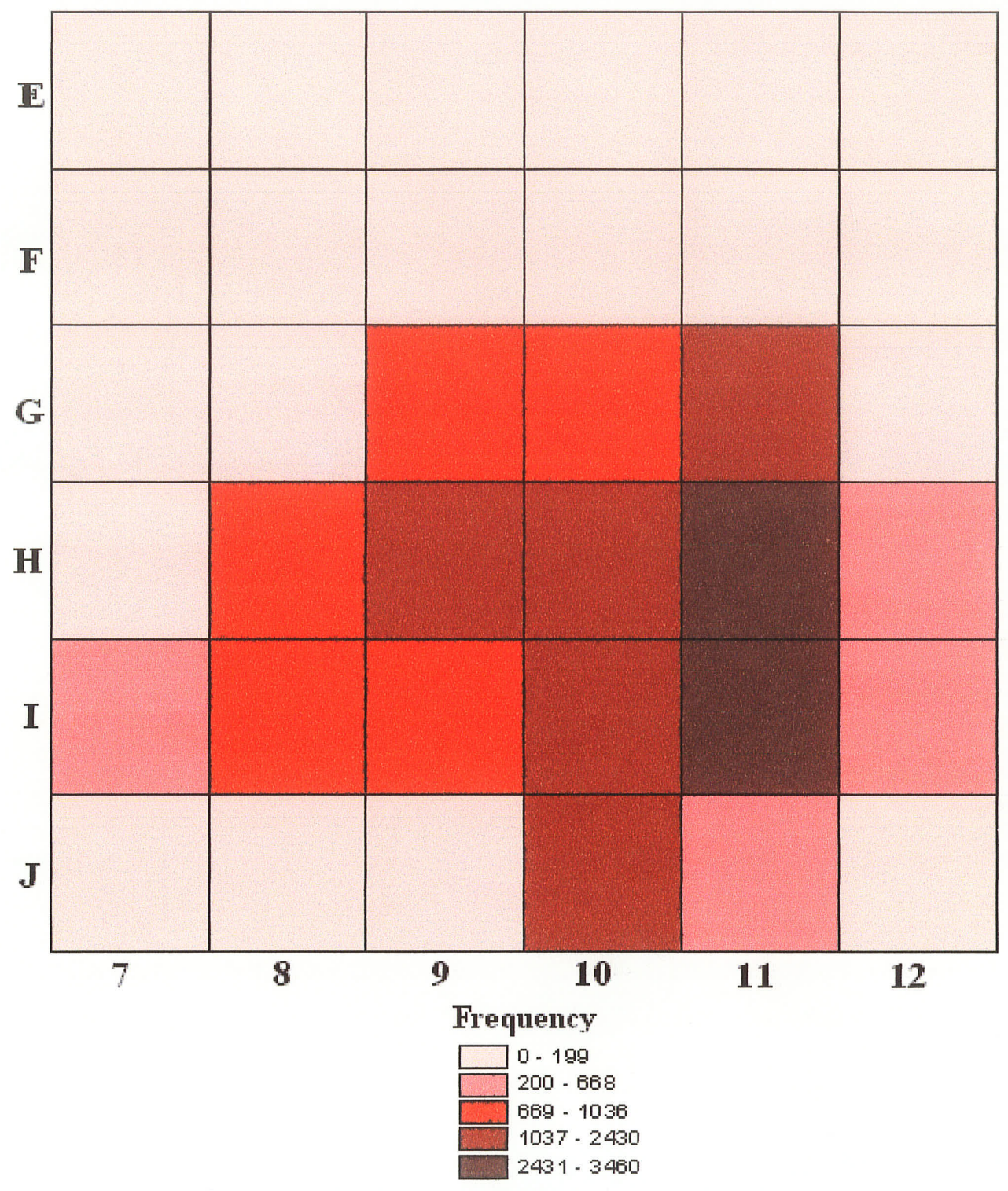

Figure 4: Total Bone Distribution, Level V/2 


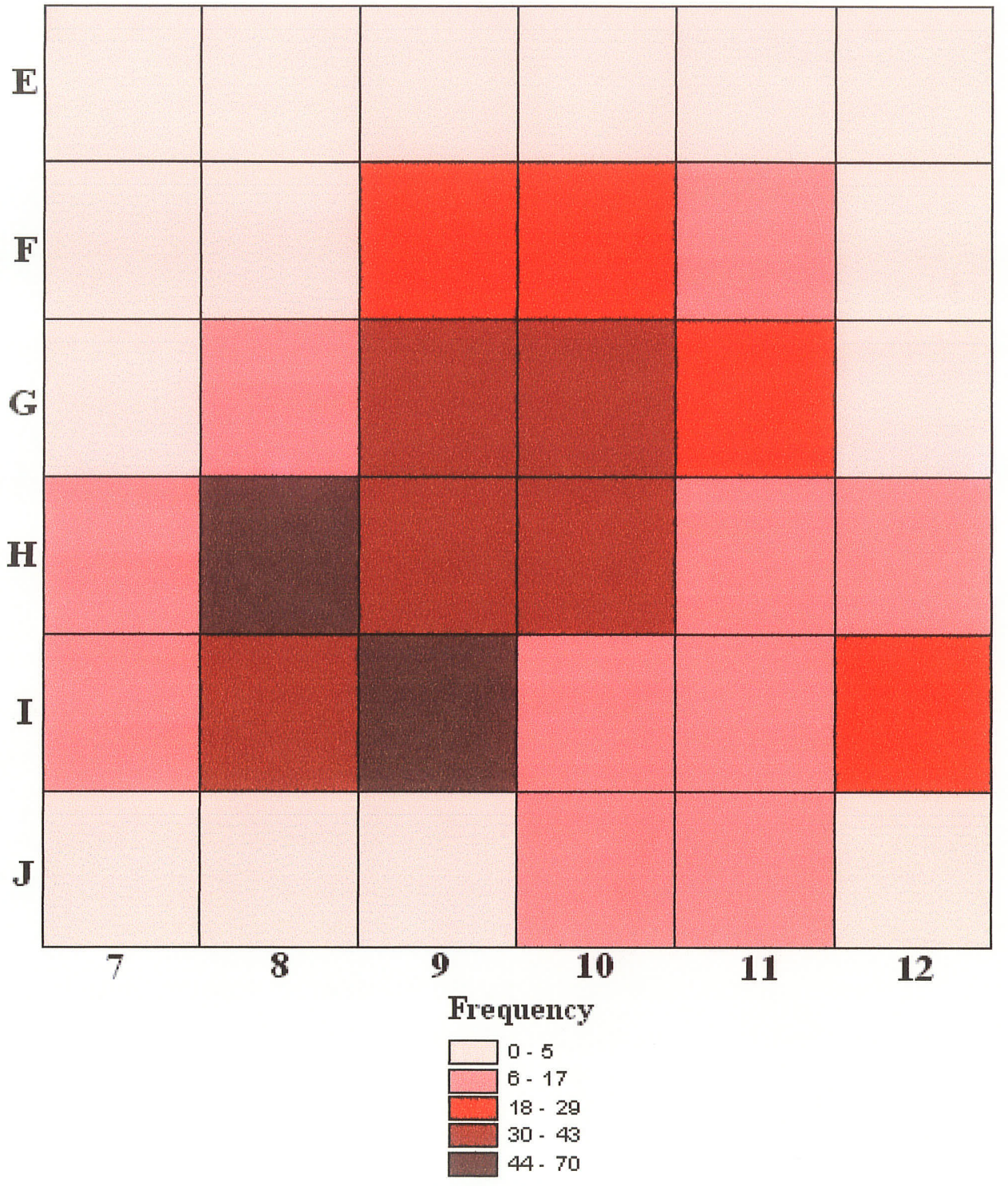

Figure 5: Total Identified Bone Distribution, Level II 


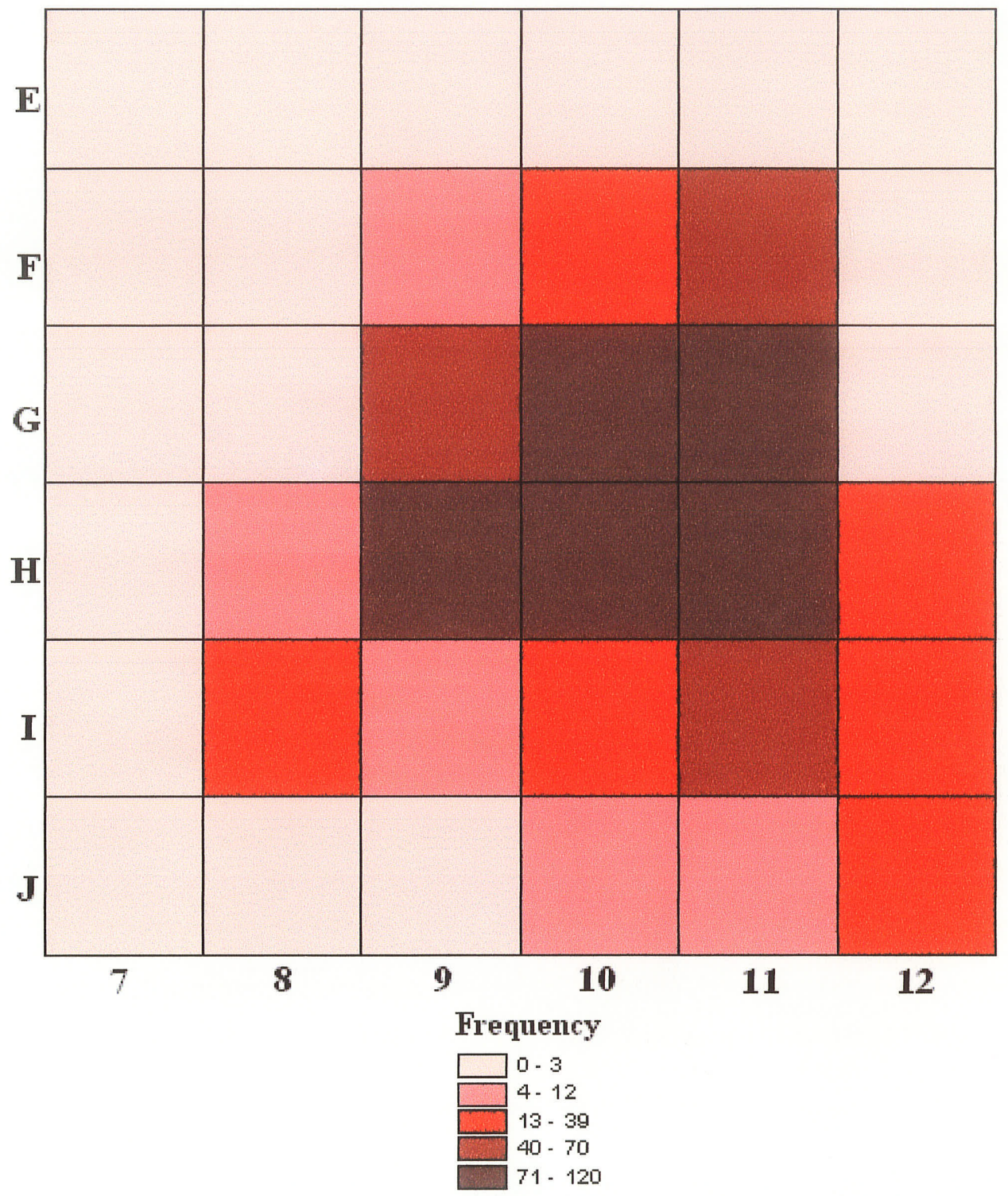

Figure 6: Total Identified Bone Distribution, Level III 


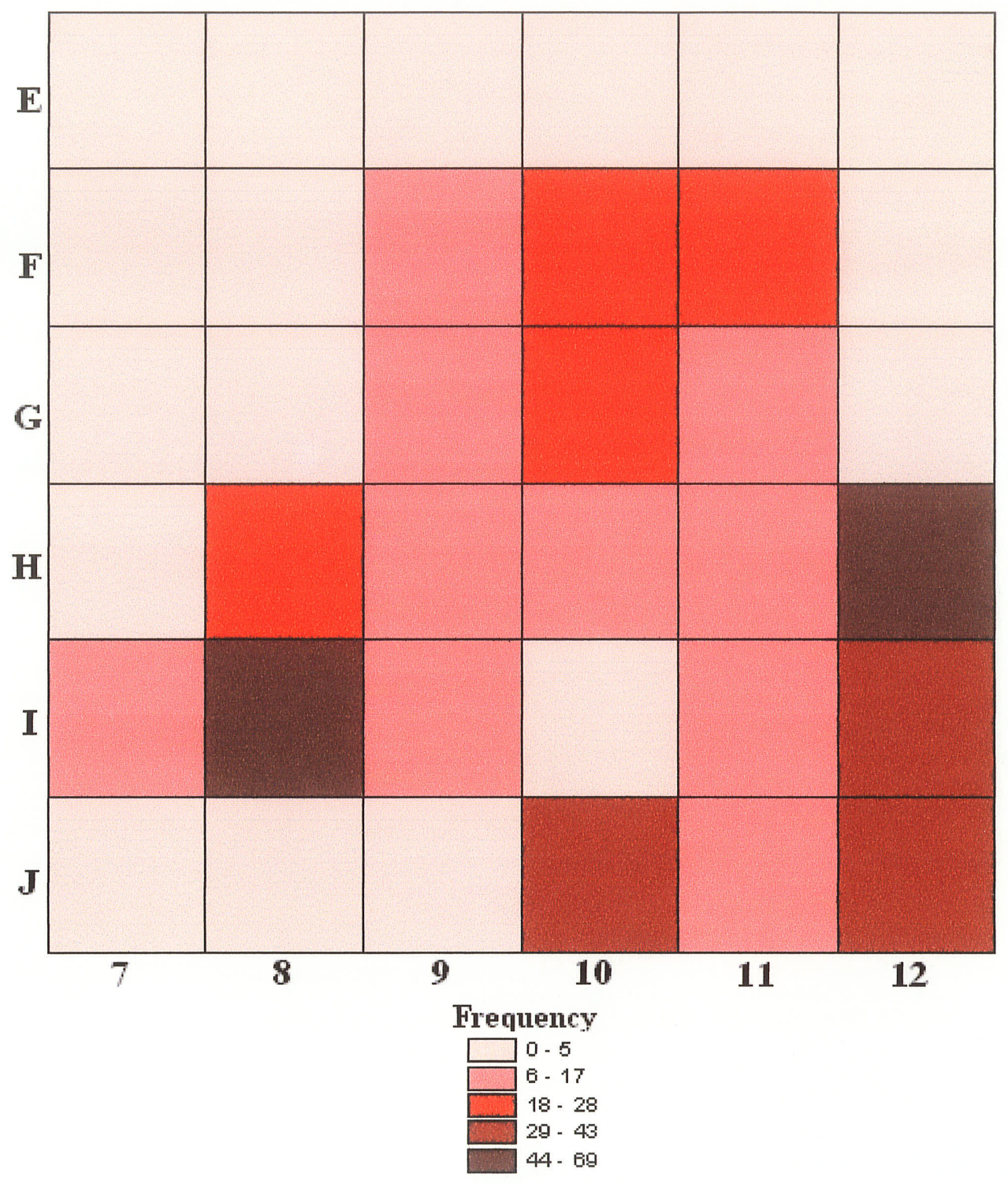

Figure 7: Total Identified Bone Distribution, Level IV 


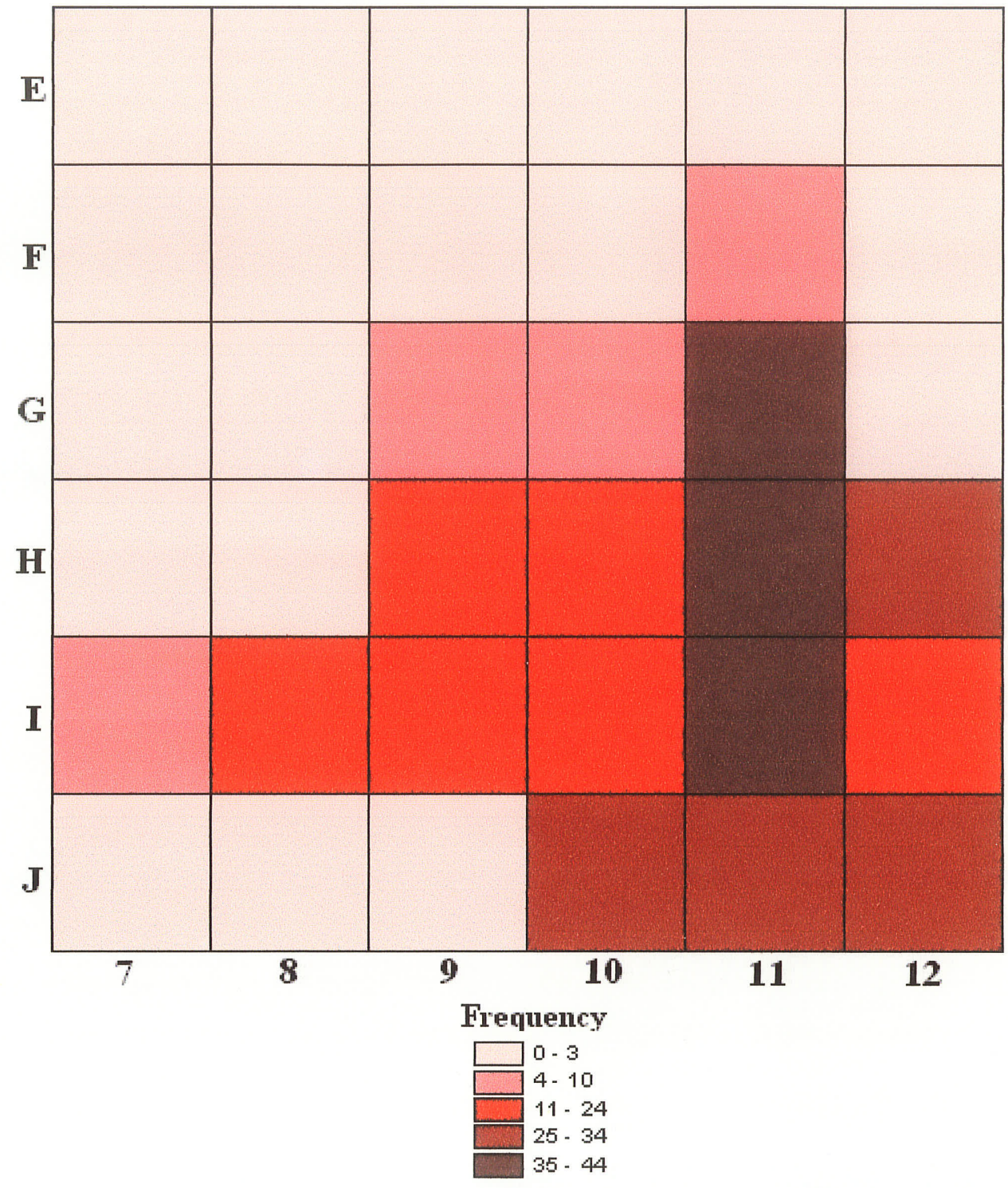

Figure 8: Total Identified Bone Distribution, Level V/2 


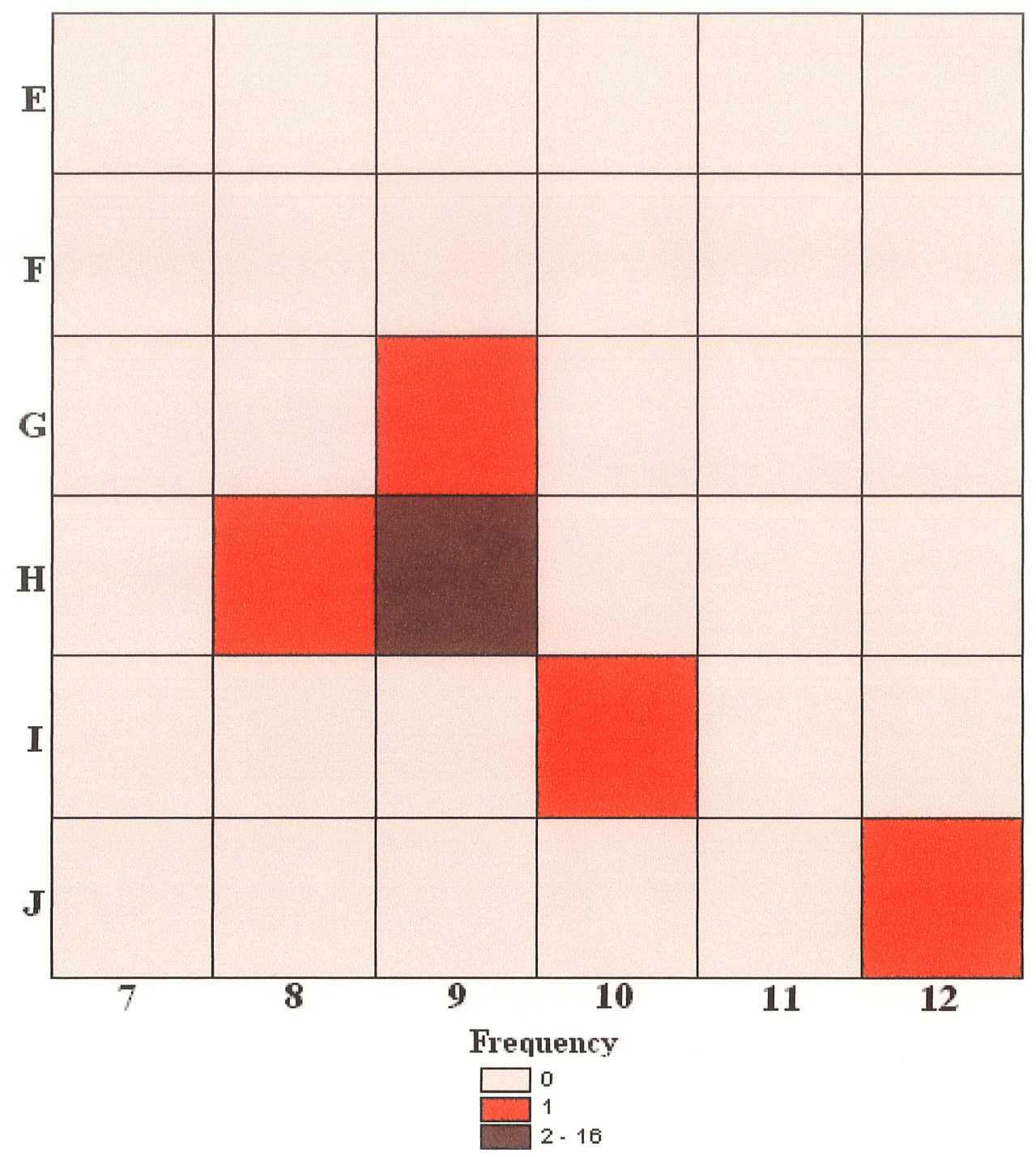

Figure 9: Total Burnt Bone Distribution, Level III 


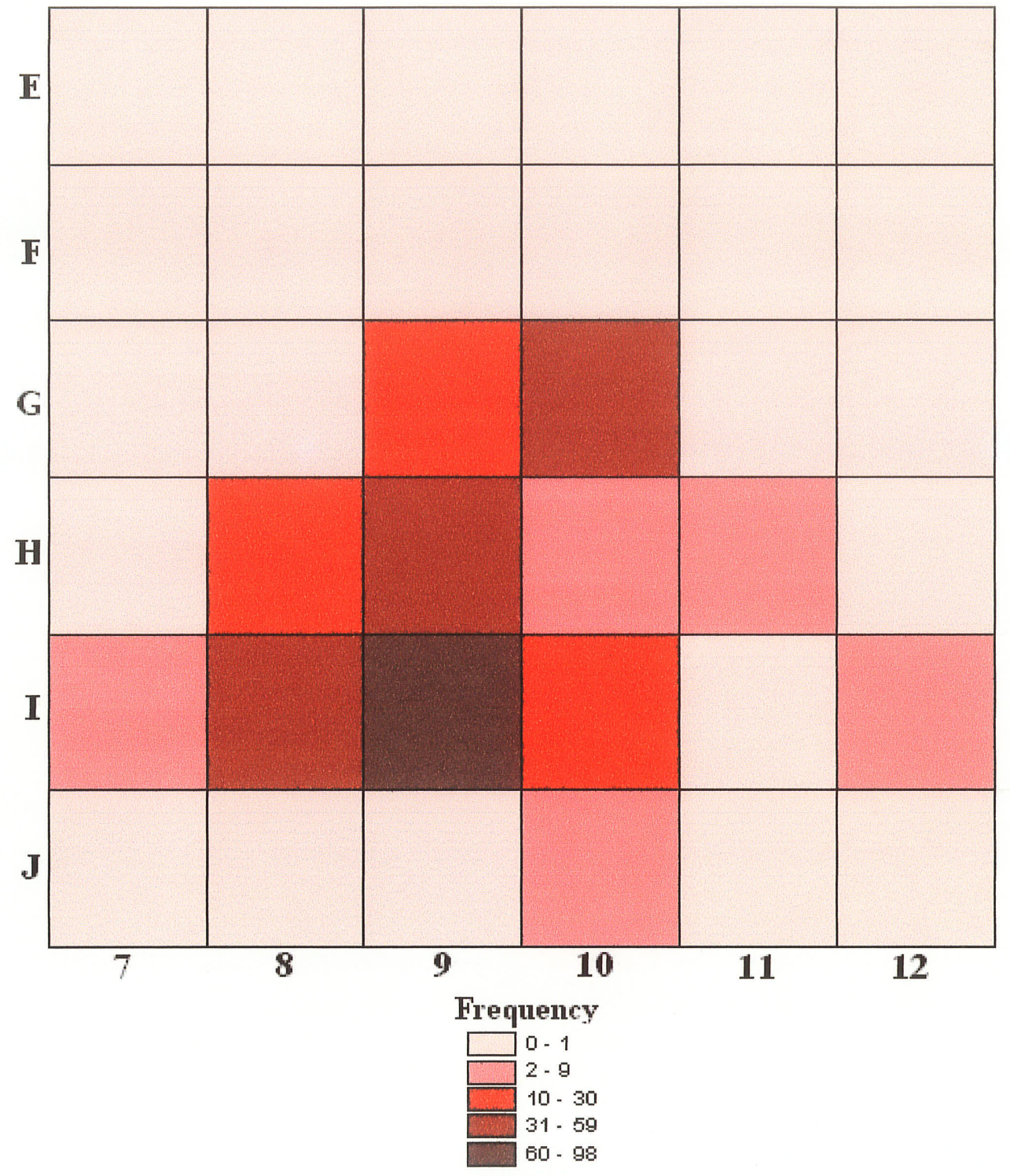

Figure 10: Total Burnt Bone Distribution, Level IV 


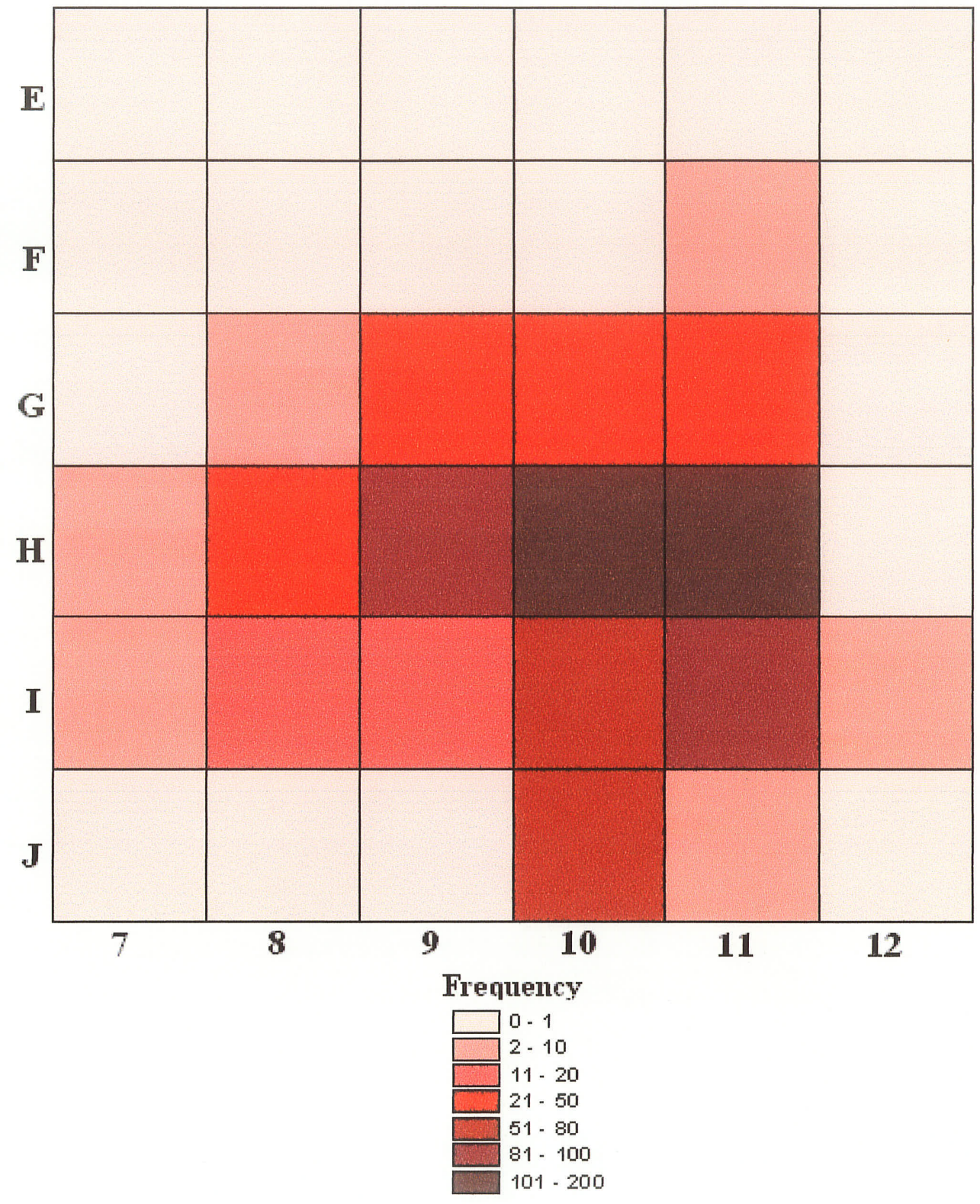

Figure 11: Total Burnt Bone Distribution, Level V/2 


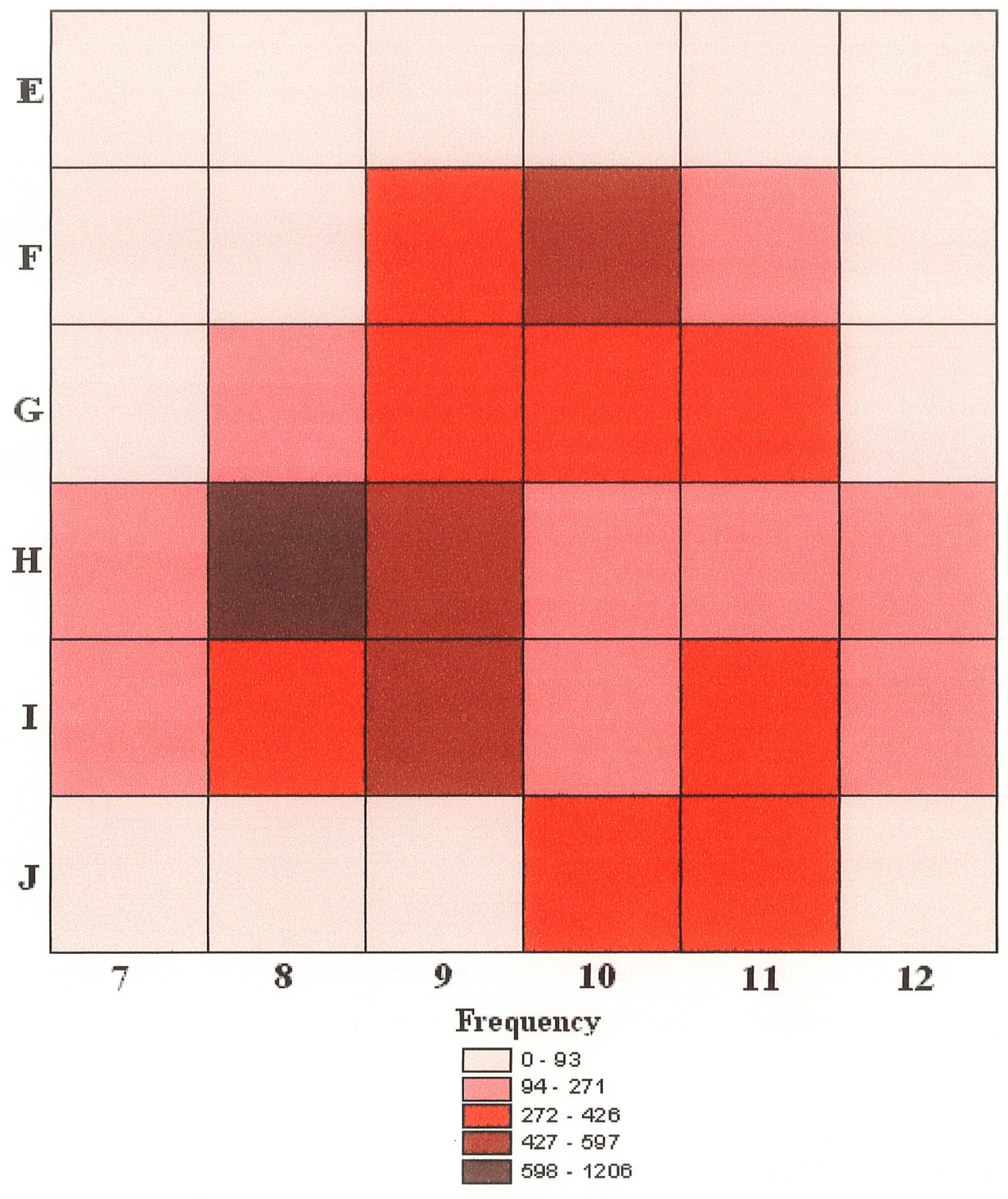

Figure 12: Total Unidentified Bone Distribution, Level II 


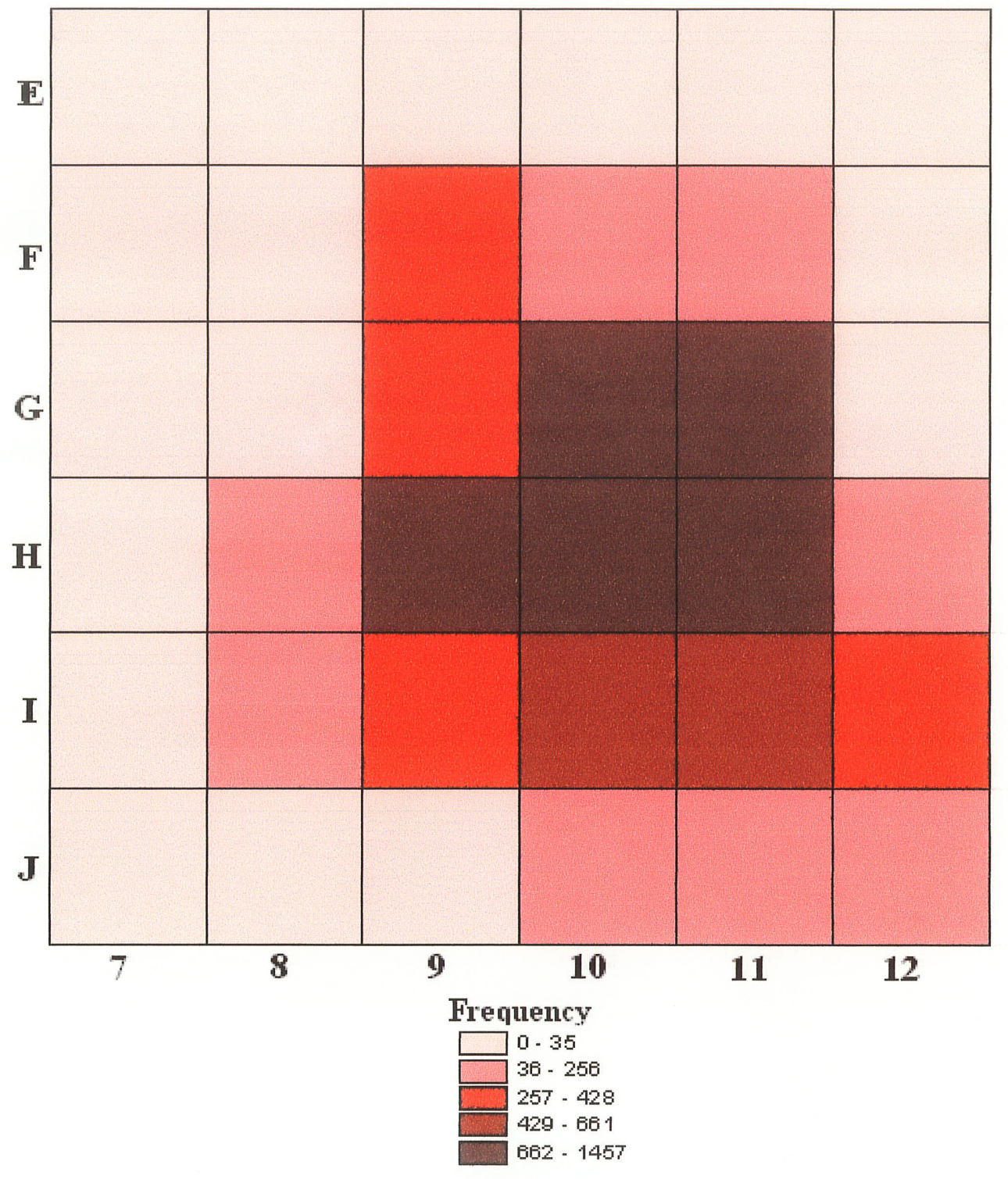

Figure 13: Total Unidentified Bone Distribution, Level III 


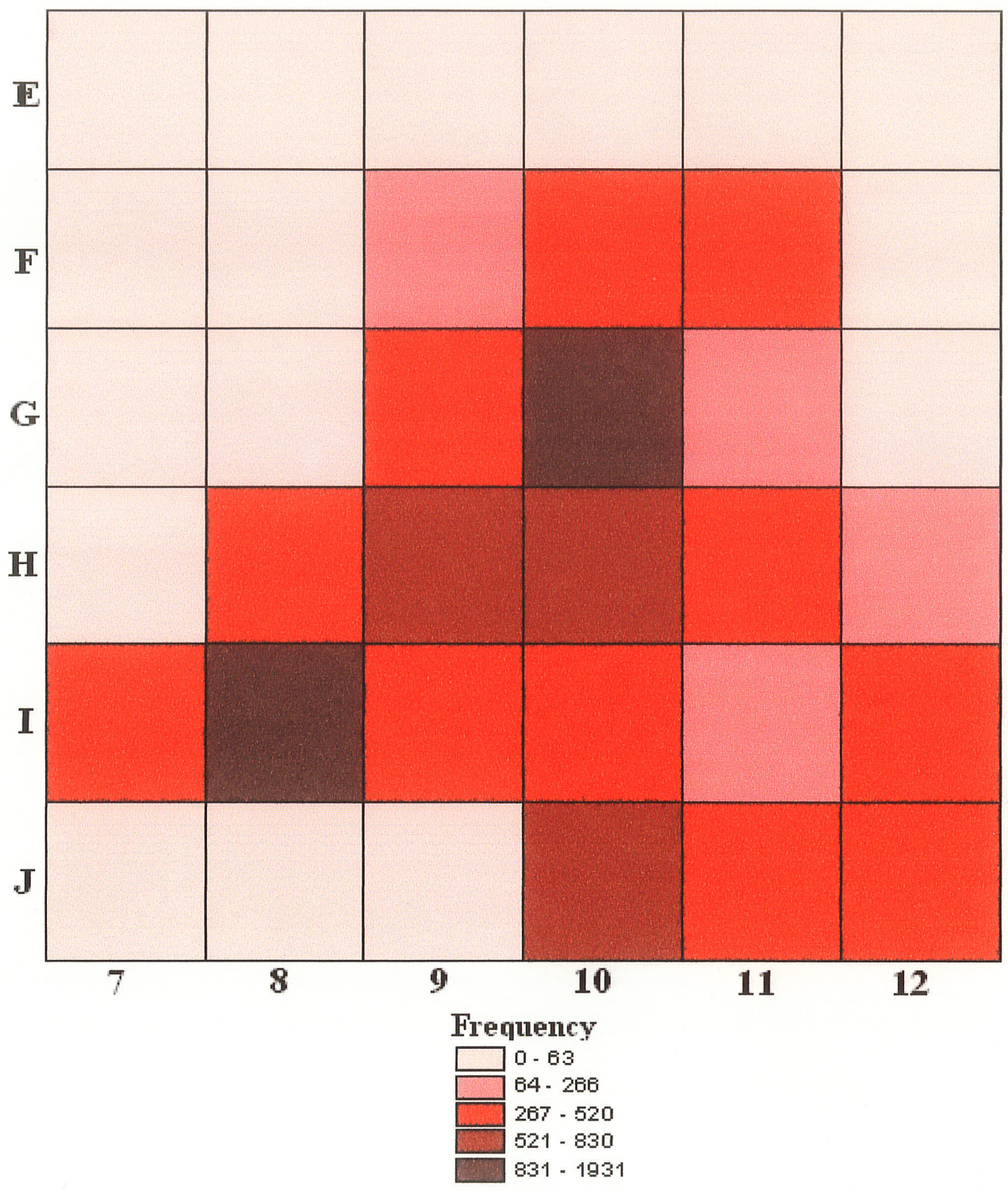

Figure 14: Total Unidentified Bone Distribution, Level IV 


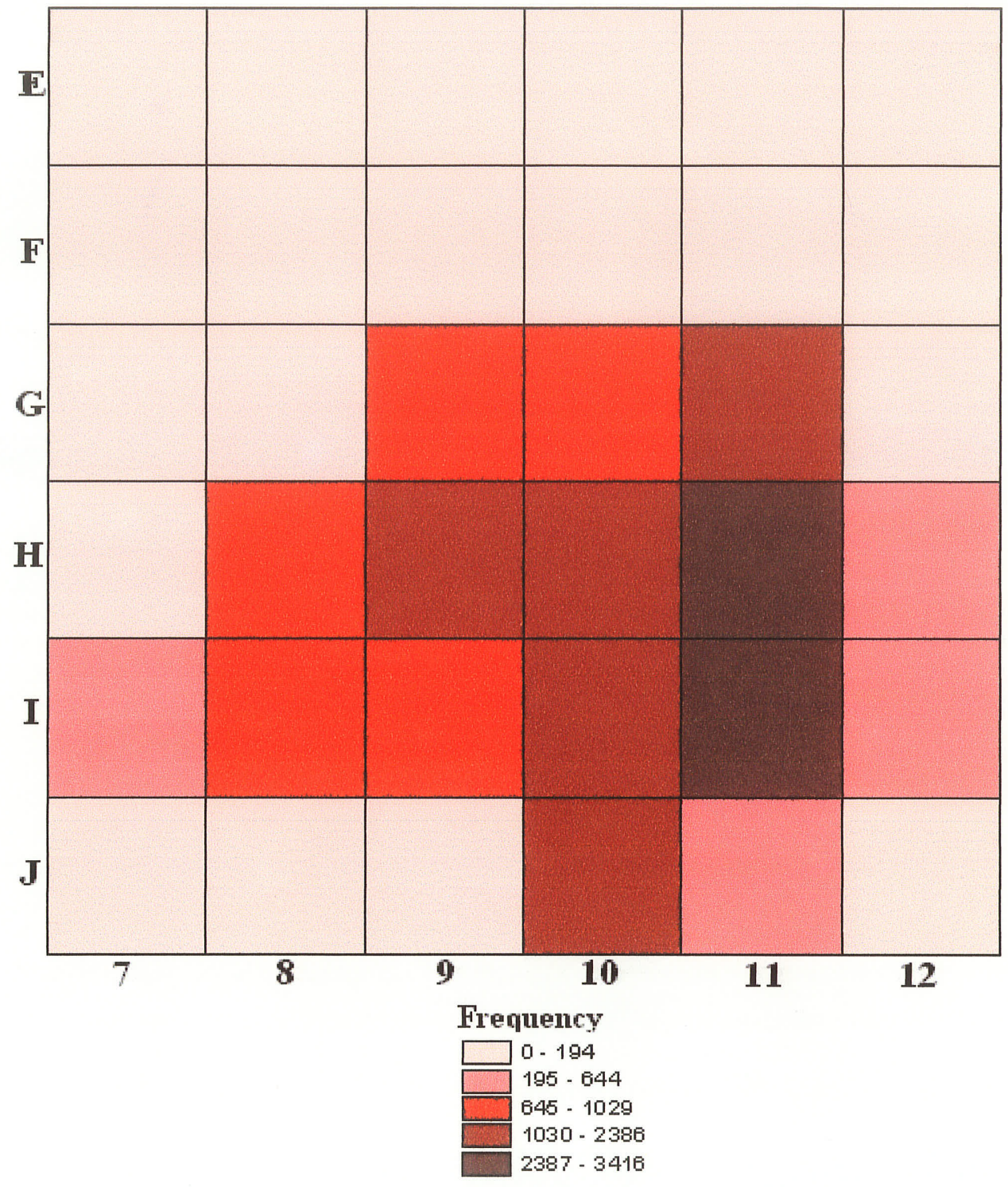

Figure 15: Total Unidentified Bone Distribution, Level V/2 
- Appendix B -

Horizontal Artefact Distributions

Re-Sampled Levels 


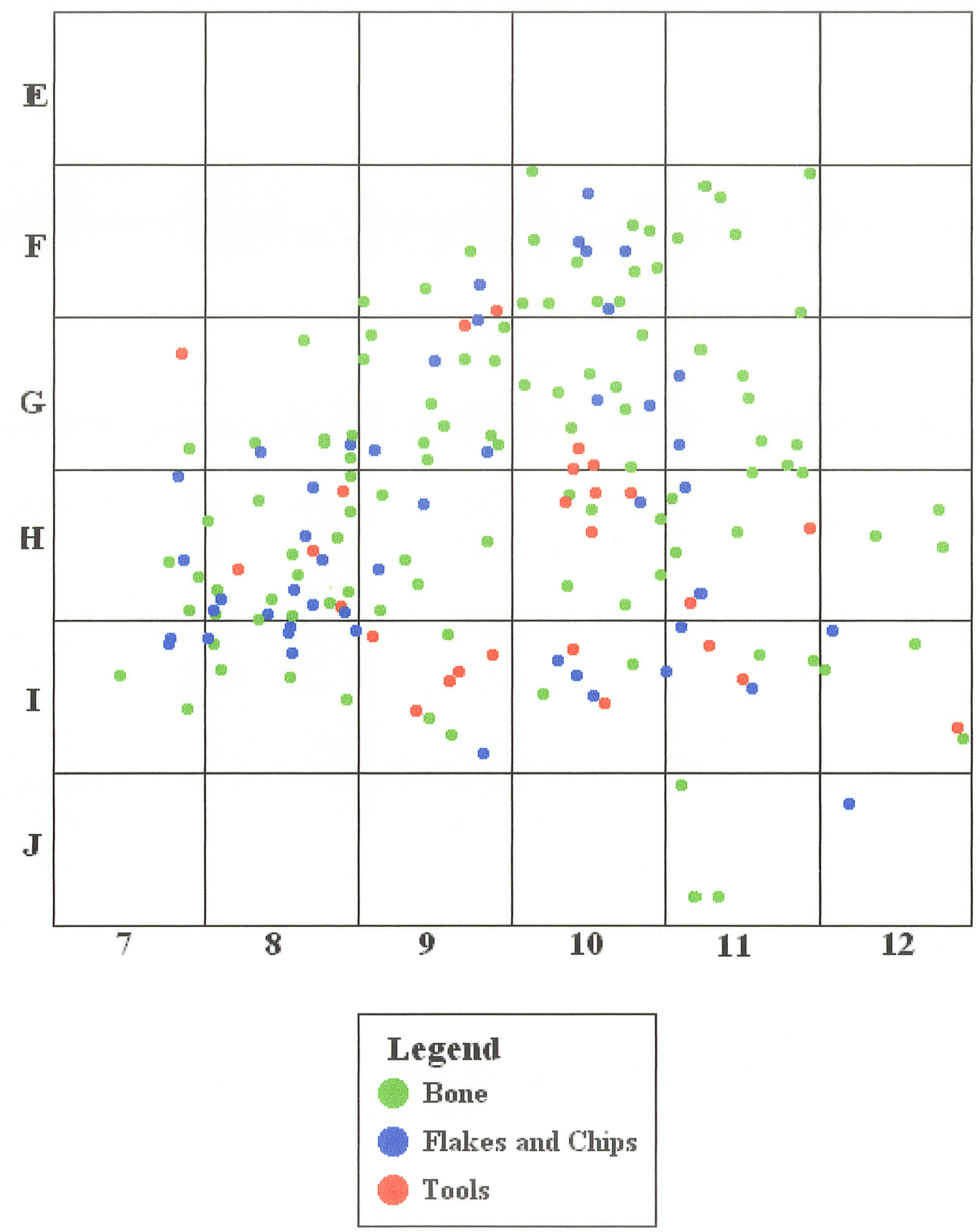

Figure 1: Total Artefact Distribution, Re-Sampled Dataset - Level II/2 


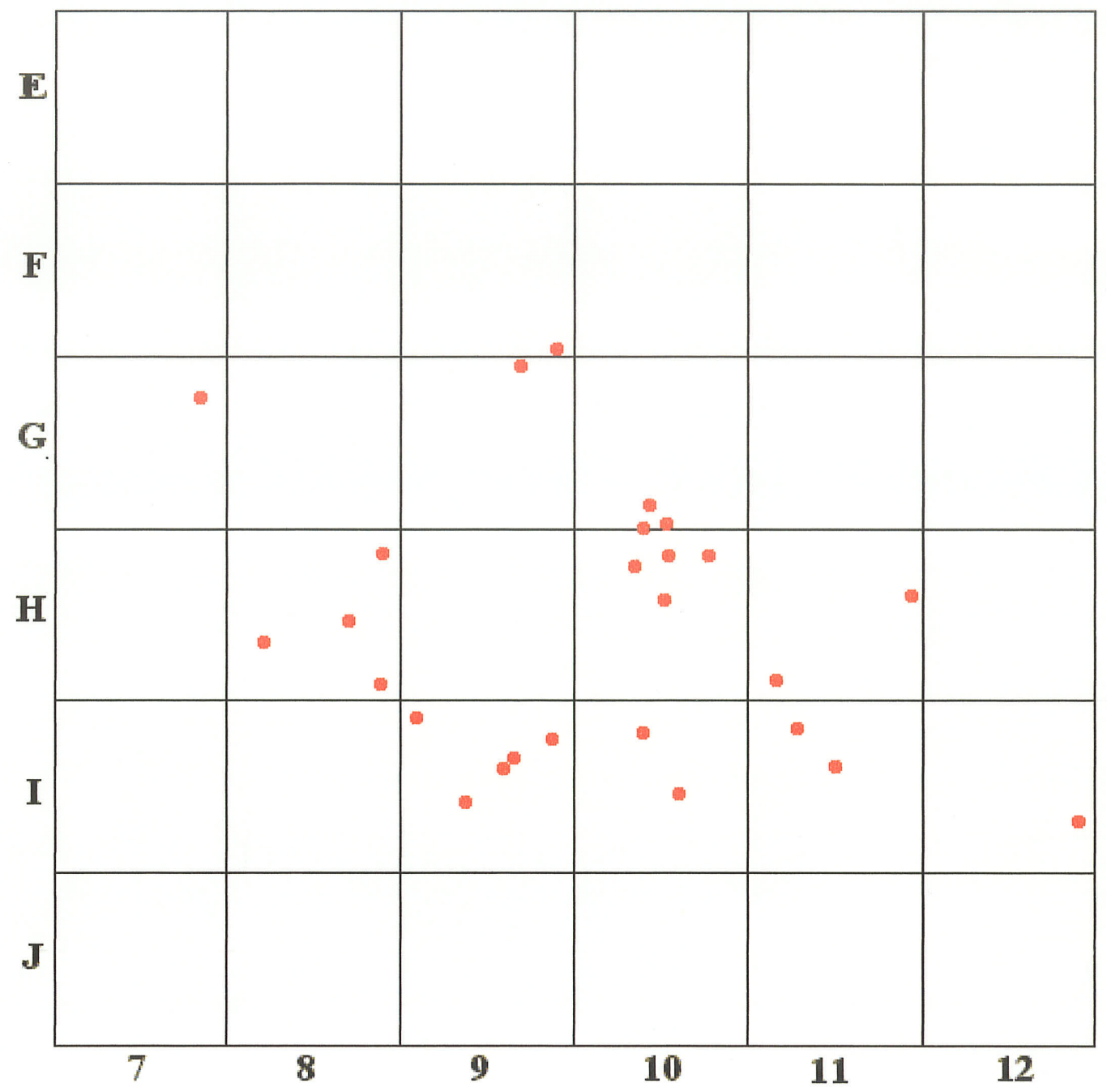

Figure 2: Stone Tool Distribution, Re-Sampled Dataset - Level II/2 


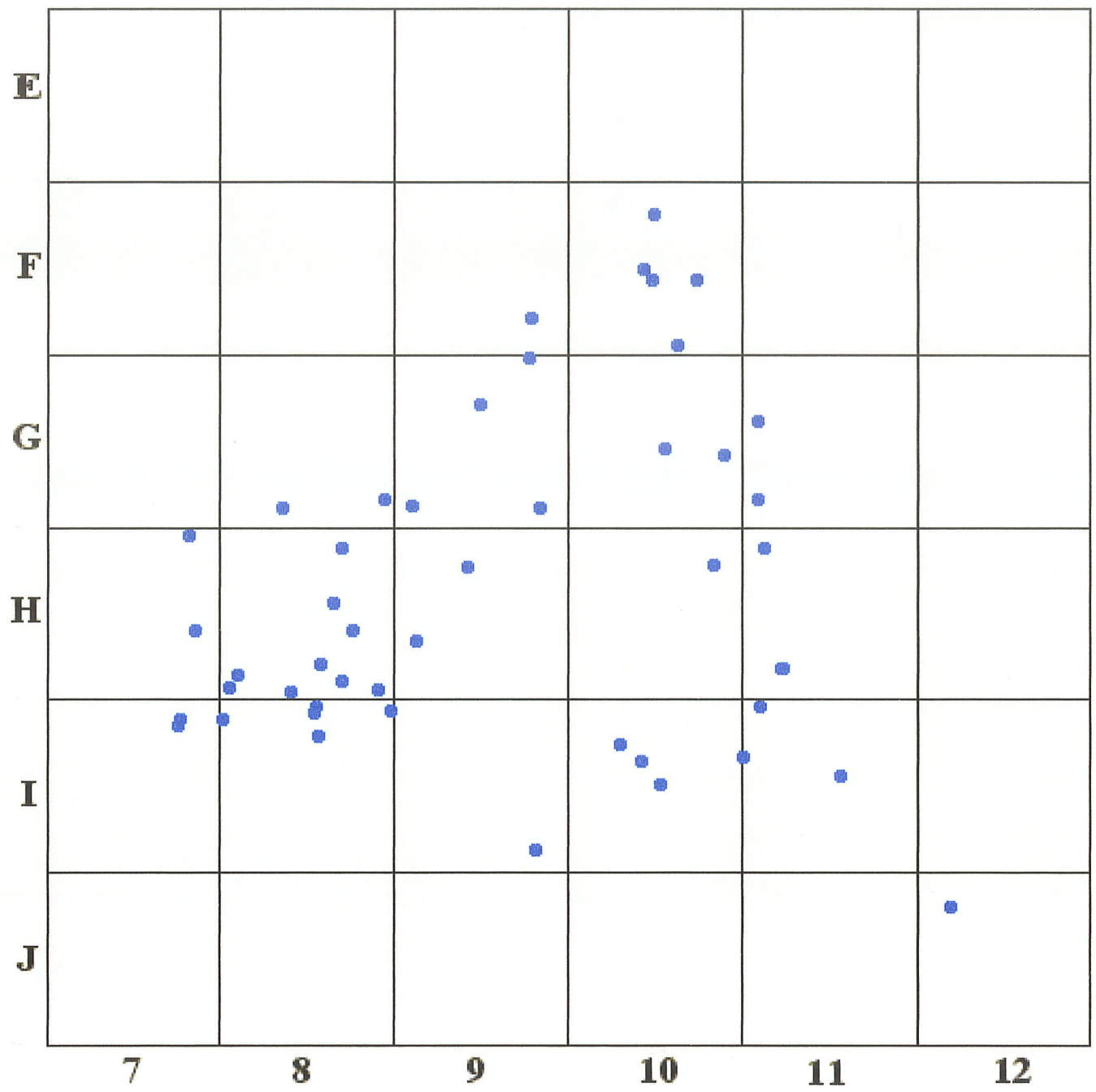

Figure 3: Stone Flakes and Chips Distribution, Re-Sampled Dataset - Level II/2 


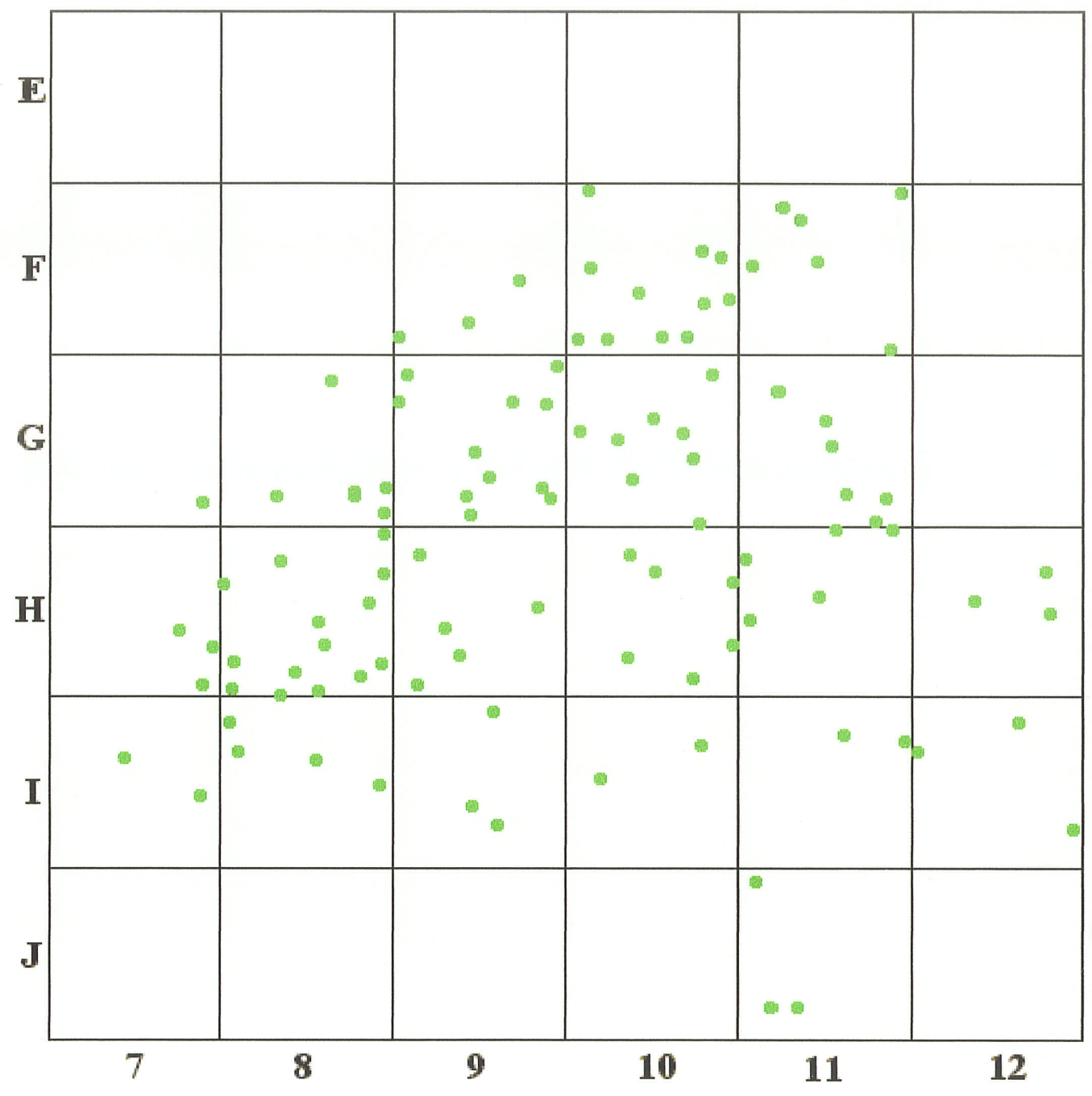

Figure 4: Bone Distribution, Re-Sampled Dataset - Level II/2 


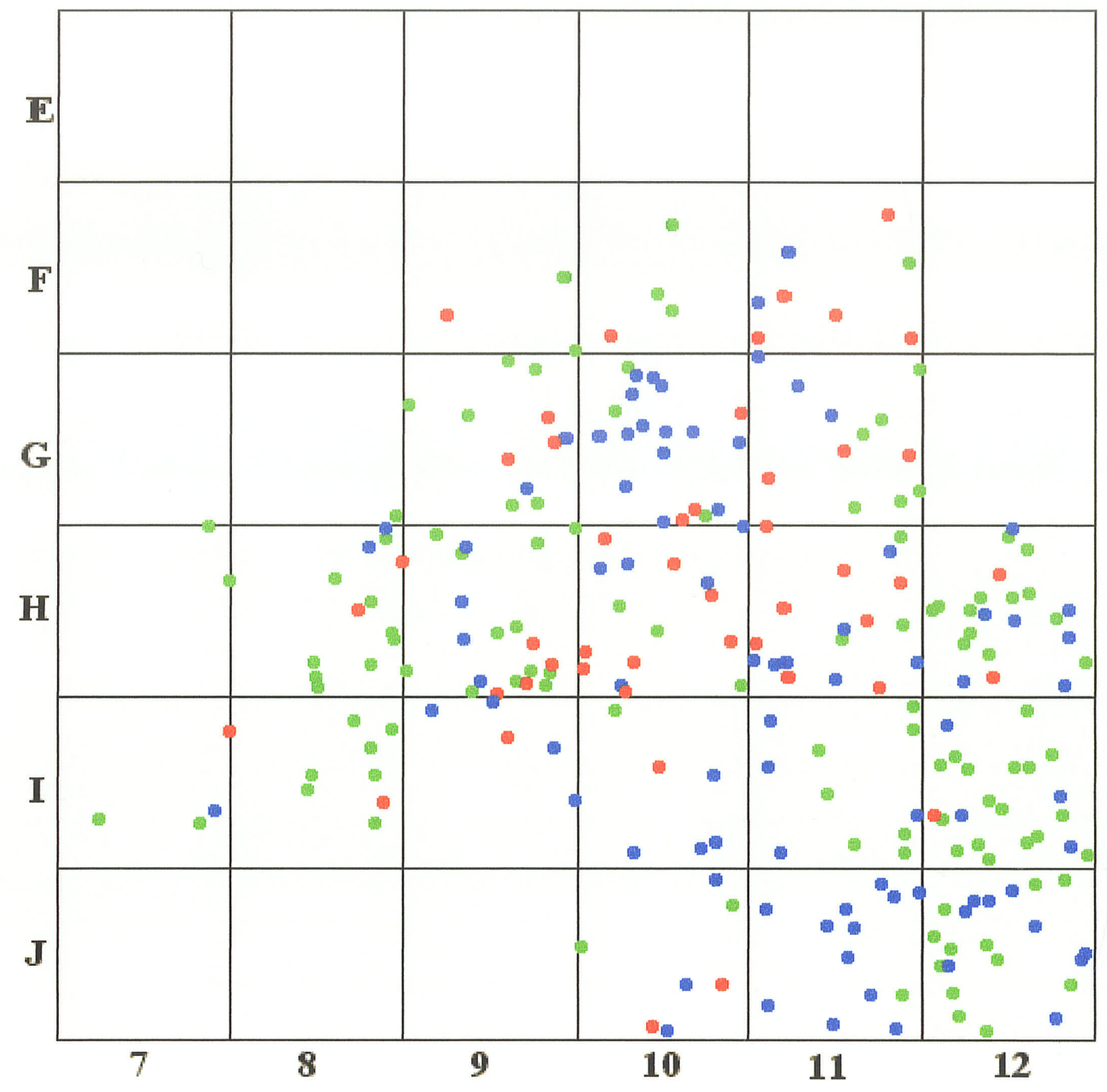

\section{Legend \\ Bone \\ - Flakes and Chips \\ - Tools}

Figure 5: Total Artefact Distribution, Re-Sampled Dataset - Level III 


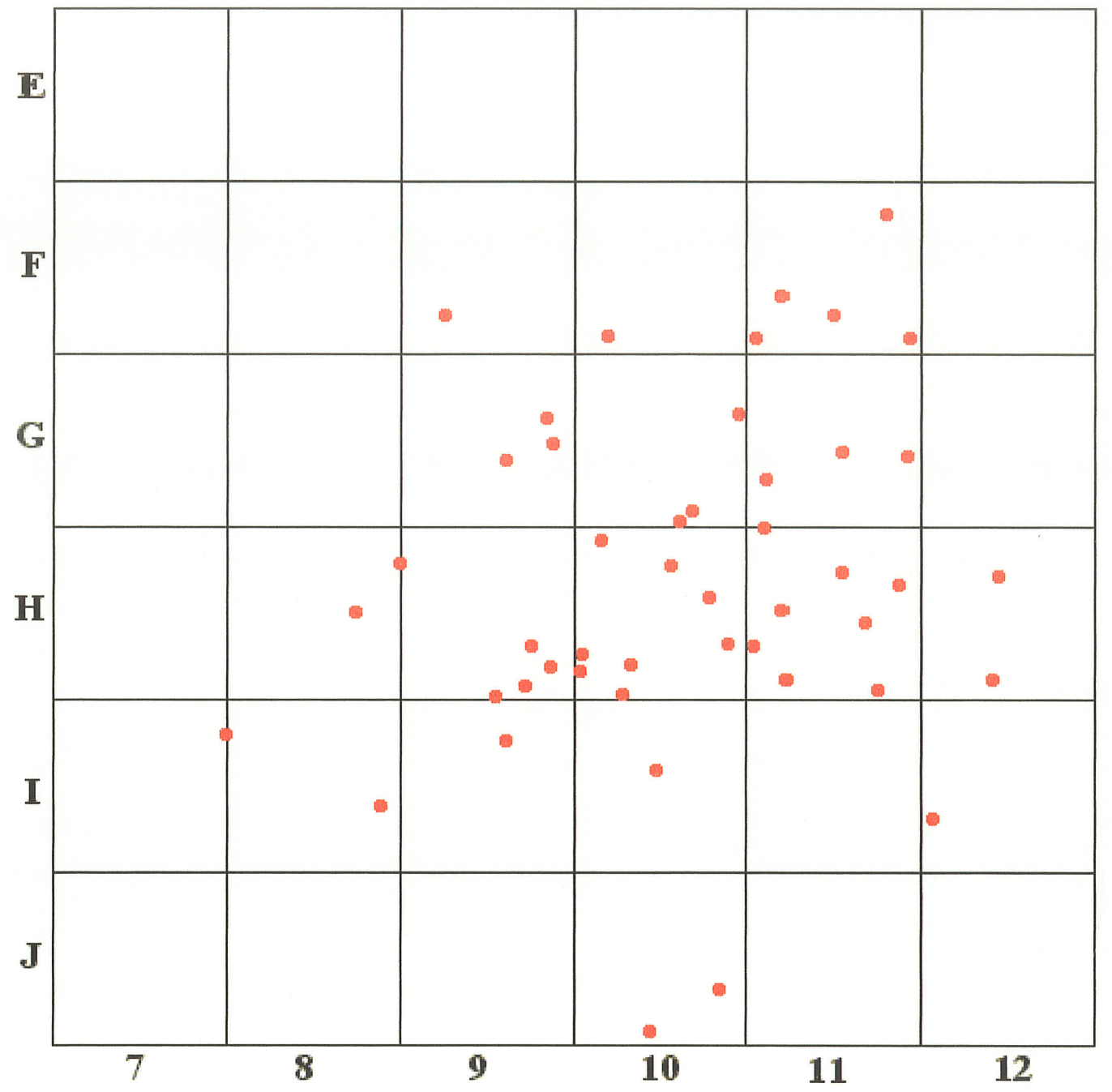

Figure 6: Stone Tool Distribution, Re-Sampled Dataset - Level III 


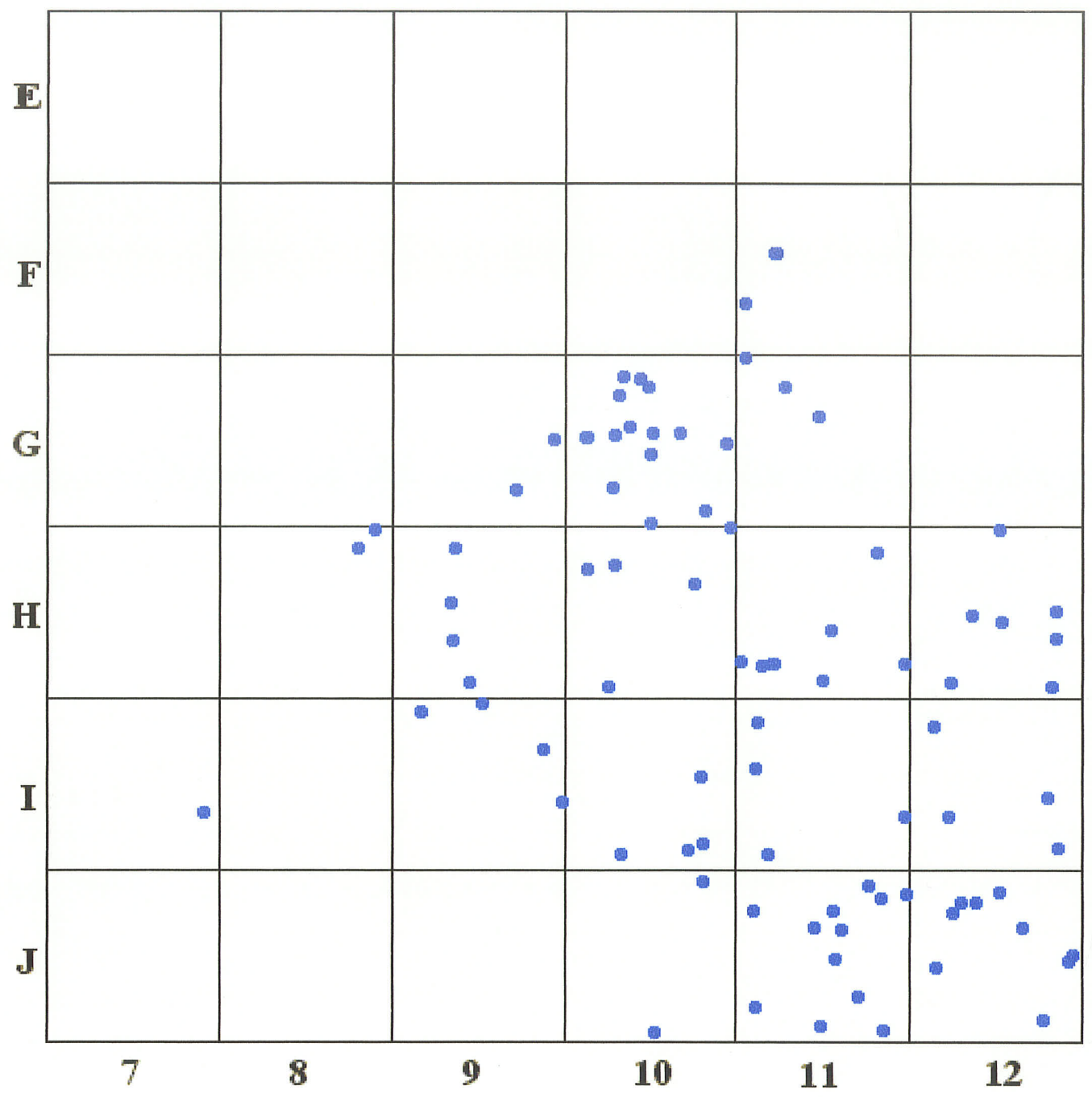

Figure 7: Stone Flakes and Chips Distribution, Re-Sampled Dataset - Level III 


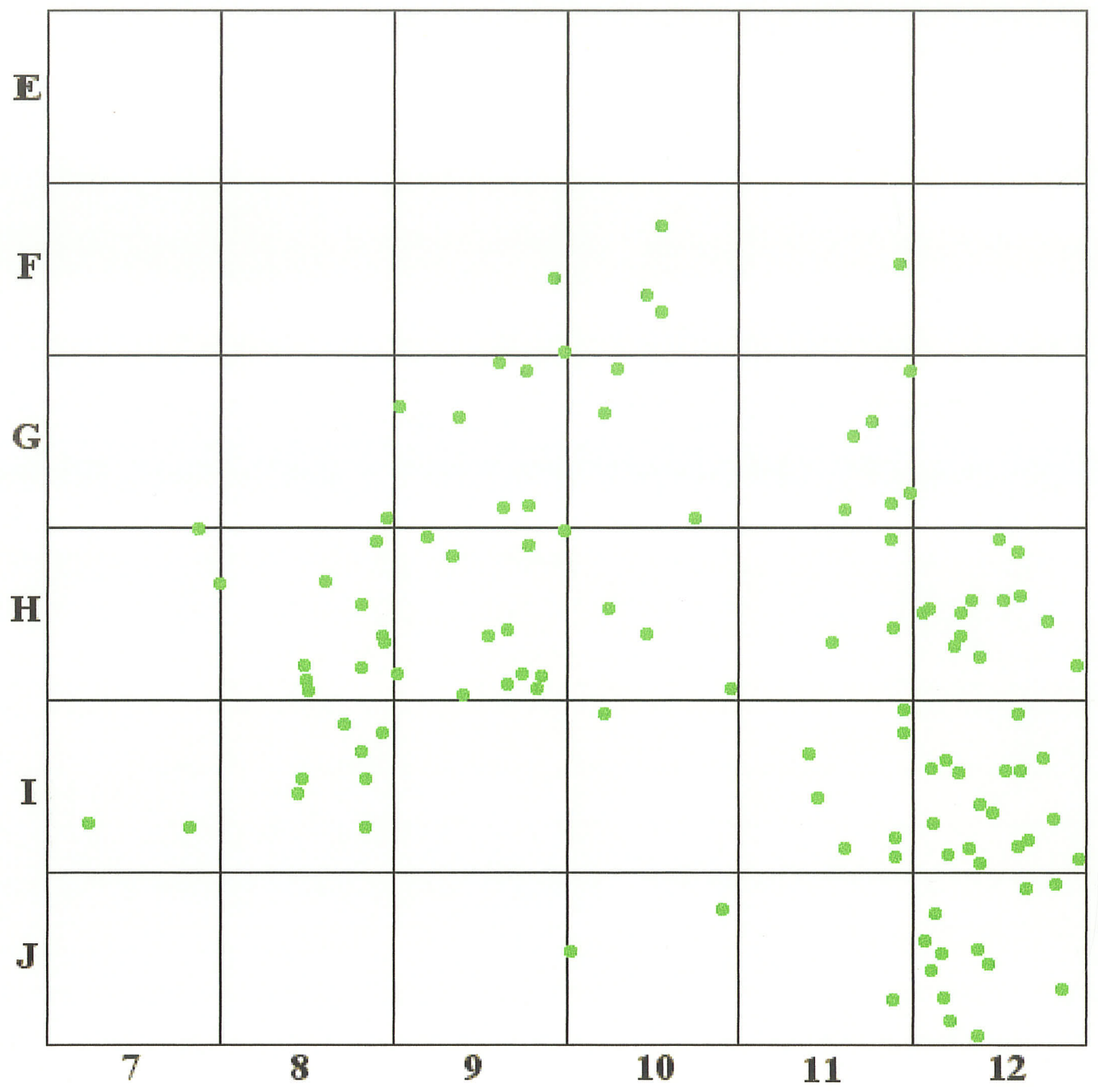

Figure 8: Bone Distribution, Re-Sampled Dataset - Level III 


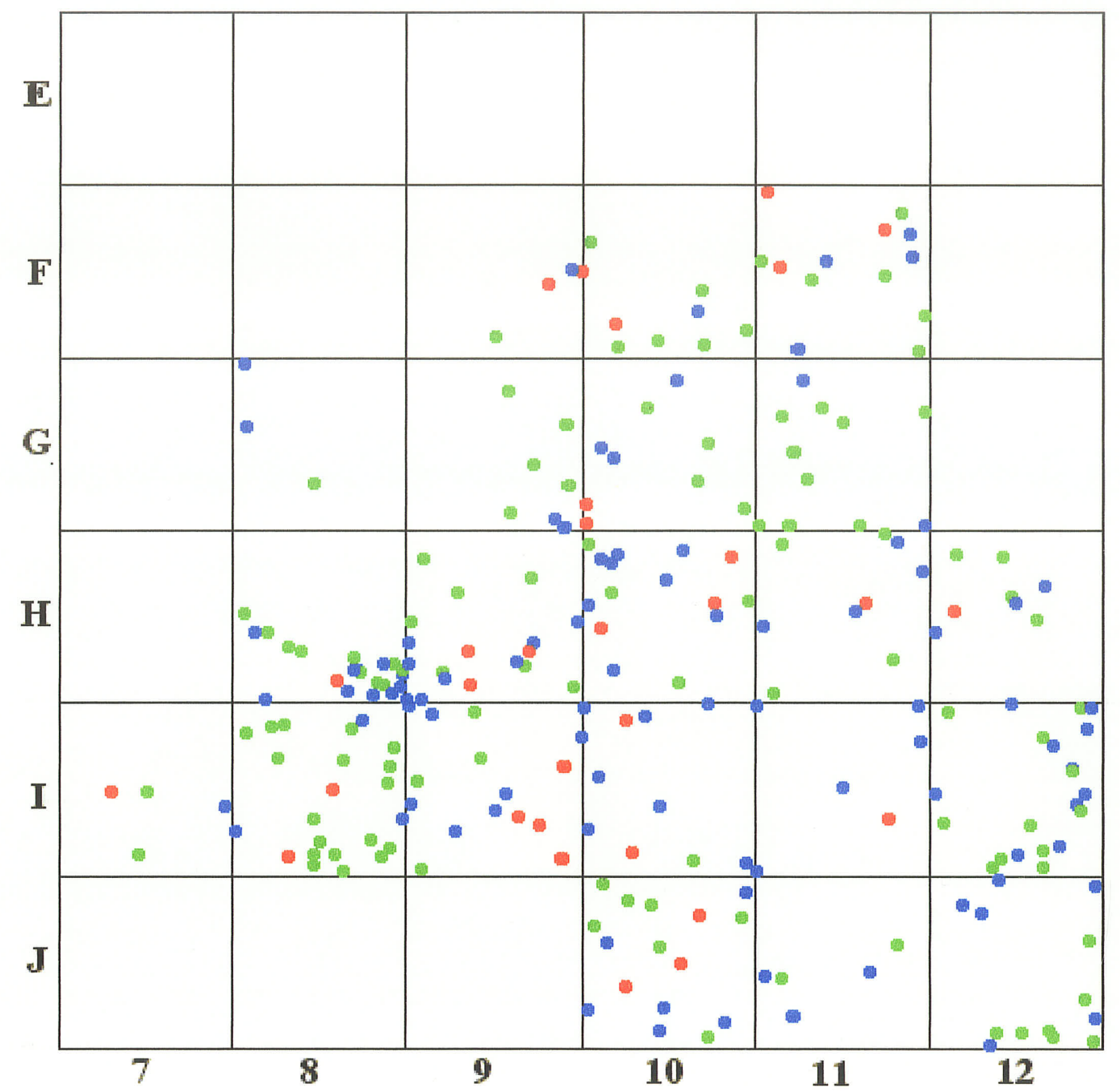

\section{Legend}

Bone
Flalses and Chips
Tools

Figure 9: Total Artefact Distribution, Re-Sampled Dataset - Level IV 


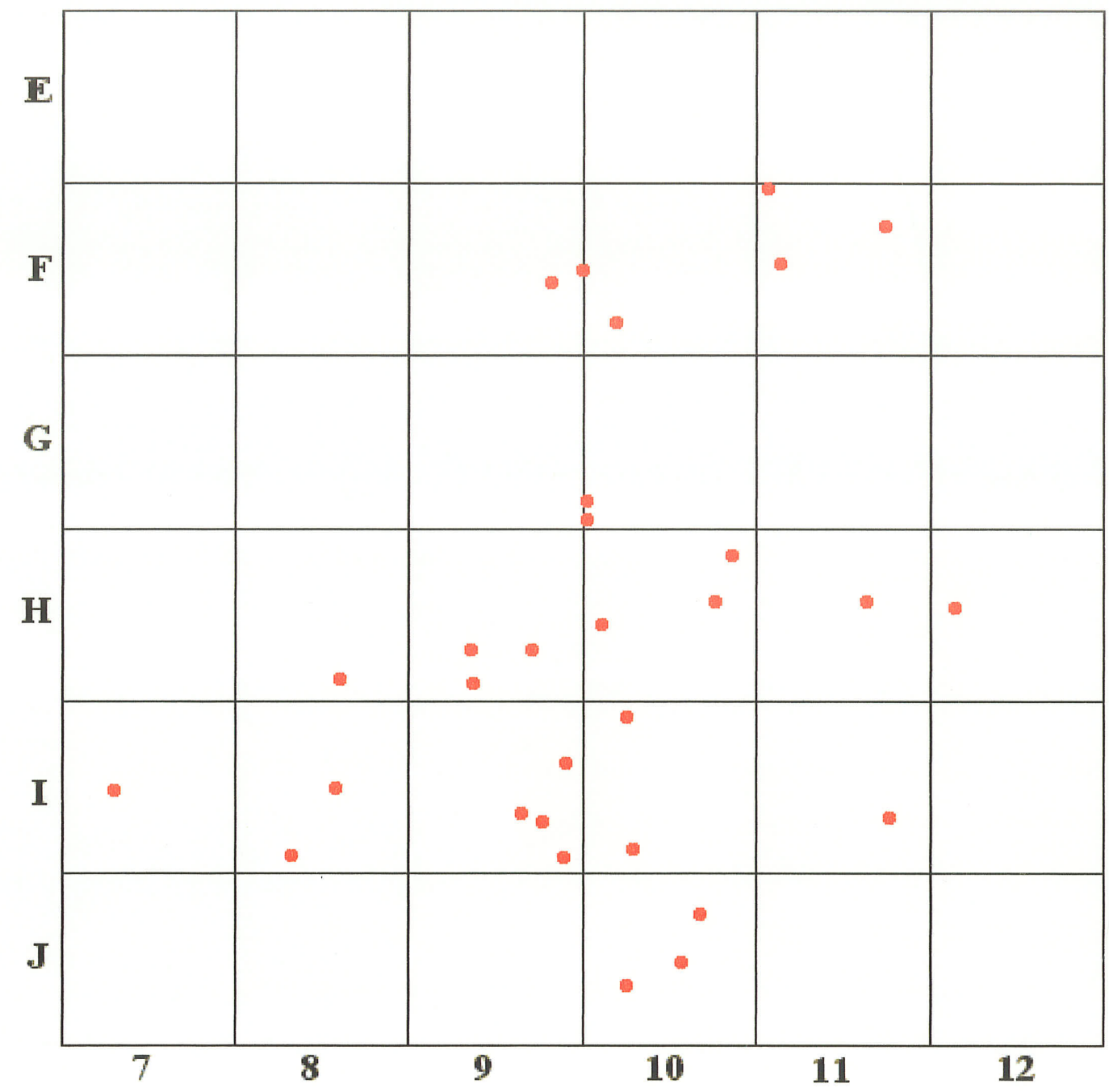

Figure 10: Stone Tool Distribution, Re-Sampled Dataset - Level IV 


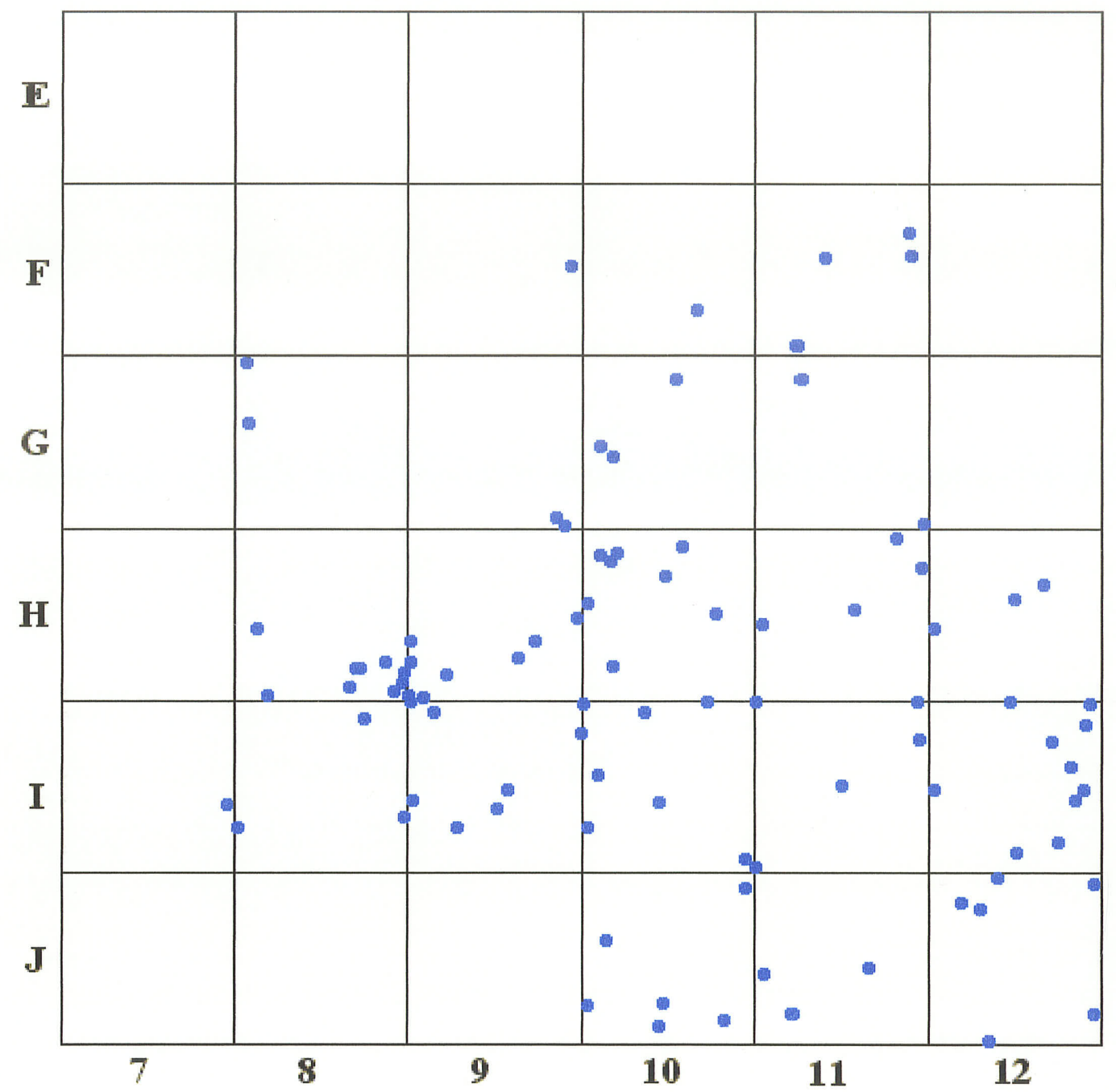

Figure 11: Stone Flakes and Chips Distribution, Re-Sampled Dataset - Level IV 


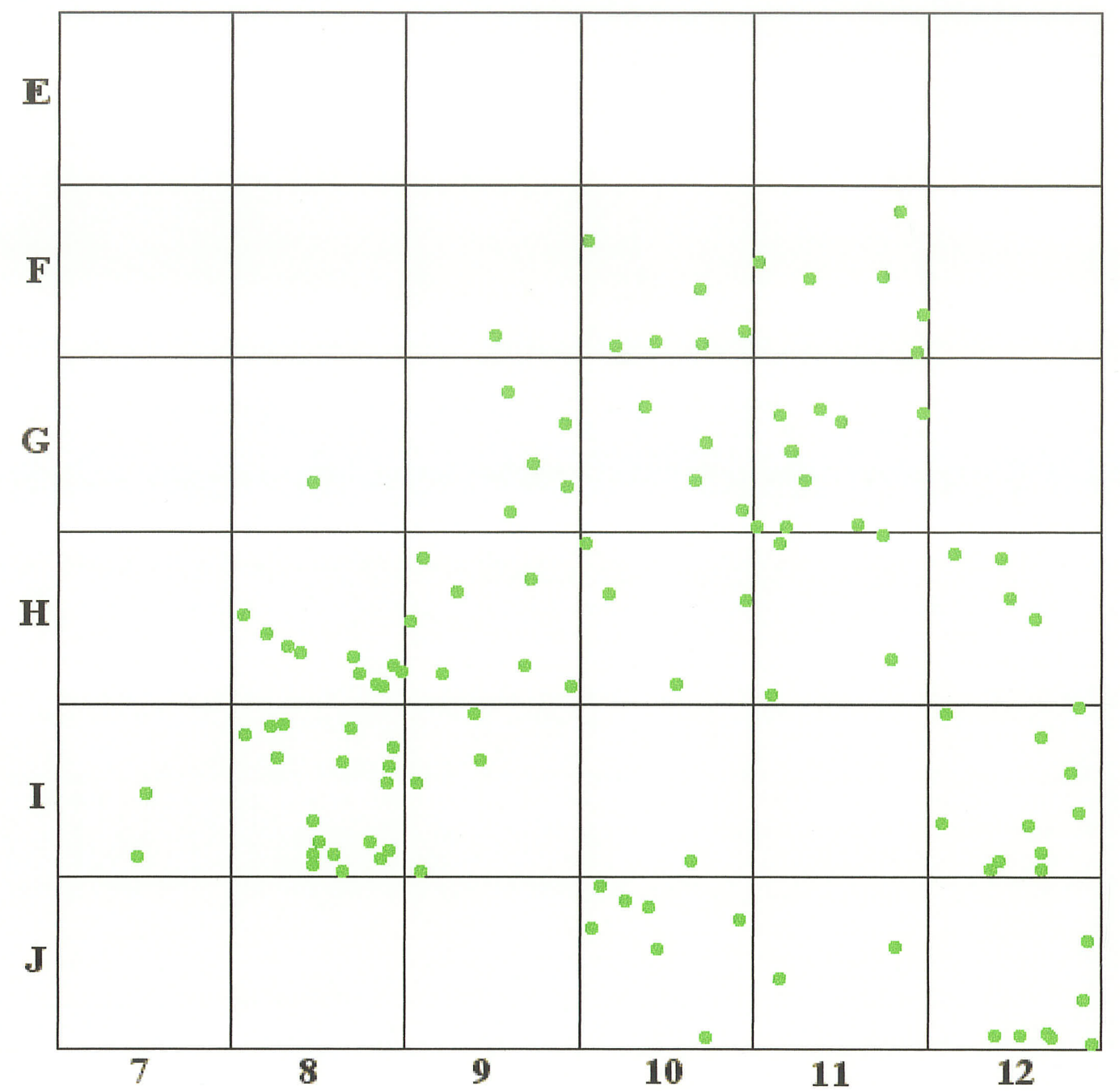

Figure 12: Bone Distribution, Re-Sampled Dataset - Level IV 


\section{- Appendix C - K-Means Cluster Solutions \\ Kintigh's Tools for Quantitative Archaeology}




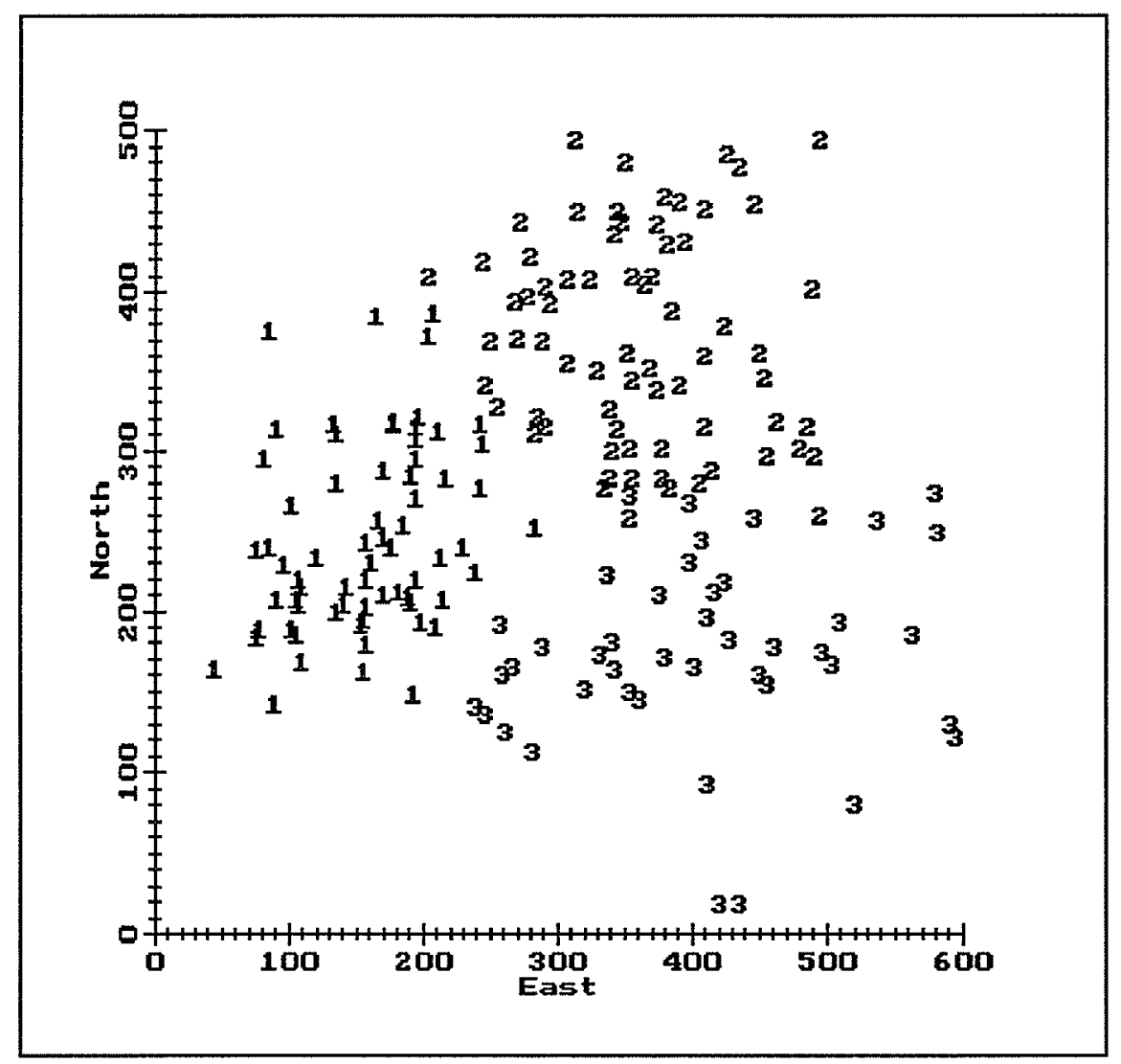

Figure 1: 3 Cluster Solution - Level II/2, with Artefacts Identified by Cluster Number 


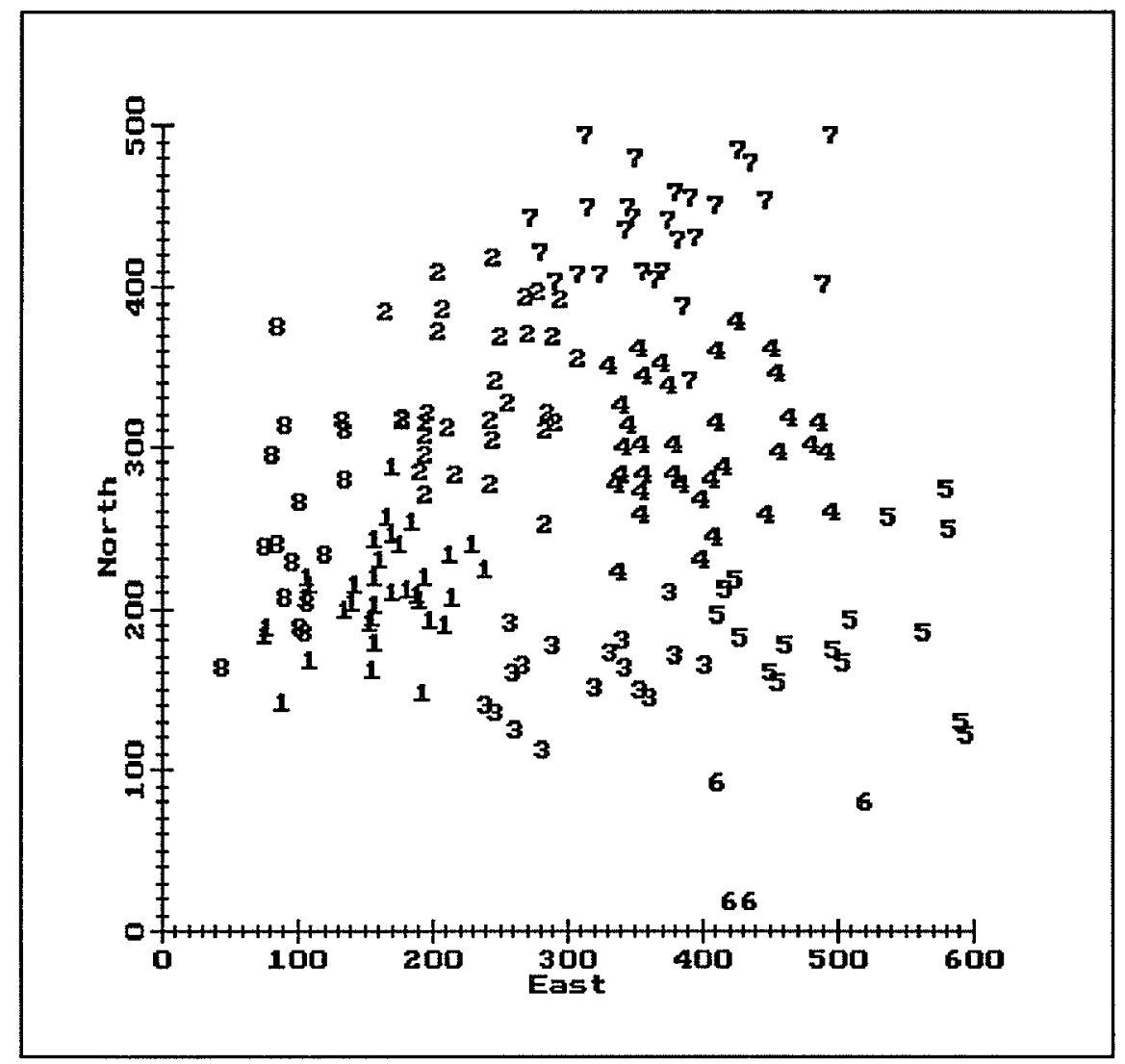

Figure 2: 8 Cluster Solution - Level II/2, with Artefacts Identified by Cluster Number 


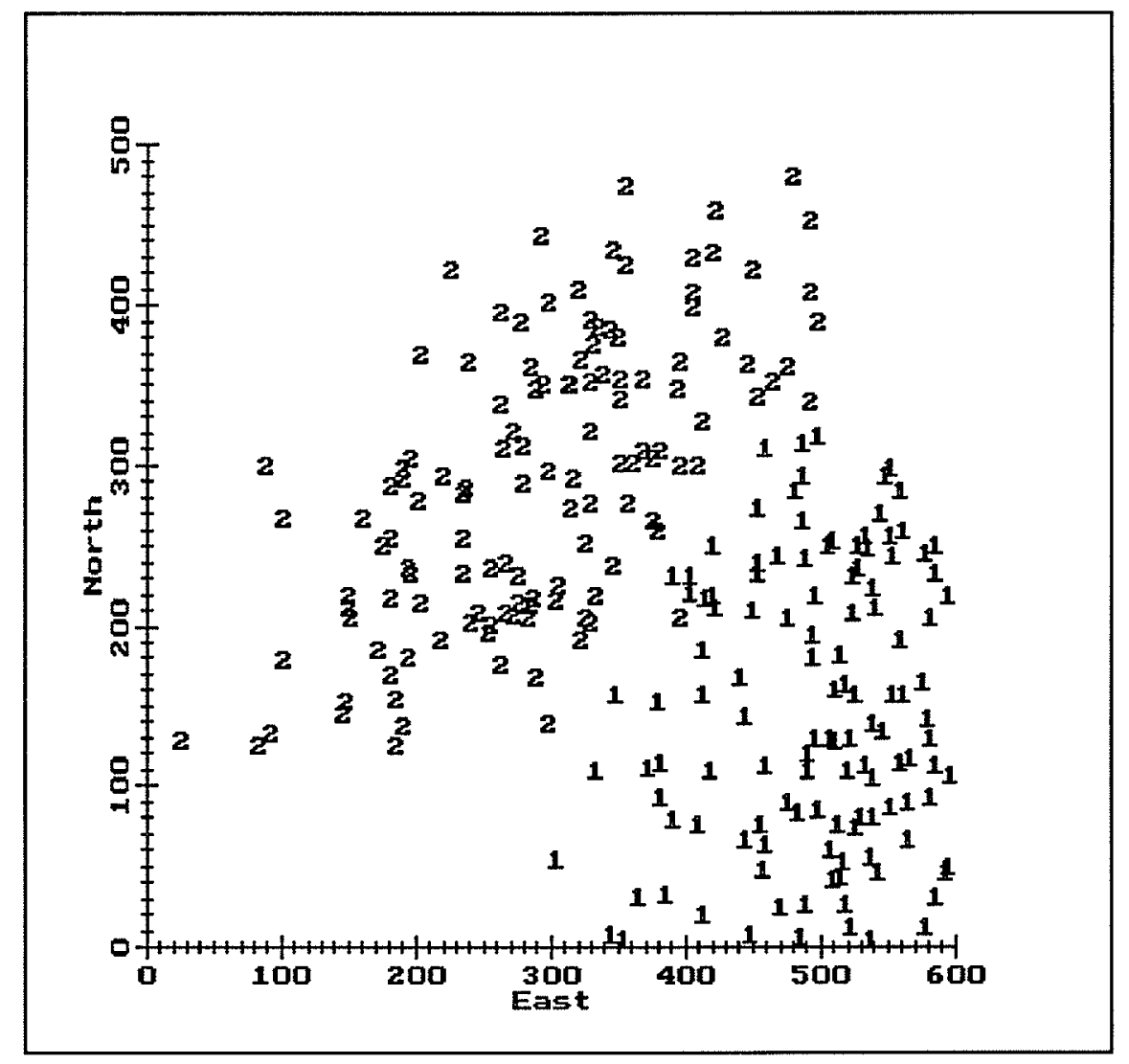

Figure 3: 2 Cluster Solution - Level III, with Artefacts Identified by Cluster Number 


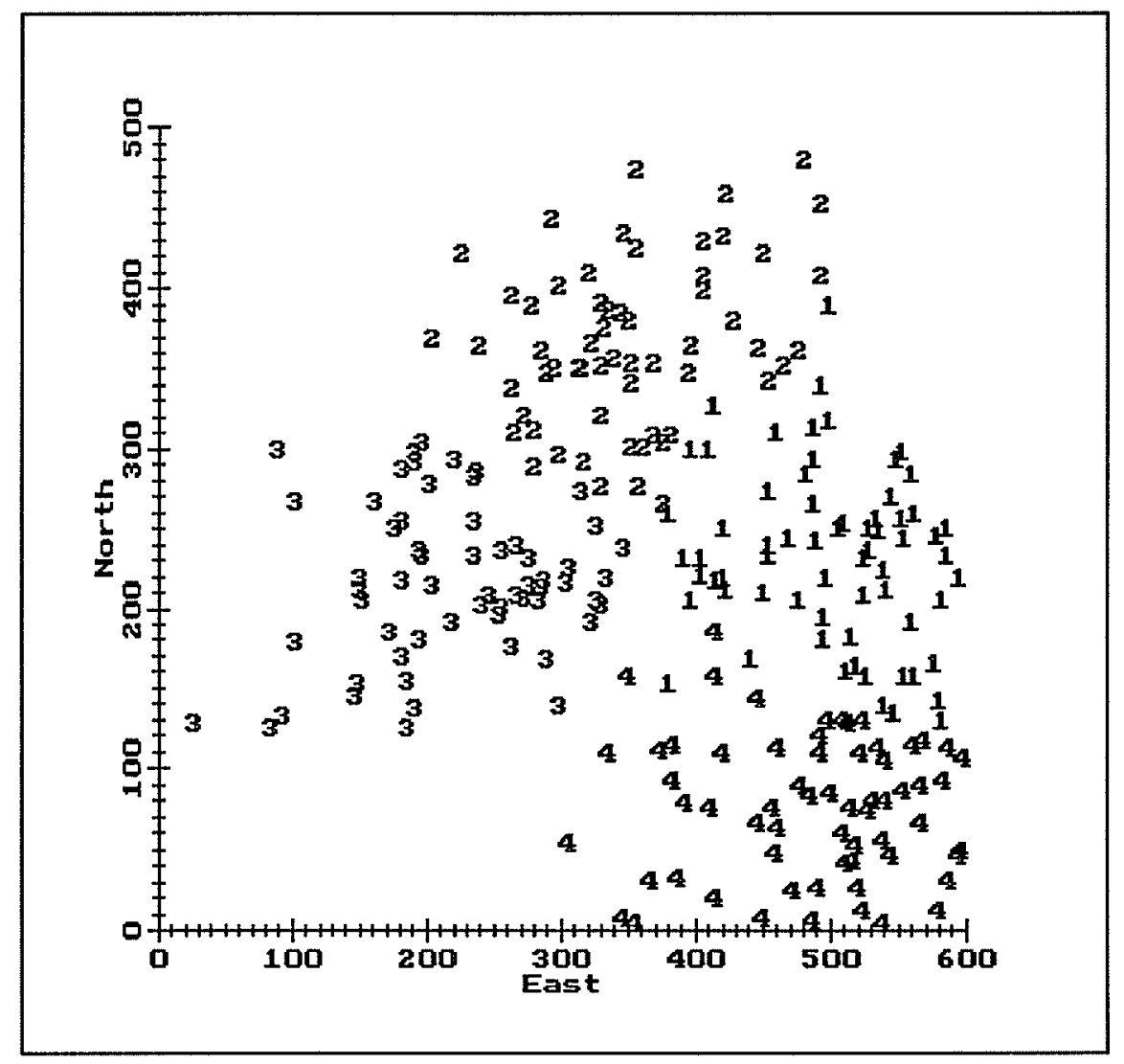

Figure 4: 4 Cluster Solution - Level III, with Artefacts Identified by Cluster Number 


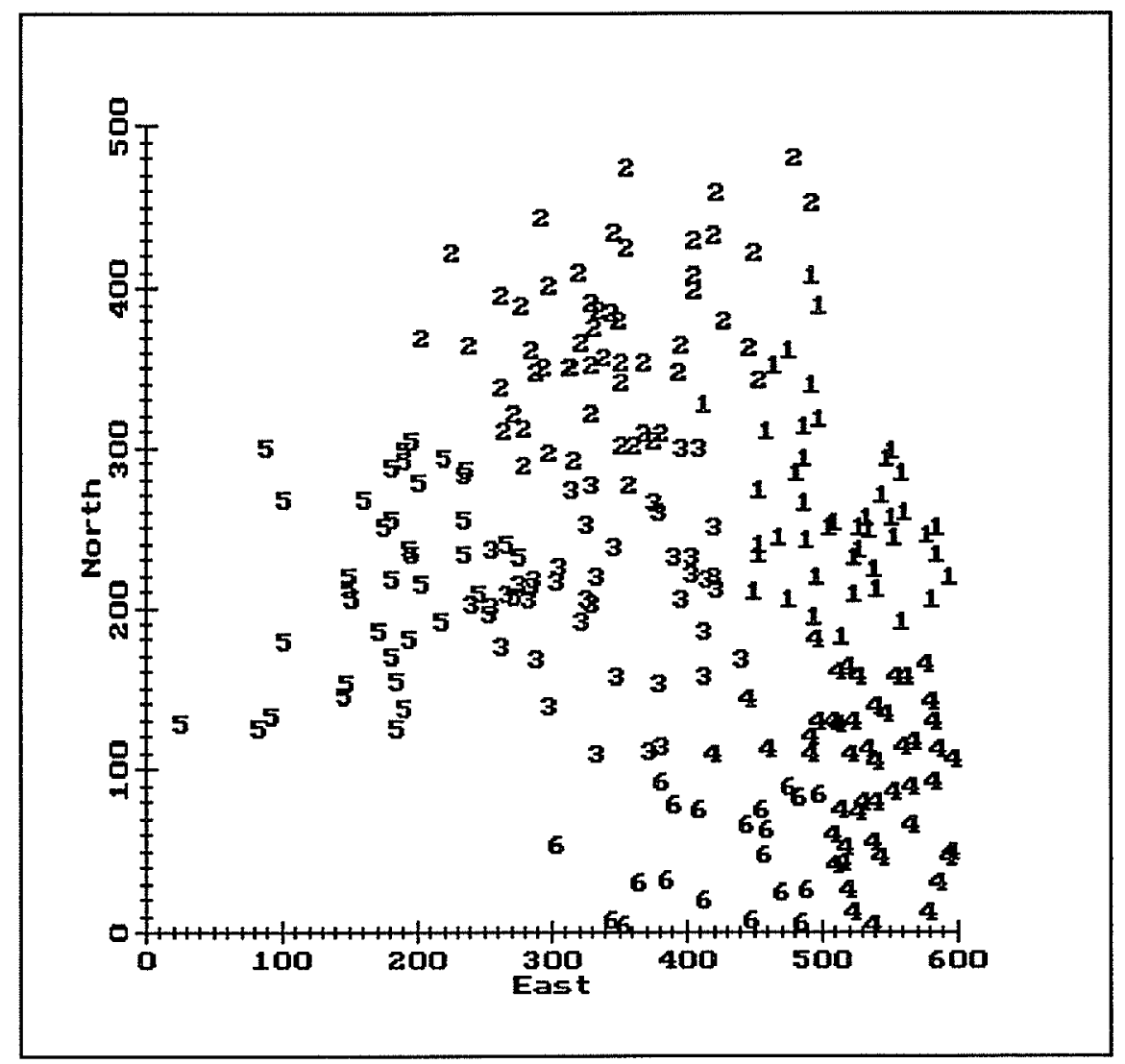

Figure 5: 6 Cluster Solution - Level III, with Artefacts Identified by Cluster Number 


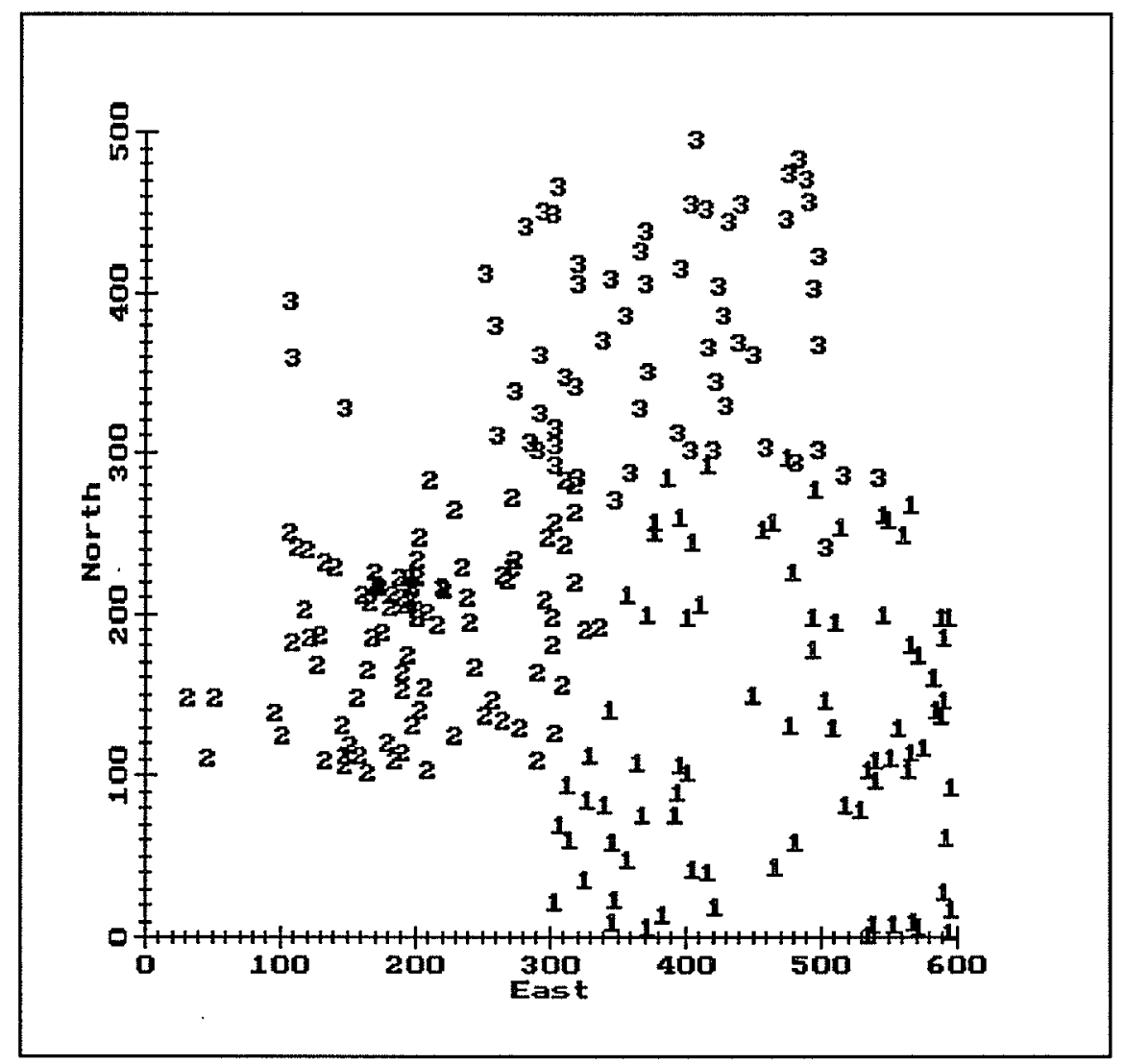

Figure 6: 3 Cluster Solution - Level IV, with Artefacts Identified by Cluster Number 


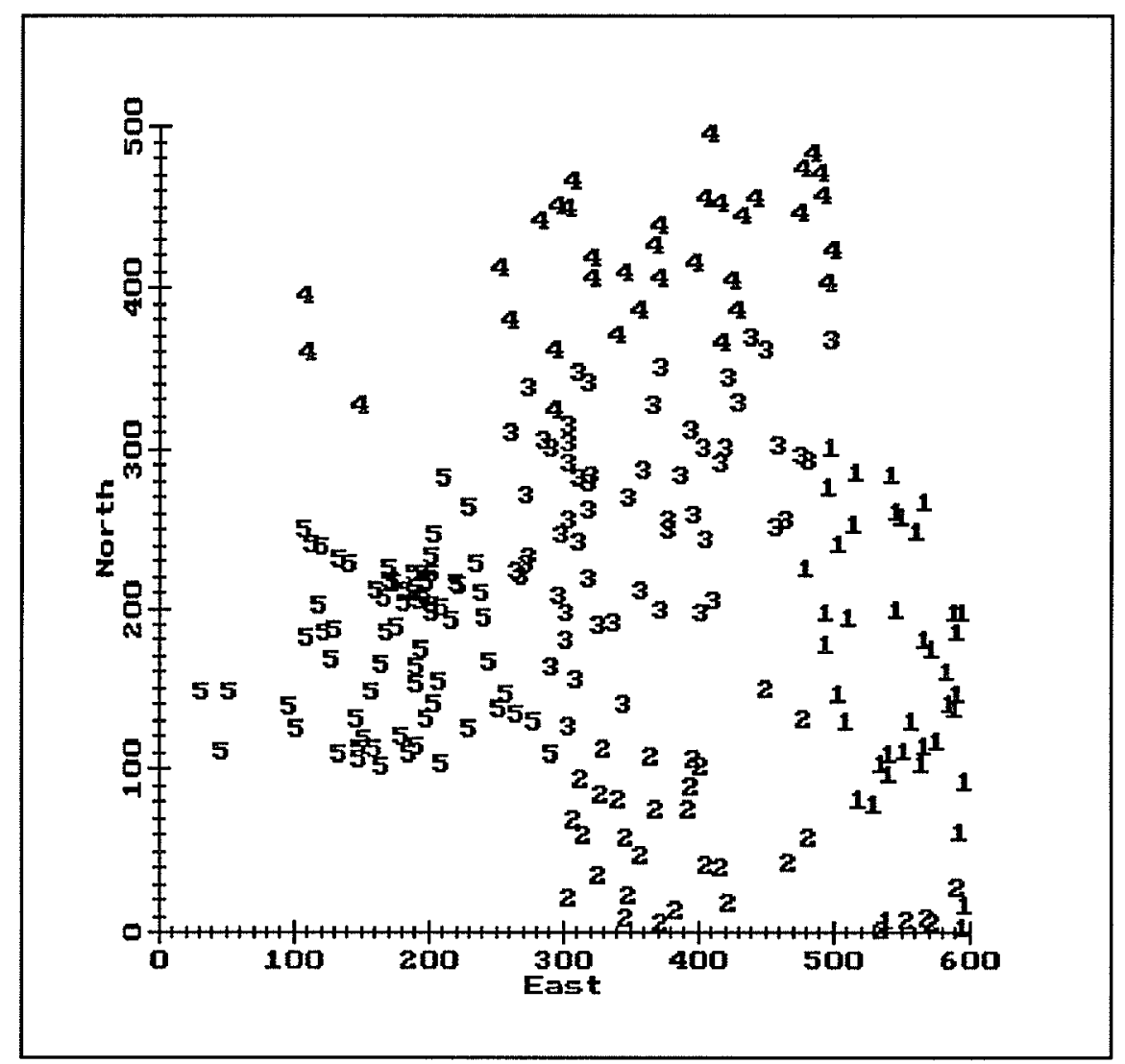

Figure 7: 5 Cluster Solution - Level IV, with Artefacts Identified by Cluster Number 
- Appendix D -

Chi-Square Significance Results 


\section{Chi-Square Contingency Table Results - Re-sampled Dataset Trials}

\section{Level II/2}

\begin{tabular}{|c|c|c|c|c|c|c|c|c|}
\hline $\begin{array}{c}\text { Level } \\
\text { II/2 }\end{array}$ & \multicolumn{3}{|c|}{ Observed Frequency } & Total & \multicolumn{3}{c|}{ Expected Frequency } & Total \\
\hline & Bone & $\begin{array}{c}\text { Chips and } \\
\text { Flakes }\end{array}$ & $\begin{array}{c}\text { Stone } \\
\text { Tools }\end{array}$ & & Bone & $\begin{array}{c}\text { Chips and } \\
\text { Flakes }\end{array}$ & $\begin{array}{c}\text { Stone } \\
\text { Tools }\end{array}$ & \\
\hline Trial 1 & 104 & 49 & 28 & $\mathbf{1 8 1}$ & 105.5 & 48.0 & 27.5 & $\mathbf{1 8 1}$ \\
\hline Trial 2 & 107 & 47 & 27 & $\mathbf{1 8 1}$ & 105.5 & 48.0 & 27.5 & $\mathbf{1 8 1}$ \\
\hline Total & $\mathbf{2 1 1}$ & $\mathbf{9 6}$ & $\mathbf{5 5}$ & $\mathbf{3 6 2}$ & $\mathbf{2 1 1}$ & $\mathbf{9 6}$ & $\mathbf{5 5}$ & $\mathbf{3 6 2}$ \\
\hline
\end{tabular}

Chi-Square Value: 0.10 Degrees of Freedom: 2

Critical Value: 5.99

Table 1: Chi-Square Significance Results Level II/2 Trials 1 and 2

\begin{tabular}{|c|c|c|c|c|c|c|c|c|}
\hline $\begin{array}{c}\text { Level } \\
\text { II/2 }\end{array}$ & \multicolumn{3}{|c|}{ Observed Frequency } & Total & \multicolumn{3}{c|}{ Expected Frequency } & Total \\
\hline & Bone & $\begin{array}{c}\text { Chips and } \\
\text { Flakes }\end{array}$ & $\begin{array}{c}\text { Stone } \\
\text { Tools }\end{array}$ & & Bone & $\begin{array}{c}\text { Chips and } \\
\text { Flakes }\end{array}$ & $\begin{array}{c}\text { Stone } \\
\text { Tools }\end{array}$ & \\
\hline Trial 1 & 104 & 49 & 28 & $\mathbf{1 8 1}$ & 105.5 & 48.5 & 27.0 & $\mathbf{1 8 1}$ \\
\hline Trial 3 & 107 & 48 & 26 & $\mathbf{1 8 1}$ & 105.5 & 48.5 & 27.0 & $\mathbf{1 8 1}$ \\
\hline Total & $\mathbf{2 1 1}$ & $\mathbf{9 7}$ & $\mathbf{5 4}$ & $\mathbf{3 6 2}$ & $\mathbf{2 1 1}$ & $\mathbf{9 7}$ & $\mathbf{5 4}$ & $\mathbf{3 6 2}$ \\
\hline
\end{tabular}

Chi-Square Value: 0.13 Degrees of Freedom: 2

Critical Value: 5.99

Table 2: Chi-Square Significance Results Level II/2 Trials 1 and 3

\begin{tabular}{|c|c|c|c|c|c|c|c|c|}
\hline $\begin{array}{c}\text { Level } \\
\text { II/2 }\end{array}$ & \multicolumn{3}{|c|}{ Observed Frequency } & Total & \multicolumn{3}{c|}{ Expected Frequency } & Total \\
\hline & Bone & $\begin{array}{c}\text { Chips and } \\
\text { Flakes }\end{array}$ & $\begin{array}{c}\text { Stone } \\
\text { Tools }\end{array}$ & & Bone & $\begin{array}{c}\text { Chips and } \\
\text { Flakes }\end{array}$ & $\begin{array}{c}\text { Stone } \\
\text { Tools }\end{array}$ & \\
\hline Trial 2 & 107 & 47 & 27 & $\mathbf{1 8 1}$ & 107.0 & 47.5 & 26.5 & $\mathbf{1 8 1}$ \\
\hline Trial 3 & 107 & 48 & 26 & $\mathbf{1 8 1}$ & 107.0 & 47.5 & 26.5 & $\mathbf{1 8 1}$ \\
\hline Total & $\mathbf{2 1 4}$ & $\mathbf{9 5}$ & $\mathbf{5 3}$ & $\mathbf{3 6 2}$ & $\mathbf{2 1 1}$ & $\mathbf{9 6}$ & $\mathbf{5 5}$ & $\mathbf{3 6 2}$ \\
\hline
\end{tabular}

Chi-Square Value: 0.03 Degrees of Freedom: 2

Critical Value: 5.99

Table 3: Chi-Square Significance Results Level II/2 Trials 2 and 3 


\section{Chi-Square Contingency Table Results - Re-sampled Dataset Trials}

\section{Level III}

\begin{tabular}{|c|c|c|c|c|c|c|c|c|}
\hline $\begin{array}{c}\text { Level } \\
\text { III }\end{array}$ & \multicolumn{3}{|c|}{ Observed Frequency } & Total & \multicolumn{3}{|c|}{ Expected Frequency } & Total \\
\hline & Bone & $\begin{array}{c}\text { Chips and } \\
\text { Flakes }\end{array}$ & $\begin{array}{c}\text { Stone } \\
\text { Tools }\end{array}$ & & Bone & $\begin{array}{c}\text { Chips and } \\
\text { Flakes }\end{array}$ & $\begin{array}{c}\text { Stone } \\
\text { Tools }\end{array}$ & \\
\hline Trial 1 & 113 & 88 & 47 & $\mathbf{2 4 8}$ & 113.5 & 88.0 & 46.5 & $\mathbf{2 4 8}$ \\
\hline Trial 2 & 114 & $\mathbf{8 8}$ & 46 & $\mathbf{2 4 8}$ & 113.5 & 88.0 & 46.5 & $\mathbf{2 4 8}$ \\
\hline Total & $\mathbf{2 2 7}$ & $\mathbf{1 7 6}$ & $\mathbf{9 3}$ & $\mathbf{4 9 6}$ & $\mathbf{2 2 7}$ & $\mathbf{1 7 6}$ & $\mathbf{9 3}$ & $\mathbf{4 9 6}$ \\
\hline
\end{tabular}

Chi-Square Value: 0.02 Degrees of Freedom: 2

Critical Value: 5.99

Table 4: Chi-Square Significance Results Level III Trials 1 and 2

\begin{tabular}{|c|c|c|c|c|c|c|c|c|}
\hline $\begin{array}{c}\text { Level } \\
\text { III }\end{array}$ & \multicolumn{3}{|c|}{ Observed Frequency } & Total & \multicolumn{3}{|c|}{ Expected Frequency } & Total \\
\hline & Bone & $\begin{array}{c}\text { Chips and } \\
\text { Flakes }\end{array}$ & $\begin{array}{c}\text { Stone } \\
\text { Tools }\end{array}$ & & Bone & $\begin{array}{c}\text { Chips and } \\
\text { Flakes }\end{array}$ & $\begin{array}{c}\text { Stone } \\
\text { Tools }\end{array}$ & \\
\hline Trial 1 & 113 & 88 & 47 & $\mathbf{2 4 8}$ & 114.5 & 88.0 & 45.5 & $\mathbf{2 4 8}$ \\
\hline Trial 3 & 116 & 88 & 44 & $\mathbf{2 4 8}$ & 114.5 & 88.0 & 45.5 & $\mathbf{2 4 8}$ \\
\hline Total & $\mathbf{2 2 9}$ & $\mathbf{1 7 6}$ & $\mathbf{9 1}$ & $\mathbf{4 9 6}$ & $\mathbf{2 2 9}$ & $\mathbf{1 7 6}$ & $\mathbf{9 1}$ & $\mathbf{4 9 6}$ \\
\hline
\end{tabular}

Chi-Square Value: 0.14 Degrees of Freedom: 2

Critical Value: 5.99

Table 5: Chi-Square Significance Results Level III Trials 1 and 3

\begin{tabular}{|c|c|c|c|c|c|c|c|c|}
\hline $\begin{array}{c}\text { Level } \\
\text { III }\end{array}$ & \multicolumn{3}{|c|}{ Observed Frequency } & Total & \multicolumn{3}{c|}{ Expected Frequency } & Total \\
\hline & Bone & $\begin{array}{c}\text { Chips and } \\
\text { Flakes }\end{array}$ & $\begin{array}{c}\text { Stone } \\
\text { Tools }\end{array}$ & & Bone & $\begin{array}{c}\text { Chips and } \\
\text { Flakes }\end{array}$ & $\begin{array}{c}\text { Stone } \\
\text { Tools }\end{array}$ & \\
\hline Trial 2 & 114 & 88 & 46 & $\mathbf{2 4 8}$ & 115.0 & 88.0 & 45.0 & $\mathbf{2 4 8}$ \\
\hline Trial 3 & 116 & 88 & 44 & $\mathbf{2 4 8}$ & 115.0 & 88.0 & 45.0 & $\mathbf{2 4 8}$ \\
\hline Total & $\mathbf{2 3 0}$ & $\mathbf{1 7 6}$ & $\mathbf{9 0}$ & $\mathbf{4 9 6}$ & $\mathbf{2 3 0}$ & $\mathbf{1 7 6}$ & $\mathbf{9 0}$ & $\mathbf{4 9 6}$ \\
\hline
\end{tabular}

Chi-Square Value: 0.06 Degrees of Freedom: 2

Critical Value: 5.99

Table 6: Chi-Square Significance Results Level III Trials 2 and 3 
Chi-Square Contingency Table Results - Re-sampled Dataset Trials

\section{Level IV}

\begin{tabular}{|c|c|c|c|c|c|c|c|c|}
\hline $\begin{array}{c}\text { Level } \\
\text { IV }\end{array}$ & \multicolumn{3}{|c|}{ Observed Frequency } & Total & \multicolumn{3}{c|}{ Expected Frequency } & Total \\
\hline & Bone & $\begin{array}{c}\text { Chips and } \\
\text { Flakes }\end{array}$ & $\begin{array}{c}\text { Stone } \\
\text { Tools }\end{array}$ & & Bone & $\begin{array}{c}\text { Chips and } \\
\text { Flakes }\end{array}$ & $\begin{array}{c}\text { Stone } \\
\text { Tools }\end{array}$ & \\
\hline Trial 1 & 115 & 95 & 29 & $\mathbf{2 3 9}$ & 118.5 & 92.0 & 28.5 & $\mathbf{2 3 9}$ \\
\hline Trial 2 & 122 & 89 & 28 & $\mathbf{2 3 9}$ & 118.5 & 92.0 & 28.5 & $\mathbf{2 3 9}$ \\
\hline Total & $\mathbf{2 3 7}$ & $\mathbf{1 8 4}$ & $\mathbf{5 7}$ & $\mathbf{4 7 8}$ & $\mathbf{2 3 7}$ & $\mathbf{1 8 4}$ & $\mathbf{5 7}$ & $\mathbf{4 7 8}$ \\
\hline
\end{tabular}

Chi-Square Value: 0.42 Degrees of Freedom: 2

Critical Value: 5.99

Table 7: Chi-Square Significance Results Level IV Trials 1 and 2

\begin{tabular}{|c|c|c|c|c|c|c|c|c|}
\hline $\begin{array}{c}\text { Level } \\
\text { IV }\end{array}$ & \multicolumn{3}{|c|}{ Observed Frequency } & Total & \multicolumn{3}{c|}{ Expected Frequency } & Total \\
\hline & Bone & $\begin{array}{c}\text { Chips and } \\
\text { Flakes }\end{array}$ & $\begin{array}{c}\text { Stone } \\
\text { Tools }\end{array}$ & & Bone & $\begin{array}{c}\text { Chips and } \\
\text { Flakes }\end{array}$ & $\begin{array}{c}\text { Stone } \\
\text { Tools }\end{array}$ & \\
\hline Trial 1 & 115 & 95 & 29 & $\mathbf{2 3 9}$ & 114.0 & 95.5 & 29.5 & $\mathbf{2 3 9}$ \\
\hline Trial 3 & 113 & 96 & 30 & $\mathbf{2 3 9}$ & 114.0 & 95.5 & 29.5 & $\mathbf{2 3 9}$ \\
\hline Total & $\mathbf{2 2 8}$ & $\mathbf{1 9 1}$ & $\mathbf{5 9}$ & $\mathbf{4 7 8}$ & $\mathbf{2 2 8}$ & $\mathbf{1 9 1}$ & $\mathbf{5 9}$ & $\mathbf{4 7 8}$ \\
\hline
\end{tabular}

Chi-Square Value: 0.04 Degrees of Freedom: 2

Critical Value: 5.99

Table 8: Chi-Square Significance Results Level IV Trials 1 and 3

\begin{tabular}{|c|c|c|c|c|c|c|c|c|}
\hline $\begin{array}{c}\text { Level } \\
\text { IV }\end{array}$ & \multicolumn{3}{|c|}{ Observed Frequency } & Total & \multicolumn{3}{c|}{ Expected Frequency } & Total \\
\hline & Bone & $\begin{array}{c}\text { Chips and } \\
\text { Flakes }\end{array}$ & $\begin{array}{c}\text { Stone } \\
\text { Tools }\end{array}$ & & Bone & $\begin{array}{c}\text { Chips and } \\
\text { Flakes }\end{array}$ & $\begin{array}{c}\text { Stone } \\
\text { Tools }\end{array}$ & \\
\hline Trial 2 & 122 & 89 & 28 & $\mathbf{2 3 9}$ & 117.5 & 92.5 & 29.0 & $\mathbf{2 3 9}$ \\
\hline Trial 3 & 113 & 96 & 30 & $\mathbf{2 3 9}$ & 117.5 & 92.5 & 29.0 & $\mathbf{2 3 9}$ \\
\hline Total & $\mathbf{2 3 5}$ & $\mathbf{1 8 5}$ & $\mathbf{5 8}$ & $\mathbf{4 7 8}$ & $\mathbf{2 3 5}$ & $\mathbf{1 8 5}$ & $\mathbf{5 8}$ & $\mathbf{4 7 8}$ \\
\hline
\end{tabular}

Chi-Square Value: 0.68 Degrees of Freedom: 2

Critical Value: 5.99

Table 9: Chi-Square Significance Results Level IV Trials 2 and 3 
Chi-Square Contingency Table Results - 3 Cluster Solution Level II/2

\begin{tabular}{|c|c|c|c|c|c|c|c|c|}
\hline Level II/2 & \multicolumn{3}{|c|}{ Observed Frequency } & Total & \multicolumn{3}{|c|}{ Expected Frequency } & Total \\
\hline & Bone & $\begin{array}{c}\text { Chips and } \\
\text { Flakes }\end{array}$ & $\begin{array}{c}\text { Stone } \\
\text { Tools }\end{array}$ & & Bone & $\begin{array}{c}\text { Chips and } \\
\text { Flakes }\end{array}$ & $\begin{array}{c}\text { Stone } \\
\text { Tools }\end{array}$ & \\
\hline Cluster 3-1 & 39 & 23 & 6 & $\mathbf{6 8}$ & 41.4 & 18.7 & 7.9 & $\mathbf{6 8}$ \\
\hline Cluster 3-2 & 45 & 15 & 10 & $\mathbf{7 0}$ & 42.6 & 19.3 & 8.1 & $\mathbf{7 0}$ \\
\hline Total & $\mathbf{8 4}$ & $\mathbf{3 8}$ & $\mathbf{1 6}$ & $\mathbf{1 3 8}$ & $\mathbf{8 4}$ & $\mathbf{3 8}$ & $\mathbf{1 6}$ & $\mathbf{1 3 8}$ \\
\hline
\end{tabular}

Chi-Square Value: 3.08 Degrees of Freedom: 2

Critical Value: 5.99

Table 10: Chi-Square Results 3 Cluster Solution Level II/2, Clusters 3-1 and 3-2

\begin{tabular}{|c|c|c|c|c|c|c|c|c|}
\hline Level II/2 & \multicolumn{3}{|c|}{ Observed Frequency } & Total & \multicolumn{3}{c|}{ Expected Frequency } & Total \\
\hline & Bone & $\begin{array}{c}\text { Chips and } \\
\text { Flakes }\end{array}$ & $\begin{array}{c}\text { Stone } \\
\text { Tools }\end{array}$ & & Bone & $\begin{array}{c}\text { Chips and } \\
\text { Flakes }\end{array}$ & $\begin{array}{c}\text { Stone } \\
\text { Tools }\end{array}$ & \\
\hline Cluster 3-1 & 39 & 23 & 6 & $\mathbf{6 8}$ & 38.0 & 20.2 & 9.8 & $\mathbf{6 8}$ \\
\hline Cluster 3-3 & 23 & 10 & 10 & $\mathbf{4 3}$ & 24.0 & 12.8 & 6.2 & $\mathbf{4 3}$ \\
\hline Total & $\mathbf{6 2}$ & $\mathbf{3 3}$ & $\mathbf{1 6}$ & $\mathbf{1 1 1}$ & $\mathbf{6 2}$ & $\mathbf{3 3}$ & $\mathbf{1 6}$ & $\mathbf{1 1 1}$ \\
\hline
\end{tabular}

Chi-Square Value: 4.87 Degrees of Freedom: 2

Critical Value: 5.99

Table 11: Chi-Square Results 3 Cluster Solution Level II/2, Clusters 3-1 and 3-3

\begin{tabular}{|c|c|c|c|c|c|c|c|c|}
\hline Level II/2 & \multicolumn{3}{|c|}{ Observed Frequency } & Total & \multicolumn{3}{c|}{ Expected Frequency } & Total \\
\hline & Bone & $\begin{array}{c}\text { Chips and } \\
\text { Flakes }\end{array}$ & $\begin{array}{c}\text { Stone } \\
\text { Tools }\end{array}$ & & Bone & $\begin{array}{c}\text { Chips and } \\
\text { Flakes }\end{array}$ & $\begin{array}{c}\text { Stone } \\
\text { Tools }\end{array}$ & \\
\hline Cluster 3-2 & 45 & 15 & 10 & $\mathbf{7 0}$ & 42.1 & 15.5 & 12.4 & $\mathbf{7 0}$ \\
\hline Cluster 3-3 & 23 & 10 & 10 & $\mathbf{4 3}$ & 25.9 & 9.5 & 7.6 & $\mathbf{4 3}$ \\
\hline Total & $\mathbf{6 8}$ & $\mathbf{2 5}$ & $\mathbf{2 0}$ & $\mathbf{1 1 3}$ & $\mathbf{6 8}$ & $\mathbf{2 5}$ & $\mathbf{2 0}$ & $\mathbf{1 1 3}$ \\
\hline
\end{tabular}

Chi-Square Value: 1.77 Degrees of Freedom: 2

Critical Value: 5.99

Table 12: Chi-Square Results 3 Cluster Solution Level II/2, Clusters 3-2 and 3-3 


\section{Chi-Square Contingency Table Results -8 Cluster Solution Level II/2}

\begin{tabular}{|c|c|c|c|c|c|c|c|c|}
\hline Level II/2 & \multicolumn{3}{|c|}{ Observed Frequency } & Total & \multicolumn{3}{|c|}{ Expected Frequency } & Total \\
\hline & Bone & $\begin{array}{c}\text { Chips and } \\
\text { Flakes }\end{array}$ & $\begin{array}{c}\text { Stone } \\
\text { Tools }\end{array}$ & & Bone & $\begin{array}{c}\text { Chips and } \\
\text { Flakes }\end{array}$ & $\begin{array}{c}\text { Stone } \\
\text { Tools }\end{array}$ & \\
\hline Cluster 8-1 & 16 & 16 & 3 & $\mathbf{3 5}$ & 20.7 & 11.7 & 2.7 & $\mathbf{3 5}$ \\
\hline Cluster 8-2 & 23 & 6 & 2 & $\mathbf{3 1}$ & 18.3 & 10.3 & 2.3 & $\mathbf{3 1}$ \\
\hline Total & $\mathbf{3 9}$ & $\mathbf{2 2}$ & $\mathbf{5}$ & $\mathbf{6 6}$ & $\mathbf{3 9}$ & $\mathbf{2 2}$ & $\mathbf{5}$ & $\mathbf{6 6}$ \\
\hline
\end{tabular}

Chi-Square Value: 5.78 Degrees of Freedom: 2

Critical Value: 5.99

Table 13: Chi-Square Results 8 Cluster Solution Level II/2, Clusters 8-1 and 8-2

\begin{tabular}{|c|c|c|c|c|c|c|c|c|}
\hline Level II/2 & \multicolumn{3}{|c|}{ Observed Frequency } & Total & \multicolumn{3}{c|}{ Expected Frequency } & Total \\
\hline & Bone & $\begin{array}{c}\text { Chips and } \\
\text { Flakes }\end{array}$ & $\begin{array}{c}\text { Stone } \\
\text { Tools }\end{array}$ & & Bone & $\begin{array}{c}\text { Chips and } \\
\text { Flakes }\end{array}$ & $\begin{array}{c}\text { Stone } \\
\text { Tools }\end{array}$ & \\
\hline Cluster 8-1 & 16 & 16 & 3 & $\mathbf{3 5}$ & 14.8 & 14.1 & 6.1 & $\mathbf{3 5}$ \\
\hline Cluster 8-3 & 6 & 5 & 6 & $\mathbf{1 7}$ & 7.2 & 6.9 & 2.9 & $\mathbf{1 7}$ \\
\hline Total & $\mathbf{2 2}$ & $\mathbf{2 1}$ & $\mathbf{9}$ & $\mathbf{5 2}$ & $\mathbf{2 2}$ & $\mathbf{2 1}$ & $\mathbf{9}$ & $\mathbf{5 2}$ \\
\hline
\end{tabular}

Chi-Square Value: 5.77 Degrees of Freedom: 2

Critical Value: 5.99

Table 14: Chi-Square Results 8 Cluster Solution Level II/2, Clusters 8-1 and 8-3

\begin{tabular}{|c|c|c|c|c|c|c|c|c|}
\hline Level II/2 & \multicolumn{3}{|c|}{ Observed Frequency } & Total & \multicolumn{3}{c|}{ Expected Frequency } & Total \\
\hline & Bone & $\begin{array}{c}\text { Chips and } \\
\text { Flakes }\end{array}$ & $\begin{array}{c}\text { Stone } \\
\text { Tools }\end{array}$ & & Bone & $\begin{array}{c}\text { Chips and } \\
\text { Flakes }\end{array}$ & $\begin{array}{c}\text { Stone } \\
\text { Tools }\end{array}$ & \\
\hline Cluster 8-1 & 16 & 16 & 3 & $\mathbf{3 5}$ & 19.0 & 10.5 & 5.5 & $\mathbf{3 5}$ \\
\hline Cluster 8-4 & 22 & 5 & 8 & $\mathbf{3 5}$ & 19.0 & 10.5 & 5.5 & $\mathbf{3 5}$ \\
\hline Total & $\mathbf{3 8}$ & $\mathbf{2 1}$ & $\mathbf{1 1}$ & $\mathbf{7 0}$ & $\mathbf{3 8}$ & $\mathbf{2 1}$ & $\mathbf{1 1}$ & $\mathbf{7 0}$ \\
\hline
\end{tabular}

Chi-Square Value: 8.98 Degrees of Freedom: 2

Critical Value: 5.99

Table 15: Chi-Square Results 8 Cluster Solution Level II/2, Clusters 8-1 and 8-4

\begin{tabular}{|c|c|c|c|c|c|c|c|c|}
\hline Level II/2 & \multicolumn{3}{|c|}{ Observed Frequency } & Total & \multicolumn{3}{c|}{ Expected Frequency } & Total \\
\hline & Bone & $\begin{array}{c}\text { Chips and } \\
\text { Flakes }\end{array}$ & $\begin{array}{c}\text { Stone } \\
\text { Tools }\end{array}$ & & Bone & $\begin{array}{c}\text { Chips and } \\
\text { Flakes }\end{array}$ & $\begin{array}{c}\text { Stone } \\
\text { Tools }\end{array}$ & \\
\hline Cluster 8-1 & 16 & 16 & 3 & $\mathbf{3 5}$ & 16.5 & 13.7 & 4.8 & $\mathbf{3 5}$ \\
\hline Cluster 8-5 & 8 & 4 & 4 & $\mathbf{1 6}$ & 7.5 & 6.3 & 2.2 & $\mathbf{1 6}$ \\
\hline Total & $\mathbf{2 4}$ & $\mathbf{2 0}$ & $\mathbf{7}$ & $\mathbf{5 1}$ & $\mathbf{2 4}$ & $\mathbf{2 0}$ & $\mathbf{7}$ & $\mathbf{5 1}$ \\
\hline
\end{tabular}

Chi-Square Value: 3.40 Degrees of Freedom: 2

Critical Value: 5.99

Table 16: Chi-Square Results 8 Cluster Solution Level II/2, Clusters 8-1 and 8-5 


\begin{tabular}{|c|c|c|c|c|c|c|c|c|}
\hline Level II/2 & \multicolumn{3}{|c|}{ Observed Frequency } & Total & \multicolumn{3}{c|}{ Expected Frequency } & Total \\
\hline & Bone & $\begin{array}{c}\text { Chips and } \\
\text { Flakes }\end{array}$ & $\begin{array}{c}\text { Stone } \\
\text { Tools }\end{array}$ & & Bone & $\begin{array}{c}\text { Chips and } \\
\text { Flakes }\end{array}$ & $\begin{array}{c}\text { Stone } \\
\text { Tools }\end{array}$ & \\
\hline Cluster 8-1 & 16 & 16 & 3 & $\mathbf{3 5}$ & 17.1 & 15.3 & 2.7 & $\mathbf{3 5}$ \\
\hline Cluster 8-6 & 3 & 1 & 0 & $\mathbf{4}$ & 1.9 & 1.7 & 0.3 & $\mathbf{4}$ \\
\hline Total & $\mathbf{1 9}$ & $\mathbf{1 7}$ & $\mathbf{3}$ & $\mathbf{3 9}$ & $\mathbf{1 9}$ & $\mathbf{1 7}$ & $\mathbf{3}$ & $\mathbf{3 9}$ \\
\hline
\end{tabular}

Chi-Square Value: 1.33 Degrees of Freedom: 2

Critical Value: 5.99

Table 17: Chi-Square Results 8 Cluster Solution Level II/2, Clusters 8-1 and 8-6

\begin{tabular}{|c|c|c|c|c|c|c|c|c|}
\hline Level II/2 & \multicolumn{3}{|c|}{ Observed Frequency } & Total & \multicolumn{3}{|c|}{ Expected Frequency } & Total \\
\hline & Bone & $\begin{array}{c}\text { Chips and } \\
\text { Flakes }\end{array}$ & $\begin{array}{c}\text { Stone } \\
\text { Tools }\end{array}$ & & Bone & $\begin{array}{c}\text { Chips and } \\
\text { Flakes }\end{array}$ & $\begin{array}{c}\text { Stone } \\
\text { Tools }\end{array}$ & \\
\hline Cluster 8-1 & 16 & 16 & 3 & $\mathbf{3 5}$ & 19.8 & 13.0 & 2.3 & $\mathbf{3 5}$ \\
\hline Cluster 8-7 & 19 & 7 & 1 & $\mathbf{2 7}$ & 15.2 & 10.0 & 1.7 & $\mathbf{2 7}$ \\
\hline Total & $\mathbf{3 5}$ & $\mathbf{2 3}$ & $\mathbf{4}$ & $\mathbf{6 2}$ & $\mathbf{3 5}$ & $\mathbf{2 3}$ & $\mathbf{4}$ & $\mathbf{6 2}$ \\
\hline
\end{tabular}

Chi-Square Value: 3.81 Degrees of Freedom: 2

Critical Value: 5.99

Table 18: Chi-Square Results 8 Cluster Solution Level II/2, Clusters 8-1 and 8-7

\begin{tabular}{|c|c|c|c|c|c|c|c|c|}
\hline Level II/2 & \multicolumn{3}{|c|}{ Observed Frequency } & Total & \multicolumn{3}{c|}{ Expected Frequency } & Total \\
\hline & Bone & $\begin{array}{c}\text { Chips and } \\
\text { Flakes }\end{array}$ & $\begin{array}{c}\text { Stone } \\
\text { Tools }\end{array}$ & & Bone & $\begin{array}{c}\text { Chips and } \\
\text { Flakes }\end{array}$ & $\begin{array}{c}\text { Stone } \\
\text { Tools }\end{array}$ & \\
\hline Cluster 8-1 & 16 & 16 & 3 & $\mathbf{3 5}$ & 17.8 & 13.7 & 3.4 & $\mathbf{3 5}$ \\
\hline Cluster 8-8 & 10 & 4 & 2 & $\mathbf{1 6}$ & 8.2 & 6.3 & 1.6 & $\mathbf{1 6}$ \\
\hline Total & $\mathbf{2 6}$ & $\mathbf{2 0}$ & $\mathbf{5}$ & $\mathbf{5 1}$ & $\mathbf{2 6}$ & $\mathbf{2 0}$ & $\mathbf{5}$ & $\mathbf{5 1}$ \\
\hline
\end{tabular}

Chi-Square Value: 1.98 Degrees of Freedom: 2

Critical Value: 5.99

Table 19: Chi-Square Results 8 Cluster Solution Level II/2, Clusters 8-1 and 8-8

\begin{tabular}{|c|c|c|c|c|c|c|c|c|}
\hline Level II/2 & \multicolumn{3}{|c|}{ Observed Frequency } & Total & \multicolumn{3}{c|}{ Expected Frequency } & Total \\
\hline & Bone & $\begin{array}{c}\text { Chips and } \\
\text { Flakes }\end{array}$ & $\begin{array}{c}\text { Stone } \\
\text { Tools }\end{array}$ & & Bone & $\begin{array}{c}\text { Chips and } \\
\text { Flakes }\end{array}$ & $\begin{array}{c}\text { Stone } \\
\text { Tools }\end{array}$ & \\
\hline Cluster 8-2 & 23 & 6 & 2 & $\mathbf{3 1}$ & 18.7 & 7.1 & 5.2 & $\mathbf{3 1}$ \\
\hline Cluster 8-3 & 6 & 5 & 6 & $\mathbf{1 7}$ & 10.3 & 3.9 & 2.8 & $\mathbf{1 7}$ \\
\hline Total & $\mathbf{2 9}$ & $\mathbf{1 1}$ & $\mathbf{8}$ & $\mathbf{4 8}$ & $\mathbf{2 9}$ & $\mathbf{1 1}$ & $\mathbf{8}$ & $\mathbf{4 8}$ \\
\hline
\end{tabular}

Chi-Square Value: 8.71 Degrees of Freedom: 2

Critical Value: 5.99

Table 20: Chi-Square Results 8 Cluster Solution Level II/2, Clusters 8-2 and 8-3 


\begin{tabular}{|c|c|c|c|c|c|c|c|c|}
\hline Level II/2 & \multicolumn{3}{|c|}{ Observed Frequency } & Total & \multicolumn{3}{c|}{ Expected Frequency } & Total \\
\hline & Bone & $\begin{array}{c}\text { Chips and } \\
\text { Flakes }\end{array}$ & $\begin{array}{c}\text { Stone } \\
\text { Tools }\end{array}$ & & Bone & $\begin{array}{c}\text { Chips and } \\
\text { Flakes }\end{array}$ & $\begin{array}{c}\text { Stone } \\
\text { Tools }\end{array}$ & \\
\hline Cluster 8-2 & 23 & 6 & 2 & $\mathbf{3 1}$ & 21.1 & 5.2 & 4.7 & $\mathbf{3 1}$ \\
\hline Cluster 8-4 & 22 & 5 & 8 & $\mathbf{3 5}$ & 23.9 & 5.8 & 5.3 & $\mathbf{3 5}$ \\
\hline Total & $\mathbf{4 5}$ & $\mathbf{1 1}$ & $\mathbf{1 0}$ & $\mathbf{6 6}$ & $\mathbf{4 5}$ & $\mathbf{1 1}$ & $\mathbf{1 0}$ & $\mathbf{6 6}$ \\
\hline
\end{tabular}

Chi-Square Value: 3.48 Degrees of Freedom: 2

Critical Value: 5.99

Table 21: Chi-Square Results 8 Cluster Solution Level II/2, Clusters 8-2 and 8-4

\begin{tabular}{|c|c|c|c|c|c|c|c|c|}
\hline Level II/2 & \multicolumn{3}{|c|}{ Observed Frequency } & Total & \multicolumn{3}{c|}{ Expected Frequency } & Total \\
\hline & Bone & $\begin{array}{c}\text { Chips and } \\
\text { Flakes }\end{array}$ & $\begin{array}{c}\text { Stone } \\
\text { Tools }\end{array}$ & & Bone & $\begin{array}{c}\text { Chips and } \\
\text { Flakes }\end{array}$ & $\begin{array}{c}\text { Stone } \\
\text { Tools }\end{array}$ & \\
\hline Cluster 8-2 & 23 & 6 & 2 & $\mathbf{3 1}$ & 20.4 & 6.6 & 4.0 & $\mathbf{3 1}$ \\
\hline Cluster 8-5 & 8 & 4 & 4 & $\mathbf{1 6}$ & 10.6 & 3.4 & 2.0 & $\mathbf{1 6}$ \\
\hline Total & $\mathbf{3 1}$ & $\mathbf{1 0}$ & $\mathbf{6}$ & $\mathbf{4 7}$ & $\mathbf{3 1}$ & $\mathbf{1 0}$ & $\mathbf{6}$ & $\mathbf{4 7}$ \\
\hline
\end{tabular}

Chi-Square Value: 3.94 Degrees of Freedom: 2

Critical Value: 5.99

Table 22: Chi-Square Results 8 Cluster Solution Level II/2, Clusters 8-2 and 8-5

\begin{tabular}{|c|c|c|c|c|c|c|c|c|}
\hline Level II/2 & \multicolumn{3}{|c|}{ Observed Frequency } & Total & \multicolumn{3}{c|}{ Expected Frequency } & Total \\
\hline & Bone & $\begin{array}{c}\text { Chips and } \\
\text { Flakes }\end{array}$ & $\begin{array}{c}\text { Stone } \\
\text { Tools }\end{array}$ & & Bone & $\begin{array}{c}\text { Chips and } \\
\text { Flakes }\end{array}$ & $\begin{array}{c}\text { Stone } \\
\text { Tools }\end{array}$ & \\
\hline Cluster 8-2 & 23 & 6 & 2 & $\mathbf{3 1}$ & 23.0 & 6.2 & 1.8 & $\mathbf{3 1}$ \\
\hline Cluster 8-6 & 3 & 1 & 0 & $\mathbf{4}$ & 3.0 & 0.8 & 0.2 & $\mathbf{4}$ \\
\hline Total & $\mathbf{2 6}$ & $\mathbf{7}$ & $\mathbf{2}$ & $\mathbf{3 5}$ & $\mathbf{2 6}$ & $\mathbf{7}$ & $\mathbf{2}$ & $\mathbf{3 5}$ \\
\hline
\end{tabular}

Chi-Square Value: 0.31 Degrees of Freedom: 2

Critical Value: 5.99

Table 23: Chi-Square Results 8 Cluster Solution Level II/2, Clusters 8-2 and 8-6

\begin{tabular}{|c|c|c|c|c|c|c|c|c|}
\hline Level II/2 & \multicolumn{3}{|c|}{ Observed Frequency } & Total & \multicolumn{3}{|c|}{ Expected Frequency } & Total \\
\hline & Bone & $\begin{array}{c}\text { Chips and } \\
\text { Flakes }\end{array}$ & $\begin{array}{c}\text { Stone } \\
\text { Tools }\end{array}$ & & Bone & $\begin{array}{c}\text { Chips and } \\
\text { Flakes }\end{array}$ & $\begin{array}{c}\text { Stone } \\
\text { Tools }\end{array}$ & \\
\hline Cluster 8-2 & 23 & 6 & 2 & $\mathbf{3 1}$ & 22.4 & 6.9 & 1.6 & $\mathbf{3 1}$ \\
\hline Cluster 8-7 & 19 & 7 & 1 & $\mathbf{2 7}$ & 19.6 & 6.1 & 1.4 & $\mathbf{2 7}$ \\
\hline Total & $\mathbf{4 2}$ & $\mathbf{1 3}$ & $\mathbf{3}$ & $\mathbf{5 8}$ & $\mathbf{4 2}$ & $\mathbf{1 3}$ & $\mathbf{3}$ & $\mathbf{5 8}$ \\
\hline
\end{tabular}

Chi-Square Value: 0.52 Degrees of Freedom: 2

Critical Value: 5.99

Table 24: Chi-Square Results 8 Cluster Solution Level II/2, Clusters 8-2 and 8-7 


\begin{tabular}{|c|c|c|c|c|c|c|c|c|}
\hline Level II/2 & \multicolumn{3}{|c|}{ Observed Frequency } & Total & \multicolumn{3}{c|}{ Expected Frequency } & Total \\
\hline & Bone & $\begin{array}{c}\text { Chips and } \\
\text { Flakes }\end{array}$ & $\begin{array}{c}\text { Stone } \\
\text { Tools }\end{array}$ & & Bone & $\begin{array}{c}\text { Chips and } \\
\text { Flakes }\end{array}$ & $\begin{array}{c}\text { Stone } \\
\text { Tools }\end{array}$ & \\
\hline Cluster 8-2 & 23 & 6 & 2 & $\mathbf{3 1}$ & 21.8 & 6.6 & 2.6 & $\mathbf{3 1}$ \\
\hline Cluster 8-8 & 10 & 4 & 2 & $\mathbf{1 6}$ & 11.2 & 3.4 & 1.4 & $\mathbf{1 6}$ \\
\hline Total & $\mathbf{3 3}$ & $\mathbf{1 0}$ & $\mathbf{4}$ & $\mathbf{4 7}$ & $\mathbf{3 3}$ & $\mathbf{1 0}$ & $\mathbf{4}$ & $\mathbf{4 7}$ \\
\hline
\end{tabular}

Chi-Square Value: 0.82 Degrees of Freedom: 2

Critical Value: 5.99

Table 25: Chi-Square Results 8 Cluster Solution Level II/2, Clusters 8-2 and 8-8

\begin{tabular}{|c|c|c|c|c|c|c|c|c|}
\hline Level II/2 & \multicolumn{3}{|c|}{ Observed Frequency } & Total & \multicolumn{3}{c|}{ Expected Frequency } & Total \\
\hline & Bone & $\begin{array}{c}\text { Chips and } \\
\text { Flakes }\end{array}$ & $\begin{array}{c}\text { Stone } \\
\text { Tools }\end{array}$ & & Bone & $\begin{array}{c}\text { Chips and } \\
\text { Flakes }\end{array}$ & $\begin{array}{c}\text { Stone } \\
\text { Tools }\end{array}$ & \\
\hline Cluster 8-3 & 6 & 5 & 6 & $\mathbf{1 7}$ & 9.2 & 3.3 & 4.6 & $\mathbf{1 7}$ \\
\hline Cluster 8-4 & 22 & 5 & 8 & $\mathbf{3 5}$ & 18.8 & 6.7 & 9.4 & $\mathbf{3 5}$ \\
\hline Total & $\mathbf{2 8}$ & $\mathbf{1 0}$ & $\mathbf{1 4}$ & $\mathbf{5 2}$ & $\mathbf{2 8}$ & $\mathbf{1 0}$ & $\mathbf{1 4}$ & $\mathbf{5 2}$ \\
\hline
\end{tabular}

Chi-Square Value: 3.63 Degrees of Freedom: 2

Critical Value: 5.99

Table 26: Chi-Square Results 8 Cluster Solution Level II/2, Clusters 8-3 and 8-4

\begin{tabular}{|c|c|c|c|c|c|c|c|c|}
\hline Level II/2 & \multicolumn{3}{|c|}{ Observed Frequency } & Total & \multicolumn{3}{c|}{ Expected Frequency } & Total \\
\hline & Bone & $\begin{array}{c}\text { Chips and } \\
\text { Flakes }\end{array}$ & $\begin{array}{c}\text { Stone } \\
\text { Tools }\end{array}$ & & Bone & $\begin{array}{c}\text { Chips and } \\
\text { Flakes }\end{array}$ & $\begin{array}{c}\text { Stone } \\
\text { Tools }\end{array}$ & \\
\hline Cluster 8-3 & 6 & 5 & 6 & $\mathbf{1 7}$ & 7.2 & 4.6 & 5.2 & $\mathbf{1 7}$ \\
\hline Cluster 8-5 & 8 & 4 & 4 & $\mathbf{1 6}$ & 6.8 & 4.4 & 4.8 & $\mathbf{1 6}$ \\
\hline Total & $\mathbf{1 4}$ & $\mathbf{9}$ & $\mathbf{1 0}$ & $\mathbf{3 3}$ & $\mathbf{1 4}$ & $\mathbf{9}$ & $\mathbf{1 0}$ & $\mathbf{3 3}$ \\
\hline
\end{tabular}

Chi-Square Value: 0.77 Degrees of Freedom: 2

Critical Value: 5.99

Table 27: Chi-Square Results 8 Cluster Solution Level II/2, Clusters 8-3 and 8-5

\begin{tabular}{|c|c|c|c|c|c|c|c|c|}
\hline Level II/2 & \multicolumn{3}{|c|}{ Observed Frequency } & Total & \multicolumn{3}{c|}{ Expected Frequency } & Total \\
\hline & Bone & $\begin{array}{c}\text { Chips and } \\
\text { Flakes }\end{array}$ & $\begin{array}{c}\text { Stone } \\
\text { Tools }\end{array}$ & & Bone & $\begin{array}{c}\text { Chips and } \\
\text { Flakes }\end{array}$ & $\begin{array}{c}\text { Stone } \\
\text { Tools }\end{array}$ & \\
\hline Cluster 8-3 & 6 & 5 & 6 & $\mathbf{1 7}$ & 7.3 & 4.9 & 4.9 & $\mathbf{1 7}$ \\
\hline Cluster 8-6 & 3 & 1 & 0 & $\mathbf{4}$ & 1.7 & 1.1 & 1.1 & $\mathbf{4}$ \\
\hline Total & $\mathbf{9}$ & $\mathbf{6}$ & $\mathbf{6}$ & $\mathbf{2 1}$ & $\mathbf{9}$ & $\mathbf{6}$ & $\mathbf{6}$ & $\mathbf{2 1}$ \\
\hline
\end{tabular}

Chi-Square Value: 2.63 Degrees of Freedom: 2

Critical Value: 5.99

Table 28: Chi-Square Results 8 Cluster Solution Level II/2, Clusters 8-3 and 8-6 


\begin{tabular}{|c|c|c|c|c|c|c|c|c|}
\hline Level II/2 & \multicolumn{3}{|c|}{ Observed Frequency } & Total & \multicolumn{3}{c|}{ Expected Frequency } & Total \\
\hline & Bone & $\begin{array}{c}\text { Chips and } \\
\text { Flakes }\end{array}$ & $\begin{array}{c}\text { Stone } \\
\text { Tools }\end{array}$ & & Bone & $\begin{array}{c}\text { Chips and } \\
\text { Flakes }\end{array}$ & $\begin{array}{c}\text { Stone } \\
\text { Tools }\end{array}$ & \\
\hline Cluster 8-3 & 6 & 5 & 6 & $\mathbf{1 7}$ & 9.7 & 4.6 & 2.7 & $\mathbf{1 7}$ \\
\hline Cluster 8-7 & 19 & 7 & 1 & $\mathbf{2 7}$ & 15.3 & 7.4 & 4.3 & $\mathbf{2 7}$ \\
\hline Total & $\mathbf{2 5}$ & $\mathbf{1 2}$ & $\mathbf{7}$ & $\mathbf{4 4}$ & $\mathbf{2 5}$ & $\mathbf{1 2}$ & $\mathbf{7}$ & $\mathbf{4 4}$ \\
\hline
\end{tabular}

Chi-Square Value: 8.85 Degrees of Freedom: 2

Critical Value: 5.99

Table 29: Chi-Square Results 8 Cluster Solution Level II/2, Clusters 8-3 and 8-7

\begin{tabular}{|c|c|c|c|c|c|c|c|c|}
\hline Level II/2 & \multicolumn{3}{|c|}{ Observed Frequency } & Total & \multicolumn{3}{c|}{ Expected Frequency } & Total \\
\hline & Bone & $\begin{array}{c}\text { Chips and } \\
\text { Flakes }\end{array}$ & $\begin{array}{c}\text { Stone } \\
\text { Tools }\end{array}$ & & Bone & $\begin{array}{c}\text { Chips and } \\
\text { Flakes }\end{array}$ & $\begin{array}{c}\text { Stone } \\
\text { Tools }\end{array}$ & \\
\hline Cluster 8-3 & 6 & 5 & 6 & $\mathbf{1 7}$ & 8.2 & 4.6 & 4.1 & $\mathbf{1 7}$ \\
\hline Cluster 8-8 & 10 & 4 & 2 & $\mathbf{1 6}$ & 7.8 & 4.4 & 3.9 & $\mathbf{1 6}$ \\
\hline Total & $\mathbf{1 6}$ & $\mathbf{9}$ & $\mathbf{8}$ & $\mathbf{3 3}$ & $\mathbf{1 6}$ & $\mathbf{9}$ & $\mathbf{8}$ & $\mathbf{3 3}$ \\
\hline
\end{tabular}

Chi-Square Value: 3.08 Degrees of Freedom: 2

Critical Value: 5.99

Table 30: Chi-Square Results 8 Cluster Solution Level II/2, Clusters 8-3 and 8-8

\begin{tabular}{|c|c|c|c|c|c|c|c|c|}
\hline Level II/2 & \multicolumn{3}{|c|}{ Observed Frequency } & Total & \multicolumn{3}{c|}{ Expected Frequency } & Total \\
\hline & Bone & $\begin{array}{c}\text { Chips and } \\
\text { Flakes }\end{array}$ & $\begin{array}{c}\text { Stone } \\
\text { Tools }\end{array}$ & & Bone & $\begin{array}{c}\text { Chips and } \\
\text { Flakes }\end{array}$ & $\begin{array}{c}\text { Stone } \\
\text { Tools }\end{array}$ & \\
\hline Cluster 8-4 & 22 & 5 & 8 & $\mathbf{3 5}$ & 20.6 & 6.2 & 8.2 & $\mathbf{3 5}$ \\
\hline Cluster 8-5 & 8 & 4 & 4 & $\mathbf{1 6}$ & 9.4 & 2.8 & 3.8 & $\mathbf{1 6}$ \\
\hline Total & $\mathbf{3 0}$ & $\mathbf{9}$ & $\mathbf{1 2}$ & $\mathbf{5 1}$ & $\mathbf{3 0}$ & $\mathbf{9}$ & $\mathbf{1 2}$ & $\mathbf{5 1}$ \\
\hline
\end{tabular}

Chi-Square Value: 1.04 Degrees of Freedom: 2

Critical Value: 5.99

Table 31: Chi-Square Results 8 Cluster Solution Level II/2, Clusters 8-4 and 8-5

\begin{tabular}{|c|c|c|c|c|c|c|c|c|}
\hline Level II/2 & \multicolumn{3}{|c|}{ Observed Frequency } & Total & \multicolumn{3}{c|}{ Expected Frequency } & Total \\
\hline & Bone & $\begin{array}{c}\text { Chips and } \\
\text { Flakes }\end{array}$ & $\begin{array}{c}\text { Stone } \\
\text { Tools }\end{array}$ & & Bone & $\begin{array}{c}\text { Chips and } \\
\text { Flakes }\end{array}$ & $\begin{array}{c}\text { Stone } \\
\text { Tools }\end{array}$ & \\
\hline Cluster 8-4 & 22 & 5 & 8 & $\mathbf{3 5}$ & 22.4 & 5.4 & 7.2 & $\mathbf{3 5}$ \\
\hline Cluster 8-6 & 3 & 1 & 0 & $\mathbf{4}$ & 2.6 & 0.6 & 0.8 & $\mathbf{4}$ \\
\hline Total & $\mathbf{2 5}$ & $\mathbf{6}$ & $\mathbf{8}$ & $\mathbf{3 9}$ & $\mathbf{2 5}$ & $\mathbf{6}$ & $\mathbf{8}$ & $\mathbf{3 9}$ \\
\hline
\end{tabular}

Chi-Square Value: $1.26 \quad$ Degrees of Freedom: 2

Critical Value: 5.99

Table 32: Chi-Square Results 8 Cluster Solution Level II/2, Clusters 8-4 and 8-6 


\begin{tabular}{|c|c|c|c|c|c|c|c|c|}
\hline Level II/2 & \multicolumn{3}{|c|}{ Observed Frequency } & Total & \multicolumn{3}{|c|}{ Expected Frequency } & Total \\
\hline & Bone & $\begin{array}{c}\text { Chips and } \\
\text { Flakes }\end{array}$ & $\begin{array}{c}\text { Stone } \\
\text { Tools }\end{array}$ & & Bone & $\begin{array}{c}\text { Chips and } \\
\text { Flakes }\end{array}$ & $\begin{array}{c}\text { Stone } \\
\text { Tools }\end{array}$ & \\
\hline Cluster 8-4 & 22 & 5 & 8 & $\mathbf{3 5}$ & 23.1 & 6.8 & 5.1 & $\mathbf{3 5}$ \\
\hline Cluster 8-7 & 19 & 7 & 1 & $\mathbf{2 7}$ & 17.9 & 5.2 & 3.9 & $\mathbf{2 7}$ \\
\hline Total & $\mathbf{4 1}$ & $\mathbf{1 2}$ & $\mathbf{9}$ & $\mathbf{6 2}$ & $\mathbf{4 1}$ & $\mathbf{1 2}$ & $\mathbf{9}$ & $\mathbf{6 2}$ \\
\hline
\end{tabular}

Chi-Square Value: 5.05 Degrees of Freedom: 2

Critical Value: 5.99

Table 33: Chi-Square Results 8 Cluster Solution Level II/2, Clusters 8-4 and 8-7

\begin{tabular}{|c|c|c|c|c|c|c|c|c|}
\hline Level II/2 & \multicolumn{3}{|c|}{ Observed Frequency } & Total & \multicolumn{3}{c|}{ Expected Frequency } & Total \\
\hline & Bone & $\begin{array}{c}\text { Chips and } \\
\text { Flakes }\end{array}$ & $\begin{array}{c}\text { Stone } \\
\text { Tools }\end{array}$ & & Bone & $\begin{array}{c}\text { Chips and } \\
\text { Flakes }\end{array}$ & $\begin{array}{c}\text { Stone } \\
\text { Tools }\end{array}$ & \\
\hline Cluster 8-4 & 22 & 5 & 8 & $\mathbf{3 5}$ & 22.0 & 6.2 & 6.9 & $\mathbf{3 5}$ \\
\hline Cluster 8-8 & 10 & 4 & 2 & $\mathbf{1 6}$ & 10.0 & 2.8 & 3.1 & $\mathbf{1 6}$ \\
\hline Total & $\mathbf{3 2}$ & $\mathbf{9}$ & $\mathbf{1 0}$ & $\mathbf{5 1}$ & $\mathbf{3 2}$ & $\mathbf{9}$ & $\mathbf{1 0}$ & $\mathbf{5 1}$ \\
\hline
\end{tabular}

Chi-Square Value: 1.32 Degrees of Freedom: 2

Critical Value: 5.99

Table 34: Chi-Square Results 8 Cluster Solution Level II/2, Clusters 8-4 and 8-8

\begin{tabular}{|c|c|c|c|c|c|c|c|c|}
\hline Level II/2 & \multicolumn{3}{|c|}{ Observed Frequency } & Total & \multicolumn{3}{c|}{ Expected Frequency } & Total \\
\hline & Bone & $\begin{array}{c}\text { Chips and } \\
\text { Flakes }\end{array}$ & $\begin{array}{c}\text { Stone } \\
\text { Tools }\end{array}$ & & Bone & $\begin{array}{c}\text { Chips and } \\
\text { Flakes }\end{array}$ & $\begin{array}{c}\text { Stone } \\
\text { Tools }\end{array}$ & \\
\hline Cluster 8-5 & 8 & 4 & 4 & $\mathbf{1 6}$ & 8.8 & 4.0 & 3.2 & $\mathbf{1 6}$ \\
\hline Cluster 8-6 & 3 & 1 & 0 & $\mathbf{4}$ & 2.2 & 1.0 & 0.8 & $\mathbf{4}$ \\
\hline Total & $\mathbf{1 1}$ & $\mathbf{5}$ & $\mathbf{4}$ & $\mathbf{2 0}$ & $\mathbf{1 1}$ & $\mathbf{5}$ & $\mathbf{4}$ & $\mathbf{2 0}$ \\
\hline
\end{tabular}

Chi-Square Value: 1.36 Degrees of Freedom: 2

Critical Value: 5.99

Table 35: Chi-Square Results 8 Cluster Solution Level II/2, Clusters 8-5 and 8-6

\begin{tabular}{|c|c|c|c|c|c|c|c|c|}
\hline Level II/2 & \multicolumn{3}{|c|}{ Observed Frequency } & Total & \multicolumn{3}{c|}{ Expected Frequency } & Total \\
\hline & Bone & $\begin{array}{c}\text { Chips and } \\
\text { Flakes }\end{array}$ & $\begin{array}{c}\text { Stone } \\
\text { Tools }\end{array}$ & & Bone & $\begin{array}{c}\text { Chips and } \\
\text { Flakes }\end{array}$ & $\begin{array}{c}\text { Stone } \\
\text { Tools }\end{array}$ & \\
\hline Cluster 8-5 & 8 & 4 & 4 & $\mathbf{1 6}$ & 10.0 & 4.1 & 1.9 & $\mathbf{1 6}$ \\
\hline Cluster 8-7 & 19 & 7 & 1 & $\mathbf{2 7}$ & 17.0 & 6.9 & 3.1 & $\mathbf{2 7}$ \\
\hline Total & $\mathbf{2 7}$ & $\mathbf{1 1}$ & $\mathbf{5}$ & $\mathbf{4 3}$ & $\mathbf{2 7}$ & $\mathbf{1 1}$ & $\mathbf{5}$ & $\mathbf{4 3}$ \\
\hline
\end{tabular}

Chi-Square Value: 4.59 Degrees of Freedom: 2

Critical Value: 5.99

Table 36: Chi-Square Results 8 Cluster Solution Level II/2, Clusters 8-5 and 8-7 


\begin{tabular}{|c|c|c|c|c|c|c|c|c|}
\hline Level II/2 & \multicolumn{3}{|c|}{ Observed Frequency } & Total & \multicolumn{3}{c|}{ Expected Frequency } & Total \\
\hline & Bone & $\begin{array}{c}\text { Chips and } \\
\text { Flakes }\end{array}$ & $\begin{array}{c}\text { Stone } \\
\text { Tools }\end{array}$ & & Bone & $\begin{array}{c}\text { Chips and } \\
\text { Flakes }\end{array}$ & $\begin{array}{c}\text { Stone } \\
\text { Tools }\end{array}$ & \\
\hline Cluster 8-5 & 8 & 4 & 4 & $\mathbf{1 6}$ & 9.0 & 4.0 & 3.0 & $\mathbf{1 6}$ \\
\hline Cluster 8-8 & 10 & 4 & 2 & $\mathbf{1 6}$ & 9.0 & 4.0 & 3.0 & $\mathbf{1 6}$ \\
\hline Total & $\mathbf{1 8}$ & $\mathbf{8}$ & $\mathbf{6}$ & $\mathbf{3 2}$ & $\mathbf{1 8}$ & $\mathbf{8}$ & $\mathbf{6}$ & $\mathbf{3 2}$ \\
\hline
\end{tabular}

Chi-Square Value: 0.89 Degrees of Freedom: 2

Critical Value: 5.99

Table 37: Chi-Square Results 8 Cluster Solution Level II/2, Clusters 8-5 and 8-8

\begin{tabular}{|c|c|c|c|c|c|c|c|c|}
\hline Level II/2 & \multicolumn{3}{|c|}{ Observed Frequency } & Total & \multicolumn{3}{c|}{ Expected Frequency } & Total \\
\hline & Bone & $\begin{array}{c}\text { Chips and } \\
\text { Flakes }\end{array}$ & $\begin{array}{c}\text { Stone } \\
\text { Tools }\end{array}$ & & Bone & $\begin{array}{c}\text { Chips and } \\
\text { Flakes }\end{array}$ & $\begin{array}{c}\text { Stone } \\
\text { Tools }\end{array}$ & \\
\hline Cluster 8-6 & 3 & 1 & 0 & $\mathbf{4}$ & 2.8 & 1.0 & 0.1 & $\mathbf{4}$ \\
\hline Cluster 8-7 & 19 & 7 & 1 & $\mathbf{2 7}$ & 19.2 & 7.0 & 0.9 & $\mathbf{2 7}$ \\
\hline Total & $\mathbf{2 2}$ & $\mathbf{8}$ & $\mathbf{1}$ & $\mathbf{3 1}$ & $\mathbf{2 2}$ & $\mathbf{8}$ & $\mathbf{1}$ & $\mathbf{3 1}$ \\
\hline
\end{tabular}

Chi-Square Value: 0.16 Degrees of Freedom: 2

Critical Value: 5.99

Table 38: Chi-Square Results 8 Cluster Solution Level II/2, Clusters 8-6 and 8-7

\begin{tabular}{|c|c|c|c|c|c|c|c|c|}
\hline Level II/2 & \multicolumn{3}{|c|}{ Observed Frequency } & Total & \multicolumn{3}{c|}{ Expected Frequency } & Total \\
\hline & Bone & $\begin{array}{c}\text { Chips and } \\
\text { Flakes }\end{array}$ & $\begin{array}{c}\text { Stone } \\
\text { Tools }\end{array}$ & & Bone & $\begin{array}{c}\text { Chips and } \\
\text { Flakes }\end{array}$ & $\begin{array}{c}\text { Stone } \\
\text { Tools }\end{array}$ & \\
\hline Cluster 8-6 & 3 & 1 & 0 & $\mathbf{4}$ & 2.6 & 1.0 & 0.4 & $\mathbf{4}$ \\
\hline Cluster 8-8 & 10 & 4 & 2 & $\mathbf{1 6}$ & 10.4 & 4.0 & 1.6 & $\mathbf{1 6}$ \\
\hline Total & $\mathbf{1 3}$ & $\mathbf{5}$ & $\mathbf{2}$ & $\mathbf{2 0}$ & $\mathbf{1 3}$ & $\mathbf{5}$ & $\mathbf{2}$ & $\mathbf{2 0}$ \\
\hline
\end{tabular}

Chi-Square Value: 0.58 Degrees of Freedom: 2

Critical Value: 5.99

Table 39: Chi-Square Results 8 Cluster Solution Level IL/2, Clusters 8-6 and 8-8

\begin{tabular}{|c|c|c|c|c|c|c|c|c|}
\hline Level II/2 & \multicolumn{3}{|c|}{ Observed Frequency } & Total & \multicolumn{3}{c|}{ Expected Frequency } & Total \\
\hline & Bone & $\begin{array}{c}\text { Chips and } \\
\text { Flakes }\end{array}$ & $\begin{array}{c}\text { Stone } \\
\text { Tools }\end{array}$ & & Bone & $\begin{array}{c}\text { Chips and } \\
\text { Flakes }\end{array}$ & $\begin{array}{c}\text { Stone } \\
\text { Tools }\end{array}$ & \\
\hline Cluster 8-7 & 19 & 7 & 1 & $\mathbf{2 7}$ & 18.2 & 6.9 & 1.9 & $\mathbf{2 7}$ \\
\hline Cluster 8-8 & 10 & 4 & 2 & $\mathbf{1 6}$ & 10.8 & 4.1 & 1.1 & $\mathbf{1 6}$ \\
\hline Total & $\mathbf{2 9}$ & $\mathbf{1 1}$ & $\mathbf{3}$ & $\mathbf{4 3}$ & $\mathbf{2 9}$ & $\mathbf{1 1}$ & $\mathbf{3}$ & $\mathbf{4 3}$ \\
\hline
\end{tabular}

Chi-Square Value: 1.21 Degrees of Freedom: 2

Critical Value: 5.99

Table 40: Chi-Square Results 8 Cluster Solution Level II/2, Clusters 8-7 and 8-8 


\section{Chi-Square Contingency Table Results - 2 Cluster Solution Level III}

\begin{tabular}{|c|c|c|c|c|c|c|c|c|}
\hline Level III & \multicolumn{3}{|c|}{ Observed Frequency } & Total & \multicolumn{3}{|c|}{ Expected Frequency } & Total \\
\hline & Bone & $\begin{array}{c}\text { Chips and } \\
\text { Flakes }\end{array}$ & $\begin{array}{c}\text { Stone } \\
\text { Tools }\end{array}$ & & Bone & $\begin{array}{c}\text { Chips and } \\
\text { Flakes }\end{array}$ & $\begin{array}{c}\text { Stone } \\
\text { Tools }\end{array}$ & \\
\hline Cluster 2-1 & 58 & 50 & 14 & $\mathbf{1 2 2}$ & 55.6 & 43.3 & 23.1 & $\mathbf{1 2 2}$ \\
\hline Cluster 2-2 & 55 & 38 & 33 & $\mathbf{1 2 6}$ & 57.4 & 44.7 & 23.9 & $\mathbf{1 2 6}$ \\
\hline Total & $\mathbf{1 1 3}$ & $\mathbf{8 8}$ & $\mathbf{4 7}$ & $\mathbf{2 4 8}$ & $\mathbf{1 1 3}$ & $\mathbf{8 8}$ & $\mathbf{4 7}$ & $\mathbf{2 4 8}$ \\
\hline
\end{tabular}

Chi-Square Value: 9.33 Degrees of Freedom: 2

Critical Value: 5.99

Table 41: Chi-Square Results 2 Cluster Solution Level III, Clusters 2-1 and 2-2

\section{Chi-Square Contingency Table Results - 4 Cluster Solution Level III}

\begin{tabular}{|c|c|c|c|c|c|c|c|c|}
\hline Level III & \multicolumn{3}{|c|}{ Observed Frequency } & Total & \multicolumn{3}{c|}{ Expected Frequency } & Total \\
\hline & Bone & $\begin{array}{c}\text { Chips and } \\
\text { Flakes }\end{array}$ & $\begin{array}{c}\text { Stone } \\
\text { Tools }\end{array}$ & & Bone & $\begin{array}{c}\text { Chips and } \\
\text { Flakes }\end{array}$ & $\begin{array}{c}\text { Stone } \\
\text { Tools }\end{array}$ & \\
\hline Cluster 4-1 & 34 & 18 & 14 & $\mathbf{6 6}$ & 28.0 & 22.2 & 15.8 & $\mathbf{6 6}$ \\
\hline Cluster 4-2 & 19 & 24 & 16 & $\mathbf{5 9}$ & 25.0 & 19.8 & 14.2 & $\mathbf{5 9}$ \\
\hline Total & $\mathbf{5 3}$ & $\mathbf{4 2}$ & $\mathbf{3 0}$ & $\mathbf{1 2 5}$ & $\mathbf{5 3}$ & $\mathbf{4 2}$ & $\mathbf{3 0}$ & $\mathbf{1 2 5}$ \\
\hline
\end{tabular}

Chi-Square Value: 4.86 Degrees of Freedom: 2

Critical Value: 5.99

Table 42: Chi-Square Results 4 Cluster Solution Level III, Clusters 4-1 and 4-2

\begin{tabular}{|c|c|c|c|c|c|c|c|c|}
\hline Level III & \multicolumn{3}{|c|}{ Observed Frequency } & Total & \multicolumn{3}{c|}{ Expected Frequency } & Total \\
\hline & Bone & $\begin{array}{c}\text { Chips and } \\
\text { Flakes }\end{array}$ & $\begin{array}{c}\text { Stone } \\
\text { Tools }\end{array}$ & & Bone & $\begin{array}{c}\text { Chips and } \\
\text { Flakes }\end{array}$ & $\begin{array}{c}\text { Stone } \\
\text { Tools }\end{array}$ & \\
\hline Cluster 4-1 & 34 & 18 & 14 & $\mathbf{6 6}$ & 35.6 & 16.2 & 14.1 & $\mathbf{6 6}$ \\
\hline Cluster 4-3 & 34 & 13 & 13 & $\mathbf{6 0}$ & 32.4 & 14.8 & 12.9 & $\mathbf{6 0}$ \\
\hline Total & $\mathbf{6 8}$ & $\mathbf{3 1}$ & $\mathbf{2 7}$ & $\mathbf{1 2 6}$ & $\mathbf{6 8}$ & $\mathbf{3 1}$ & $\mathbf{2 7}$ & $\mathbf{1 2 6}$ \\
\hline
\end{tabular}

Chi-Square Value: 0.56 Degrees of Freedom: 2

Critical Value: 5.99

Table 43: Chi-Square Results 4 Cluster Solution Level III, Clusters 4-1 and 4-3 


\begin{tabular}{|c|c|c|c|c|c|c|c|c|}
\hline Level III & \multicolumn{3}{|c|}{ Observed Frequency } & Total & \multicolumn{3}{c|}{ Expected Frequency } & Total \\
\hline & Bone & $\begin{array}{c}\text { Chips and } \\
\text { Flakes }\end{array}$ & $\begin{array}{c}\text { Stone } \\
\text { Tools }\end{array}$ & & Bone & $\begin{array}{c}\text { Chips and } \\
\text { Flakes }\end{array}$ & $\begin{array}{c}\text { Stone } \\
\text { Tools }\end{array}$ & \\
\hline Cluster 4-1 & 34 & 18 & 14 & $\mathbf{6 6}$ & 30.7 & 26.1 & 9.2 & $\mathbf{6 6}$ \\
\hline Cluster 4-4 & 26 & 33 & 4 & $\mathbf{6 3}$ & 29.3 & 24.9 & 8.8 & $\mathbf{6 3}$ \\
\hline Total & $\mathbf{6 0}$ & $\mathbf{5 1}$ & $\mathbf{1 8}$ & $\mathbf{1 2 9}$ & $\mathbf{6 0}$ & $\mathbf{5 1}$ & $\mathbf{1 8}$ & $\mathbf{1 2 9}$ \\
\hline
\end{tabular}

Chi-Square Value: 10.97 Degrees of Freedom: 2

Critical Value: 5.99

Table 44: Chi-Square Results 4 Cluster Solution Level III, Clusters 4-1 and 4-4

\begin{tabular}{|c|c|c|c|c|c|c|c|c|}
\hline Level III & \multicolumn{3}{|c|}{ Observed Frequency } & Total & \multicolumn{3}{|c|}{ Expected Frequency } & Total \\
\hline & Bone & $\begin{array}{c}\text { Chips and } \\
\text { Flakes }\end{array}$ & $\begin{array}{c}\text { Stone } \\
\text { Tools }\end{array}$ & & Bone & $\begin{array}{c}\text { Chips and } \\
\text { Flakes }\end{array}$ & $\begin{array}{c}\text { Stone } \\
\text { Tools }\end{array}$ & \\
\hline Cluster 4-2 & 19 & 24 & 16 & $\mathbf{5 9}$ & 26.3 & 18.3 & 14.4 & $\mathbf{5 9}$ \\
\hline Cluster 4-3 & 34 & 13 & 13 & $\mathbf{6 0}$ & 26.7 & 18.7 & 14.6 & $\mathbf{6 0}$ \\
\hline Total & $\mathbf{5 3}$ & $\mathbf{3 7}$ & $\mathbf{2 9}$ & $\mathbf{1 1 9}$ & $\mathbf{5 3}$ & $\mathbf{3 7}$ & $\mathbf{2 9}$ & $\mathbf{1 1 9}$ \\
\hline
\end{tabular}

Chi-Square Value: 7.82 Degrees of Freedom: 2

Critical Value: 5.99

Table 45: Chi-Square Results 4 Cluster Solution Level III, Clusters 4-2 and 4-3

\begin{tabular}{|c|c|c|c|c|c|c|c|c|}
\hline Level III & \multicolumn{3}{|c|}{ Observed Frequency } & Total & \multicolumn{3}{c|}{ Expected Frequency } & Total \\
\hline & Bone & $\begin{array}{c}\text { Chips and } \\
\text { Flakes }\end{array}$ & $\begin{array}{c}\text { Stone } \\
\text { Tools }\end{array}$ & & Bone & $\begin{array}{c}\text { Chips and } \\
\text { Flakes }\end{array}$ & $\begin{array}{c}\text { Stone } \\
\text { Tools }\end{array}$ & \\
\hline Cluster 4-2 & 19 & 24 & 16 & $\mathbf{5 9}$ & 21.8 & 27.6 & 9.7 & $\mathbf{5 9}$ \\
\hline Cluster 4-4 & 26 & 33 & 4 & $\mathbf{6 3}$ & 23.2 & 29.4 & 10.3 & $\mathbf{6 3}$ \\
\hline Total & $\mathbf{4 5}$ & $\mathbf{5 7}$ & $\mathbf{2 0}$ & $\mathbf{1 2 2}$ & $\mathbf{4 5}$ & $\mathbf{5 7}$ & $\mathbf{2 0}$ & $\mathbf{1 2 2}$ \\
\hline
\end{tabular}

Chi-Square Value: 9.59 Degrees of Freedom: 2

Critical Value: 5.99

Table 46: Chi-Square Results 4 Cluster Solution Level III, Clusters 4-2 and 4-4

\begin{tabular}{|c|c|c|c|c|c|c|c|c|}
\hline Level III & \multicolumn{3}{|c|}{ Observed Frequency } & Total & \multicolumn{3}{c|}{ Expected Frequency } & Total \\
\hline & Bone & $\begin{array}{c}\text { Chips and } \\
\text { Flakes }\end{array}$ & $\begin{array}{c}\text { Stone } \\
\text { Tools }\end{array}$ & & Bone & $\begin{array}{c}\text { Chips and } \\
\text { Flakes }\end{array}$ & $\begin{array}{c}\text { Stone } \\
\text { Tools }\end{array}$ & \\
\hline Cluster 4-3 & 34 & 13 & 13 & $\mathbf{6 0}$ & 29.3 & 22.4 & 8.3 & $\mathbf{6 0}$ \\
\hline Cluster 4-4 & 26 & 33 & 4 & $\mathbf{6 3}$ & 30.7 & 23.6 & 8.7 & $\mathbf{6 3}$ \\
\hline Total & $\mathbf{6 0}$ & $\mathbf{4 6}$ & $\mathbf{1 7}$ & $\mathbf{1 2 3}$ & $\mathbf{6 0}$ & $\mathbf{4 6}$ & $\mathbf{1 7}$ & $\mathbf{1 2 3}$ \\
\hline
\end{tabular}

Chi-Square Value: 14.46 Degrees of Freedom: 2

Critical Value: 5.99

Table 47: Chi-Square Results 4 Cluster Solution Level III, Clusters 4-3 and 4-4 


\section{Chi-Square Contingency Table Results - 6 Cluster Solution Level III}

\begin{tabular}{|l|l|l|l|l|l|l|l|l|}
\hline Level III & \multicolumn{3}{|c|}{ Observed Frequency } & Total & \multicolumn{3}{|c|}{ Expected Frequency } & Total \\
\hline & Bone & $\begin{array}{l}\text { Chips and } \\
\text { Flakes }\end{array}$ & $\begin{array}{l}\text { Stone } \\
\text { Tools }\end{array}$ & & Bone & $\begin{array}{l}\text { Chips and } \\
\text { Flakes }\end{array}$ & $\begin{array}{l}\text { Stone } \\
\text { Tools }\end{array}$ & \\
\hline Cluster 6-1 & 24 & 12 & 9 & $\mathbf{4 5}$ & 18.6 & 15.5 & 10.9 & $\mathbf{4 5}$ \\
\hline Cluster 6-2 & 17 & 22 & 15 & $\mathbf{5 4}$ & 22.4 & 18.5 & 13.1 & $\mathbf{5 4}$ \\
\hline Total & $\mathbf{4 1}$ & $\mathbf{3 4}$ & $\mathbf{2 4}$ & $\mathbf{9 9}$ & $\mathbf{4 1}$ & $\mathbf{3 4}$ & $\mathbf{2 4}$ & $\mathbf{9 9}$ \\
\hline
\end{tabular}

Chi-Square Value: 4.86 Degrees of Freedom: 2

Critical Value: 5.99

Table 48: Chi-Square Results 6 Cluster Solution Level III, Clusters 6-1 and 6-2

\begin{tabular}{|l|l|l|l|l|l|l|l|l|}
\hline Level III & \multicolumn{3}{|c|}{ Observed Frequency } & Total & \multicolumn{3}{c|}{ Expected Frequency } & Total \\
\hline & Bone & $\begin{array}{l}\text { Chips and } \\
\text { Flakes }\end{array}$ & $\begin{array}{l}\text { Stone } \\
\text { Tools }\end{array}$ & & Bone & $\begin{array}{l}\text { Chips and } \\
\text { Flakes }\end{array}$ & $\begin{array}{l}\text { Stone } \\
\text { Tools }\end{array}$ & \\
\hline Cluster 6-1 & 24 & 12 & 9 & $\mathbf{4 5}$ & 18.3 & 14.7 & 12.0 & $\mathbf{4 5}$ \\
\hline Cluster 6-3 & 11 & 16 & 14 & $\mathbf{4 1}$ & 16.7 & 13.3 & 11.0 & $\mathbf{4 1}$ \\
\hline Total & $\mathbf{3 5}$ & $\mathbf{2 8}$ & $\mathbf{2 3}$ & $\mathbf{8 6}$ & $\mathbf{3 5}$ & $\mathbf{2 8}$ & $\mathbf{2 3}$ & $\mathbf{8 6}$ \\
\hline
\end{tabular}

Chi-Square Value: 6.31 Degrees of Freedom: 2

Critical Value: 5.99

Table 49: Chi-Square Results 6 Cluster Solution Level III, Clusters 6-1 and 6-3

\begin{tabular}{|l|l|l|l|l|l|l|l|l|}
\hline Level III & \multicolumn{3}{|c|}{ Observed Frequency } & Total & \multicolumn{3}{c|}{ Expected Frequency } & Total \\
\hline & Bone & $\begin{array}{l}\text { Chips and } \\
\text { Flakes }\end{array}$ & $\begin{array}{l}\text { Stone } \\
\text { Tools }\end{array}$ & & Bone & $\begin{array}{l}\text { Chips and } \\
\text { Flakes }\end{array}$ & $\begin{array}{l}\text { Stone } \\
\text { Tools }\end{array}$ & \\
\hline Cluster 6-1 & 24 & 12 & 9 & $\mathbf{4 5}$ & 27.6 & 12.6 & 4.8 & $\mathbf{4 5}$ \\
\hline Cluster 6-4 & 33 & 14 & 1 & $\mathbf{4 8}$ & 29.4 & 13.4 & 5.2 & $\mathbf{4 8}$ \\
\hline Total & $\mathbf{5 7}$ & $\mathbf{2 6}$ & $\mathbf{1 0}$ & $\mathbf{9 3}$ & $\mathbf{5 7}$ & $\mathbf{2 6}$ & $\mathbf{1 0}$ & $\mathbf{9 3}$ \\
\hline
\end{tabular}

Chi-Square Value: $7.89 \quad$ Degrees of Freedom: 2

Critical Value: 5.99

Table 50: Chi-Square Results 6 Cluster Solution Level III, Clusters 6-1 and 6-4 


\begin{tabular}{|c|c|c|c|c|c|c|c|c|}
\hline Level III & \multicolumn{3}{|c|}{ Observed Frequency } & Total & \multicolumn{3}{c|}{ Expected Frequency } & Total \\
\hline & Bone & $\begin{array}{c}\text { Chips and } \\
\text { Flakes }\end{array}$ & $\begin{array}{c}\text { Stone } \\
\text { Tools }\end{array}$ & & Bone & $\begin{array}{c}\text { Chips and } \\
\text { Flakes }\end{array}$ & $\begin{array}{c}\text { Stone } \\
\text { Tools }\end{array}$ & \\
\hline Cluster 6-1 & 24 & 12 & 9 & $\mathbf{4 5}$ & 25.9 & 11.1 & 7.9 & $\mathbf{4 5}$ \\
\hline Cluster 6-5 & 25 & 9 & 6 & $\mathbf{4 0}$ & 23.1 & 9.9 & 7.1 & $\mathbf{4 0}$ \\
\hline Total & $\mathbf{4 9}$ & $\mathbf{2 1}$ & $\mathbf{1 5}$ & $\mathbf{8 5}$ & $\mathbf{4 9}$ & $\mathbf{2 1}$ & $\mathbf{1 5}$ & $\mathbf{8 5}$ \\
\hline
\end{tabular}

Chi-Square Value: 0.76 Degrees of Freedom: 2

Critical Value: 5.99

Table 51: Chi-Square Results 6 Cluster Solution Level III, Clusters 6-1 and 6-5

\begin{tabular}{|c|c|c|c|c|c|c|c|c|}
\hline Level III & \multicolumn{3}{|c|}{ Observed Frequency } & Total & \multicolumn{3}{c|}{ Expected Frequency } & Total \\
\hline & Bone & $\begin{array}{c}\text { Chips and } \\
\text { Flakes }\end{array}$ & $\begin{array}{c}\text { Stone } \\
\text { Tools }\end{array}$ & & Bone & $\begin{array}{c}\text { Chips and } \\
\text { Flakes }\end{array}$ & $\begin{array}{c}\text { Stone } \\
\text { Tools }\end{array}$ & \\
\hline Cluster 6-1 & 24 & 12 & 9 & $\mathbf{4 5}$ & 18.7 & 18.7 & 7.6 & $\mathbf{4 5}$ \\
\hline Cluster 6-6 & 3 & 15 & 2 & $\mathbf{2 0}$ & 8.3 & 8.3 & 3.4 & $\mathbf{2 0}$ \\
\hline Total & $\mathbf{2 7}$ & $\mathbf{2 7}$ & $\mathbf{1 1}$ & $\mathbf{6 5}$ & $\mathbf{2 7}$ & $\mathbf{2 7}$ & $\mathbf{1 1}$ & $\mathbf{6 5}$ \\
\hline
\end{tabular}

Chi-Square Value: 13.50 Degrees of Freedom: 2

Critical Value: 5.99

Table 52: Chi-Square Results 6 Cluster Solution Level III, Clusters 6-1 and 6-6

\begin{tabular}{|c|c|c|c|c|c|c|c|c|}
\hline Level III & \multicolumn{3}{|c|}{ Observed Frequency } & Total & \multicolumn{3}{c|}{ Expected Frequency } & Total \\
\hline & Bone & $\begin{array}{c}\text { Chips and } \\
\text { Flakes }\end{array}$ & $\begin{array}{c}\text { Stone } \\
\text { Tools }\end{array}$ & & Bone & $\begin{array}{c}\text { Chips and } \\
\text { Flakes }\end{array}$ & $\begin{array}{c}\text { Stone } \\
\text { Tools }\end{array}$ & \\
\hline Cluster 6-2 & 17 & 22 & 15 & $\mathbf{5 4}$ & 15.9 & 21.6 & 16.5 & $\mathbf{5 4}$ \\
\hline Cluster 6-3 & 11 & 16 & 14 & $\mathbf{4 1}$ & 12.1 & 16.4 & 12.5 & $\mathbf{4 1}$ \\
\hline Total & $\mathbf{2 8}$ & $\mathbf{3 8}$ & $\mathbf{2 9}$ & $\mathbf{9 5}$ & $\mathbf{2 8}$ & $\mathbf{3 8}$ & $\mathbf{2 9}$ & $\mathbf{9 5}$ \\
\hline
\end{tabular}

Chi-Square Value: 0.50 Degrees of Freedom: 2

Critical Value: 5.99

Table 53: Chi-Square Results 6 Cluster Solution Level III, Clusters 6-2 and 6-3

\begin{tabular}{|c|c|c|c|c|c|c|c|c|}
\hline Level III & \multicolumn{3}{|c|}{ Observed Frequency } & Total & \multicolumn{3}{c|}{ Expected Frequency } & Total \\
\hline & Bone & $\begin{array}{c}\text { Chips and } \\
\text { Flakes }\end{array}$ & $\begin{array}{c}\text { Stone } \\
\text { Tools }\end{array}$ & & Bone & $\begin{array}{c}\text { Chips and } \\
\text { Flakes }\end{array}$ & $\begin{array}{c}\text { Stone } \\
\text { Tools }\end{array}$ & \\
\hline Cluster 6-2 & 17 & 22 & 15 & $\mathbf{5 4}$ & 26.5 & 19.1 & 8.5 & $\mathbf{5 4}$ \\
\hline Cluster 6-4 & 33 & 14 & 1 & $\mathbf{4 8}$ & 23.5 & 16.9 & 7.5 & $\mathbf{4 8}$ \\
\hline Total & $\mathbf{5 0}$ & $\mathbf{3 6}$ & $\mathbf{1 6}$ & $\mathbf{1 0 2}$ & $\mathbf{5 0}$ & $\mathbf{3 6}$ & $\mathbf{1 6}$ & $\mathbf{1 0 2}$ \\
\hline
\end{tabular}

Chi-Square Value: 18.86 Degrees of Freedom: 2

Critical Value: 5.99

Table 54: Chi-Square Results 6 Cluster Solution Level III, Clusters 6-2 and 6-4 


\begin{tabular}{|c|c|c|c|c|c|c|c|c|}
\hline Level III & \multicolumn{3}{|c|}{ Observed Frequency } & Total & \multicolumn{3}{|c|}{ Expected Frequency } & Total \\
\hline & Bone & $\begin{array}{c}\text { Chips and } \\
\text { Flakes }\end{array}$ & $\begin{array}{c}\text { Stone } \\
\text { Tools }\end{array}$ & & Bone & $\begin{array}{c}\text { Chips and } \\
\text { Flakes }\end{array}$ & $\begin{array}{c}\text { Stone } \\
\text { Tools }\end{array}$ & \\
\hline Cluster 6-2 & 17 & 22 & 15 & $\mathbf{5 4}$ & 24.1 & 17.8 & 12.1 & $\mathbf{5 4}$ \\
\hline Cluster 6-5 & 25 & 9 & 6 & $\mathbf{4 0}$ & 17.9 & 13.2 & 8.9 & $\mathbf{4 0}$ \\
\hline Total & $\mathbf{4 2}$ & $\mathbf{3 1}$ & $\mathbf{2 1}$ & $\mathbf{9 4}$ & $\mathbf{4 2}$ & $\mathbf{3 1}$ & $\mathbf{2 1}$ & $\mathbf{9 4}$ \\
\hline
\end{tabular}

Chi-Square Value: 8.95 Degrees of Freedom: 2

Critical Value: 5.99

Table 55: Chi-Square Results 6 Cluster Solution Level III, Clusters 6-2 and 6-5

\begin{tabular}{|c|c|c|c|c|c|c|c|c|}
\hline Level III & \multicolumn{3}{|c|}{ Observed Frequency } & Total & \multicolumn{3}{c|}{ Expected Frequency } & Total \\
\hline & Bone & $\begin{array}{c}\text { Chips and } \\
\text { Flakes }\end{array}$ & $\begin{array}{c}\text { Stone } \\
\text { Tools }\end{array}$ & & Bone & $\begin{array}{c}\text { Chips and } \\
\text { Flakes }\end{array}$ & $\begin{array}{c}\text { Stone } \\
\text { Tools }\end{array}$ & \\
\hline Cluster 6-2 & 17 & 22 & 15 & $\mathbf{5 4}$ & 14.6 & 27.0 & 12.4 & $\mathbf{5 4}$ \\
\hline Cluster 6-6 & 3 & 15 & 2 & $\mathbf{2 0}$ & 5.4 & 10.0 & 4.6 & $\mathbf{2 0}$ \\
\hline Total & $\mathbf{2 0}$ & $\mathbf{3 7}$ & $\mathbf{1 7}$ & $\mathbf{7 4}$ & $\mathbf{2 0}$ & $\mathbf{3 7}$ & $\mathbf{1 7}$ & $\mathbf{7 4}$ \\
\hline
\end{tabular}

Chi-Square Value: 6.90 Degrees of Freedom: 2

Critical Value: 5.99

Table 56: Chi-Square Results 6 Cluster Solution Level III, Clusters 6-2 and 6-6

\begin{tabular}{|c|c|c|c|c|c|c|c|c|}
\hline Level III & \multicolumn{3}{|c|}{ Observed Frequency } & Total & \multicolumn{3}{c|}{ Expected Frequency } & Total \\
\hline & Bone & $\begin{array}{c}\text { Chips and } \\
\text { Flakes }\end{array}$ & $\begin{array}{c}\text { Stone } \\
\text { Tools }\end{array}$ & & Bone & $\begin{array}{c}\text { Chips and } \\
\text { Flakes }\end{array}$ & $\begin{array}{c}\text { Stone } \\
\text { Tools }\end{array}$ & \\
\hline Cluster 6-3 & 11 & 16 & 14 & $\mathbf{4 1}$ & 20.3 & 13.8 & 6.9 & $\mathbf{4 1}$ \\
\hline Cluster 6-4 & 33 & 14 & 1 & $\mathbf{4 8}$ & 23.7 & 16.2 & 8.1 & $\mathbf{4 8}$ \\
\hline Total & $\mathbf{4 4}$ & $\mathbf{3 0}$ & $\mathbf{1 5}$ & $\mathbf{8 9}$ & $\mathbf{4 4}$ & $\mathbf{3 0}$ & $\mathbf{1 5}$ & $\mathbf{8 9}$ \\
\hline
\end{tabular}

Chi-Square Value: 21.99 Degrees of Freedom: 2

Critical Value: 5.99

Table 57: Chi-Square Results 6 Cluster Solution Level III, Clusters 6-3 and 6-4

\begin{tabular}{|c|c|c|c|c|c|c|c|c|}
\hline Level III & \multicolumn{3}{|c|}{ Observed Frequency } & Total & \multicolumn{3}{c|}{ Expected Frequency } & Total \\
\hline & Bone & $\begin{array}{c}\text { Chips and } \\
\text { Flakes }\end{array}$ & $\begin{array}{c}\text { Stone } \\
\text { Tools }\end{array}$ & & Bone & $\begin{array}{c}\text { Chips and } \\
\text { Flakes }\end{array}$ & $\begin{array}{c}\text { Stone } \\
\text { Tools }\end{array}$ & \\
\hline Cluster 6-3 & 11 & 16 & 14 & $\mathbf{4 1}$ & 18.2 & 12.7 & 10.1 & $\mathbf{4 1}$ \\
\hline Cluster 6-5 & 25 & 9 & 6 & $\mathbf{4 0}$ & 17.8 & 12.3 & 9.9 & $\mathbf{4 0}$ \\
\hline Total & $\mathbf{3 6}$ & $\mathbf{2 5}$ & $\mathbf{2 0}$ & $\mathbf{8 1}$ & $\mathbf{3 6}$ & $\mathbf{2 5}$ & $\mathbf{2 0}$ & $\mathbf{8 1}$ \\
\hline
\end{tabular}

Chi-Square Value: 10.59 Degrees of Freedom: 2

Critical Value: 5.99

Table 58: Chi-Square Results 6 Cluster Solution Level III, Clusters 6-3 and 6-5 


\begin{tabular}{|c|c|c|c|c|c|c|c|c|}
\hline Level III & \multicolumn{3}{|c|}{ Observed Frequency } & Total & \multicolumn{3}{c|}{ Expected Frequency } & Total \\
\hline & Bone & $\begin{array}{c}\text { Chips and } \\
\text { Flakes }\end{array}$ & $\begin{array}{c}\text { Stone } \\
\text { Tools }\end{array}$ & & Bone & $\begin{array}{c}\text { Chips and } \\
\text { Flakes }\end{array}$ & $\begin{array}{c}\text { Stone } \\
\text { Tools }\end{array}$ & \\
\hline Cluster 6-3 & 11 & 16 & 14 & $\mathbf{4 1}$ & 9.4 & 20.8 & 10.8 & $\mathbf{4 1}$ \\
\hline Cluster 6-6 & 3 & 15 & 2 & $\mathbf{2 0}$ & 4.6 & 10.2 & 5.2 & $\mathbf{2 0}$ \\
\hline Total & $\mathbf{1 4}$ & $\mathbf{3 1}$ & $\mathbf{1 6}$ & $\mathbf{6 1}$ & $\mathbf{1 4}$ & $\mathbf{3 1}$ & $\mathbf{1 6}$ & $\mathbf{6 1}$ \\
\hline
\end{tabular}

Chi-Square Value: 7.23 Degrees of Freedom: 2

Critical Value: 5.99

Table 59: Chi-Square Results 6 Cluster Solution Level III, Clusters 6-3 and 6-6

\begin{tabular}{|c|c|c|c|c|c|c|c|c|}
\hline Level III & \multicolumn{3}{|c|}{ Observed Frequency } & Total & \multicolumn{3}{c|}{ Expected Frequency } & Total \\
\hline & Bone & $\begin{array}{c}\text { Chips and } \\
\text { Flakes }\end{array}$ & $\begin{array}{c}\text { Stone } \\
\text { Tools }\end{array}$ & & Bone & $\begin{array}{c}\text { Chips and } \\
\text { Flakes }\end{array}$ & $\begin{array}{c}\text { Stone } \\
\text { Tools }\end{array}$ & \\
\hline Cluster 6-4 & 33 & 14 & 1 & $\mathbf{4 8}$ & 31.6 & 12.5 & 3.8 & $\mathbf{4 8}$ \\
\hline Cluster 6-5 & 25 & 9 & 6 & $\mathbf{4 0}$ & 26.4 & 10.5 & 3.2 & $\mathbf{4 0}$ \\
\hline Total & $\mathbf{5 8}$ & $\mathbf{2 3}$ & $\mathbf{7}$ & $\mathbf{8 8}$ & $\mathbf{5 8}$ & $\mathbf{2 3}$ & $\mathbf{7}$ & $\mathbf{8 8}$ \\
\hline
\end{tabular}

Chi-Square Value: 5.08 Degrees of Freedom: 2

Critical Value: 5.99

Table 60: Chi-Square Results 6 Cluster Solution Level III, Clusters 6-4 and 6-5

\begin{tabular}{|c|c|c|c|c|c|c|c|c|}
\hline Level III & \multicolumn{3}{|c|}{ Observed Frequency } & Total & \multicolumn{3}{c|}{ Expected Frequency } & Total \\
\hline & Bone & $\begin{array}{c}\text { Chips and } \\
\text { Flakes }\end{array}$ & $\begin{array}{c}\text { Stone } \\
\text { Tools }\end{array}$ & & Bone & $\begin{array}{c}\text { Chips and } \\
\text { Flakes }\end{array}$ & $\begin{array}{c}\text { Stone } \\
\text { Tools }\end{array}$ & \\
\hline Cluster 6-4 & 33 & 14 & 1 & $\mathbf{4 8}$ & 25.4 & 20.5 & 2.1 & $\mathbf{4 8}$ \\
\hline Cluster 6-6 & 3 & 15 & 2 & $\mathbf{2 0}$ & 10.6 & 8.5 & 0.9 & $\mathbf{2 0}$ \\
\hline Total & $\mathbf{3 6}$ & $\mathbf{2 9}$ & $\mathbf{3}$ & $\mathbf{6 8}$ & $\mathbf{3 6}$ & $\mathbf{2 9}$ & $\mathbf{3}$ & $\mathbf{6 8}$ \\
\hline
\end{tabular}

Chi-Square Value: 16.66 Degrees of Freedom: 2

Critical Value: 5.99

Table 61: Chi-Square Results 6 Cluster Solution Level III, Clusters 6-4 and 6-6

\begin{tabular}{|c|c|c|c|c|c|c|c|c|}
\hline Level III & \multicolumn{3}{|c|}{ Observed Frequency } & Total & \multicolumn{3}{|c|}{ Expected Frequency } & Total \\
\hline & Bone & $\begin{array}{l}\text { Chips and } \\
\text { Flakes } \\
\end{array}$ & $\begin{array}{l}\text { Stone } \\
\text { Tools }\end{array}$ & & Bone & $\begin{array}{l}\text { Chips and } \\
\text { Flakes }\end{array}$ & $\begin{array}{l}\text { Stone } \\
\text { Tools }\end{array}$ & \\
\hline Cluster 6-5 & 25 & 9 & 6 & 40 & 18.7 & 16.0 & 5.3 & 40 \\
\hline Cluster 6-6 & 3 & 15 & 2 & 20 & 9.3 & 8.0 & 2.7 & 20 \\
\hline Total & 28 & 24 & 8 & 60 & 28 & 24 & 8 & 60 \\
\hline
\end{tabular}

Chi-Square Value: 15.88 Degrees of Freedom: 2

Critical Value: 5.99

Table 62: Chi-Square Results 6 Cluster Solution Level III, Clusters 6-5 and 6-6 


\section{Chi-Square Contingency Table Results - 3 Cluster Solution Level IV}

\begin{tabular}{|c|c|c|c|c|c|c|c|c|}
\hline Level IV & \multicolumn{3}{|c|}{ Observed Frequency } & Total & \multicolumn{3}{c|}{ Expected Frequency } & Total \\
\hline & Bone & $\begin{array}{c}\text { Chips and } \\
\text { Flakes }\end{array}$ & $\begin{array}{c}\text { Stone } \\
\text { Tools }\end{array}$ & & Bone & $\begin{array}{c}\text { Chips and } \\
\text { Flakes }\end{array}$ & $\begin{array}{c}\text { Stone } \\
\text { Tools }\end{array}$ & \\
\hline Cluster 3-1 & 36 & 39 & 9 & $\mathbf{8 4}$ & 37.2 & 36.3 & 10.5 & $\mathbf{8 4}$ \\
\hline Cluster 3-2 & 42 & 37 & 13 & $\mathbf{9 2}$ & 40.8 & 39.7 & 11.5 & $\mathbf{9 2}$ \\
\hline Total & $\mathbf{7 8}$ & $\mathbf{7 6}$ & $\mathbf{2 2}$ & $\mathbf{1 7 6}$ & $\mathbf{7 8}$ & $\mathbf{7 6}$ & $\mathbf{2 2}$ & $\mathbf{1 7 6}$ \\
\hline
\end{tabular}

Chi-Square Value: 0.88 Degrees of Freedom: 2

Critical Value: 5.99

Table 63: Chi-Square Results 3 Cluster Solution Level IV, Clusters 3-1 and 3-2

\begin{tabular}{|c|c|c|c|c|c|c|c|c|}
\hline Level IV & \multicolumn{3}{|c|}{ Observed Frequency } & Total & \multicolumn{3}{|c|}{ Expected Frequency } & Total \\
\hline & Bone & $\begin{array}{c}\text { Chips and } \\
\text { Flakes }\end{array}$ & $\begin{array}{l}\text { Stone } \\
\text { Tools }\end{array}$ & & Bone & $\begin{array}{c}\text { Chips and } \\
\text { Flakes }\end{array}$ & $\begin{array}{l}\text { Stone } \\
\text { Tools }\end{array}$ & \\
\hline Cluster 3-1 & 36 & 39 & 9 & 84 & 40.6 & 33.7 & 9.7 & 84 \\
\hline Cluster 3-3 & 35 & 20 & 8 & 63 & 30.4 & 25.3 & 7.3 & 63 \\
\hline Total & 71 & 59 & 17 & 147 & 71 & 59 & 17 & 147 \\
\hline
\end{tabular}

Chi-Square Value: 3.26 Degrees of Freedom: 2

Critical Value: 5.99

Table 64: Chi-Square Results 3 Cluster Solution Level IV, Clusters 3-1 and 3-3

\begin{tabular}{|c|c|c|c|c|c|c|c|c|}
\hline Level IV & \multicolumn{3}{|c|}{ Observed Frequency } & Total & \multicolumn{3}{c|}{ Expected Frequency } & Total \\
\hline & Bone & $\begin{array}{c}\text { Chips and } \\
\text { Flakes }\end{array}$ & $\begin{array}{c}\text { Stone } \\
\text { Tools }\end{array}$ & & Bone & $\begin{array}{c}\text { Chips and } \\
\text { Flakes }\end{array}$ & $\begin{array}{c}\text { Stone } \\
\text { Tools }\end{array}$ & \\
\hline Cluster 3-2 & 42 & 37 & 13 & $\mathbf{9 2}$ & 45.7 & 33.8 & 12.5 & $\mathbf{9 2}$ \\
\hline Cluster 3-3 & 35 & 20 & $\mathbf{8}$ & $\mathbf{6 3}$ & 31.3 & 23.2 & 8.5 & $\mathbf{6 3}$ \\
\hline Total & $\mathbf{7 7}$ & $\mathbf{5 7}$ & $\mathbf{2 1}$ & $\mathbf{1 5 5}$ & $\mathbf{7 7}$ & $\mathbf{5 7}$ & $\mathbf{2 1}$ & $\mathbf{1 5 5}$ \\
\hline
\end{tabular}

Chi-Square Value: 1.52 Degrees of Freedom: 2

Critical Value: 5.99

Table 65: Chi-Square Results 3 Cluster Solution Level IV, Clusters 3-2 and 3-3 


\section{Chi-Square Contingency Table Results - 5 Cluster Solution Level IV}

\begin{tabular}{|c|c|c|c|c|c|c|c|c|}
\hline Level IV & \multicolumn{3}{|c|}{ Observed Frequency } & Total & \multicolumn{3}{c|}{ Expected Frequency } & Total \\
\hline & Bone & $\begin{array}{c}\text { Chips and } \\
\text { Flakes }\end{array}$ & $\begin{array}{c}\text { Stone } \\
\text { Tools }\end{array}$ & & Bone & $\begin{array}{c}\text { Chips and } \\
\text { Flakes }\end{array}$ & $\begin{array}{c}\text { Stone } \\
\text { Tools }\end{array}$ & \\
\hline Cluster 5-1 & 19 & 22 & 1 & $\mathbf{4 2}$ & 18.7 & 19.9 & 3.4 & $\mathbf{4 2}$ \\
\hline Cluster 5-2 & 14 & 13 & 5 & $\mathbf{3 2}$ & 14.3 & 15.1 & 2.6 & $\mathbf{3 2}$ \\
\hline Total & $\mathbf{3 3}$ & $\mathbf{3 5}$ & $\mathbf{6}$ & $\mathbf{7 4}$ & $\mathbf{3 3}$ & $\mathbf{3 5}$ & $\mathbf{6}$ & $\mathbf{7 4}$ \\
\hline
\end{tabular}

Chi-Square Value: 4.47 Degrees of Freedom: 2

Critical Value: 5.99

Table 66: Chi-Square Results 5 Cluster Solution Level IV, Clusters 5-1 and 5-2

\begin{tabular}{|c|c|c|c|c|c|c|c|c|}
\hline Level IV & \multicolumn{3}{|c|}{ Observed Frequency } & Total & \multicolumn{3}{c|}{ Expected Frequency } & Total \\
\hline & Bone & $\begin{array}{c}\text { Chips and } \\
\text { Flakes }\end{array}$ & $\begin{array}{c}\text { Stone } \\
\text { Tools }\end{array}$ & & Bone & $\begin{array}{c}\text { Chips and } \\
\text { Flakes }\end{array}$ & $\begin{array}{c}\text { Stone } \\
\text { Tools }\end{array}$ & \\
\hline Cluster 5-1 & 19 & 22 & 1 & $\mathbf{4 2}$ & 17.6 & 20.2 & 4.2 & $\mathbf{4 2}$ \\
\hline Cluster 5-3 & 23 & 26 & 9 & $\mathbf{5 8}$ & 24.4 & 27.8 & 5.8 & $\mathbf{5 8}$ \\
\hline Total & $\mathbf{4 2}$ & $\mathbf{4 8}$ & $\mathbf{1 0}$ & $\mathbf{1 0 0}$ & $\mathbf{4 2}$ & $\mathbf{4 8}$ & $\mathbf{1 0}$ & $\mathbf{1 0 0}$ \\
\hline
\end{tabular}

Chi-Square Value: 4.67 Degrees of Freedom: 2

Critical Value: 5.99

Table 67: Chi-Square Results 5 Cluster Solution Level IV, Clusters 5-1 and 5-3

\begin{tabular}{|c|c|c|c|c|c|c|c|c|}
\hline Level IV & \multicolumn{3}{|c|}{ Observed Frequency } & Total & \multicolumn{3}{|c|}{ Expected Frequency } & Total \\
\hline & Bone & $\begin{array}{c}\text { Chips and } \\
\text { Flakes }\end{array}$ & $\begin{array}{c}\text { Stone } \\
\text { Tools }\end{array}$ & & Bone & $\begin{array}{c}\text { Chips and } \\
\text { Flakes }\end{array}$ & $\begin{array}{c}\text { Stone } \\
\text { Tools }\end{array}$ & \\
\hline Cluster 5-1 & 19 & 22 & 1 & $\mathbf{4 2}$ & 20.7 & 17.5 & 3.8 & $\mathbf{4 2}$ \\
\hline Cluster 5-4 & 19 & 10 & 6 & $\mathbf{3 5}$ & 17.3 & 14.5 & 3.2 & $\mathbf{3 5}$ \\
\hline Total & $\mathbf{3 8}$ & $\mathbf{3 2}$ & $\mathbf{7}$ & $\mathbf{7 7}$ & $\mathbf{3 8}$ & $\mathbf{3 2}$ & $\mathbf{7}$ & $\mathbf{7 7}$ \\
\hline
\end{tabular}

Chi-Square Value: $7.50 \quad$ Degrees of Freedom: 2

Critical Value: 5.99

Table 68: Chi-Square Results 5 Cluster Solution Level IV, Clusters 5-1 and 5-4 


\begin{tabular}{|c|c|c|c|c|c|c|c|c|}
\hline Level IV & \multicolumn{3}{|c|}{ Observed Frequency } & Total & \multicolumn{3}{c|}{ Expected Frequency } & Total \\
\hline & Bone & $\begin{array}{c}\text { Chips and } \\
\text { Flakes }\end{array}$ & $\begin{array}{c}\text { Stone } \\
\text { Tools }\end{array}$ & & Bone & $\begin{array}{c}\text { Chips and } \\
\text { Flakes }\end{array}$ & $\begin{array}{c}\text { Stone } \\
\text { Tools }\end{array}$ & \\
\hline Cluster 5-1 & 19 & 22 & 1 & $\mathbf{4 2}$ & 21.0 & 17.3 & 3.7 & $\mathbf{4 2}$ \\
\hline Cluster 5-5 & 38 & 25 & 9 & $\mathbf{7 2}$ & 36.0 & 29.7 & 6.3 & $\mathbf{7 2}$ \\
\hline Total & $\mathbf{5 7}$ & $\mathbf{4 7}$ & $\mathbf{1 0}$ & $\mathbf{1 1 4}$ & $\mathbf{5 7}$ & $\mathbf{4 7}$ & $\mathbf{1 0}$ & $\mathbf{1 1 4}$ \\
\hline
\end{tabular}

Chi-Square Value: 5.40 Degrees of Freedom: 2

Critical Value: 5.99

Table 69: Chi-Square Results 5 Cluster Solution Level IV, Clusters 5-1 and 5-5

\begin{tabular}{|c|c|c|c|c|c|c|c|c|}
\hline Level IV & \multicolumn{3}{|c|}{ Observed Frequency } & Total & \multicolumn{3}{c|}{ Expected Frequency } & Total \\
\hline & Bone & $\begin{array}{c}\text { Chips and } \\
\text { Flakes }\end{array}$ & $\begin{array}{c}\text { Stone } \\
\text { Tools }\end{array}$ & & Bone & $\begin{array}{c}\text { Chips and } \\
\text { Flakes }\end{array}$ & $\begin{array}{c}\text { Stone } \\
\text { Tools }\end{array}$ & \\
\hline Cluster 5-2 & 14 & 13 & 5 & $\mathbf{3 2}$ & 13.2 & 13.9 & 5.0 & $\mathbf{3 2}$ \\
\hline Cluster 5-3 & 23 & 26 & 9 & $\mathbf{5 8}$ & 23.8 & 25.1 & 9.0 & $\mathbf{5 8}$ \\
\hline Total & $\mathbf{3 7}$ & $\mathbf{3 9}$ & $\mathbf{1 4}$ & $\mathbf{9 0}$ & $\mathbf{3 7}$ & $\mathbf{3 9}$ & $\mathbf{1 4}$ & $\mathbf{9 0}$ \\
\hline
\end{tabular}

Chi-Square Value: 0.17 Degrees of Freedom: 2

Critical Value: 5.99

Table 70: Chi-Square Results 5 Cluster Solution Level IV, Clusters 5-2 and 5-3

\begin{tabular}{|c|c|c|c|c|c|c|c|c|}
\hline Level IV & \multicolumn{3}{|c|}{ Observed Frequency } & Total & \multicolumn{3}{|c|}{ Expected Frequency } & Total \\
\hline & Bone & $\begin{array}{c}\text { Chips and } \\
\text { Flakes }\end{array}$ & $\begin{array}{c}\text { Stone } \\
\text { Tools }\end{array}$ & & Bone & $\begin{array}{c}\text { Chips and } \\
\text { Flakes }\end{array}$ & $\begin{array}{c}\text { Stone } \\
\text { Tools }\end{array}$ & \\
\hline Cluster 5-2 & 14 & 13 & 5 & $\mathbf{3 2}$ & 15.8 & 11.0 & 5.3 & $\mathbf{3 2}$ \\
\hline Cluster 5-4 & 19 & 10 & 6 & $\mathbf{3 5}$ & 17.2 & 12.0 & 5.7 & $\mathbf{3 5}$ \\
\hline Total & $\mathbf{3 3}$ & $\mathbf{2 3}$ & $\mathbf{1 1}$ & $\mathbf{6 7}$ & $\mathbf{3 3}$ & $\mathbf{2 3}$ & $\mathbf{1 1}$ & $\mathbf{6 7}$ \\
\hline
\end{tabular}

Chi-Square Value: $1.11 \quad$ Degrees of Freedom: 2

Critical Value: 5.99

Table 71: Chi-Square Results 5 Cluster Solution Level IV, Clusters 5-2 and 5-4

\begin{tabular}{|c|c|c|c|c|c|c|c|c|}
\hline Level IV & \multicolumn{3}{|c|}{ Observed Frequency } & Total & \multicolumn{3}{c|}{ Expected Frequency } & Total \\
\hline & Bone & $\begin{array}{c}\text { Chips and } \\
\text { Flakes }\end{array}$ & $\begin{array}{c}\text { Stone } \\
\text { Tools }\end{array}$ & & Bone & $\begin{array}{c}\text { Chips and } \\
\text { Flakes }\end{array}$ & $\begin{array}{c}\text { Stone } \\
\text { Tools }\end{array}$ & \\
\hline Cluster 5-2 & $\mathbf{1 4}$ & 13 & 5 & $\mathbf{3 2}$ & 16.0 & 11.7 & 4.3 & $\mathbf{3 2}$ \\
\hline Cluster 5-5 & 38 & 25 & 9 & $\mathbf{7 2}$ & 36.0 & 26.3 & 9.7 & $\mathbf{7 2}$ \\
\hline Total & $\mathbf{5 2}$ & $\mathbf{3 8}$ & $\mathbf{1 4}$ & $\mathbf{1 0 4}$ & $\mathbf{5 2}$ & $\mathbf{3 8}$ & $\mathbf{1 4}$ & $\mathbf{1 0 4}$ \\
\hline
\end{tabular}

Chi-Square Value: 0.73 Degrees of Freedom: 2

Critical Value: 5.99

Table 72: Chi-Square Results 5 Cluster Solution Level IV, Clusters 5-2 and 5-5 


\begin{tabular}{|c|c|c|c|c|c|c|c|c|}
\hline Level IV & \multicolumn{3}{|c|}{ Observed Frequency } & Total & \multicolumn{3}{c|}{ Expected Frequency } & Total \\
\hline & Bone & $\begin{array}{c}\text { Chips and } \\
\text { Flakes }\end{array}$ & $\begin{array}{c}\text { Stone } \\
\text { Tools }\end{array}$ & & Bone & $\begin{array}{c}\text { Chips and } \\
\text { Flakes }\end{array}$ & $\begin{array}{c}\text { Stone } \\
\text { Tools }\end{array}$ & \\
\hline Cluster 5-3 & 23 & 26 & 9 & $\mathbf{5 8}$ & 26.2 & 22.5 & 9.4 & $\mathbf{5 8}$ \\
\hline Cluster 5-4 & 19 & 10 & 6 & $\mathbf{3 5}$ & 15.8 & 13.5 & 5.6 & $\mathbf{3 5}$ \\
\hline Total & $\mathbf{4 2}$ & $\mathbf{3 6}$ & $\mathbf{1 5}$ & $\mathbf{9 3}$ & $\mathbf{4 2}$ & $\mathbf{3 6}$ & $\mathbf{1 5}$ & $\mathbf{9 3}$ \\
\hline
\end{tabular}

Chi-Square Value: 2.56 Degrees of Freedom: 2

Critical Value: 5.99

Table 73: Chi-Square Results 5 Cluster Solution Level IV, Clusters 5-3 and 5-4

\begin{tabular}{|c|c|c|c|c|c|c|c|c|}
\hline Level IV & \multicolumn{3}{|c|}{ Observed Frequency } & Total & \multicolumn{3}{c|}{ Expected Frequency } & Total \\
\hline & Bone & $\begin{array}{c}\text { Chips and } \\
\text { Flakes }\end{array}$ & $\begin{array}{c}\text { Stone } \\
\text { Tools }\end{array}$ & & Bone & $\begin{array}{c}\text { Chips and } \\
\text { Flakes }\end{array}$ & $\begin{array}{c}\text { Stone } \\
\text { Tools }\end{array}$ & \\
\hline Cluster 5-3 & 23 & 26 & 9 & $\mathbf{5 8}$ & 27.2 & 22.8 & 8.0 & $\mathbf{5 8}$ \\
\hline Cluster 5-5 & 38 & 25 & 9 & $\mathbf{7 2}$ & 33.8 & 28.2 & 10.0 & $\mathbf{7 2}$ \\
\hline Total & $\mathbf{6 1}$ & $\mathbf{5 1}$ & $\mathbf{1 8}$ & $\mathbf{1 3 0}$ & $\mathbf{6 1}$ & $\mathbf{5 1}$ & $\mathbf{1 8}$ & $\mathbf{1 3 0}$ \\
\hline
\end{tabular}

Chi-Square Value: 2.23 Degrees of Freedom: 2

Critical Value: 5.99

Table 74: Chi-Square Results 5 Cluster Solution Level IV, Clusters 5-3 and 5-5

\begin{tabular}{|c|c|c|c|c|c|c|c|c|}
\hline Level IV & \multicolumn{3}{|c|}{ Observed Frequency } & Total & \multicolumn{3}{c|}{ Expected Frequency } & Total \\
\hline & Bone & $\begin{array}{c}\text { Chips and } \\
\text { Flakes }\end{array}$ & $\begin{array}{c}\text { Stone } \\
\text { Tools }\end{array}$ & & Bone & $\begin{array}{c}\text { Chips and } \\
\text { Flakes }\end{array}$ & $\begin{array}{c}\text { Stone } \\
\text { Tools }\end{array}$ & \\
\hline Cluster 5-4 & 19 & 10 & 6 & $\mathbf{3 5}$ & 18.6 & 11.4 & 4.9 & $\mathbf{3 5}$ \\
\hline Cluster 5-5 & 38 & 25 & 9 & $\mathbf{7 2}$ & 38.4 & 23.6 & 10.1 & $\mathbf{7 2}$ \\
\hline Total & $\mathbf{5 7}$ & $\mathbf{3 5}$ & $\mathbf{1 5}$ & $\mathbf{1 0 7}$ & $\mathbf{5 7}$ & $\mathbf{3 5}$ & $\mathbf{1 5}$ & $\mathbf{1 0 7}$ \\
\hline
\end{tabular}

Chi-Square Value: 0.64 Degrees of Freedom: 2

Critical Value: 5.99

Table 75: Chi-Square Results 5 Cluster Solution Level IV, Clusters 5-4 and 5-5 
- Appendix E -

Horizontal K-Means Cluster Distributions Re-Sampled Levels 


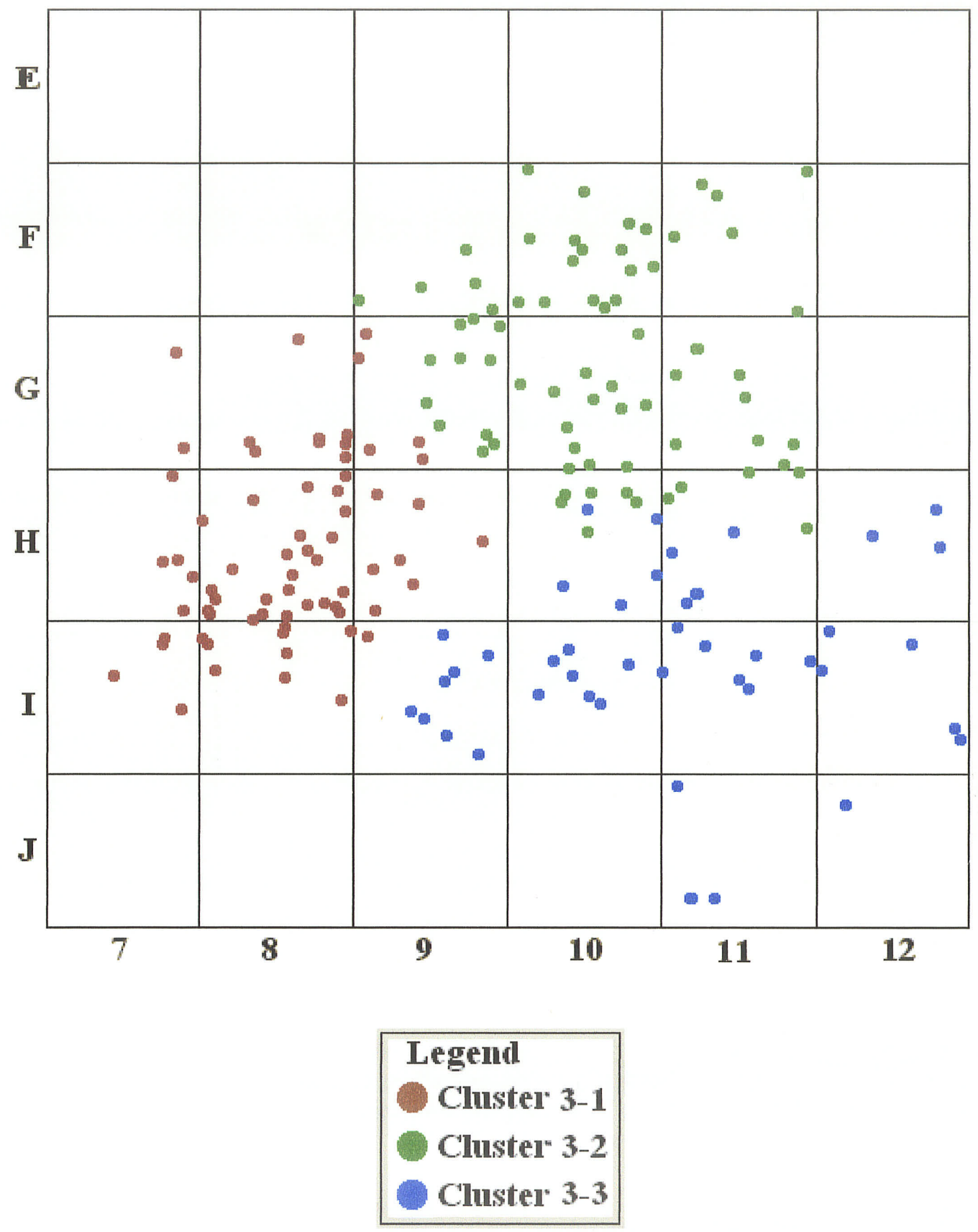

Figure 1: Horizontal Distribution 3 Cluster Solution, Level II/2 


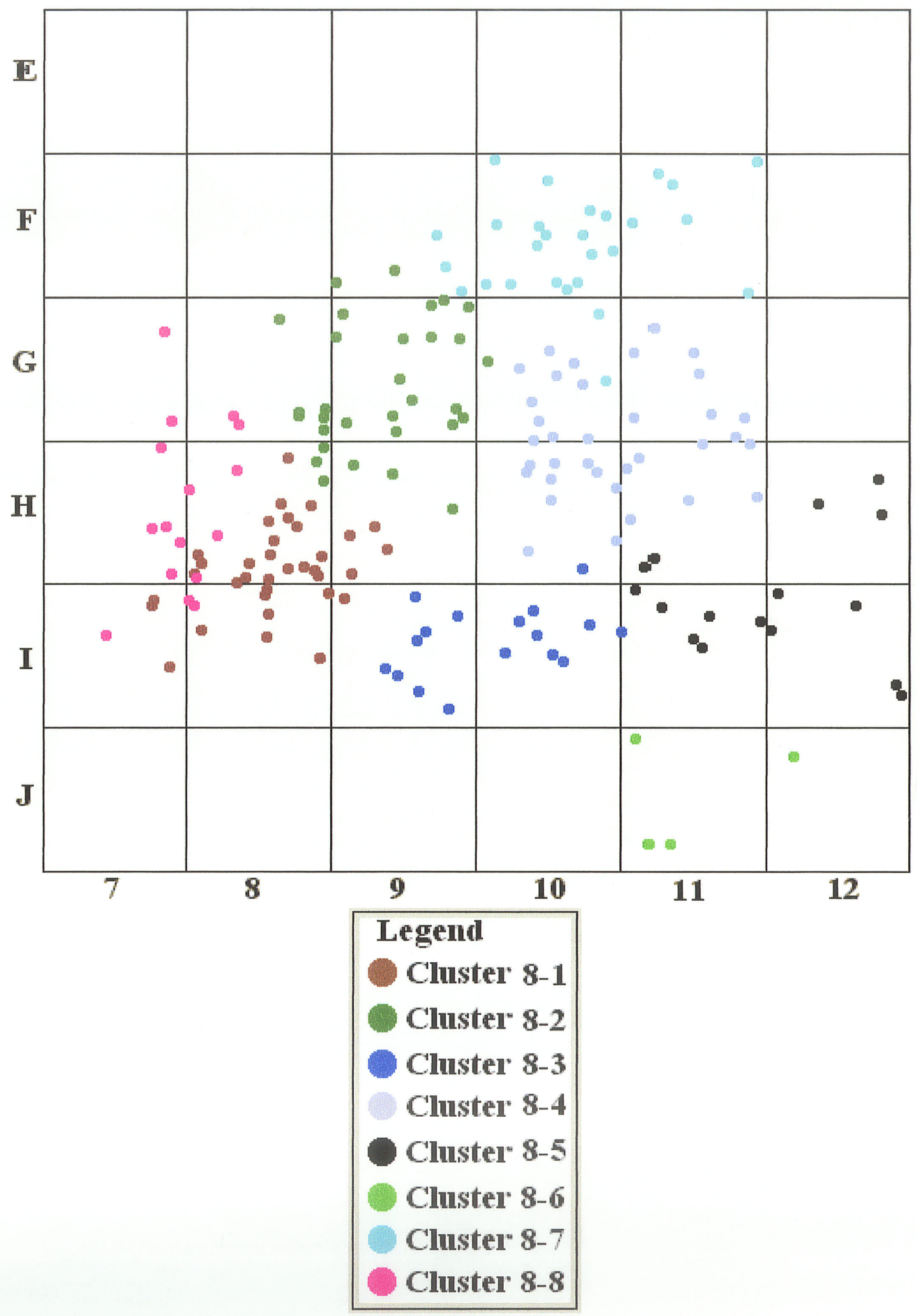

Figure 2: Horizontal Distribution 8 Cluster Solution, Level II/2 


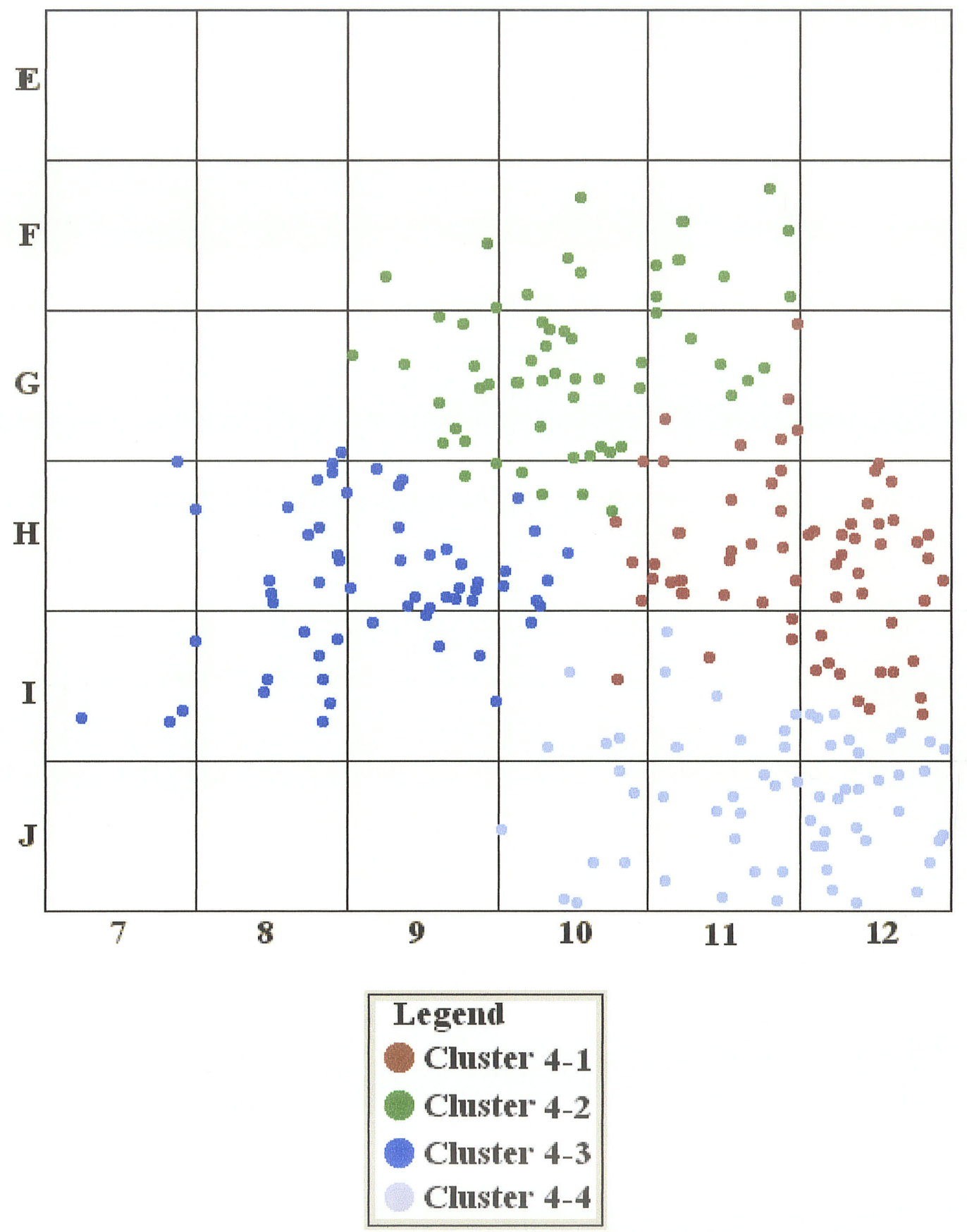

Figure 3: Horizontal Distribution 4 Cluster Solution, Level III 


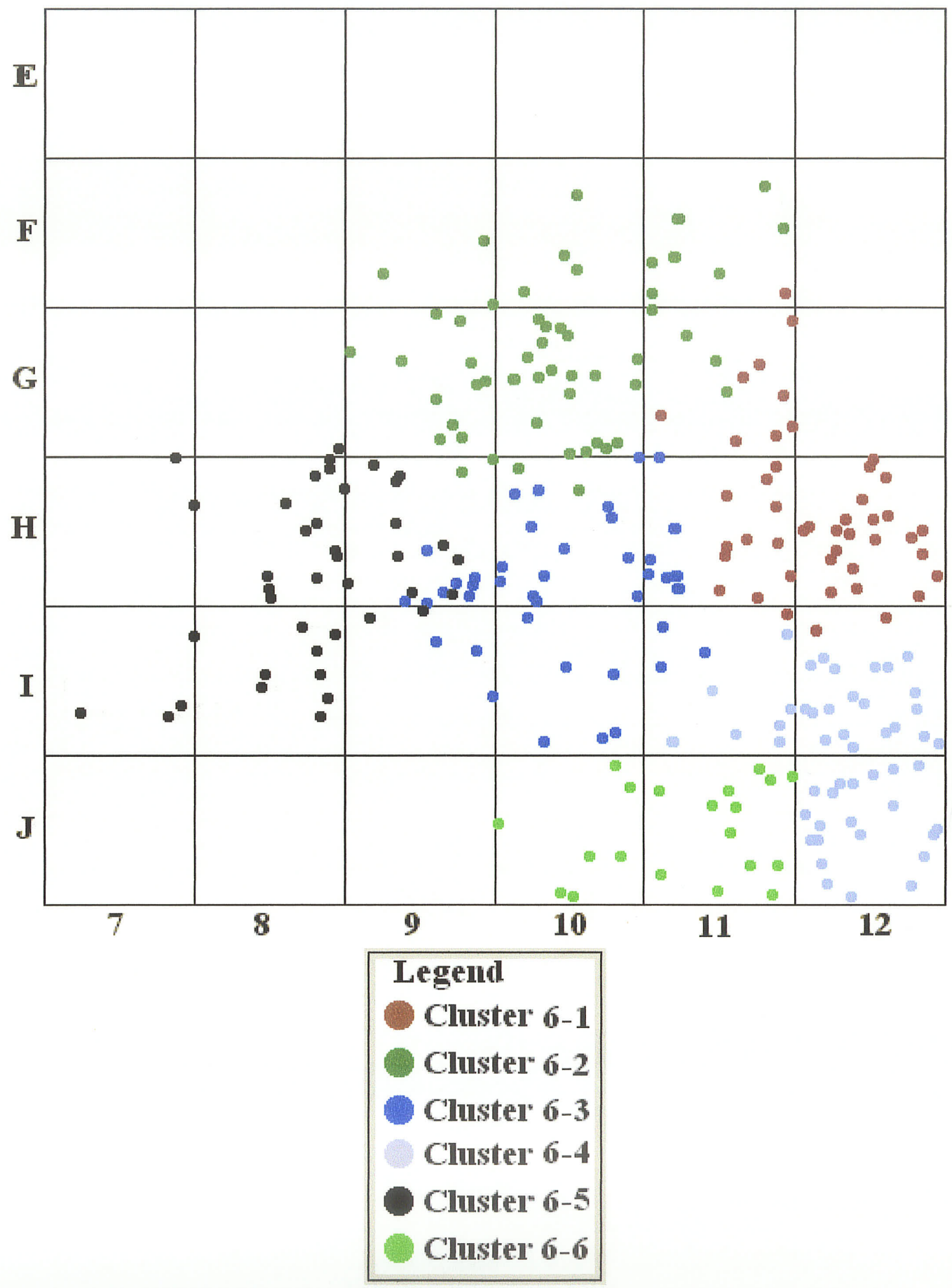

Figure 4: Horizontal Distribution 6 Cluster Solution, Level III 


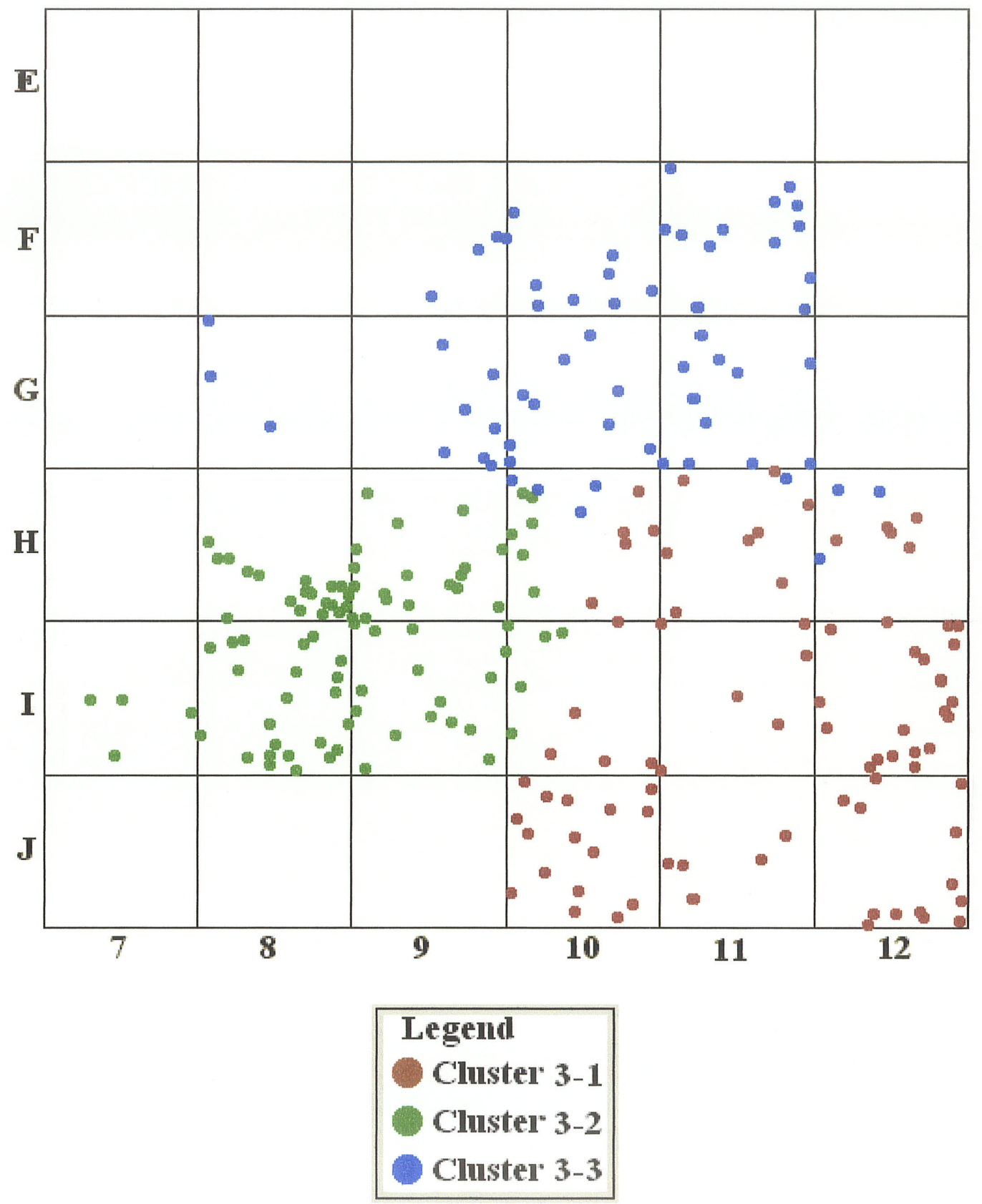

Figure 5: Horizontal Distribution 3 Cluster Solution, Level IV 


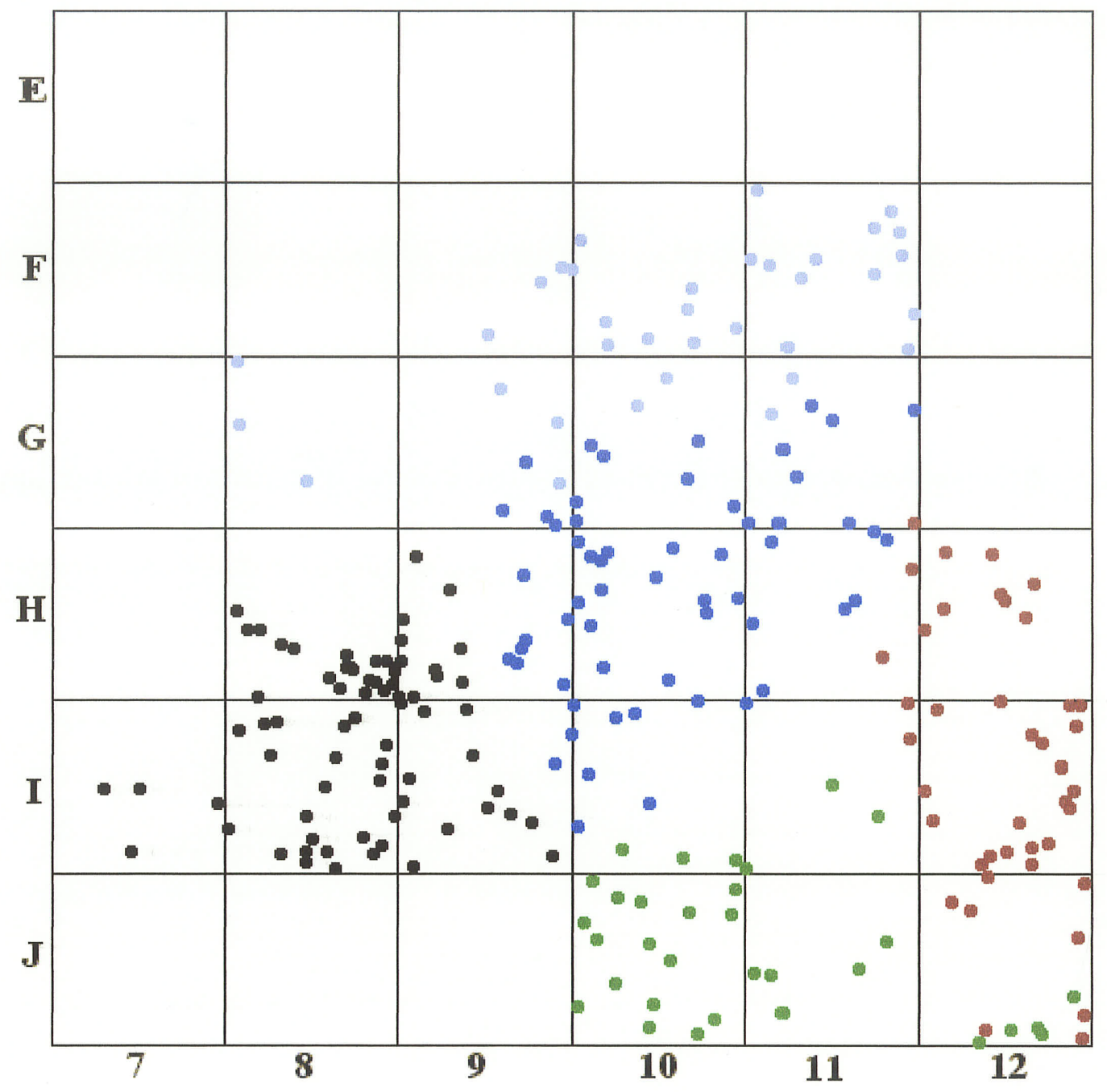

\begin{tabular}{|l|}
\hline Legend \\
Cluster" 5-1 \\
Cluster" 5-2 \\
Cluster" 5-3 \\
Cluster" 5-4 \\
Cluster" 5-5
\end{tabular}

Figure 6: Horizontal Distribution 5 Cluster Solution, Level IV 
- Appendix F -

Three-Dimensional K-Means Cluster Distributions Re-Sampled Levels 


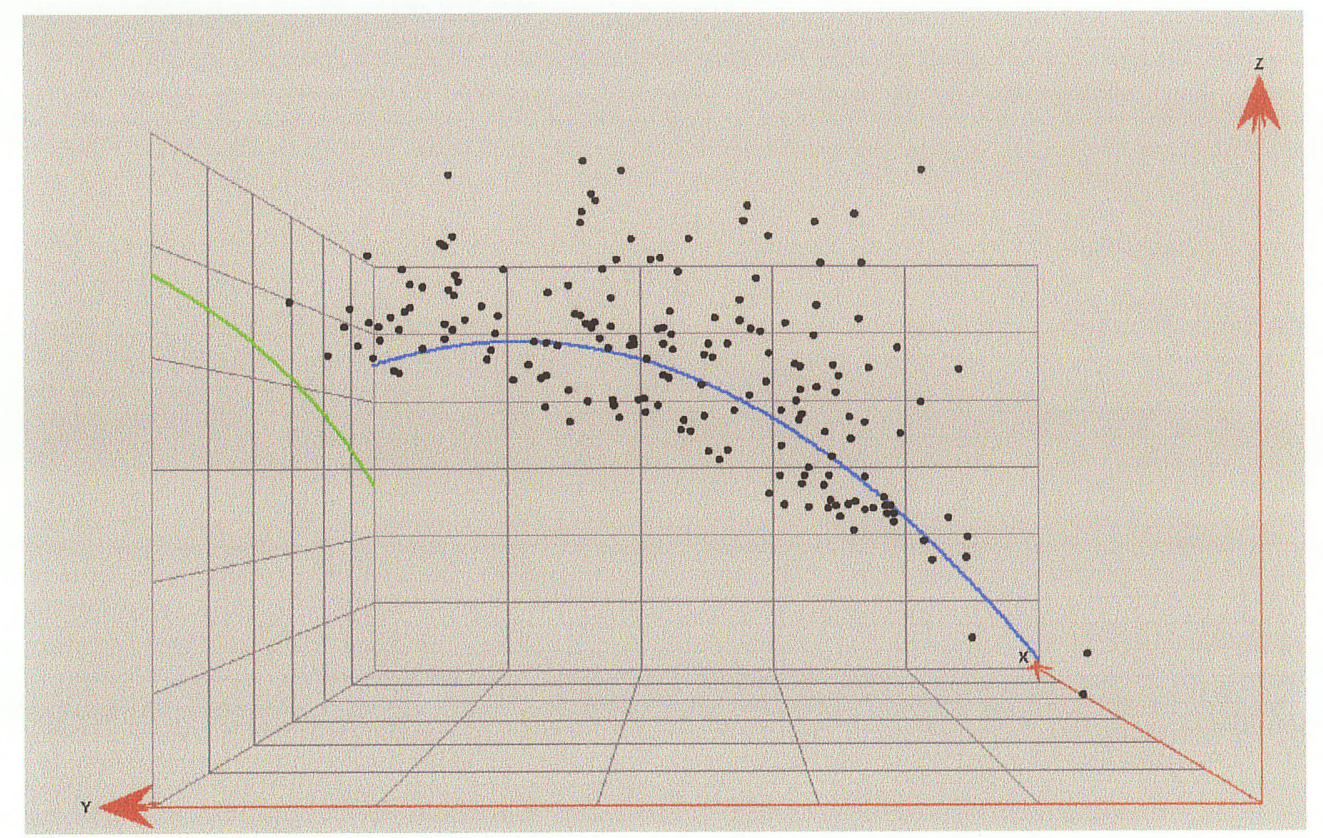

Figure 1: Three-Dimensional Distribution - Level II/2

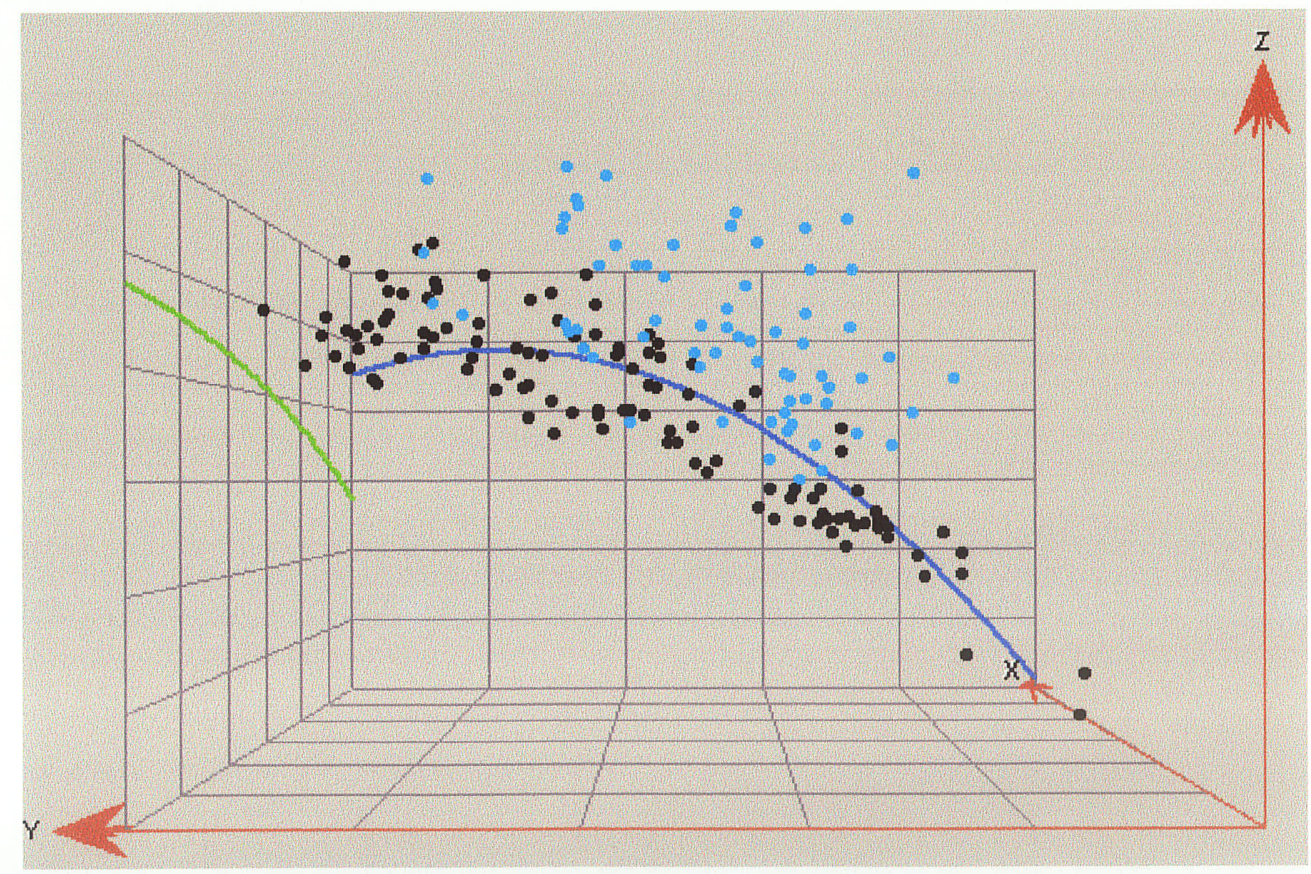

Figure 2: Three-Dimensional Plot - Three Cluster Solution: Cluster 3-1, Level III/2 


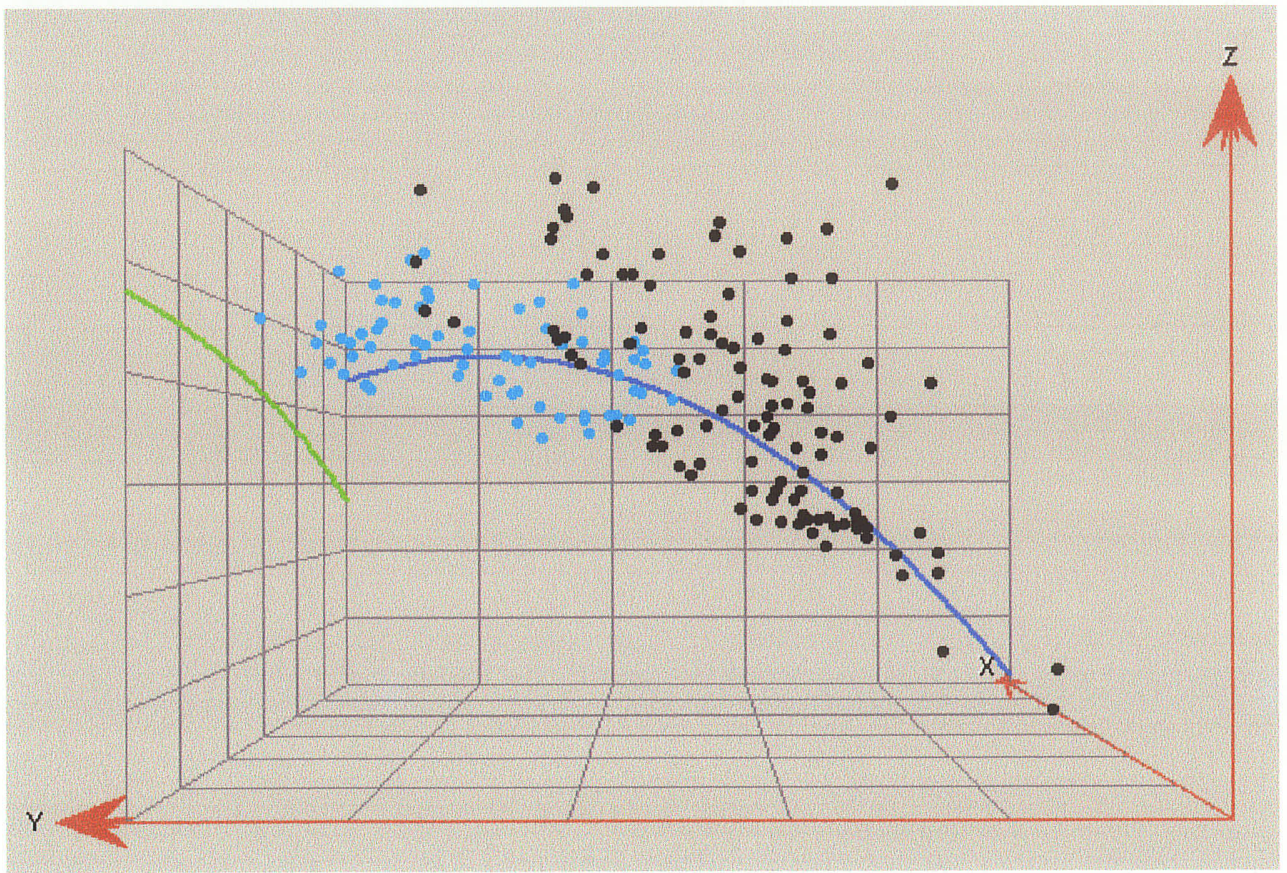

Figure 3: Three-Dimensional Plot - Three Cluster Solution: Cluster 3-2, Level II/2

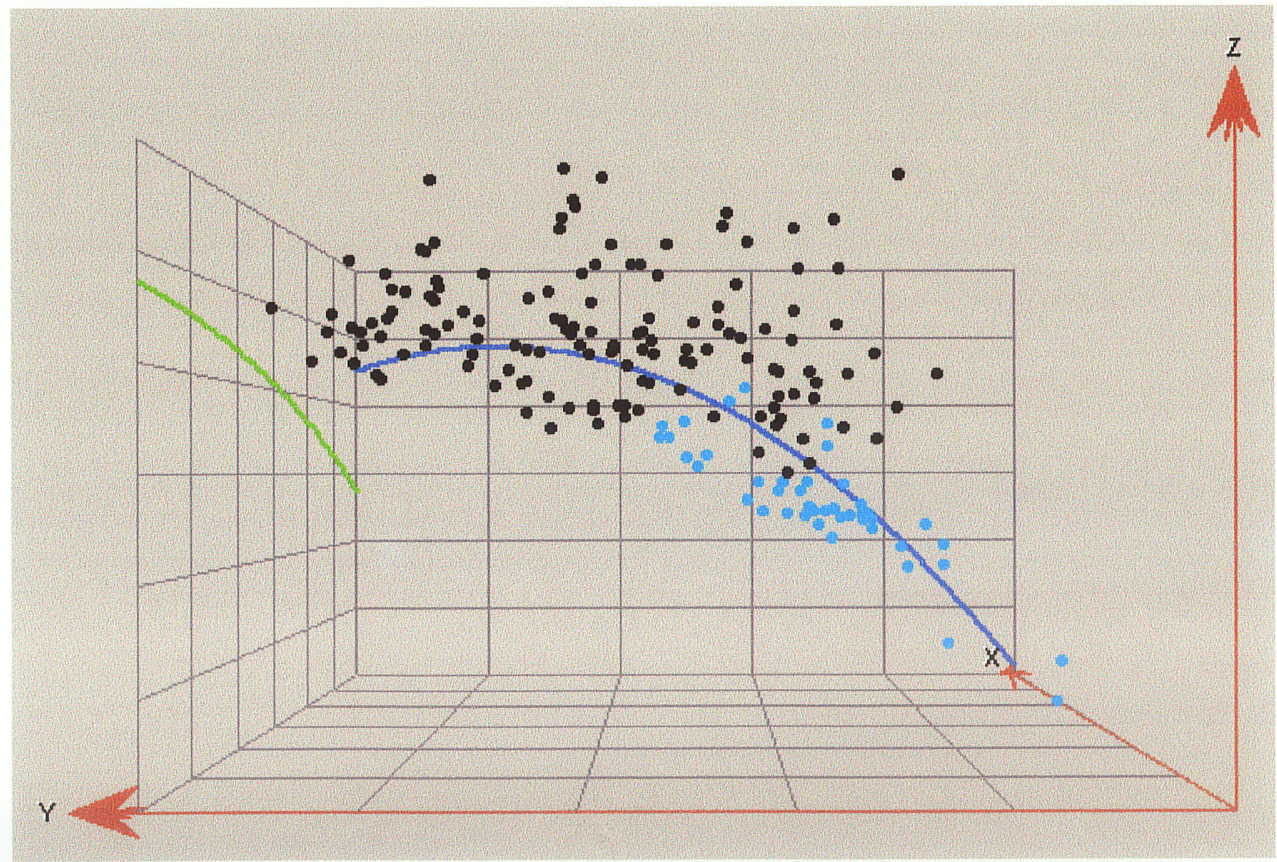

Figure 4: Three-Dimensional Plot - Three Cluster Solution: Cluster 3-3, Level II/2 


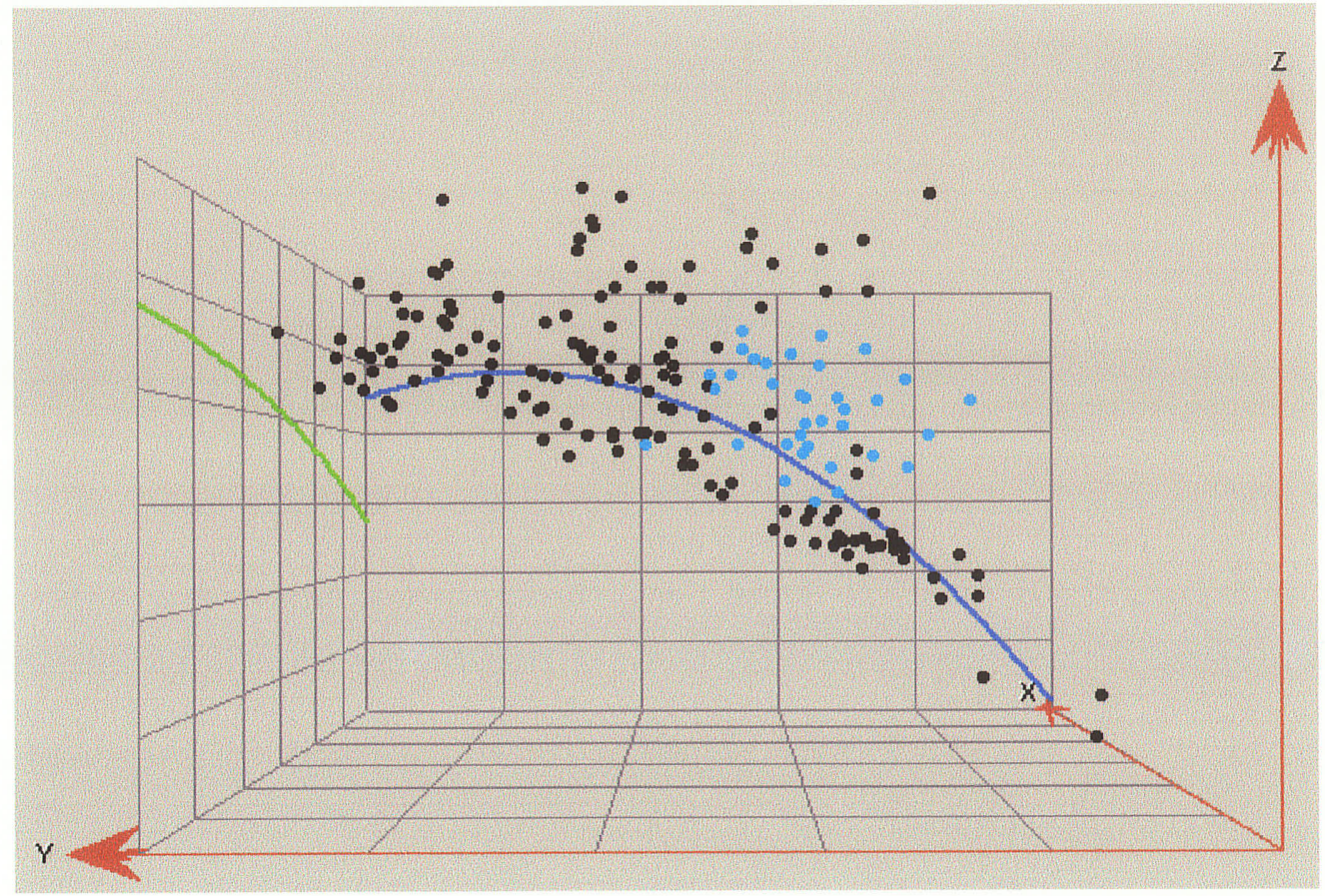

Figure 5: Three-Dimensional Plot - Eight Cluster Solution: Cluster 8-1, Level II/2

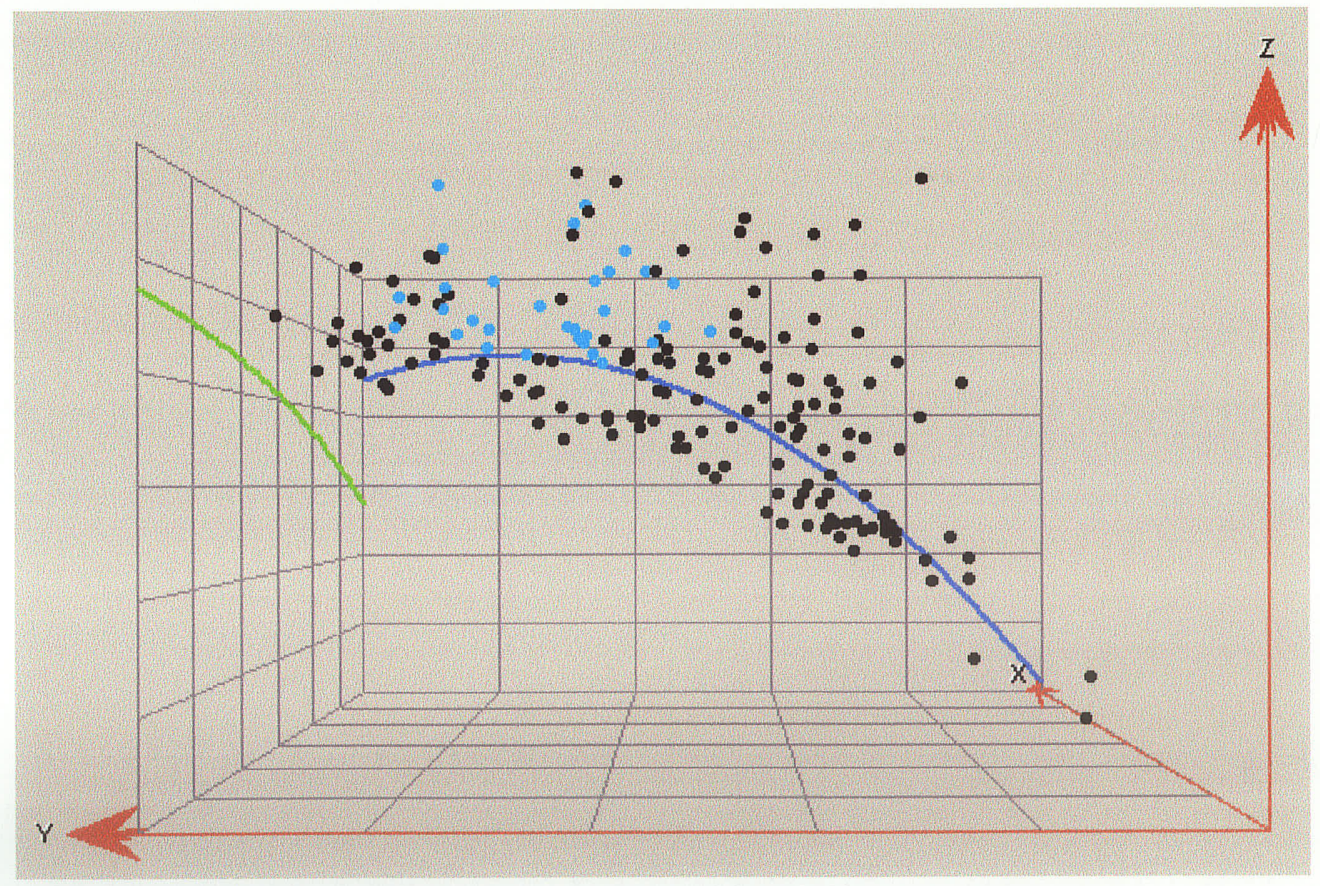

Figure 6: Three-Dimensional Plot - Eight Cluster Solution: Cluster 8-2, Level II/2 


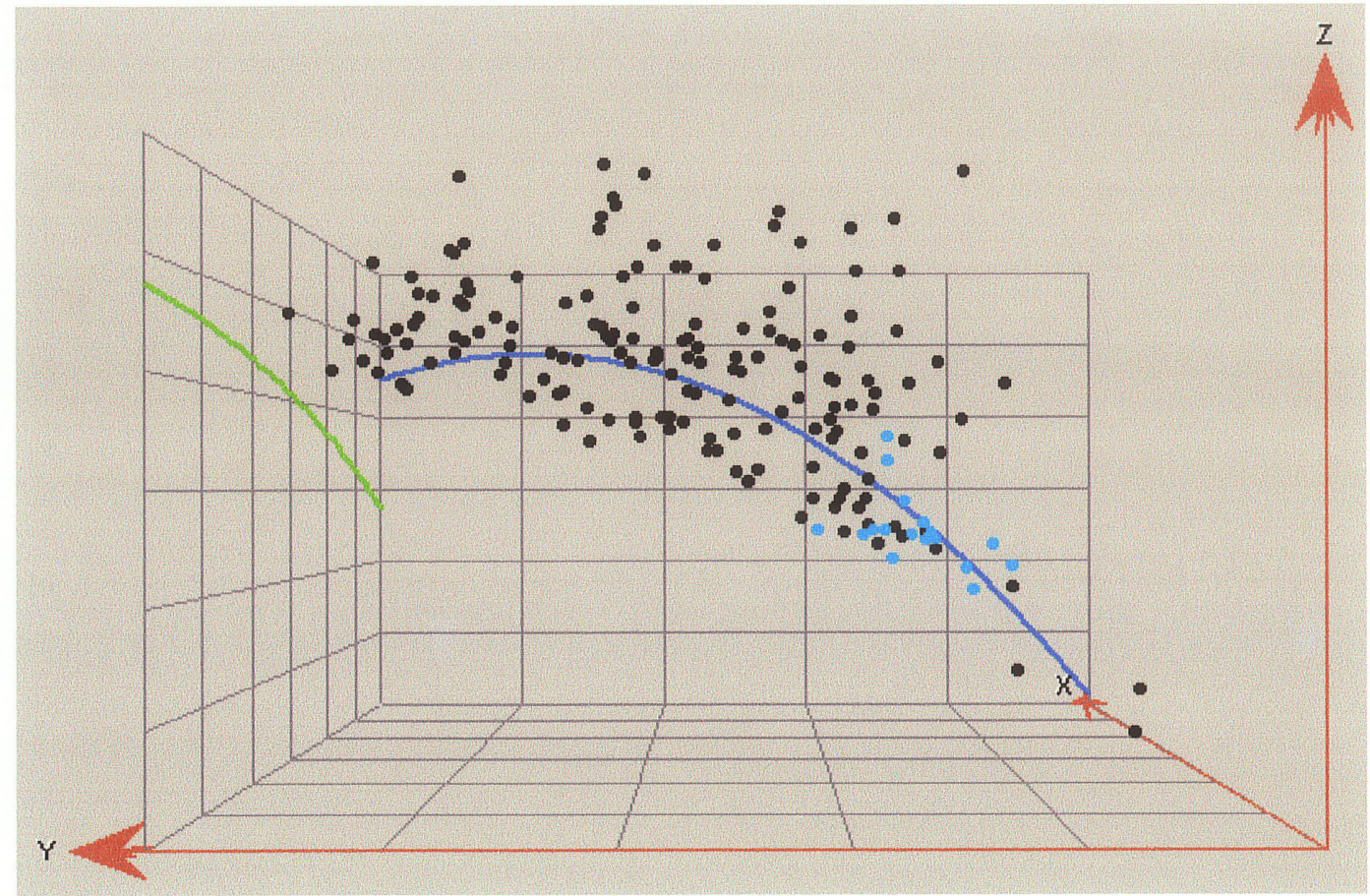

Figure 7: Three-Dimensional Plot - Eight Cluster Solution: Cluster 8-3, Level II/2

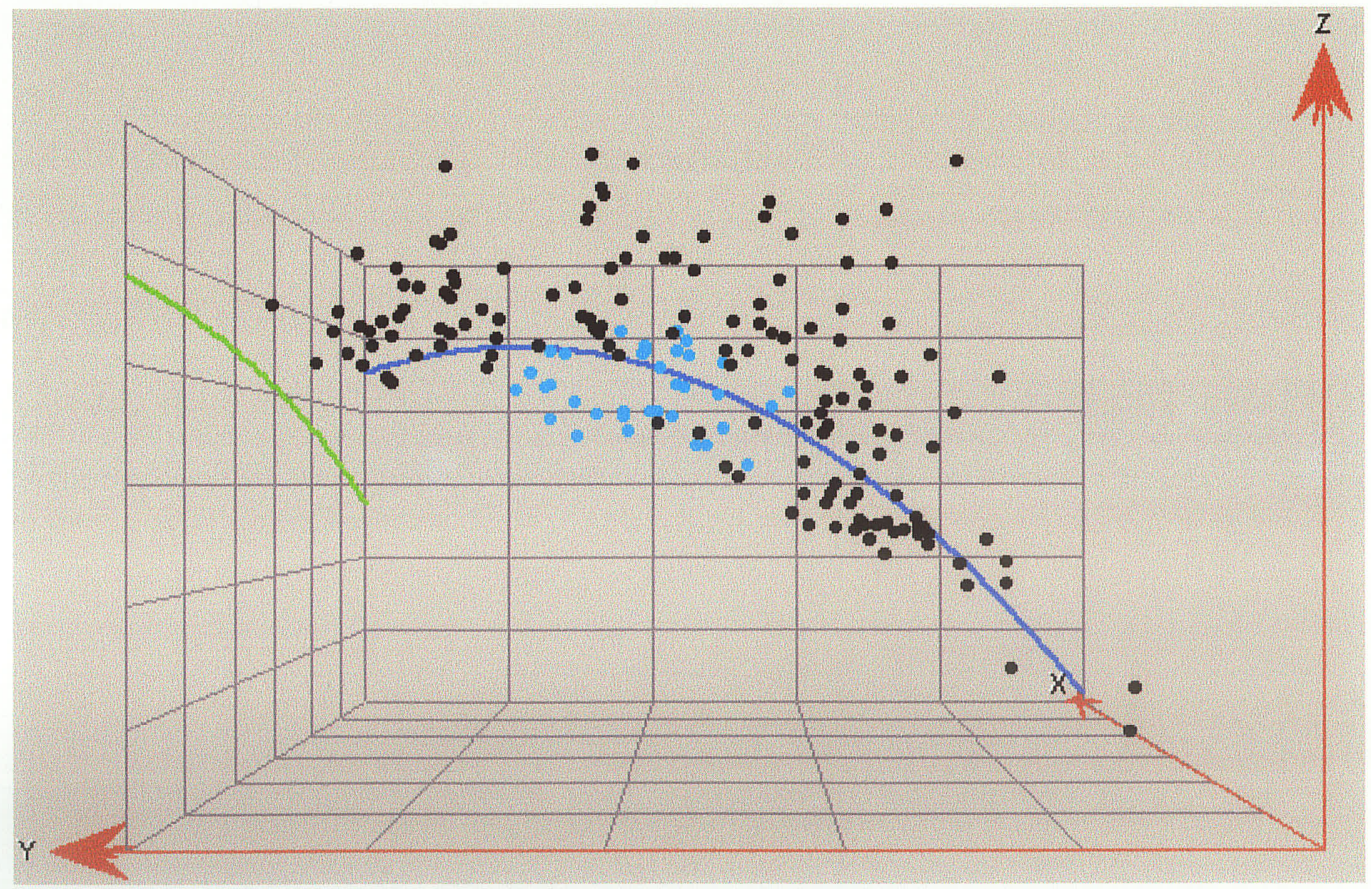

Figure 8: Three-Dimensional Plot - Eight Cluster Solution: Cluster 8-4, Level II/2 


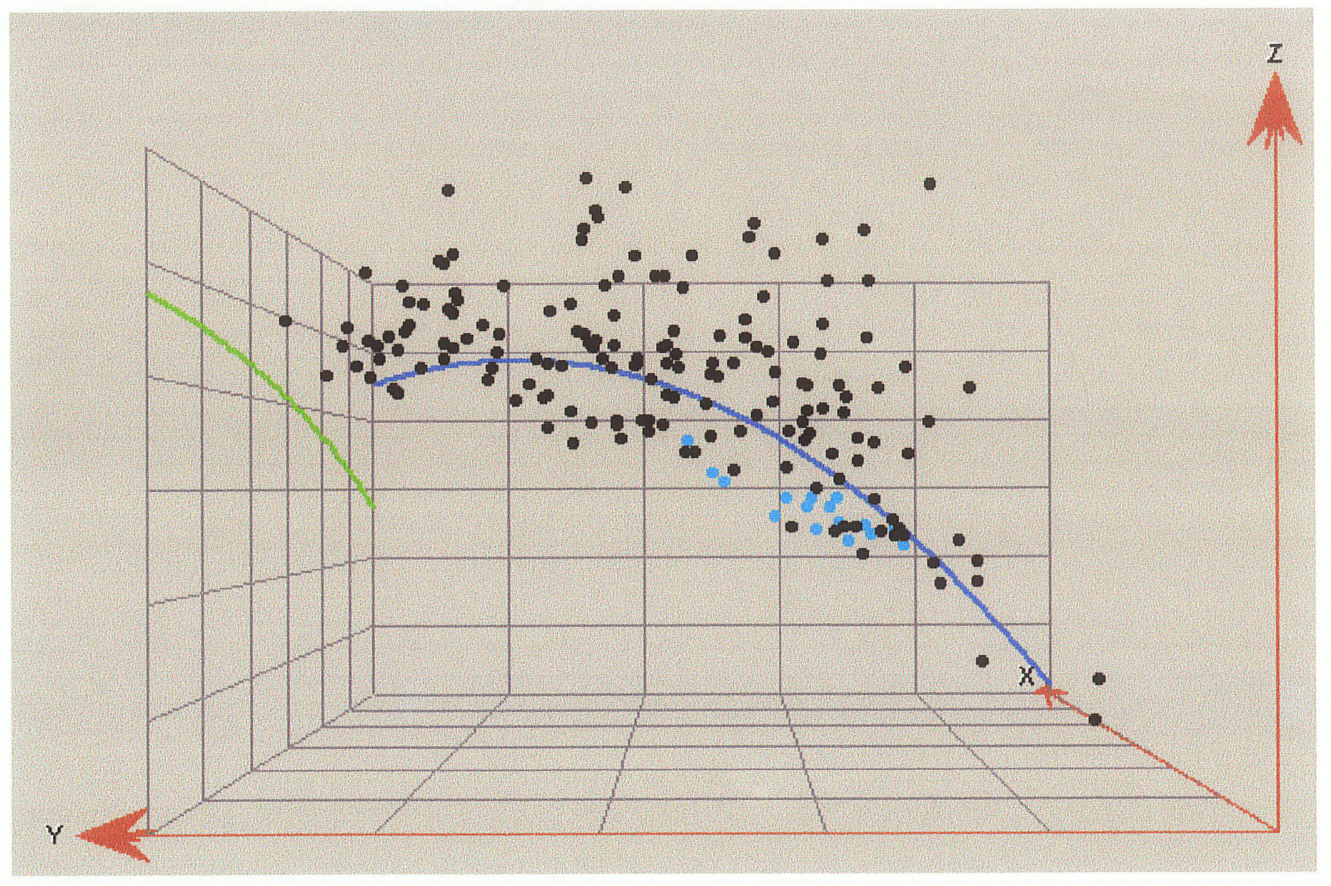

Figure 9: Three-Dimensional Plot - Eight Cluster Solution: Cluster 8-5, Level II/2

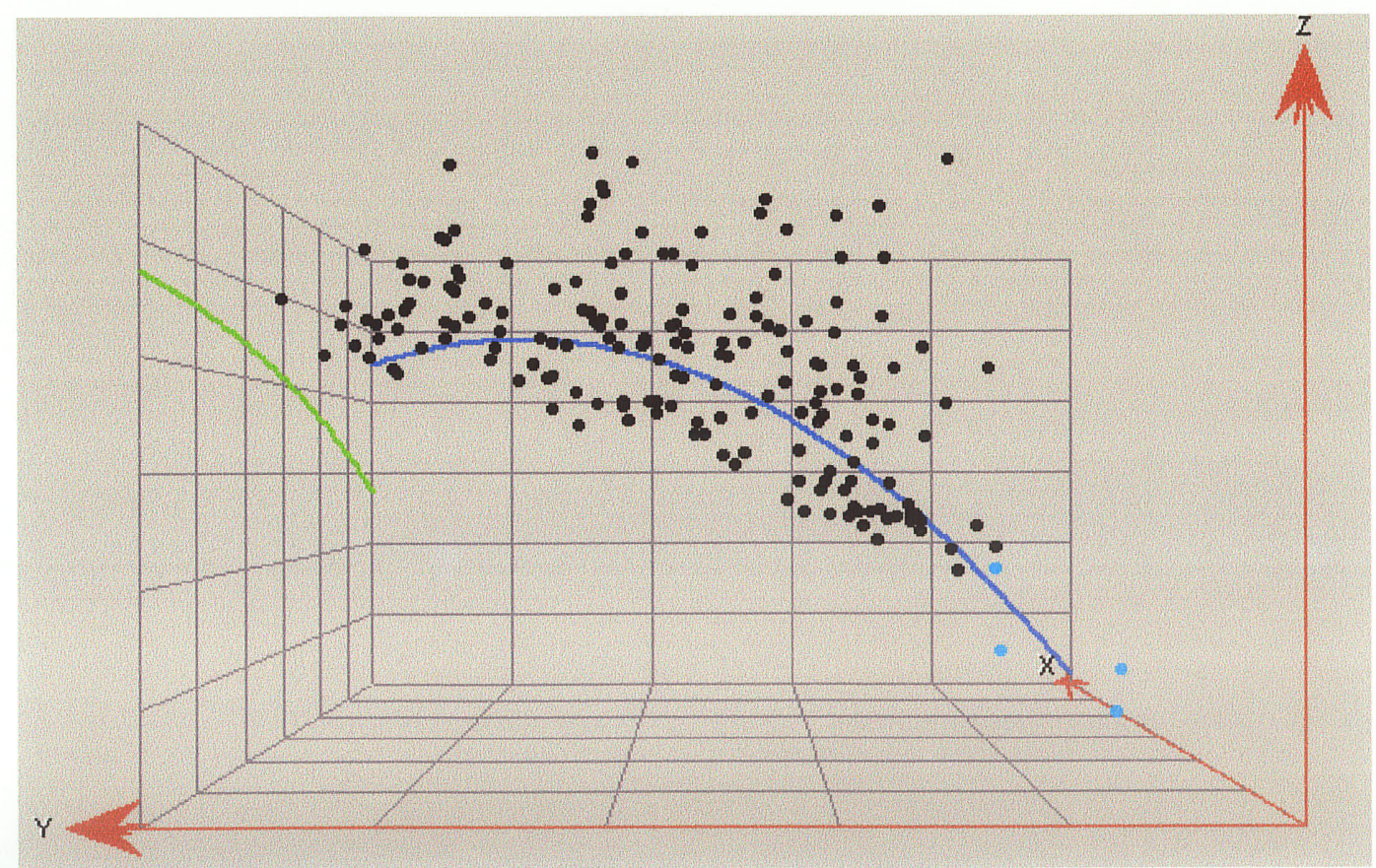

Figure 10: Three-Dimensional Plot - Eight Cluster Solution: Cluster 8-6, Level II/2 


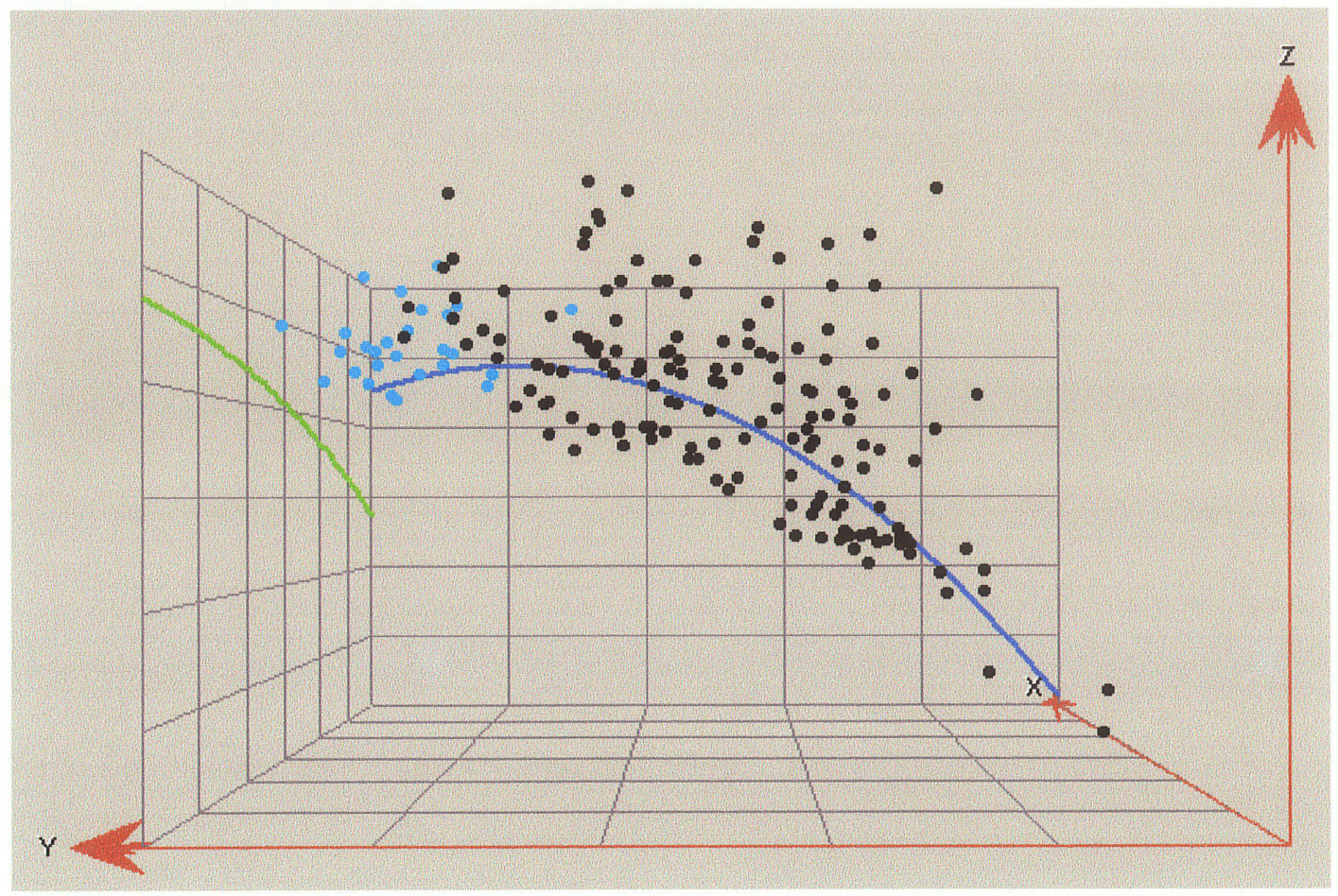

Figure 11: Three-Dimensional Plot - Eight Cluster Solution: Cluster 8-7, Level II/2

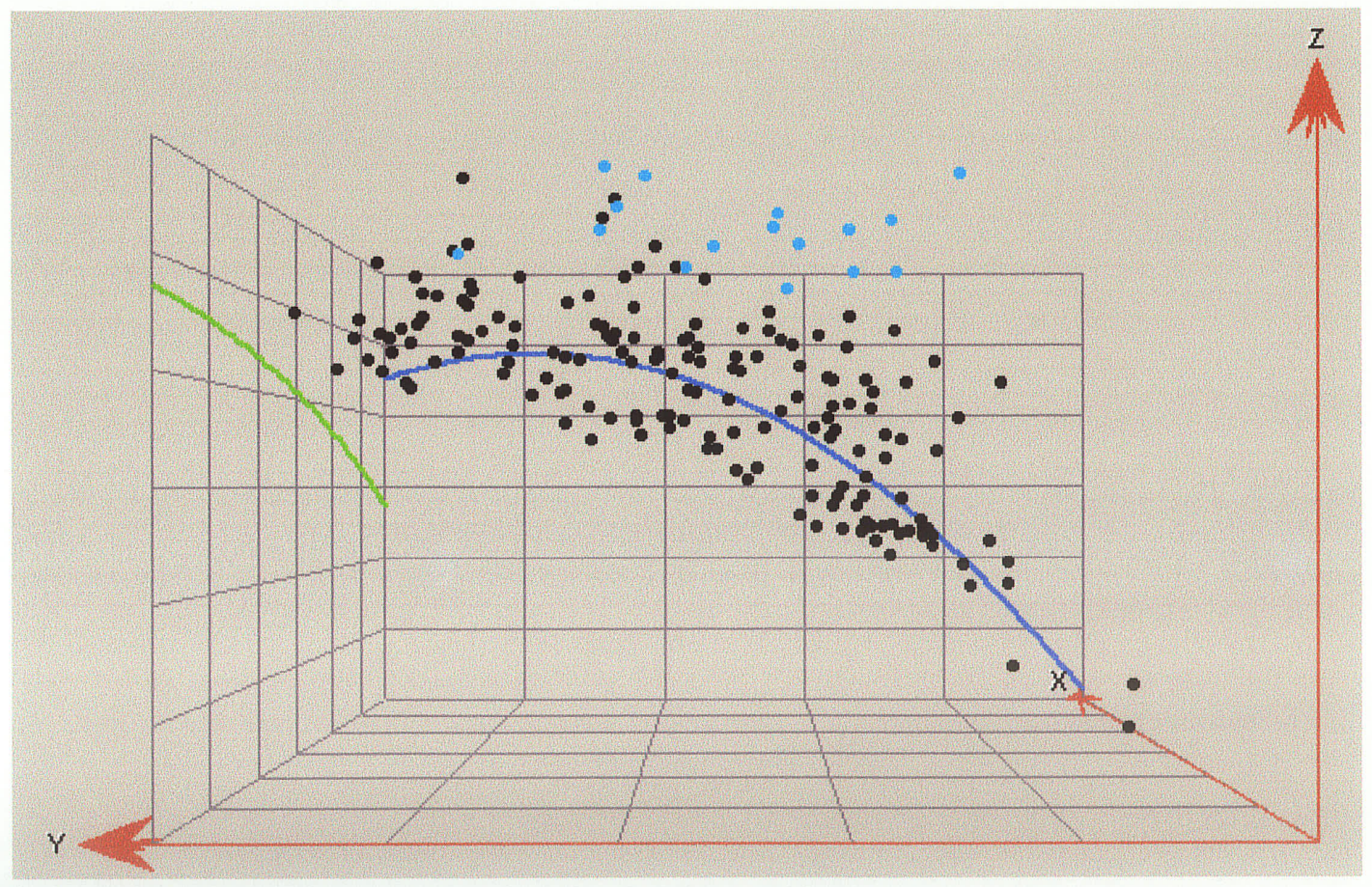

Figure 12: Three-Dimensional Plot - Eight Cluster Solution: Cluster 8-8, Level II/2 


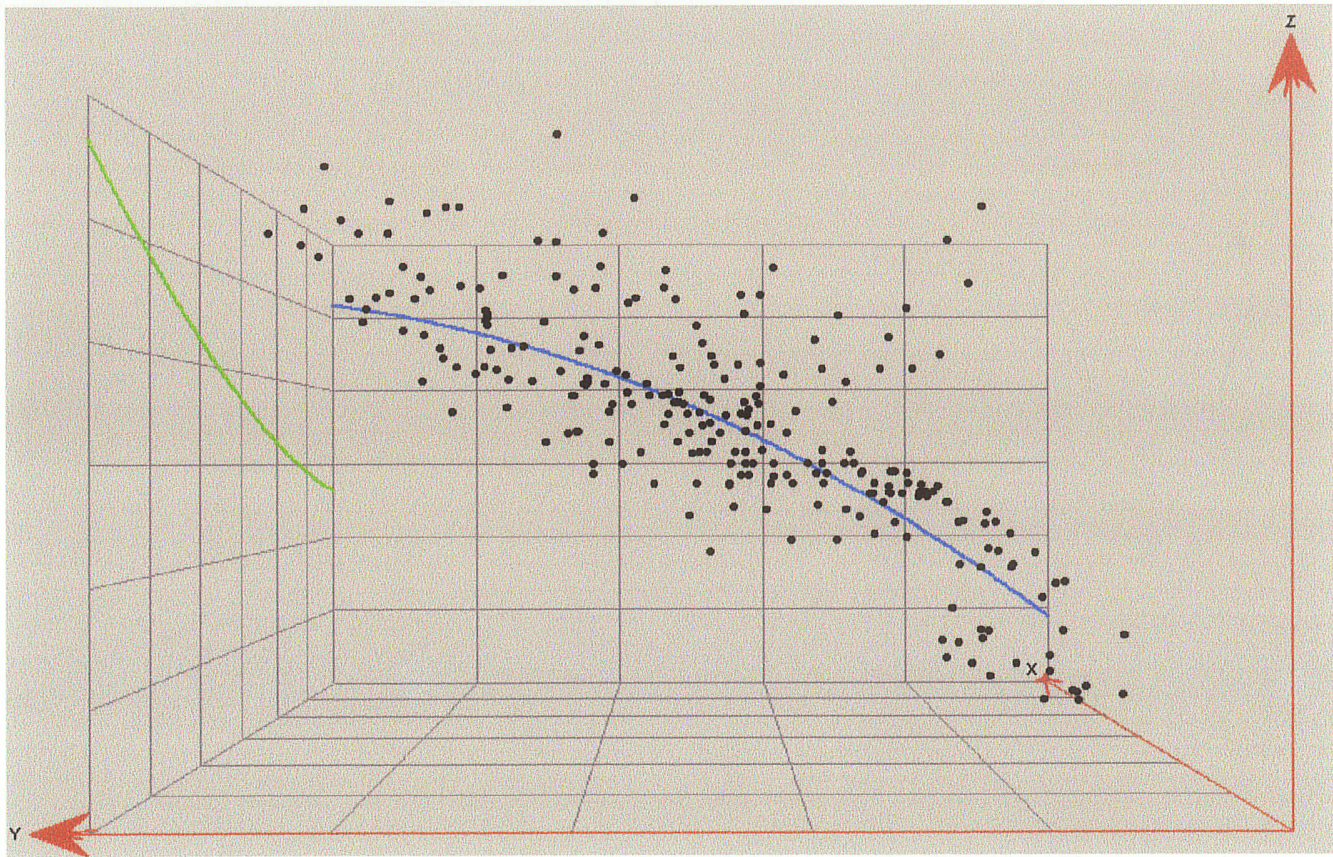

Figure 13: Three-Dimensional Distribution - Level III

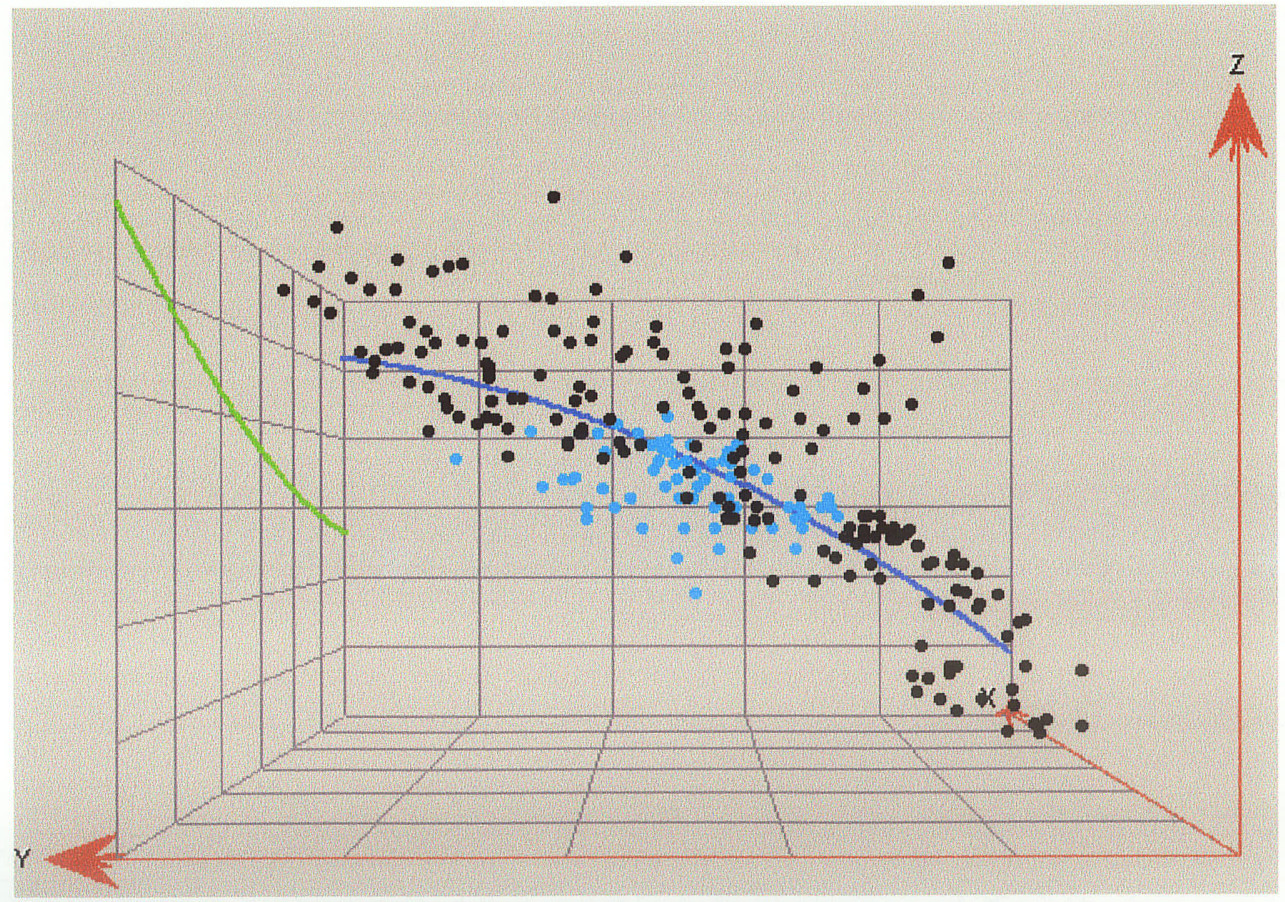

Figure 14: Three-Dimensional Plot - Four Cluster Solution: Cluster 4-1, Level III 


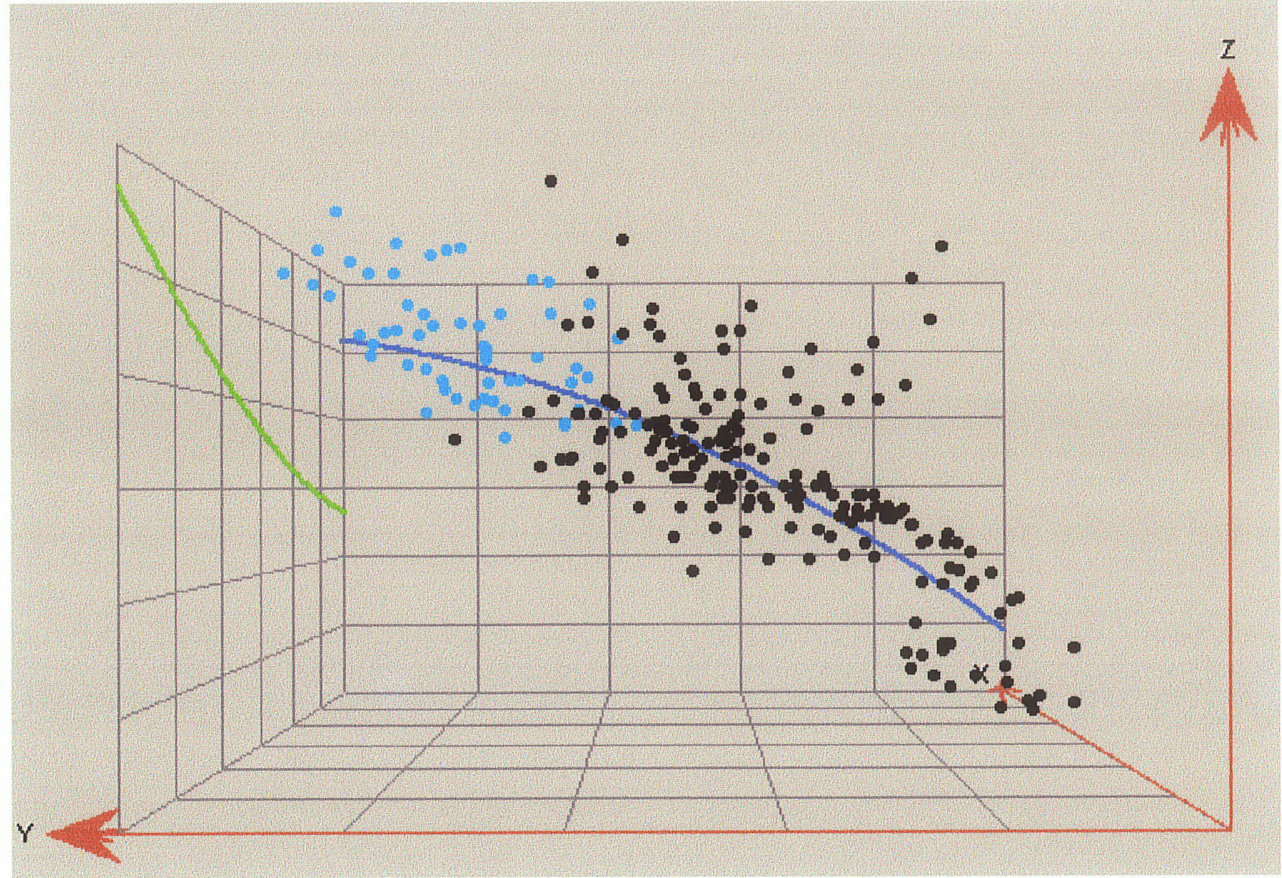

Figure 15: Three-Dimensional Plot - Four Cluster Solution: Cluster 4-2, Level III

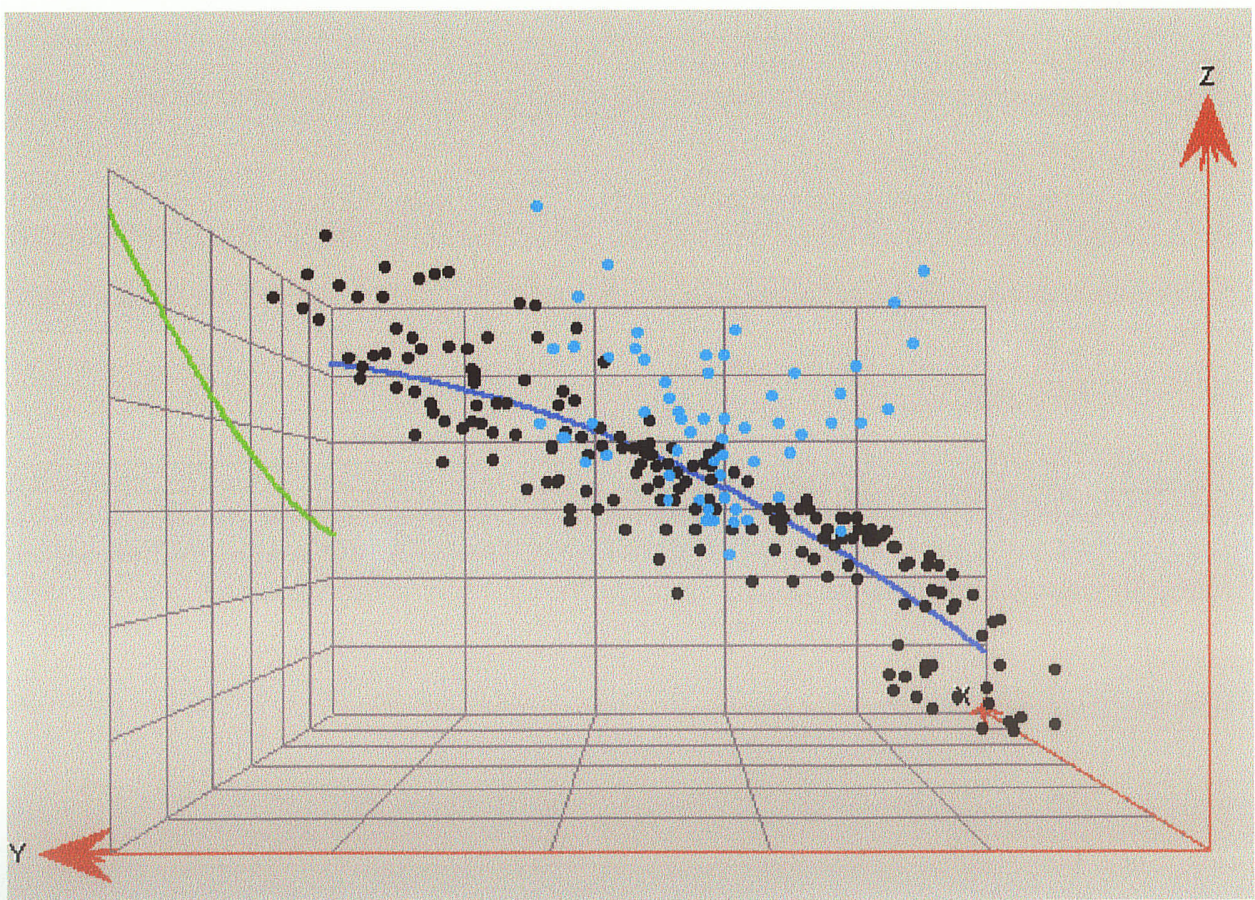

Figure 16: Three-Dimensional Plot - Four Cluster Solution: Cluster 4-3, Level III 


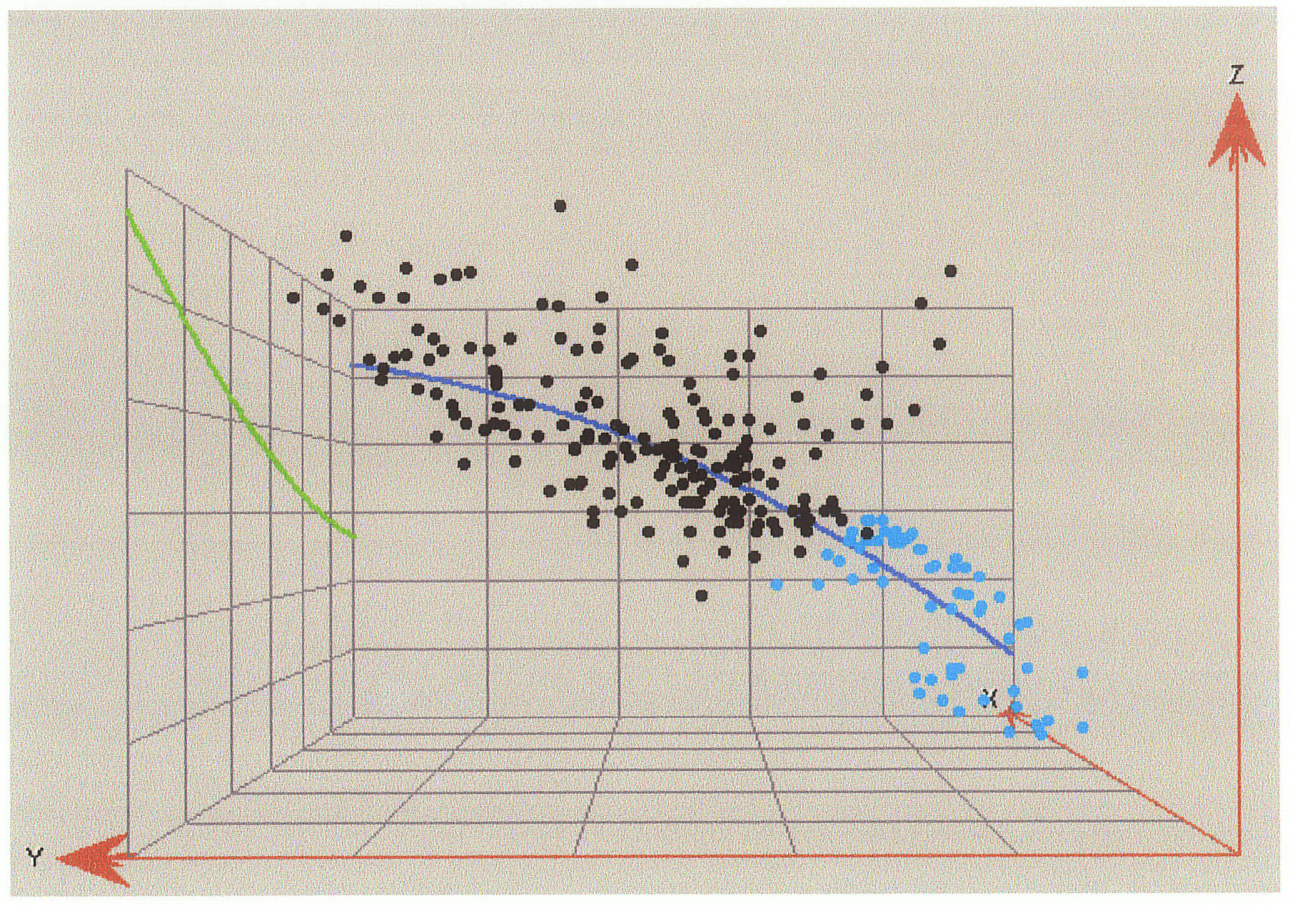

Figure 17: Three-Dimensional Plot - Four Cluster Solution: Cluster 4-4, Level III

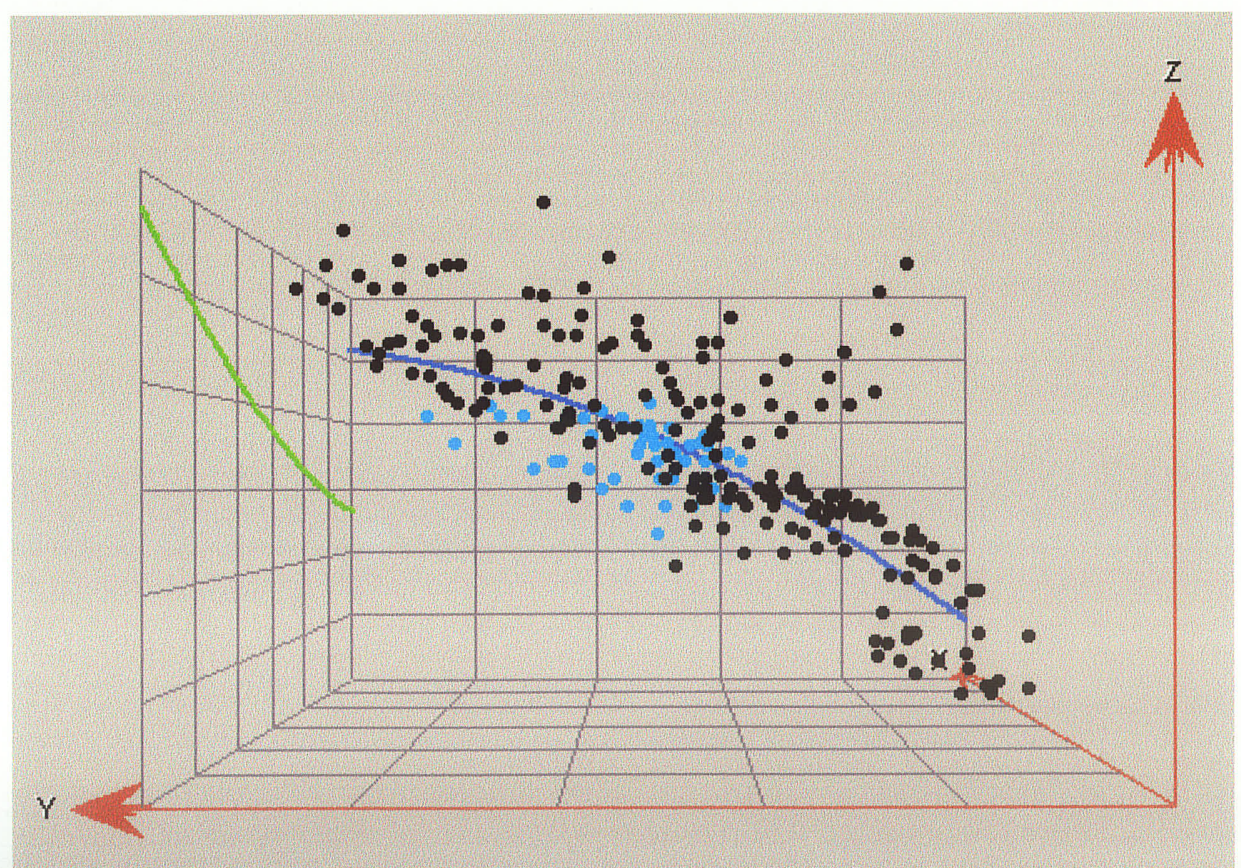

Figure 18: Three-Dimensional Plot - Six Cluster Solution: Cluster 6-1, Level III 


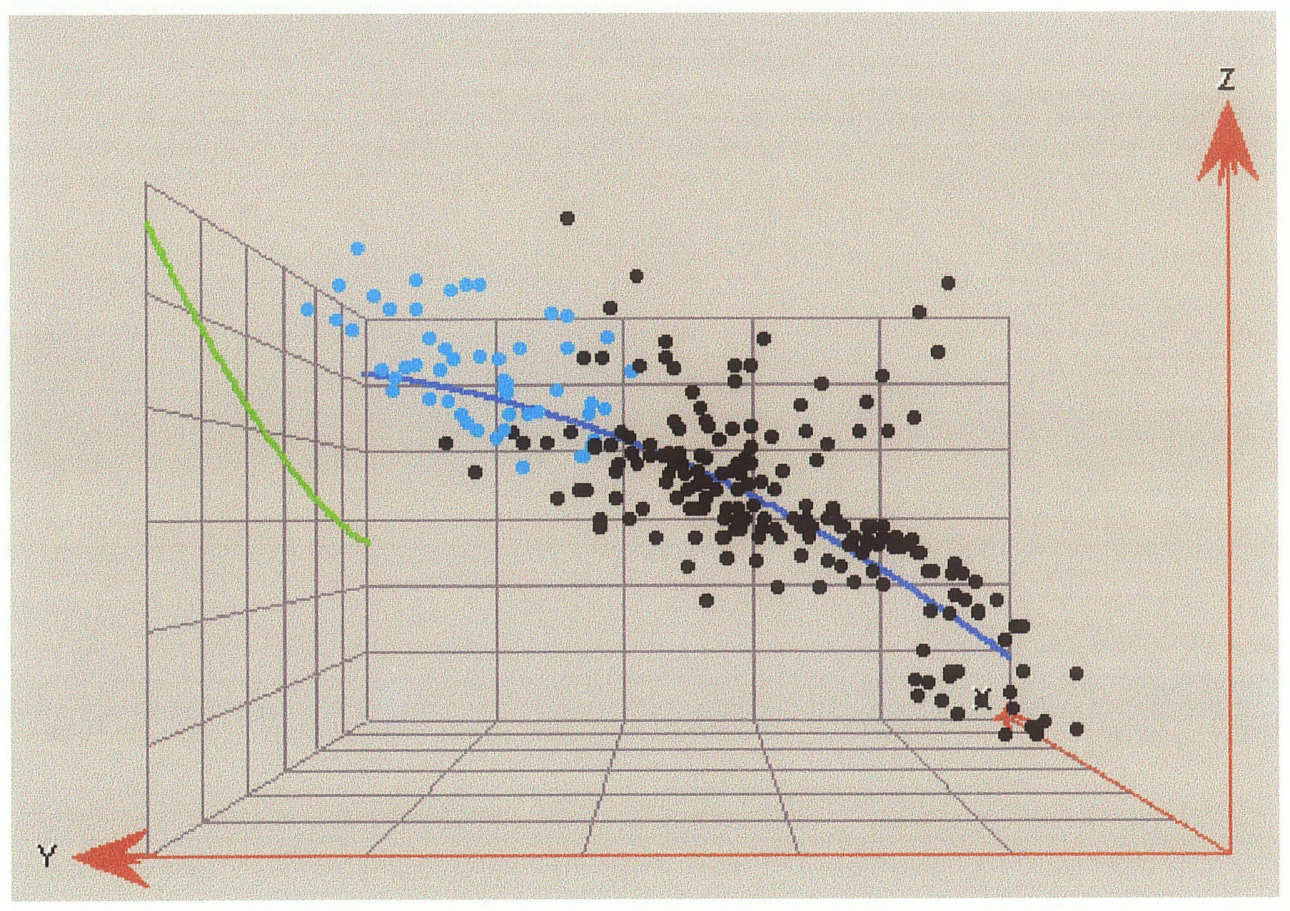

Figure 19: Three-Dimensional Plot - Six Cluster Solution: Cluster 6-2, Level III

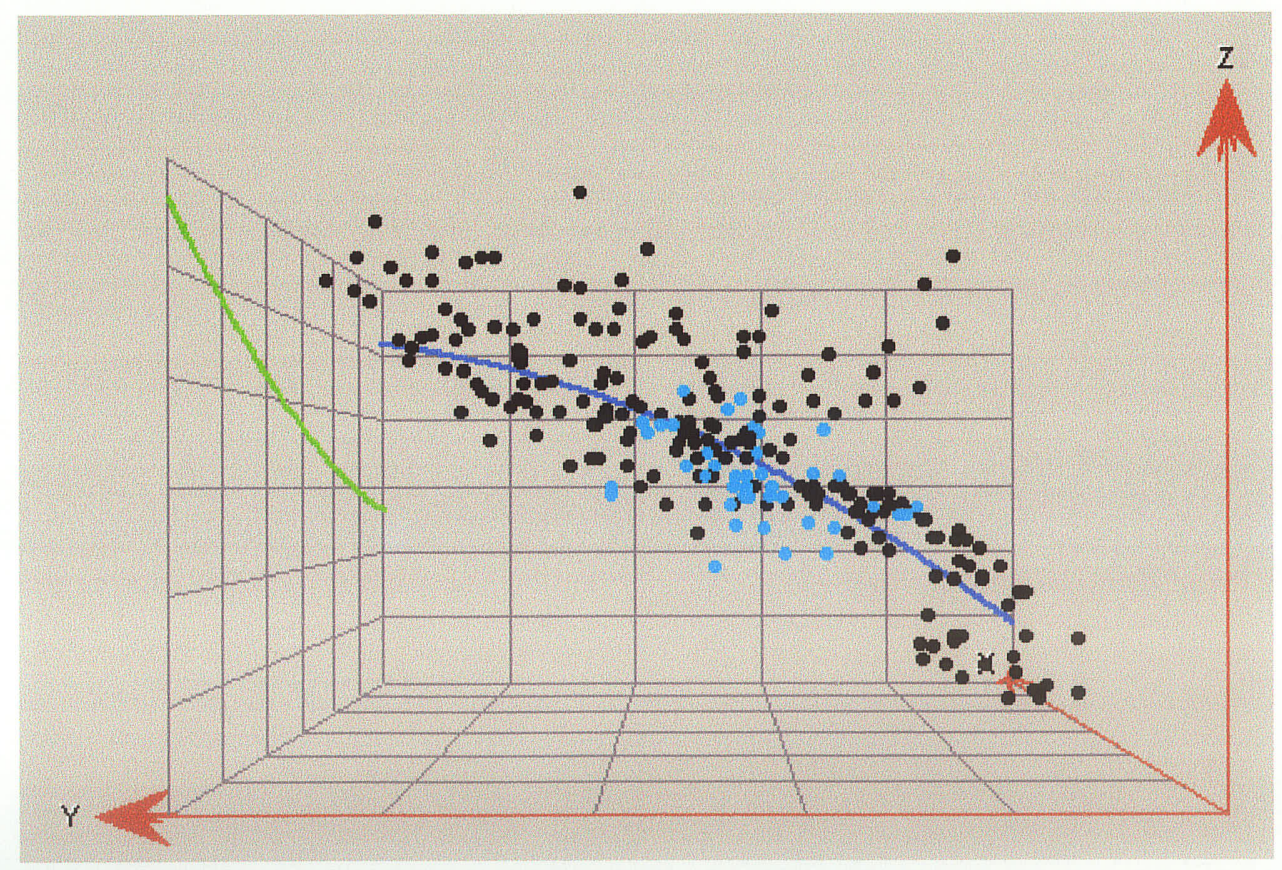

Figure 20: Three-Dimensional Plot - Six Cluster Solution: Cluster 6-3, Level III 


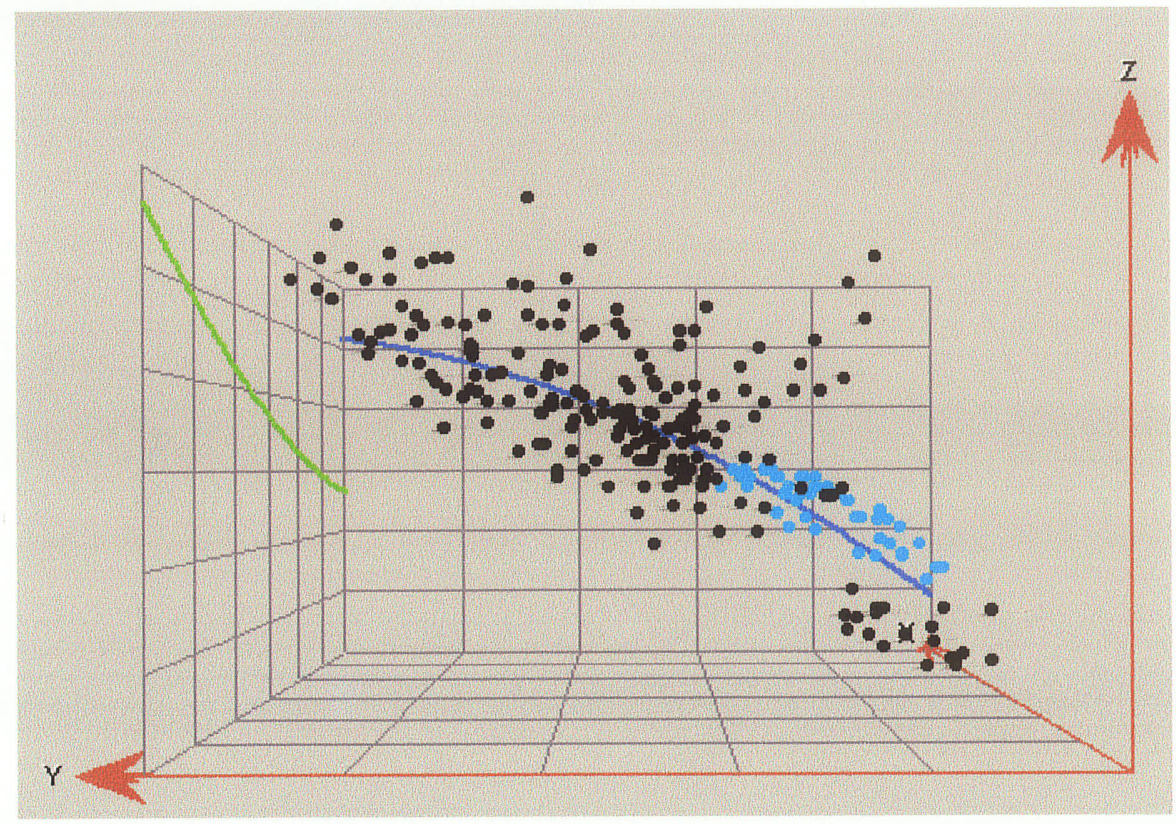

Figure 21: Three-Dimensional Plot - Six Cluster Solution: Cluster 6-4, Level III

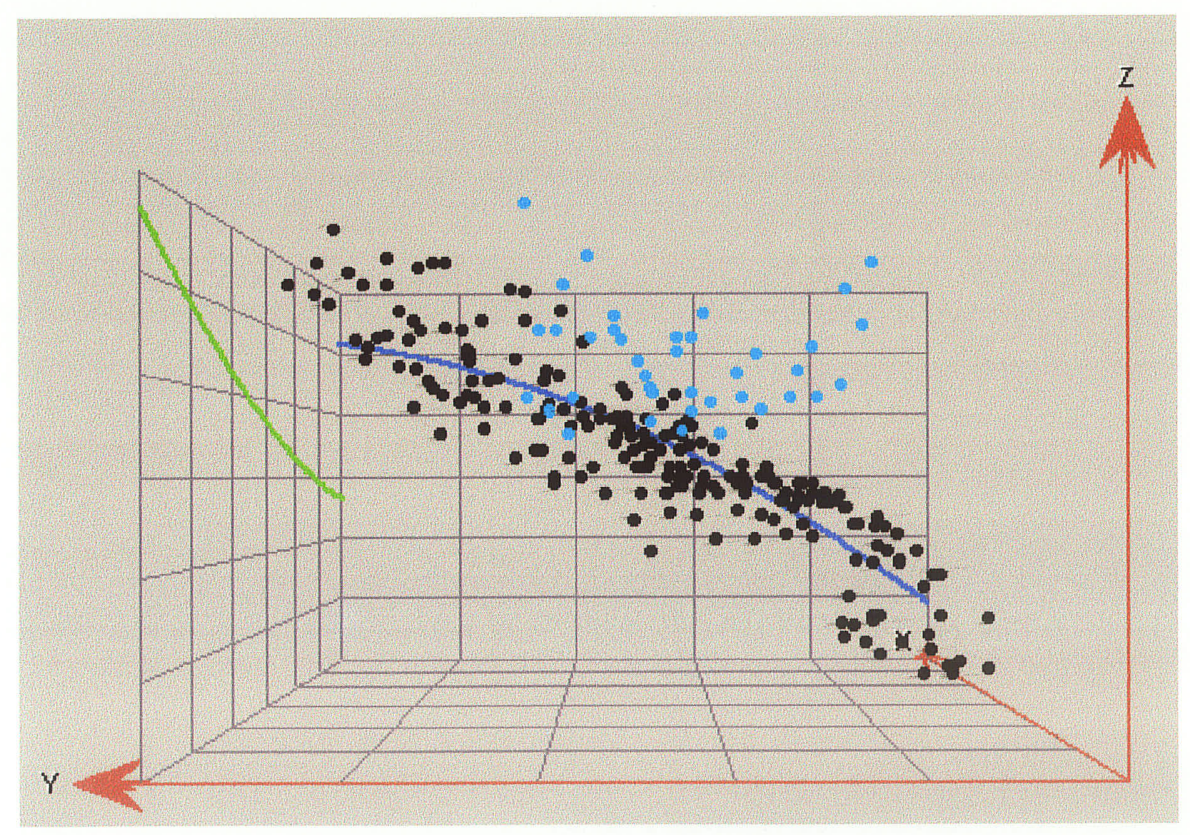

Figure 22: Three-Dimensional Plot - Six Cluster Solution: Cluster 6-5, Level III 


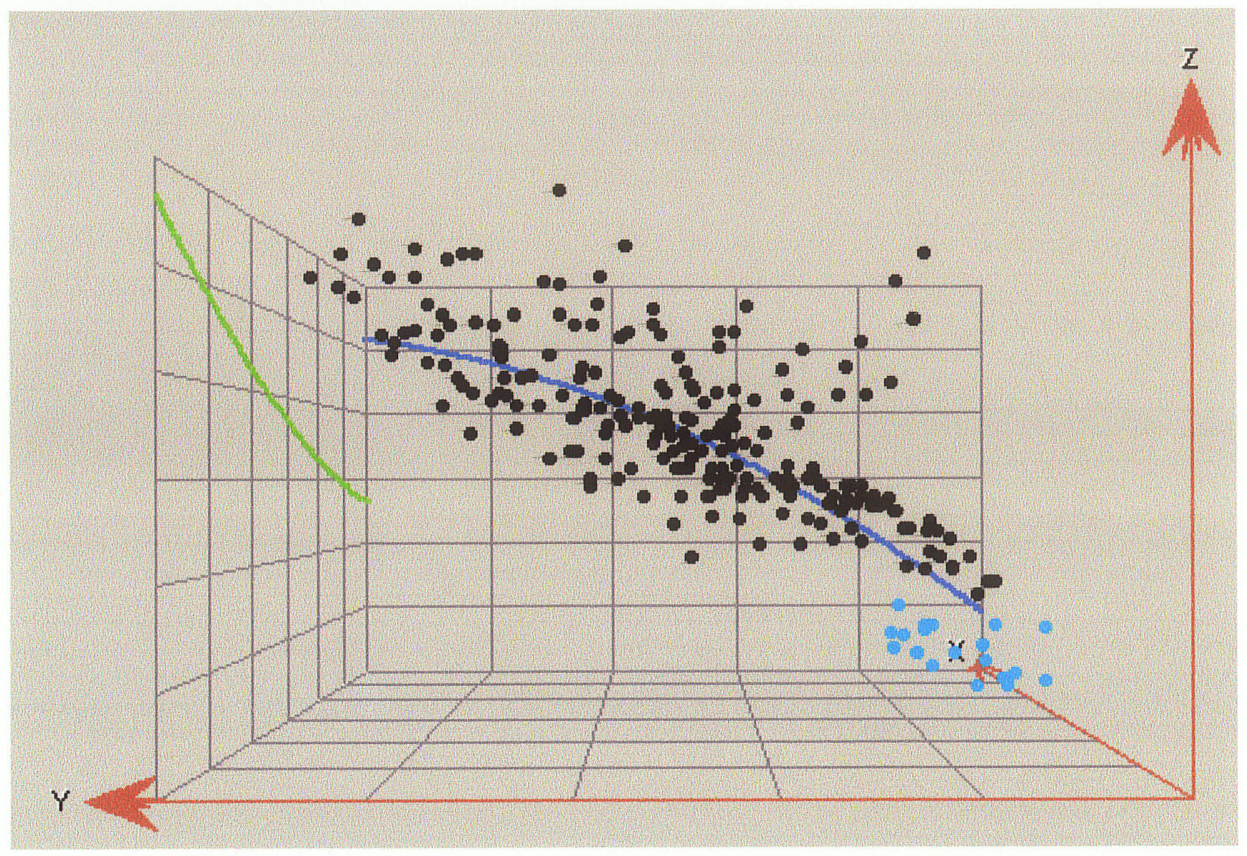

Figure 23: Three-Dimensional Plot - Six Cluster Solution: Cluster 6-6, Level III

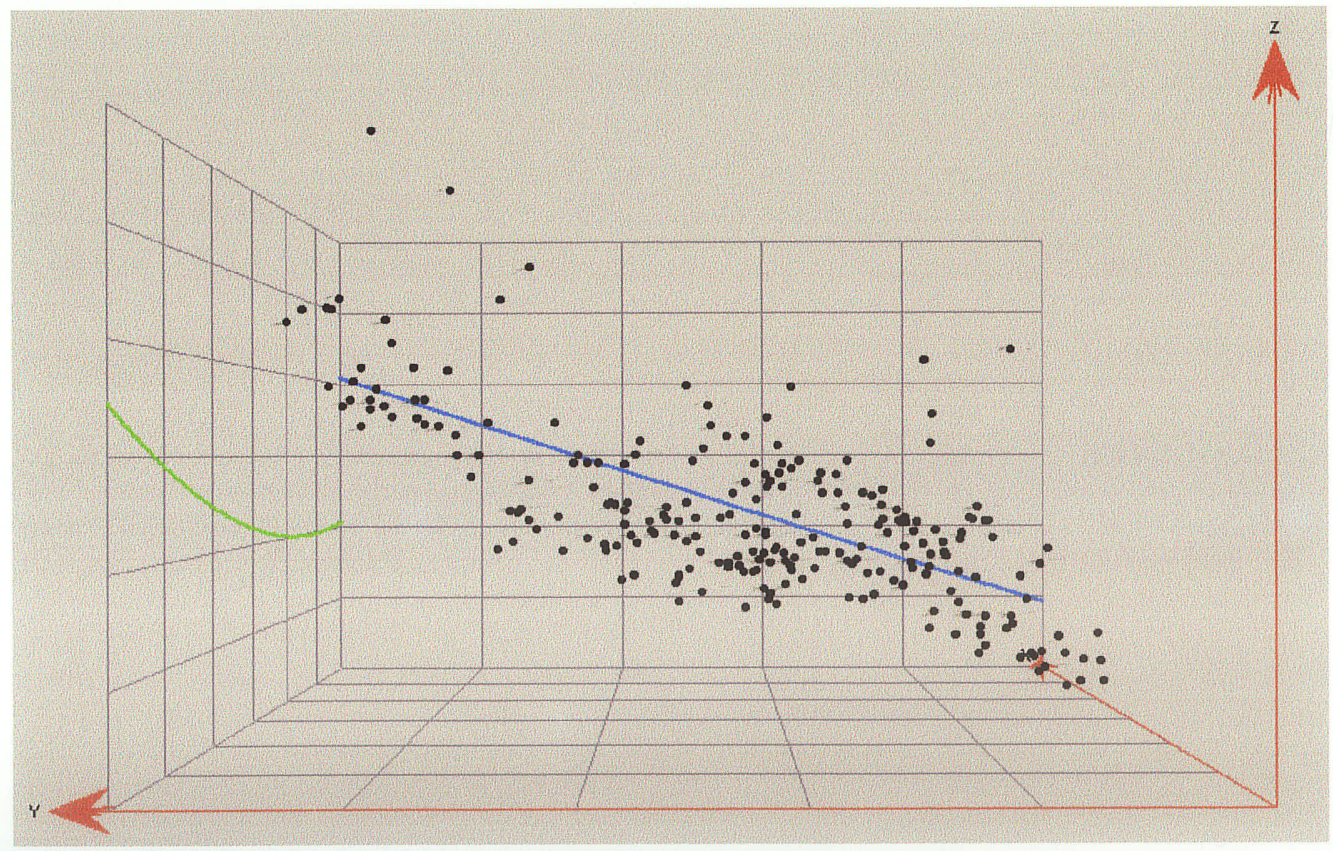

Figure 24: Three-Dimensional Artefact Distribution - Level IV 


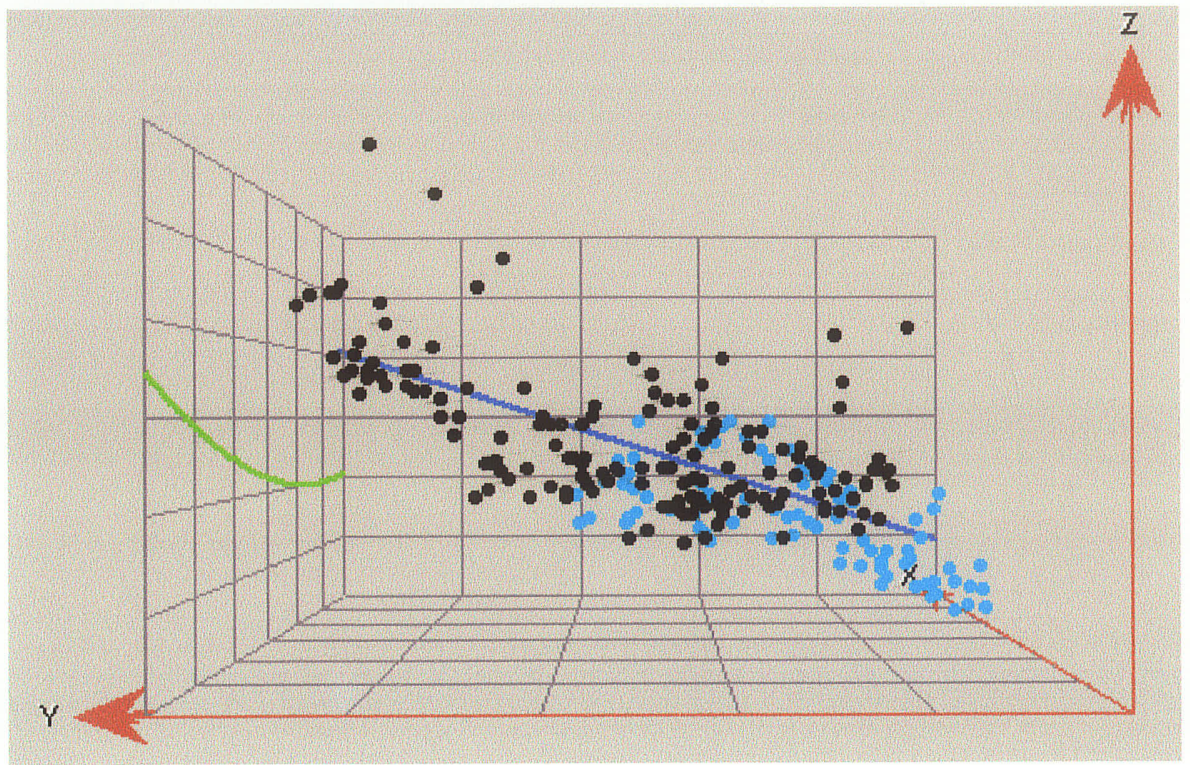

Figure 25: Three-Dimensional Plot - Three Cluster Solution: Cluster 3-1, Level IV

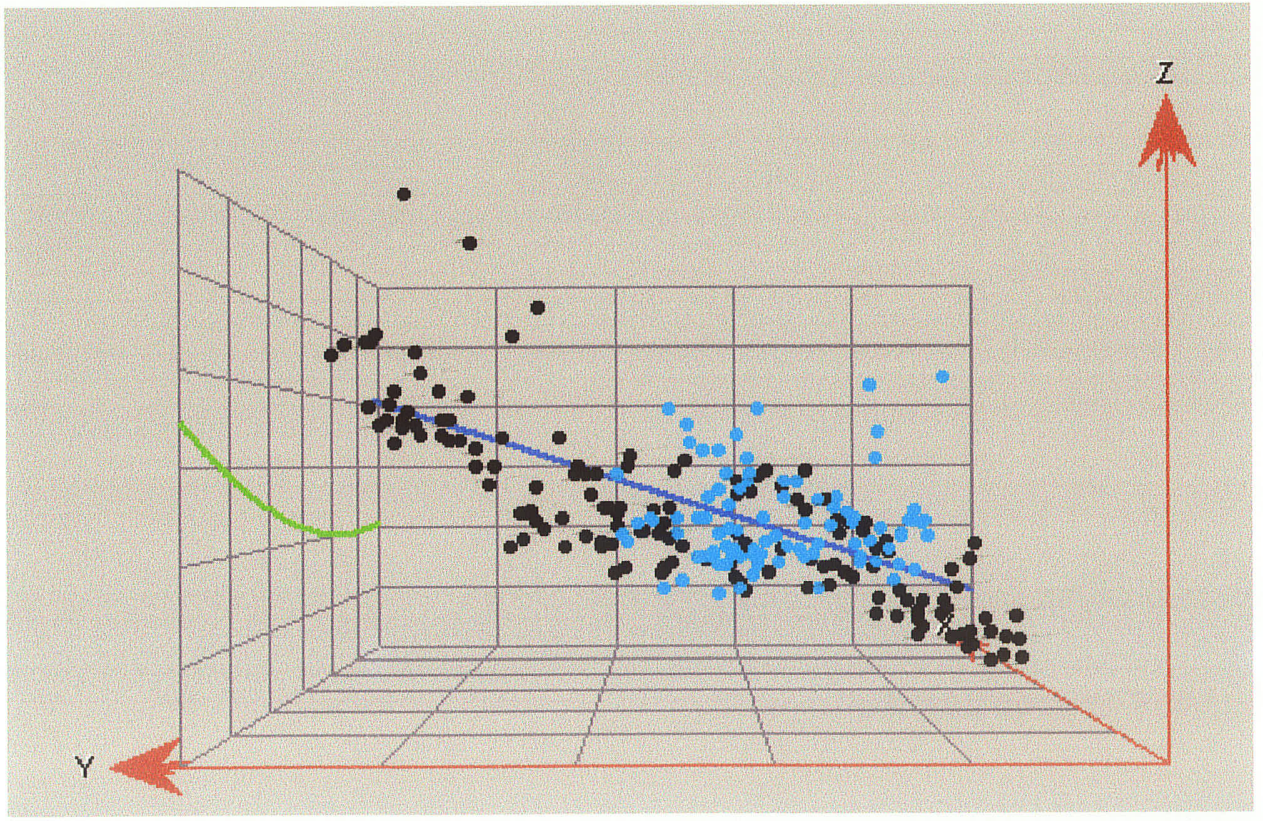

Figure 26: Three-Dimensional Plot - Three Cluster Solution: Cluster 3-2, Level IV 


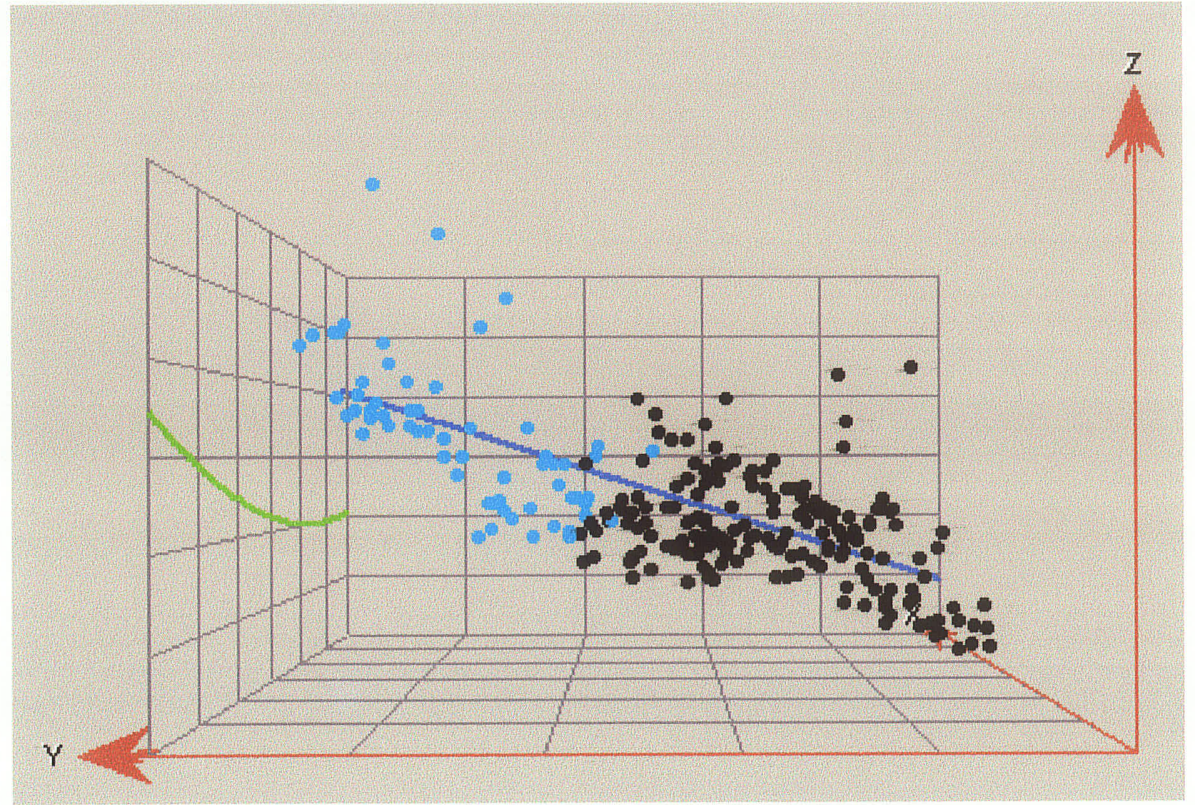

Figure 27: Three-Dimensional Plot - Three Cluster Solution: Cluster 3-3, Level IV

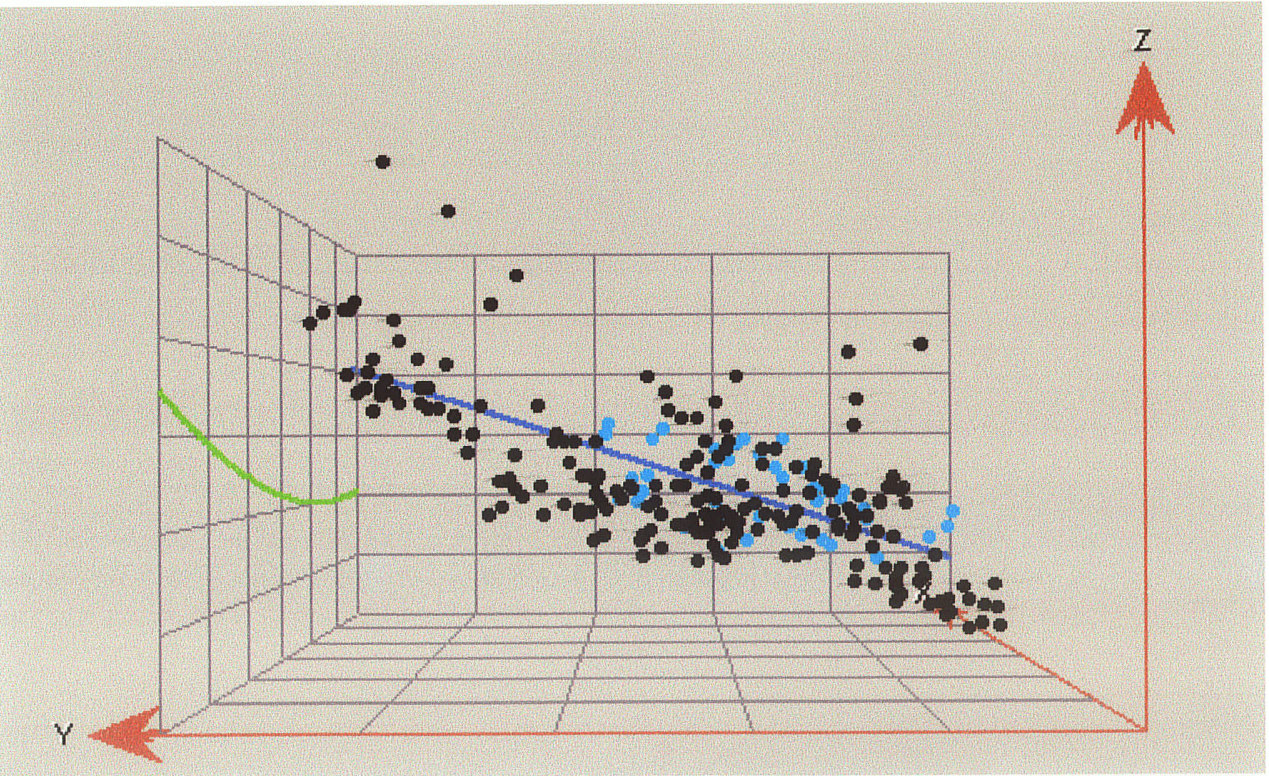

Figure 28: Three-Dimensional Plot - Five Cluster Solution: Cluster 5-1, Level IV 


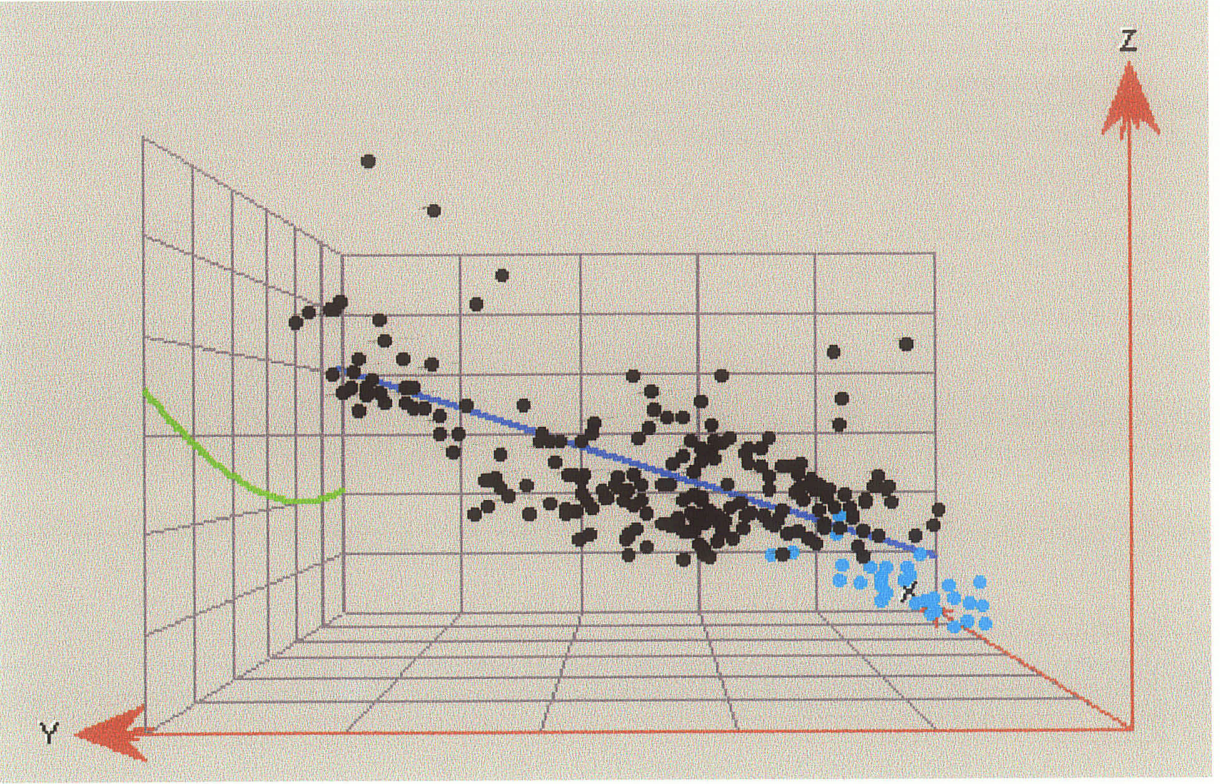

Figure 29: Three-Dimensional Plot - Five Cluster Solution: Cluster 5-2, Level IV

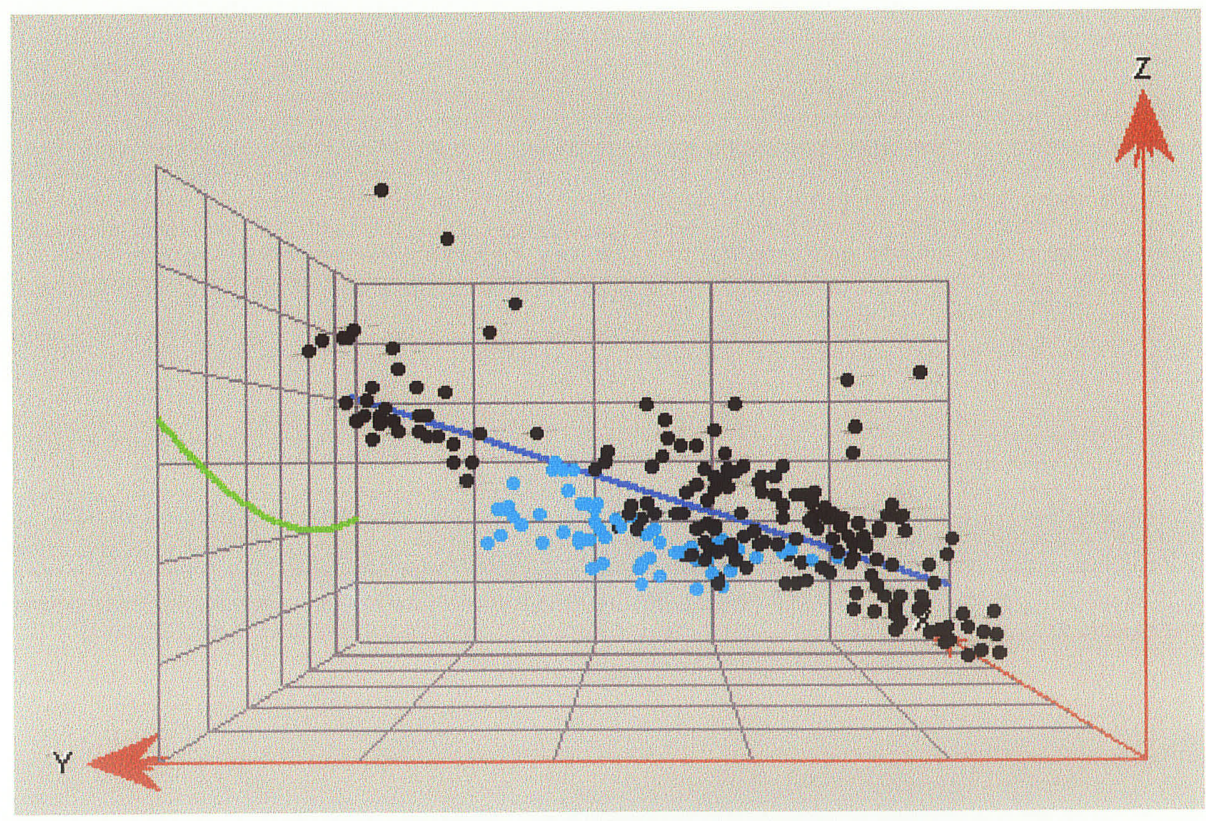

Figure 30: Three-Dimensional Plot - Five Cluster Solution: Cluster 5-3, Level IV 


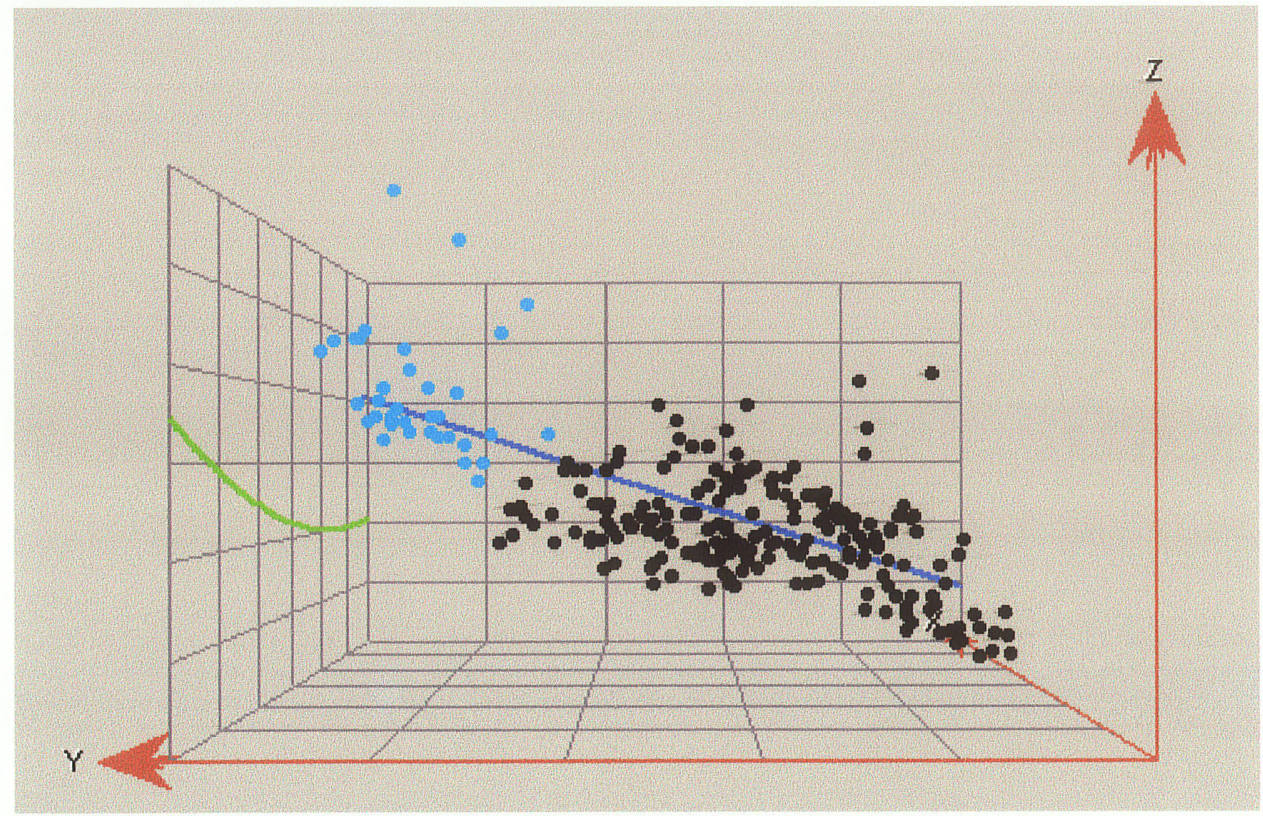

Figure 31: Three-Dimensional Plot - Five Cluster Solution: Cluster 5-4, Level IV

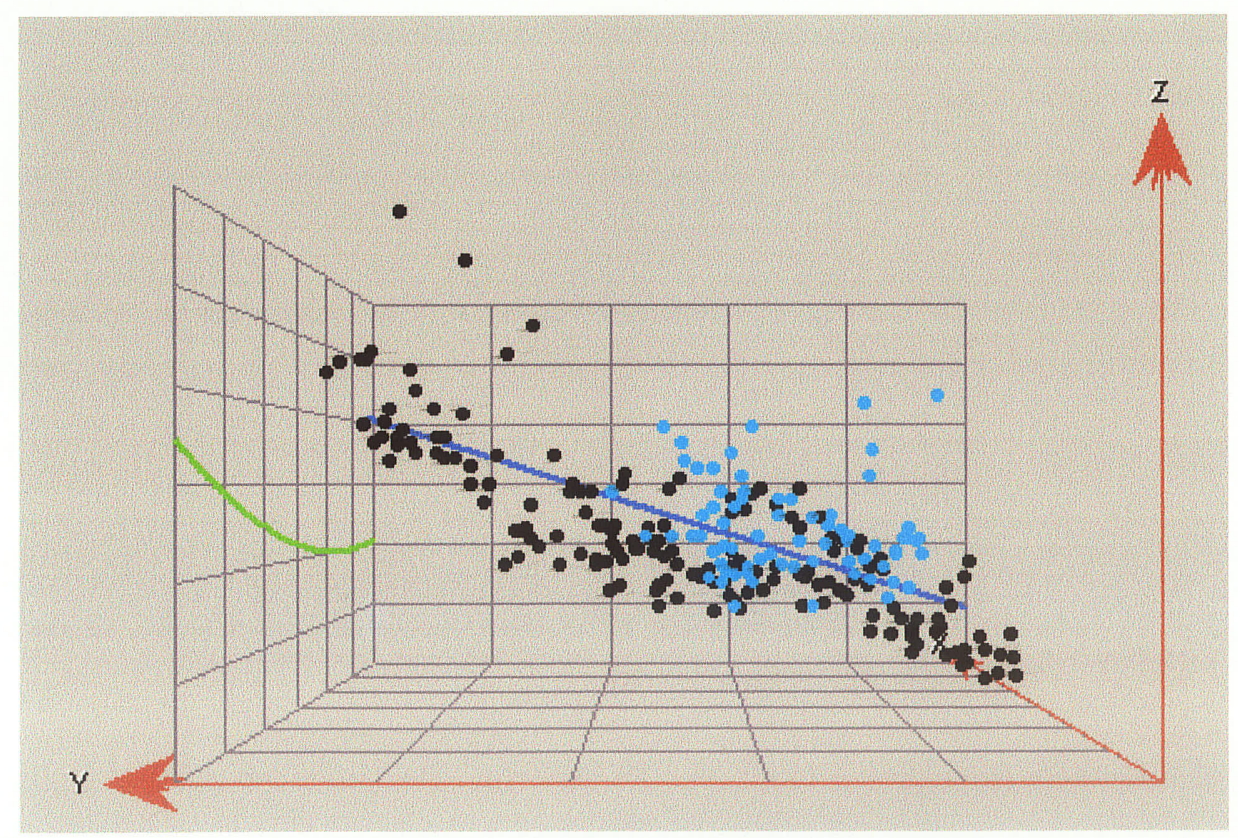

Figure 32: Three-Dimensional Plot - Five Cluster Solution: Cluster 5-5, Level IV 
- Bibliography - 


\section{- Bibliography -}

Allen, K. M. S., S. W. Green

1990 Interpreting Space. In Interpreting space: GIS and Archaeology. K. M. S.

Allen, S. W. Green and E. B. W. Zubrow. London, Taylor and Francis: 383-386.

Altschul, J. H.

1990 Red Flag Models: the use of modelling in Management Contexts. In

Interpreting Space: GIS and Archaeology. K. M. S. Allen, S. W. Green and E. B. W. Zubrow, Eds. Taylor and Francis, London: 226-238.

Ammerman, A. J., K. W. Kintigh and J. F. Simek

1983 Recent Developments in the Application of the K-means Approach to Spatial Analysis. In The Human Uses of Flint and Chert: Proceedings of the Fourth International Flint Symposium Held at Brighton Polytechnic, G. D. G. Sieveking and M. H. Newcomer, Eds. Cambridge University Press, Cambridge: 211-216.

Belcher, R. C. and A. Paradis

1992 A Mapping Approach to Three-Dimensional Modeling. In ThreeDimensional Modeling with Geoscientific Information Systems, A. K. Turner, Ed. Kluwer Academic Publishers, Netherlands: 107-122.

Berry, K. J., K. L. Kvamme and P. W. Mielke Jr 1980 A Permutation Technique for the Spatial Analysis of the Distribution of Artifacts into Classes. American Antiquity 45(1): 55-59.

Binford, L. R. (Editor)

1977 For Theory Building in Archaeology: Essays on Faunal Remains, Aquatic Resources, Spatial Analysis and Systemic Modelling. Academic Press, New York.

1978 Dimensional Analysis of Behaviour and Site Structure: Learning from an Eskimo Hunting Stand. American Antiquity 43(3): 330-361.

1996 Hearth and Home: The Spatial Analysis of Ethnographically Documented Rock Shelter Occupations as a Template for Distinguishing Between Human and Hominid Use of Sheltered Space. In Middle Palaeolithic and Middle Stone Age Settlement Systems. Nicholas J. Conard and Fred Wendorf, Eds. XII International Congress of Prehistoric and Protohistoric Sciences. Forli-Italia: 229-235.

Blankholm, H. P. 1991 Intrasite Spatial Analysis in Archaeology: Past and Current Trends. Aarhus University Press, Denmark 
Booth, B.

2000 Using ArcGIS 3D Analyst: GIS by ESRI. Environmental Systems Research Institute, Inc. United States.

Burke, A.

In Press Karabi Tamchin: Faunal Remains. In The Palaeolithic of Crimea: The Middle and Early Upper Palaeolithic of Eastern Crimea, Vol. 3. V. Chabai and A. Marks, Eds. ERAUL, Liege: Chapter 16.

Burrough, P. A.

1986 Principles of Geographic Information Systems for Land Resources Assessment. Clarendon Press, Oxford, England.

Carr, C.

1984 The Nature of Organization of Intrasite Archaeological Records and Spatial Analytical Approaches to Their Investigation. In Advances in Archaeological Method and Theory, Vol. 7. M. B. Schiffer, Ed. Academic Press, New York: 103-211.

1987 Dissecting Intrasite Artifact Palimpsests Using Fourier Methods. In Method and Theory for Activity Area Research: An Ethnoarchaeological Approach, S. Kent, Ed. Columbia University Press, New York: 236-291.

Chabai, V.P.

1998 The History of Crimean Middle Palaeolithic Studies. In The Middle Palaeolithic of Western Crimea, Vol. 1 of The Palaeolithic of Crimea, I. ERAUL 84, Liege: 1-15.

Chabai, V.P. and K. Monigal (Editors)

1999 The Middle Palaeolithic of Western Crimea. Vol. 2 of The Palaeolithic of Crimea, II. ERAUL 87, Liege.

Clark, P.J. and F.C. Evans

1954 Distance to Nearest Neighbour as a Measure of Spatial Relationships in Populations. Ecology 35:445-453.

Clarke, D. L. (Editor)

1977 Spatial Archaeology. Academic Press, London.

1979 Spatial Information in Archaeology. In Analytical Archaeology, pp. 453482. Academic Press, New York. 
Cowgill, G. L.

1968 Archaeological Applications of Factor, Cluster and Proximity Analysis. American

Antiquity 33(3): 367-375.

Csaki, G., E. Jerem and F. Redo

1995 Data Recording and GIS Applications in Landscape and Intra-site Analysis: Case Studies in Progress at the Archaeological Institute of the Hungarian Academy of Sciences. In Archaeology and Geographical Information Systems: A European Perspective, G. Lock and Z. Stancic, Eds. Taylor and Francis, London: 85-100.

Dacey, M. F.

1973 Statistical Tests of Spatial Association in the Locations of Tool Types. American Antiquity 38(3): 320-328.

Dalla Bona, L.

1993 A Predictive Model Prehistoric Activity Location for the Souris

River, Saskatchewan. Department of Anthropology. University of

Manitoba, Winnipeg, Manitoba.

Djindjian, F.

1988 Improvements in Intra-Site Spatial Analysis Techniques. In Computer and Quantitative Methods in Archaeology 1988, Vol.1. S. P. Q. Rahtz, Ed. BAR International Series 446, Oxford, England: 95-106.

Durand, S. R. and L. C. Pippin 1992 A Pragmatic Application of the Nearest Neighbour Statistic. Journal of Field Archaeology 19:263-271.

Ebert, D.

2002 Predictive Modeling and the Ecology of Hunter-Gatherers of the Boreal Forest of Manitoba. $\mathrm{PhD}$ Dissertation. Department of Anthropology, University of Manitoba, Winnipeg, Manitoba.

Enloe, J. G., F. David and T. S. Hare

1994 Patterns of Faunal Processing at Section 27 of Pincevent: The Use of Spatial Analysis and Ethnoarchaeological Data in the Interpretation of Archaeological Site Structure. Journal of Anthropological Archaeology 13: 105 124.

Farizy, C.

1994 Spatial Patterning of Middle Palaeolithic Sites. Journal of Anthropological Archaeology 13: 153-160. 
Ferring, C. R.

1984 Intrasite Spatial Patterning: Its Role in Settlement - Subsistence Systems

Analysis. In Intrasite Spatial Analysis in Archaeology, H. Hietala, Ed. New

Directions in Archaeology. Cambridge University Press, Cambridge: 116-126

Fisher, P.F.

1999 Geographical Information Systems: Today and Tomorrow? In

Geographical Information Systems and Landscape Archaeology. Mark Gillings,

David Mattingly and Jan van Dalen, Eds. Oxbow Books: 5-11.

Gaffney, V. and P. M. van Leusen

1995 Postscript - GIS, Environmental Determinism and Archaeology. In

Archaeology and Geographical Information Systems: A European Perspective. G.

Lock and Z. Stancic, Eds. Taylor and Francis, London: 367-382.

Galanidou, N.

2000 Patterns in Caves: Foragers, Horticulturists and the Use of Space. Journal of Anthropological Archaeology 19: 243-275.

Getter, J. R.

1986 Indian Integrated Resource Information Program (IIRIP). In Geographic

Information Systems in Government Vol. 1. B. K. Opitz, Ed. A. Deepak

Publishing, Hampton, Virginia: 141-146.

Gilbert, C.

1991 Cultural Landscapes and the New Technologies. The Public Historian 13(3): 109-112.

Graham, I.

1980 Spectral Analysis and Distance Methods in the Study of Archaeological Distributions. Journal of Archaeological Science 7:105-129.

Green, S.

1990 Approaching Archaeological Space, In Interpreting Space: GIS and Archaeology. Kathleen M.S. Allen, Stanton W. Green and Ezra B.W. Zubrow, Eds. London: Taylor and Francis: 3-8.

Gregg, S. A., K. W. Kintigh and W. Robert 1991 Linking Ethnoarchaeological Interpretation and Archaeological Data - The Sensitivity of Spatial Analytical Methods to Postdepositional Disturbance. In The Interpretation of Archaeological Spatial Patterning, E. M. Kroll and T. D. Price, Eds. Plenum Press, New York: 149-198. 
Guillot, D. and G. Leroy

1995 The use of GIS for Archaeological Resource Management in France: the SCALA Project, with a Case Study in Picardie. In Archaeology and Geographical Information Systems: A European Perspective. G. R. Lock and Z. Stancic, Eds. Taylor and Francis, London: 15-26.

Harris, T. M. and G. R. Lock 1995 Toward an Evaluation of GIS in European Archaeology: the Past, Present and Future of Theory and Applications. In Archaeology and Geographical Information Systems: A European Perspective. G. R. Lock and Z. Stancic, Eds. Taylor and Francis, London: 349-365.

Hietala, H. J. (editor) 1984 Intrasite Spatial Analysis in Archaeology. Cambridge University Press, Cambridge.

Hietala, H. J. and D. E. Stevens 1977 Spatial Analysis: Multiple Procedures in Pattern Recognition Studies. American Antiquity 42(4): 539-559.

Hodder, I. And E. Okell

1978 A New Method for Assessing the Association Between Distributions of Points in Archaeology. In Simulation Studies in Archaeology. Ian Hodder, Ed. Cambridge University Press, Cambridge: 97-105.

Hodder, I. and C. Orton

1976 Spatial Analysis in Archaeology. Cambridge University Press, Cambridge.

Hodson, F. R.

1970 Cluster Analysis and Archaeology: Some New Developments and Applications. World Archaeology 1(3): 299-320.

Hoffecker, J.F.

2002 Desolate Landscapes: Ice-Age Settlement in Eastern Europe. Rutgers University Press, New Brunswick.

Johnson, I.

1984 Cell Frequency Recording and Analysis of Artefact Distributions. In Intrasite Spatial Analysis in Archaeology. Harold Hietala, Ed. Cambridge University Press, Cambridge: 75-96.

Keeley, L.H.

1991 Tool Use and Spatial Patterning: Complications and Solution. In The Interpretation of Archaeological Spatial Patterning. Ellen M. Kroll and T. Douglas Price, Eds. Plenum Press, New York. Pp.257-268. 
Kincaid, C.

1988 Predictive Modeling and its Relationship to Cultural Resource Management Applications. In Quantifying the Present and Predicting the Past: Theory, Method and Application of Archaeological Predictive Modeling. J. W. Judge and L. Sebastian, Eds. US Department of the Interior, Bureau of Land Management, Denver, Colorado: 549-569.

Kintigh, K. W.

1990 Intrasite Spatial Analysis: A Commentary on Major Methods. In Mathematics and Information Science in Archaeology. A. Voories, Ed: 165-200.

1994 Tools for Quantitative Archaeology: Programs for Quantitative Analysis in Archaeology. Available for Purchase at:

http://wwww.pages.prodigy.net/keith.kintigh

Kintigh, K. W. and A. J. Ammerman

1982 Heuristic Approaches to Spatial Analysis in Archaeology. American Antiquity 47(1): 31-63.

Knoerl, J. J.

1991 Mapping History Using Geographic Information Systems. The Public Historian 13(3): 97-107.

Koetje, T. A.

1989 Approaches to the Investigation of Intra-site Spatial Patterning in Three Dimensions. PhD Dissertation. University of Tennessee

1990 A Simulation Approach to Recovering Archaeologically Meaningful Levels. Journal of Quantitative Anthropology 2:257-280.

1991 Simulated Archaeological Levels and the Analysis of Le Flageolet II, the Dordogne, France. Journal of Field Archaeology 18:187-198.

1992 The Simulation and Recovery of Archaeologically Meaningful Levels. In Archaeology and the Information Age: A Global Perspective, P. Reilly and S. Rahtz, Eds. Routledge, London: 177-187.

1993 Site Formation Processes and Behavioural Deposition Episodes: The View from Le Flageolet II. In Formation Processes in Archaeological Context, P. Goldberg, D. T. Nash and M. D. Petraglia, Eds. Prehistoric Press, Madison, Wisconsin: 113-123.

1994 Intrasite Spatial Structure in the European Upper Palaeolithic: Evidence and Patterning from the SW of France. Journal of Anthropological Archaeology 13:161-169. 
Kroll, E. M. and T. D. Price

1991 The Interpretation of Archaeological Spatial Patterning. Interdisciplinary Contributions to Archaeology. Plenum Press, New York.

Kvamme, K. L.

1989 Geographic Information Systems in Regional Archaeological Research and Data Management. In Archaeological Method and Theory. M. B. Schiffe, Ed. Tucson, University of Arizona Press. 1: 139-203.

1993 Spatial Statistics and GIS: an Integrated Approach. In Computing the Past: Computer Applications and Quantitative Methods in Archaeology CAA92. I. Scollar, J. Andresen and T. Madsen, Eds. Aarhus, Aarhus University Press: 91-103.

1995 A View from Across the Water: the North American Experience in Archaeological GIS. In Archaeology and Geographical Information Systems: A European Perspective. Taylor and Francis, London: 1-13.

Kvamme, K. L. and T. A. Kohler 1988 Geographic Information Systems: Technical Aids for Data Collection, Analysis and Display. In Quantifying the Present and Predicting the Past: Theory, Method and Application of Archaeological Predictive Modeling. J. W. Judge and L. Sebastian, Eds. US Department of the Interior, Bureau of Land Management, Denver, Colorado: 493-548.

Limp, W. F.

2000 Geographic Information Systems in Historic Preservation. In Science and Technology in Historic Preservation. Williamson and Nickens. New York, Kluwer Academic/Plenum Publishers: 231-247.

Llobera, M.

1996 Exploring the Topography of Mind: GIS, Social Space and Archaeology. Antiquity 70: 612-622.

Lock, G. R. and T. M. Harris

1996 Danebury Revisited: An English Iron Age Hillfort in a Digital Landscape. In Anthropology, Space, and Geographic Information Systems. M. Aldenderfer and H. D. G. Maschner, Eds. Oxford University Press, New York: 214-240.

Marble, D. F.

1990 The Potential Methodological Impact of Geographic Information Systems on the Social Science. In Interpreting Space: GIS and Archaeology. K. M. S. Allen, S. W. Green and E. B. W. Zubrow, Eds. Taylor and Francis, London: 9-21. 
Marean, C. W. and L. Bertino

1994 Intrasite Spatial Analysis of Bone: Subtracting the Effect of Secondary

Carnivore Consumers. American Antiquity 59(4): 748-768.

Marozas, B. A.

1991 The Role of Geographic Information Systems in American Indian Land and

Water Rights Litigation. American Indian Culture and Research Journal 15(3): 77-93.

Marks, A. E. and V. P. Chabai

2001 Constructing Middle Palaeolithic Settlement Systems in Crimea:

Potentials and Limitations. In Settlement Dynamics of the Middle Palaeolithic and Middle Stone Age. Nicholas J. Conard, Ed. Pp 179-201.

1998 The Middle Palaeolithic of Western Crimea, Vol. 1 of The Palaeolithic of Crimea, I. Liege: ERAUL 84.

Maschner, H. D. G.

1996a Theory, Technology and the Future of Geographic Information Systems in Archaeology. In New Methods, Old Problems: GIS in Modern Archaeological Research, H. D. G. Maschner, Center for Archaeological Investigations, Southern Illinois University at Carbondale, Illinois: 301-307.

1996b Geographic Information Systems in Archaeology. New Methods, Old Problems. In Geographic Information Systems in Modern Archaeological Research. H. D. G. Maschner. Ed. Center for Archaeological Investigations, Illinois, Occasional Paper No. 23: 1-21.

McKelway, H. S., P. J. Carr and T. Koetje 1992 A New Approach for Reconstructing and Interpreting Artifact Context in Historic Features. In Proceedings of the Tenth Symposium on Ohio Valley Urban and Historic Archaeology, A. L. Young and C. H. Faulkner, Eds. Miscellaneous Paper no. 16. Tennessee Anthropological Association, Tennesse.

McGrew, J.C. Jr. and C.B. Monroe 2000 An Introduction to Statistical Problem Solving in Geography. McGraw Hill, Boston.

McNutt, C. H.

1981 Nearest Neighbours, Boundary Effect and the Old Flag Trick: A General Solution. American Antiquity 46(3): 571-592.

Mellars, $\mathbf{P}$.

1995 The Neanderthal Legacy: An Archaeological Perspective from Western Europe. Princeton University Press, Princeton. 
Orton, C. R.

1982 Stochastic Process and Archaeological Mechanism in Spatial Analysis.

Journal of Archaeological Science 9: 1-23.

1996 Mathematics in Archaeology. Cambridge University Press, Cambridge.

Pettitt, P. B.

1997 High Resolution Neanderthals? Interpreting Middle Palaeolithic Intrasite

Spatial Data. World Archaeology 29(2): 208-224.

Pinder, D., I. Simada and D. Gregory

1979 The Nearest Neighbour Statistic: Archaeological Application and New

Developments. American Antiquity 44(3): 430-445.

Rick, J. W.

1976 Downslope Movement and Archaeological Intrasite Spatial Analysis. American Antiquity 41(2): 133-144.

Rigaud, J.P. and J. F. Simek

1991 Interpreting Spatial Patterns at the Grotte XV - A Multiple-Method Approach. In The Interpretation of Archaeological Spatial Patterning, E. M. Kroll and T. D. Price, Eds. Plenum Press, New York: 199-220.

Rowlett, R. M. and M. C. Robbins

1982 Estimating Original Assemblage Content to Adjust for Post-Depositional Vertical Artifact Movement. World Archaeology 14(1): 73-83.

Ruggles, A. J. and R. L. Church

1996 Spatial Allocation in Archaeology: An Opportunity for Reevaluation. In New Methods, Old Problems: GIS in Modern Archaeological Research, H. D. G. Maschner, Ed. Center for Archaeological Investigations, Southern Illinois University at Carbondale, Illinois: 147-173.

Savage, S. H.

1990 GIS in Archaeological Research. In Interpreting Space: GIS and Archaeology. K. M. S. Allen, S. W. Green and E. B. W. Zubrow, Eds. Taylor and Francis, London: 22-31.

Schiffer, M. B.

1972 Archaeological Context and Systemic Context. American Antiquity 37(2): 156-165.

Simek, J. F.

1984a Integrating Pattern and Context in Spatial Archaeology. Journal of Archaeological Science 11:405-420. 
1984b A K-Means Approach to the Analysis of Spatial Structure in Upper

Palaeolithic Habitation Sites. BAR International Series 205, Oxford, England.

1987 Spatial Order and Behavioural Change in the French Palaeolithic. Antiquity 61: 25-40.

Simek, J. F., A. J. Ammerman and K. W. Kintigh 1985 Explorations in Heuristic Spatial Analysis: Analyzing the Structure of Material Accumulation Over Space. In To Pattern the Past. A. Voorrips and S.H. Loving, Eds. PACT II, Strasbourg: 229-247.

Simek, J. F. and R. R. Larick 1983 The Recognition of Multiple Spatial Patterns: a Case Study from the French Upper Palaeolithic. Journal of Archaeological Science 10:165-180.

Speth, J. D. and G. A. Johnson 1976 Problems in the Use of Correlation for the Investigation of Tool Kits and Activity Areas. In Cultural Change and Continuity: Essays in Honour of James Bennett Griffin, C.E. Cleland, Ed. Academic Press, New York: 35-57.

Spikins, P., C. Conneller, H. Ayestaran and B. Scaife 2002 GIS Based Interpolation Applied to Distinguishing Occupation Phases of Early Prehistoric Sites. Journal of Archaeological Science 29:1235-1245.

SPSS

1999 SPSS Base 10.0 Applications Guide. SPSS, Inc., Chicago, Illinois.

Spurling, B. and B. Hayden

1984 Ethnoarchaeology and Intrasite Spatial Analysis: A Case Study from the Australian Western Desert. In Intrasite Spatial Analysis in Archaeology, $\mathrm{H}$. Hietala, Ed. Cambridge University Press, Cambridge: 224-241.

Stapart, D.

1989 The Ring and Sector Method: Intrasite Spatial Analysis of Stone Age Sites, with Special Reference to Pincevent. Paleohistoria 31: 1-57.

1990 Middle Palaeolithic Dwellings: Fact or Fiction? Some Applications of the Ring and Sector Method. Paleohistoria 32: 1-20.

Stapart, D. and M. Street 1997 High Resolution or Optimum Resolution? Spatial Analysis of the Federmesser site at Andernach, Germany. World Archaeology 29(2): 172-194.

Stepanchuk, V. N. 1993 Prolom II, a Middle Palaeolithic Cave Site in the Eastern Crimea with Non-Utilitarian Bone Artefacts. Proceedings of the Prehistoric Society 59:17-37. 
Stine, R.S. and D.P. Lanter

1990 Considerations for Archaeology Database Design. In Interpreting Space: GIS and Archaeology. K.M.S Allen, S.W. Green and E.B.W. Zubrow, Eds. Taylor and Francis, London: 80-89.

Straus, L. G.

1979 Caves: a Palaeoanthropological Resource. World Archaeology 10(3): 331339.

Thomas, D. H.

1986 Refiguring Anthropology: First Principles of Probability and Statistics. Waveland Press, Inc., Illinois.

van Leusen, P. M.

1995 GIS and Archaeological Resource Management: a European Agenda. In Archaeology and Geographical Information Systems: A European Perspective. G.

R. Lock and Z. Stancic, Eds. Taylor and Francis, London: 27-41.

Vaquero, $\mathrm{M}$.

1999 Intrasite Spatial Organization of Lithic Production in the Middle Palaeolithic: the Evidence of the Abric Romani (Capellades, Spain). Antiquity 73: 493-504.

Vaquero, M. and I. Pasto 2001 The Definition of Spatial Units in Middle Palaeolithic Sites: The HearthRelated Assemblages. Journal of Archaeological Science 28:1209-1220.

Vierra, R.K. and R.L. Taylor 1977 Dummy Data Distribution and Quantitative Methods: An Example Applied to Overlapping Spatial Distributions. In For Theory Building in Archaeology: Essays on Faunal Remains, Aquatic Resources, Spatial Analysis and Systemic Modeling. Lewis Binford, Ed. Academic Press, New York: 317-326.

Villa, P.

1982 Conjoinable Pieces and Site Formation Processes. American Antiquity 47(2): $276-290$.

Villa, P. and J. Courtin

1983 The Interpretation of Stratified Sites: A View from Underground. Journal of Archaeological Science 10: 267-281.

Wandsnider, L. 1996 Describing and Comparing Archaeological Spatial Structures. Journal of Archaeological Method and Theory 3(4): 319-383. 
Wansleeben, $\mathrm{M}$.

1988 Applications of Geographical Information Systems in Archaeological Research.In Computer Applications and Quantitative Methods in Archaeology 1988. S. P. Q. Rahtz, Ed. British Archaeology Reports, Oxford: 435-451.

Warren, R.E.

1990 Predictive Modelling of Archaeological Site Location: A Case Study in The Midwest. In Interpreting Space: GIS and Archaeology. K.M.S. Allen, S.W. Green and E.B.W. Zubrow, Eds. Taylor and Francis, London: 201-215.

Whallon, R., Jr.

1973 Spatial Analysis of Occupation Floors I: Application of Dimensional Analysis of Variance. American Antiquity 38(3): 266-277.

1974 Spatial Analysis of Occupation Floors II: The Application of Nearest Neighbour Analysis. American Antiquity 39(1): 16-34.

Wheatly, D. and M. Gillings 2002 Spatial Technology and Archaeology: The Archaeological Application of GIS. Taylor and Francis, London.

Yevtushenko, A.I. and A. Burke In Press The Exploitation of Highland Regions in the Crimean Middle Palaeolithic: Preliminary Evidence from Karabi Tamchin.

Yevtushenko, A.I., A. Burke, C.R. Ferring, V. Chabai, K. Monigal In Press a The Middle Palaeolithic Site of Karabi Tamchin (Crimea, Ukraine): 1999-2001 Excavation Seasons. Papers of the Prehistoric Society. (Dec. 2003)

Yevtushenko, A.I., A. Burke, C.R. Ferring In Press $b$ The Site of Karabi Tamchin: Introduction.

Zubrow, E. B. W. 1990 Modelling and Prediction with Geographic Information Systems: A Demographic Example from Prehistoric and Historic New York. In Interpreting space: GIS and Archaeology. K. M. S. Allen, S. W. Green and E. B. W. Zubrow, Eds. Taylor and Francis, London: 307-318.

Zulick, C. A.

1986 Application of a Geographic Information System to the Bureau of Land Management's Resource Management Planning Process. In Geographic Information Systems in Government Vol. 1. B. K. Opitz, Ed. A. Deepak Publishing, Hampton, Virginia: 309-328. 